\begin{tabular}{|l|c|r|}
\hline & Canberra Industries, ACK\# D0043 & 33296B.DOC \\
\hline Created: $29-$ September-2000 & IBIS Project & Revision B \\
\hline Revised: 13-January-2003 & Preliminary Design Study & \\
\hline
\end{tabular}

\title{
Integrated Box Interrogation System (IBIS) Preliminary Design Study
}

\author{
Prepared For \\ U.S. Department of Energy \\ Boxed Waste Form Nondestructive Assay \\ Development \& Demonstration \\ (DE-FC07-00ID13812)
}

Originator: Alephen Cott. Date: $\frac{01 / 13 / 03}{01 / 13 / 03}$ 


\begin{tabular}{|l|c|r|}
\hline & Canberra Industries, ACK\# D0043 & 33296 B \\
\hline Created: 29-September-2000 & IBIS Project & Revision B \\
\hline Revised: 13-January-2003 & Preliminary Design Study & \\
\hline
\end{tabular}

\section{'IBIS' - THE INTEGRATED BOX INTERROGATION SYSTEM}

Dr Stephen Croft, Mr. David Martancik and Dr Brian Young Canberra Industries, 800 Research Parkway, Meriden CT, 06450, USA

Dr Patrick MJ Chard

Harwell Instruments Ltd.

528.10 Unit 1 , Harwell International Business Centre, Didcot, Oxfordshire OX11 0RA

Dr Robert J Estep, Sheila Melton and Gaetano J Arnone Advanced Nuclear Technology Group (NIS-6)

MS-J562, Los Alamos National Laboratory, Los Alamos, NM 87545, USA 


\begin{tabular}{|l|c|r|}
\hline & Canberra Industries, ACK\# D0043 & 33296 B \\
\hline Created: 29-September-2000 & IBIS Project & Revision B \\
\hline Revised: 13-January-2003 & Preliminary Design Study & \\
\hline
\end{tabular}




\begin{tabular}{|c|c|c|c|}
\hline \multirow{3}{*}{ 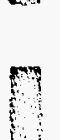 } & & Canberra Industries, ACK\# D0043 & $33296 \mathrm{~B}$ \\
\hline & Created: 29-September-2000 & IBIS Project & Revision B \\
\hline & Revised: 13-January-2003 & Preliminary Design Study & Page i of 156 \\
\hline
\end{tabular}

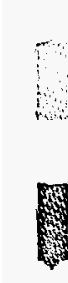

P

\section{Initial Revision ECN}

$\mathrm{SC} \quad \mathrm{A}$

$\mathrm{SC} \quad \mathrm{B}$

\section{$\underline{\text { Revision History }}$}

Date Comment

29-September-2000 Initial Release, Issued to customer for comment

13-January-2003 Minor clean-up 


\begin{tabular}{|l|r|r|}
\hline & Canberra Industries, ACK\# D0043 & 33296 B \\
\hline Created: 29-September-2000 & IBIS Project & Revision B \\
\hline Revised: 13-January-2003 & Preliminary Design Study & Page ii of 156 \\
\hline
\end{tabular}

\section{Table of Contents}

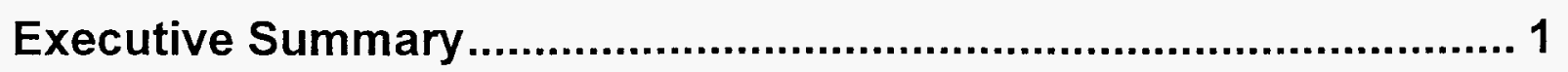

IBIS

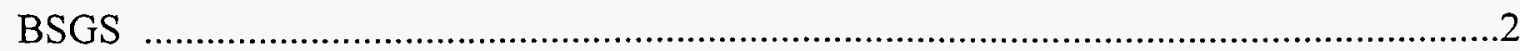

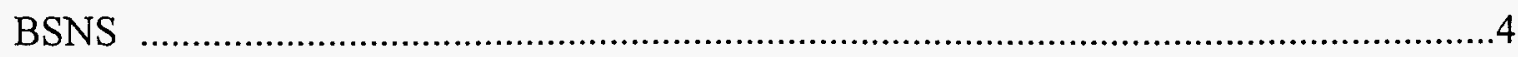

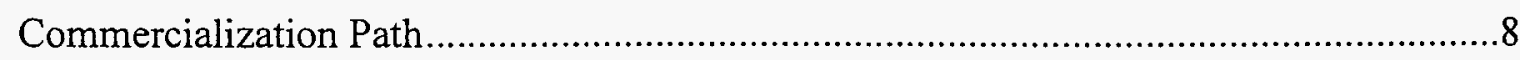

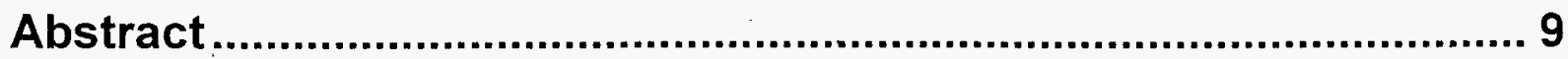

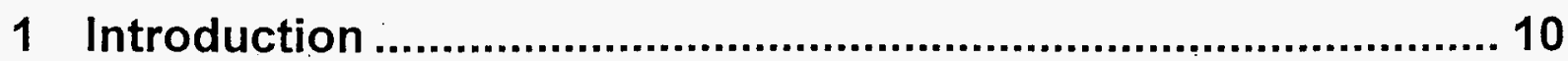

2 Program Structure .............................................................. 12

3 The System ......................................................................... 15

3.1 Characterization and Correction for Matrix Heterogeneity ..................................17

3.2 Characterization of Non-Uniform Radio-Nuclide and Isotopic Compositions ......17

3.3 Assay of Difficult Matrices .........................................................................18

4 Box Segmented Gamma-Ray Scanner..................................... 19

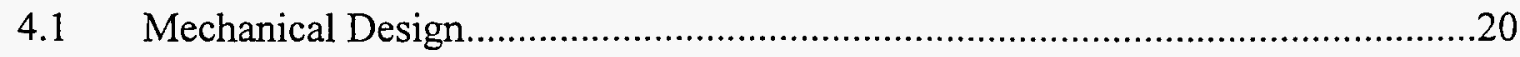

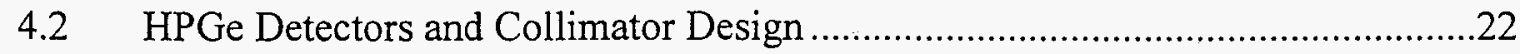

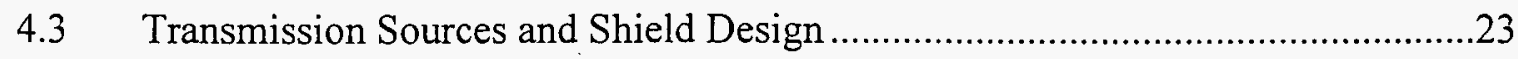

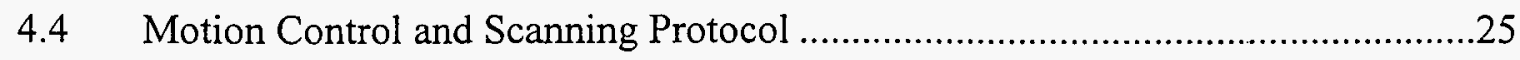

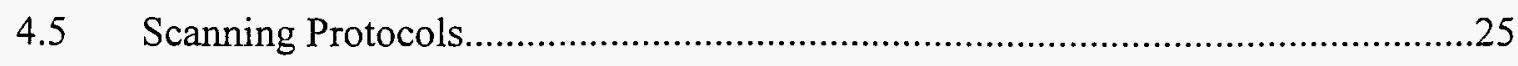

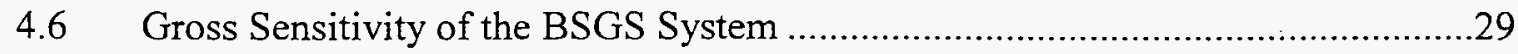

4.7 The Problems of Source and Matrix Non-uniformity …........................................

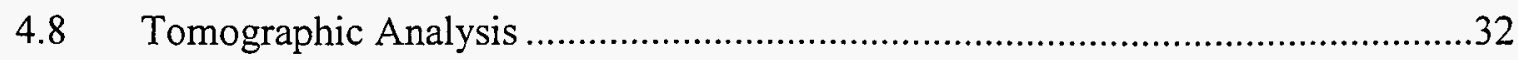

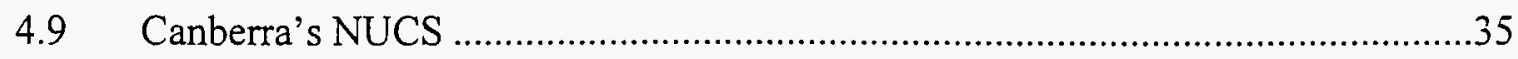

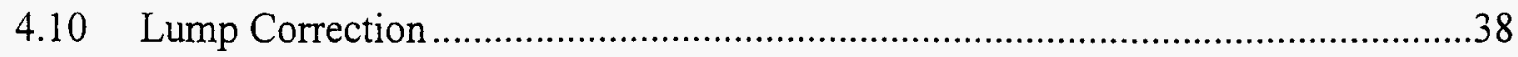

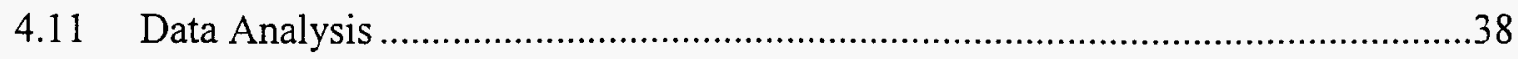

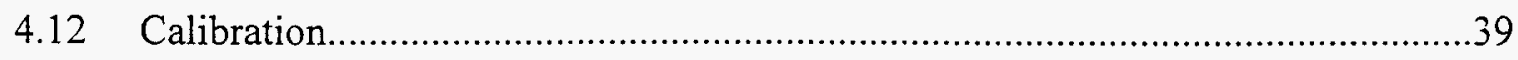

5 Differential Die-Away Analysis ............................................. 41

6 Box Segmented Neutron Scanner............................................ 47

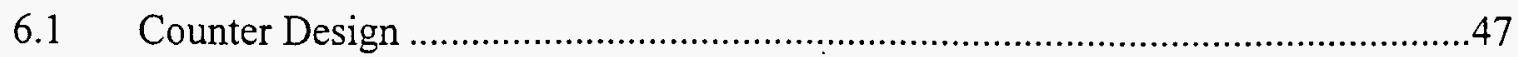

6.2 List Mode Data Acquisition............................................................................47 


\begin{tabular}{|l|c|r|}
\hline & Canberra Industries, ACK\# D0043 & 33296 B \\
\hline Created: 29-September-2000 & IBIS Project & Revision B \\
\hline Revised: 13-January-2003 & Preliminary Design Study & Page iii of 156 \\
\hline
\end{tabular}

6.3 Advanced Passive Neutron Assay Analysis ....................................................53

6.4 Integration of the Add-A-Source (AAS) Correction Technique ...........................54

6.5 Physical Form of Radioactive Material............................................................54

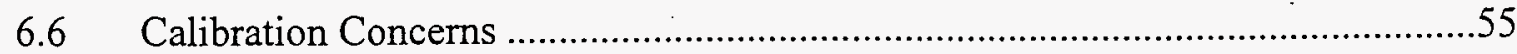

7 Design Study for the IBIS Neutron Counting System ................ 56

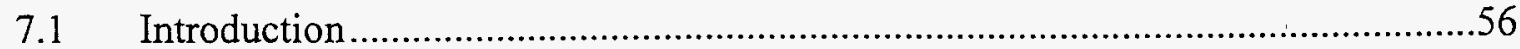

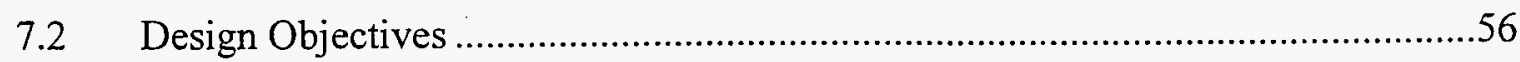

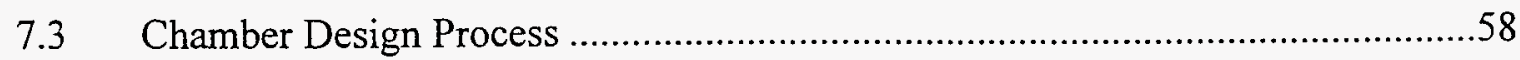

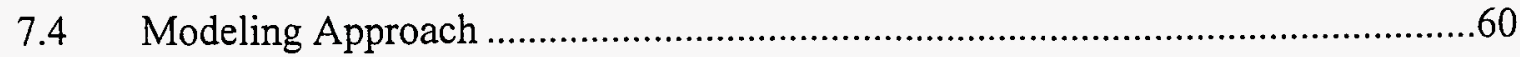

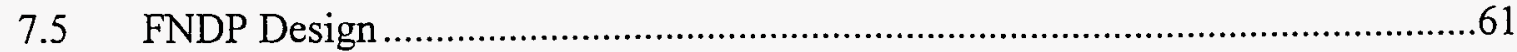

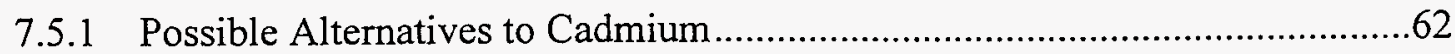

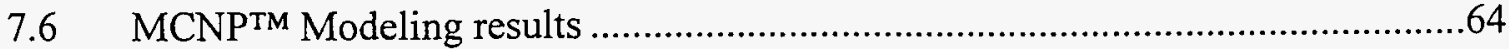

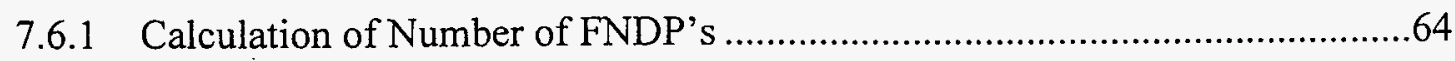

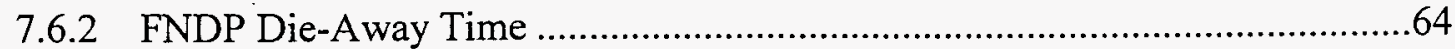

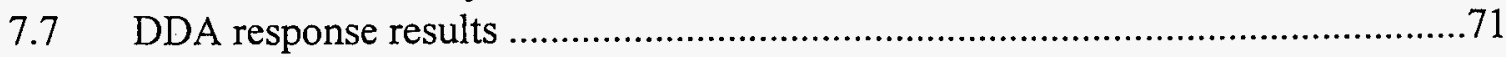

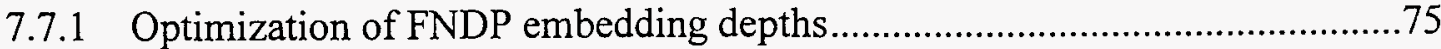

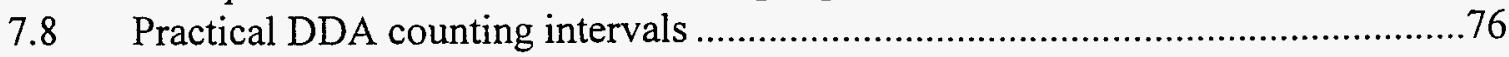

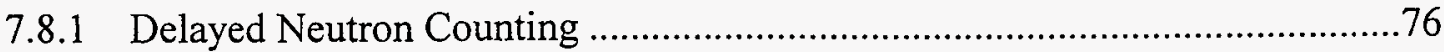

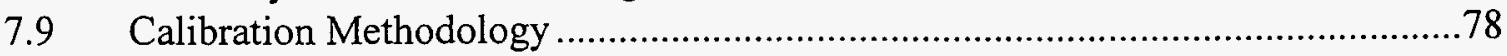

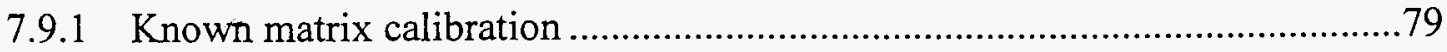

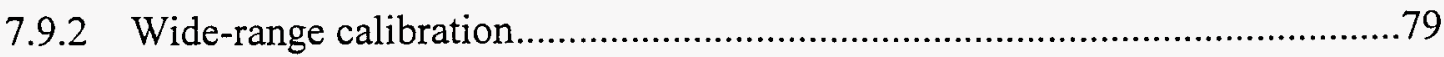

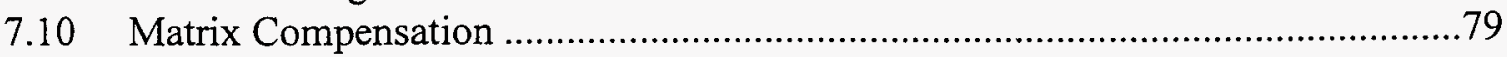

7.11 Experimental Experience with Matrix Monitor Resolution .............................82

8 Brief Note on LANL MCNP TM Design Calculations .....................8 86

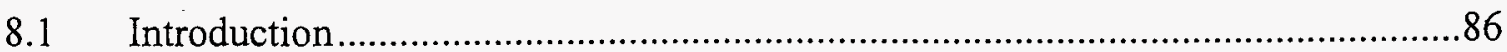

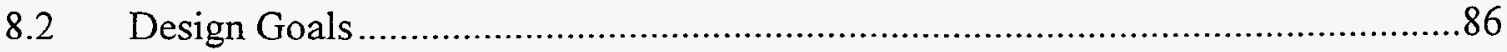

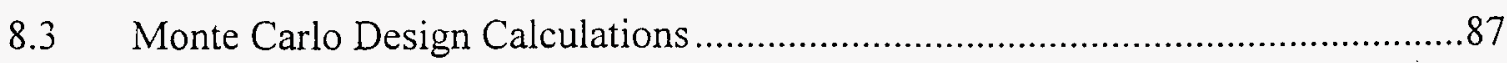

8.3.1 Benchmark Model with CWAM Calibration Data ........................................88

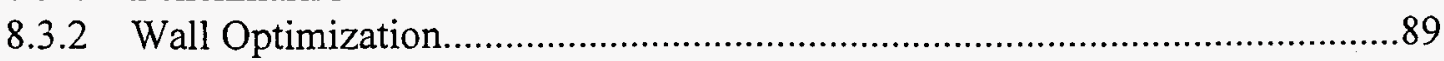

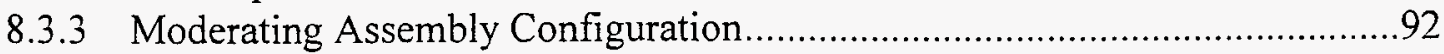

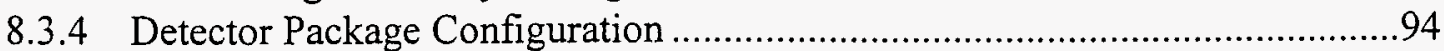

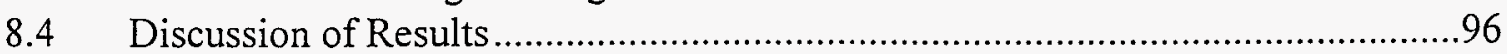

9 Self Shielding in Lumps of Fissile Material............................. 98

10 Integration of Neutron and Gamma-Ray Results ................... 103

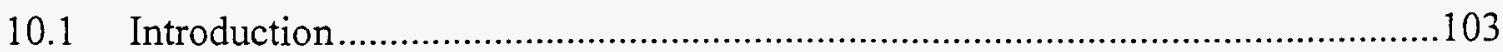

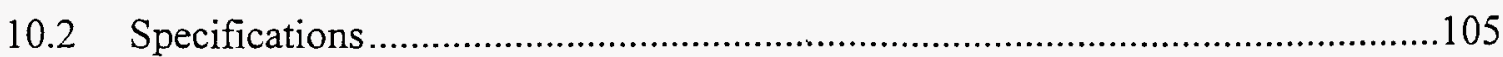




\begin{tabular}{|l|c|r|}
\hline & Canberra Industries, ACK\# D0043 & 33296 B \\
\hline Created: 29-September-2000 & IBIS Project & Revision B \\
\hline Revised: 13-January-2003 & Preliminary Design Study & Page iv of 156 \\
\hline
\end{tabular}

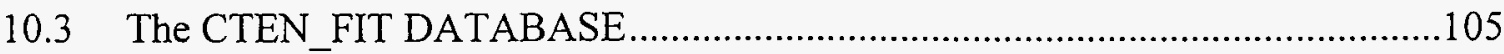

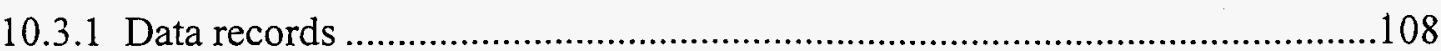

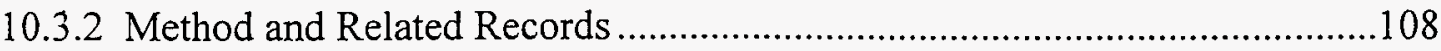

10.3.3 Results Records and the Analysis Queue..................................................111

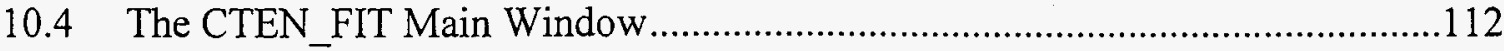

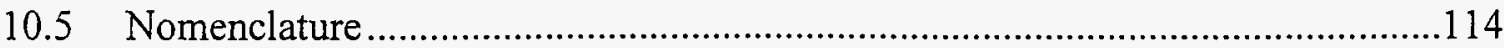

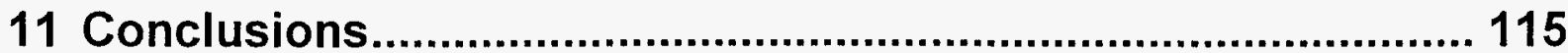

\section{A. First Principles Derivation of the FNDP Intrinsic Decay Time} Constant .................................................................................. 117

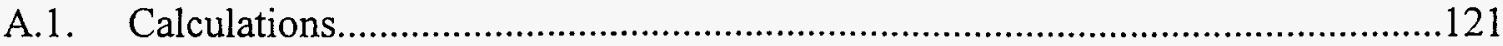

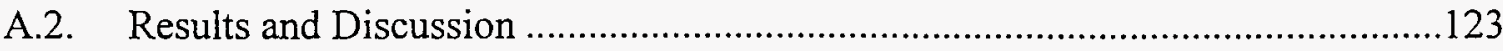

B. Measured Response of the CWAM System ............................ 126

B.1 Active Measurements the in CWAM System ................................................134

B.2 Sensitivity for the Active Assay............................................................136

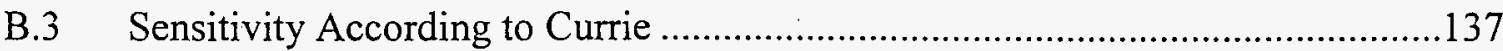

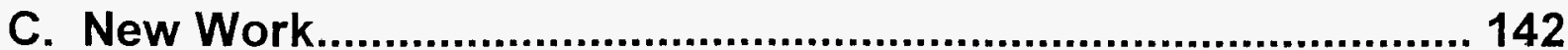

D. References and Bibliography ................................................ 144 


\begin{tabular}{|l|l|r|}
\hline & Canberra Industries, ACK\# D0043 & 33296 B \\
\hline Created: 29-September-2000 & IBIS Project & Revision B \\
\hline Revised: 13-January-2003 & Preliminary Design Study & Page 1 of 156 \\
\hline
\end{tabular}

\section{Executive Summary}

\section{IBIS}

This report outlines the conceptual design studies performed in support the Integrated Box Interrogation System, IBIS. IBIS is comprises two separate measurement stations delivering complementary data that is integrated by software. An emphasis on acquiring multiple distinct geometrical views of the waste using multiple complimentary techniques has resulted in a instrument design that offers the potential of recording the most comprehensive set of assay data of any box counter to date. This permits tomographic matrix corrections and (active) image spatial corrections to be extracted. Consequently it is expected that the integration software will produce more accurate assays and allow measurements over a larger dynamic range while at the same time being less dependent on a priori assumptions about the waste. This means that more boxes are amenable to direct assay. Although it is difficult to make accurate predictions we estimate that current nondestructive analysis methods are capable of measuring only about $30 \%$ of the DOE's boxed waste inventory without indeterminate error (may be as large as several hundred percent due to the five major issues). The research and development efforts proposed here are focused on advancing identification and correction methods that address sources of measurement bias. These advances may allow the IBIS to be able to directly assay, to acceptable sensitivity and accuracy to meet regulatory compliance, perhaps as much as $60 \%$ to $80 \%$ of the boxed and crated waste inventory. This represents a factor of over two increase compared against existing technologies. Furthermore the call for expert data review, intrusive intervention and other special needs such as interim storage will be reduced. Some $95 \%$ of waste will be packaged in containers measuring 5' x 5' x 8' or less (Dave Moody CBFO Feb 2001 PrivComm).

The IBIS is intended to assay TRU and Low Level boxed waste to an accuracy and precision suitable for WIPP disposal and to satisfy operational requirements, transport, criticality and radionuclide reporting. A recent review of technologies available for the non-destructive assay of TRU and LL boxed waste forms identified a clear gap between what is currently available and what is needed to measure both the large volume of waste presently in interim storage and the large volume of future arisings expected to be generated as a result of site clean-ups and closure. IBIS is an attempt to address these weaknesses.

IBIS is comprised of a relocatable standalone Box Segmented Gamma Scanner (BSGS), and a the trailer mounted Box Segmented Neutron Scanner (BSNS). The performance of the BSGS has been scoped using the Canberra ISOCS code and that of the BSNS has been assessed using the LANL radiation transport code MCNPTM .

The report is organized as follows. Chapters 1 and 2 introduce the objective of the project and outlined the project structure. Chapter 3 provides a top level overview of the system. In chapter 4 , the BSGS is discussed in detail. The detector arrangement and performance capability are presented. A major component of the IBIS is the differential die-away analysis capability. Chapter 5 reviews the origin of this method, and indicates why we feel it is an attractive technique around which to assembly a next generation boxed waste counter. The top level 


\begin{tabular}{|l|l|r|}
\hline & Canberra Industries, ACK\# D0043 & 33296 B \\
\hline Created: 29-September-2000 & IBIS Project & Revision B \\
\hline Revised: 13-January-2003 & Preliminary Design Study & Page 2 of 156 \\
\hline
\end{tabular}

functional description of the BSNS is presented in Chapter 6. This sections contains a detailed discussion of the advantages of using list mode data acquisition for this application and introduces some of the novel data analysis options that will be available. Chapters 7 and 8 encapsulate the technically detailed neutronic design studies performed during Phase 1 of the project. Chapter 7 concentrates on generic and theoretical aspects (i.e. the guiding principles), and covers novel ideas (such as the potential to use Gd instead of $\mathrm{Cd}$ as the shield around the fast neutron detector packages) and the practical limits to spatial resolution study. Appendix $A$ is associated with chapter 7 and gives a pedagogic treatment of the key factors affecting design of the fast neutron detector package. Chapter 8 summarizes more detailed and specific design optimization MCNPTM simulations and is intended to establish actual build parameters such as wall structures and moderator assembly block materials and dimensions. Performance parameters are traceable to actual experimental data taken with CWAM. The CWAM data is outlined in Appendix B. The self shielding affect is discussed and quantified in chapter 9 . This is the reason why the differential die-away method may under report the mass of fissile material when lumps of material are present. The software integration of the neutron and gamma ray results is treated in chapter 10. This represents a major part of the development challenge. Finally, in chapter 11 a brief concluding remark is made reaffirming the authors' belief that the IBIS is a significant advance in the technology available for assaying boxed waste. The system proposed offers numerous advantages over previously designed crate counting systems, and would undoubtedly stretch the current state-of-the-art.

The salient conclusions of this study

\section{BSGS}

- The gamma-ray system will provide Pu and U isotopic weight fraction information and quantitative assay data for these elements to support the neutron assay system in some circumstances. It provides the primary means of quantifying activation and fission product activities that is in the RNI (RadioNuclide inventory) information.

- The hardware associated with the BSGS is considered to be conventional and low risk. The novel feature lies in the flexibility of the data taking available from this design. This allows a comprehensive array of analysis options to be considered.

- The design uses modular components to assist fast disassembly and re-assembly elsewhere.

- By placing detectors not only on the sides but also above and below the box additional spatial information and analysis options become available compared to other gamma box counters. This arrangement directly addresses accuracy problems that arise from highly heterogeneous and dense matrix material and non-uniform distributions of gamma emitter.

- The performance of the BSGS can be simulated fairly accurately using existing gamma-ray transport codes such as MCNP ${ }^{\mathrm{TM}}$ and ISOCS. For deep gamma penetration problems, the 


\begin{tabular}{|l|l|r|}
\hline & Canberra Industries, ACK\# D0043 & 33296 B \\
\hline Created: 29-September-2000 & IBIS Project & Revision B \\
\hline Revised: 13-January-2003 & Preliminary Design Study & Page 3 of 156 \\
\hline
\end{tabular}

treatment of multiple small angle scattering and of coherent scattering has been identified as an area where improvements can be made in the way modeling has conventionally been done.

- The BSGS suffers severe under reporting of fissile mass when self attenuation caused by gram sized lumps is present. There is no practical way of accurately correcting for this. Even in favorable cases the correction methodologies proposed to date fail when needed the most. Quantification of this statement to amplify the underlying physical reasons would require a desk study. The traditional approach of calibrating the system for realistic conditions and factoring into the TMU an allowance based on a knowledge of the waste stream generation process remains the only broadly applicable approach for the NDA of existing large packages.

- Numerical simulations are recommended as the best way to test the benefit of several new proposed data analysis methods over an extended matrix set. This can be more flexible, faster and less expensive than performing measurements on actual surrogate matrices. The matrices used in the measurement program to benchmark the gamma-ray simulations will be those required for establishing the neutron calibration. The neutron response is more sensitive to detailed elemental composition than is the gamma-ray response.

- Matrix corrections are severely limited, especially at low energies (e.g. the $186 \mathrm{keV}$ line of ${ }^{235} \mathrm{U}$ and the 129,204 and $345 \mathrm{keV}$ lines of ${ }^{239} \mathrm{Pu}$ ), for the larger boxes due to the very low transmission factors of soft gamma-rays. Thus simplifying assumptions related to uniformity and homogeneity are inevitable. It is proposed to map the matrix density along the length of the drum using horizontally and vertically aligned ${ }^{60}$ Co transmission sources. Short of rotating the box, which is not considered practical, this arrangement gives the most spatial information possible on which to build data driven gamma-ray matrix correction factors. HRGS allows the use of other transmission sources such as mixed ${ }^{133} \mathrm{Ba},{ }^{137} \mathrm{Cs}$ and ${ }^{60} \mathrm{Co}$ or multi-line ${ }^{152} \mathrm{EU}$. However for the larger denser boxes the low energies are severely attenuated so that it makes sense to concentrate the count rate capability on the penetrating lines from ${ }^{60} \mathrm{Co}$ to derive essentially a density map.

- The visibility of fissile material in the cental regions of the larger densely filled boxes will be very poor. The ability to derive activity maps by the differential gamma attenuation method is limited to an outer skin of the order of $300 \mathrm{~mm}$ and will be degraded by the presence of other gamma emitters that contribute to the background. Therefore the possibility of serious assay inaccuracy can not in general be ruled out using correction factors derived from the gammaray data alone and the TMU estimate remains largely subjective relying on worst case assumptions. These place a high upper bound on the mass estimate. Integration of the positional dependent information from the BSGS with that of the BSNS will be explored as a way of reducing the magnitude of this potential error. 


\begin{tabular}{|l|l|r|}
\hline & Canberra Industries, ACK\# D0043 & 33296 B \\
\hline Created: 29-September-2000 & IBIS Project & Revision B \\
\hline Revised: 13-January-2003 & Preliminary Design Study & Page 4 of 156 \\
\hline
\end{tabular}

\section{BSNS}

- The approach is to use high performance differential die-away measurements to rapidly and accurately screen LLW and dilute distributions of Pu and to rely more on the passive neutron coincidence counting technique for the higher mass loadings of Pu present in TRU waste. The active assay also provides quantification of $\mathrm{U}$, about which passive neutron counting offers no information, and also confers immunity to (high alpha-n) rates and the presence of $\mathrm{Cm}$ which can degrade the performance of purely passive neutron based assay systems for TRU. Because of this we consider that achieving a high performance DDA sub-system in combination with a high accuracy passive assay sub-system is more important than compromising the overall performance in a drive simply to achieve the highest passive sensitivity. On these grounds we have selected a 4 detection arrangement with all of the efficiency being provided by $\mathrm{Cd}$ wrapped fast neutron detector packages. This provides a nearly isotropic and uniform detection efficiency within the empty cavity and non-interfering matrices. Furthermore, the $\mathrm{Cd}$ de-couples the detectors from certain matrix perturbations. In particular the dependence of the coincidence gate utilization factor on the matrix is much reduced, and the importance of thermal neutron (the abundance of which is strongly influenced by the moderator/absorber properties of the matrix), are strongly suppressed compared to the situation where bare ${ }^{3} \mathrm{He}$ tubes are deployed. An additional benefit is that the ambient Totals background rate from waste in storage or transit nearby is reduced. However, in using exclusively $\mathrm{Cd}$ wrapped detectors a balance must be struck between achieving a high efficiency and achieving the desired high specific active response. The active response is proportional to the product of the detection efficiency and the interrogating neutron flux in active prompt Totals mode and the efficiency squared flux product in active coincidence mode. The need to carefully balance the design arises due to the fact that as the efficiency is increased the interrogating flux is poisoned. For trailer mounted designs, such as called for here, weight and size limitations prohibit the embedding of the fast neutron detector packages deep within a thick graphite wall which is the usual way of removing the poison from the regions of importance for the generation and maintenance of the thermal flux. Our original design intent was to design a system with an efficiency of $14 \%$ to at least match the performance of the CWAM system. By using higher grade graphite and by thoughtful design, including a modest moderating assembly around the neutron generator, we are confident that we can fabricate a system with an efficiency of circa $17 \%$ using 24 fast neutron detector packages, each containing four 2 inch diameter ${ }^{3} \mathrm{He}$ tubes, within a more compact and light weight wall design commensurate with trailer mounting.

- Doubling the number of fast neutron detectors has a major impact on cost but only a relatively minor impact on extending the performance envelope of the passive neutron subsystem into the lower Pu mass range which is differential die-away techniques forte. Conversely, increasing the efficiency confers no benefit to the active sub-system because of the extra flux poisoning. Because of this we do not recommend increasing the efficiency within this work program above that currently projected. The projected efficiency of $17 \%$ is considered adequate to demonstrate all of the novel concepts, such as imaging of 


\begin{tabular}{|l|l|r|}
\hline & Canberra Industries, ACK\# D0043 & 33296 B \\
\hline Created: 29-September-2000 & IBIS Project & Revision B \\
\hline Revised: 13-January-2003 & Preliminary Design Study & Page 5 of 156 \\
\hline
\end{tabular}

concentrations of fissile material, that are to be investigated in this program and that will confer measurement advantage in the field.

- The hardware implementation is challenging but fundamentally can be considered to be only a modest risk as proven components and designs exist for all of the key components. The present work needs some refinement to meet the design goal of a trailer mounted unit. In particular the wall structure needs fine tuning to obtain the desired performance target within the DOT weight restriction.

- The PATRM/PCI board is discussed in some detail. It is a simple, compact and flexible list mode data taking device well suited for R\&D needs and for use in a mobile system. It forms the hub of both the active neutron and passive neutron counting assays. Much of the active and passive imaging capability, in both Totals and Coincidence modes, depends on the analysis of the list mode data the PATRM/PCI unit provides. Multiplicity analysis is available for data filtering and the unit allows certain performance and quality checks to be performed conveniently.

- The passive mode operation and performance can be modeled quite accurately using established techniques based on MCNP ${ }^{\mathrm{TM}}$ simulations and point model equations. Several large container counters have been built and tested before and these can be used as benchmarks.

- The largest errors (factors of several or more) in conventional neutron assays are the result of source positioning effects. The magnitude of these effects can be substantially reduced (almost eliminated) if good positional information is available. Passive and active singles imaging techniques are in their infancy. Scoping experiments are planned on small scale rigs during this project. This will help develop our thinking on how best to implement imaging techniques on IBIS. The spatial resolution achievable (of the order of $1 \mathrm{ft}$ ) is governed by physical principles. This is still sufficient to allow significant performance advantage to be obtained in a box counter geometry from the gamma system. The active assay has good sensitivity for fissile material. If the measured fissile material distribution (only the shape not the absolute level is needed) can be taken as representative of the $\mathrm{Pu}$ distribution, then the active image can serve as a spatial correction template for the passive (coincidence) assay, even at masses when forming an image by passive counting would be unviable due to poor counting precision. Similarly, the active image could be used to correct the gamma-ray assay for $\mathrm{U}$ and $\mathrm{Pu}$. Because of its high sensitivity the active system can see concentrations of material deep within a box at levels that would be below the minimum detectable quantity for gamma-ray assay. Furthermore the matrix effects (high density, high degree of moderation) ought to add contrast to the multiple views of the source and permit such cases to continue to be located with accuracy commensurate to the need. Previous attempts to incorporate neutron imaging have been made. For example the APNEA system associated with Dave Hensley uses the passive neutron data to form an image to help interpret the DDA results. At ORNL waste containing ${ }^{244} \mathrm{Cm}$ and ${ }^{252} \mathrm{Cf}$ sometimes is favorable to this approach even with a 


\begin{tabular}{|l|l|r|}
\hline & Canberra Industries, ACK\# D0043 & 33296 B \\
\hline Created: 29-September-2000 & IBIS Project & Revision B \\
\hline Revised: 13-January-2003 & Preliminary Design Study & Page 6 of 156 \\
\hline
\end{tabular}

modest $(13 \%)$ detection efficiency. The approach advocated here is to use a more sensitive DDA subsystem and to exploit the imaging potential of it directly.

- Passive and Active coincidence counting imaging is a novel idea but requires a more detailed study before definitive statements as to its feasibility can be made. The counting rates are likely to be quite low except when large masses are present. Large masses usually mean that there is a distributed source (in which case the accuracy improvement will be modest or that there is a lump problem (which in the case of the active mode means that there could be a serious under reporting problem).

- The DDA technique offers excellent sensitivity for screening land fill and LLW. The response is specific to fissile material. The assay time is much shorter than either the passive neutron or the passive gamma-ray techniques. The differential die-away method is insensitive to the two most common interferences that perturb passive neutron measurements, namely: the presence of $\mathrm{Cm}$ which mimics the presence of fertile $\mathrm{Pu}$ isotopes and the presence of high (alpha-n) emissions which degrade counting precision.

- By exploiting the principles of: 1) an 'undermoderated' assay cavity so that the interrogating thermal flux is self-generated within the matrix and if needed 2) the asymmetric detection efficiency geometry, the spatial variability of the active assay (flux - efficiency product) can be made much lower than is widely appreciated, even in difficult to measure cases. In addition, the enhanced flux in moderating matrices offsets the lost efficiency in the Cd covered fast neutron detector packages. This confers good accuracy in addition to good sensitivity in situations where self-shielding is not a problem.

- In active mode the DDA method suffers for under reporting of fissile lumps. There is no practical experimental means of directly correcting this in realistic boxed wastes due to rapid thermalization of epi-thermal neutron within the matrix itself. Indeed, this realization is one reason why the chamber wall construction can be made more compact and light weight - part of the bulk in chambers from the CTEN stable is needed to 'hold-up' in time the epi-thermal component in an attempt to achieve better penetration and quantification of lumps in certain conditions. However the imaging capability of IBIS can be used to demonstrate whether the signal is confined to a single voxel or not. If the source is clearly not localized but is instead dispersed across several voxels then the normal worst case assumption (i.e. that all the fissile mass is present as a single spherical lump of metal) can be relaxed. This expands the dynamic envelope considerably. IBIS offers the potential of achieving both good matrix and good spatial corrections. In this case the rule of thumb often quoted for $\mathrm{Pu}$ assays that one would expect the presence of lumps to manifest themselves in the observation that the mass obtained from the passive neutron count will exceed that from the passive gamma-ray count which in turn will exceed that from the active interrogation is more likely to hold true. Simple rules such as this can readily be incorporated into an export system tool added on to the back end of the data analysis to assist with data interpretation. 


\begin{tabular}{|l|l|r|}
\hline & Canberra Industries, ACK\# D0043 & 33296 B \\
\hline Created: 29-September-2000 & IBIS Project & Revision B \\
\hline Revised: 13-January-2003 & Preliminary Design Study & Page 7 of 156 \\
\hline
\end{tabular}

- In isolation the active system does not, except under special circumstance, allow $U$ and $\mathrm{Pu}$ to be co-assayed dispite having what appears to be sufficient measured parameters availabie (prompt Total; prompt coincidences; delayed neutrons). In mixed $\mathrm{U}, \mathrm{Pu}$ streams the principle method for assaying $\mathrm{Pu}$ is by the PNCC. $\mathrm{U}$ is inferred by a difference calculation. Unless assumptions to do with co-location (eg if the $\mathrm{Pu}$ is known to be within spent $\mathrm{U}$ fuel or chemically mixed such as the case with MOX), the absence of lumps, knowledge of isotopics, etc., are valid, this will result in large uncertainties in the mass of $U$. However, the gamma-ray system can provide an indication of when the two elements are present in abundance and the passive neutron capability of the BSNS is sufficient to provide an independent measurement of plutonium above a few $10^{\prime} \mathrm{s} \mathrm{mg} \mathrm{level} \mathrm{of}{ }^{240} \mathrm{Pu}$, This is the result of the passive detection efficiency being close to $20 \%$ and the coincidence gate utilization factor being substantially greater than 0.5 due to the fact that all of the efficiency is achieved using $\mathrm{Cd}$ covered fast neutron detector packages which are insensitive to long lived thermal neutrons. In general the sensibility must be commensurate with the practical needs of the assay but in addition to this assay accuracy must not be overly compromised by the desire to achieve high sensitivity.

- Generic methods for gross matrix corrections have been established by groups at LANL and Harwell. These are expected to be directly transferable to box counters. The effort is not so much in the development of the formalism as in the determination of the coefficients to be used in that formalism and the subsequent empirical demonstration of performance. The effort needed to do this is substantial. If a production version of the equipment were to differ from the prototype this activity would have to be repeated. It is therefore important for the design to match the need from the on-set.

- IBIS offers novelty in the way matrix information can be derived for the correction of the passive and active neutron assays. In addition to an array of flux monitors it is proposed to incorporate a Cf add-a-source system. The two approaches are complementary and both offer scope for extracting coarse spatial information. Furthermore the neutronic properties map can be modulated by the gamma-ray transmission map in an effort to refine the coefficients in the response matrix. Important design criteria are the provision of multiple views of the matrix in order to permit data driven (as opposed to algorithmic driven) analysis options which encapsulate spatial information.

- IBIS is a suite of techniques each designed to permit flexible data taking. This opens up new avenues of research, development and demonstration centered around rigorous integration in software of complimentary information.

- Detailed Monte Carlo models of the assay chamber benchmarked to experimental data will allow the simplified calibration and allow the calibration to be extended over a large number of matrix types and source distributions. Many hundreds of simulations have been performed as part of this study and to facilitate this ways of partially automating the process were developed. This can be developed further. 


\begin{tabular}{|l|l|r|}
\hline & Canberra Industries, ACK\# D0043 & 33296 B \\
\hline Created: 29-September-2000 & IBIS Project & Revision B \\
\hline Revised: 13-January-2003 & Preliminary Design Study & Page 8 of 156 \\
\hline
\end{tabular}

\section{Commercialization Path}

- The route to commercialization of an integrated box interrogation system is not well defined. The DOE has not formulated a clear demand profile. This uncertainty makes it difficult for a commercial organization to invest with the confidence and prudence its stakeholders' demand. By delaying commitment of Engineering Design resources several benefits accrue. First it allows the NDA waste assay community to consider its position through the Interface Working Group and other foram. The final IBIS design can then be sure of addressing the appropriate needs and having a receptive market place. Second it reduces some of the technical risk associated with the program by, for example, allowing the development work associated with the PATRM/PCI module to be completed prior to being required. As of January 2003 (some 18 months later than anticipated) the development of the PATRM/PCI had not progressed beyond the design phase and reprioritization of resources within LANL make it unlikely that a prototype unit could be readied in less than 6 months even given the commitment to proceed. In the mean time other commercial suppliers have entered the field and it may be worthwhile to review what is available from the before proceeding. Third, greater resource flattening across Canberra Industries and LANL can be achieved by serializing activities which otherwise would have had to have been performed in parallel in order to meet the original time scale. An example of this is the generation and testing of software and analysis routines which can be developed before-hand on an existing Passive Active Neutron system at LANL. This improves consistency, simplifies communication and minimizes duplication within the project. Forth, it allows for experience gained by Canberra Industries in operating the CWAM system at Oak Ridge and in designing the combined DDA/PNCC/HRGS system for the AMWTP at INEEL to be factored into the final design. The operational requirement for the AMWTP means that the delivery date on the equipment is only of the order of 14 months away. If the build and calibration phases of IBIS were to take place after those of the AMWTP it is anticipated that the IBIS design would benefit from any lessons learned. Examples of where the benefits may be greatest include operational experience and interfacing of the neutron generator; control, data acquisition and user interface software; data integration analysis.

- To provide on going system development and support for a product in the field a commercial vendor requires full access to the software development tools and source code. Only in this way can the product life-cycle be effectively managed in a way that does not rely solely on one individual. Under the proposed division of work between Canberra and LANL several issues of ownership and use remain to be settled. 


\begin{tabular}{|l|l|r|}
\hline & Canberra Industries, ACK\# D0043 & 33296 B \\
\hline Created: 29-September-2000 & IBIS Project & Revision B \\
\hline Revised: 13-January-2003 & Preliminary Design Study & Page 9 of 156 \\
\hline
\end{tabular}

\section{Abstract}

Canberra Industries has won the tendered solicitation, INEEL/EXT-99-00121 for boxed waste Nondestructive Assay Development and Demonstration. Canberra will provide the Integrated Box Interrogation System (IBIS) which is a suite of assay instrumentation and a data reduction system that addresses the measurement needs for Boxed Wastes identified in the solicitation and facilitates the associated experimental program and demonstration of system capability.

The IBIS system will consist of the next generation CWAM system, i.e. CWAM II, which is a Scanning Passive/Active Neutron interrogation system which we will call a Box Segmented Neutron Scanner (BSNS), combined with a physically separate Box Segmented Gamma-ray Scanning (BSGS) system. These systems are based on existing hardware designs but will be tailored to the large sample size and enhanced to allow the program to evaluate the following measurement criteria:

- Characterization and correction for matrix heterogeneity

- Characterization of non-uniform radio-nuclide and isotopic compositions

- Assay of high density matrices (both high-Z and high moderator contents)

- Correction for radioactive material physical form - such as self shielding or multiplication effects due to large accumulations of radioactive materials.

- Calibration with a minimal set of reference standards and representative matrices.

This document summarizes the conceptual design parameters of the IBIS and indicates areas key to the success of the project where development is to be centered. The work presented here is a collaborative effort between scientific staff within Canberra and within the NIS- 6 group at LANL. 


\begin{tabular}{|l|l|r|}
\hline & Canberra Industries, ACK\# D0043 & 33296 B \\
\hline Created: 29-September-2000 & IBIS Project & Revision B \\
\hline Revised: 13-January-2003 & Preliminary Design Study & Page 10 of 156 \\
\hline
\end{tabular}

\section{Introduction}

The DOE has awarded Canberra Industries a "Co-operative Agreement" under 10 CFR 600 Financial Assistance Rules for the development of the Integrated Box Interrogation System, IBIS. IBIS will provide a suite of assay instrumentation and a data reduction system that addresses the needs identified in INEEL/EXT-99-00121 [Becker, Mcllwain, and Connolly, 1999] for the measurement of $\mathrm{CH}$-TRU. The system offers numerous advantages over previously designed crate counting systems, and will undoubtedly stretch the current state-of-the-art. The award was made following the tendered solicitation DE-PS07-991D13812 [Solicitation DE-PS07991D12812].

The IBIS concept centers around the acquisition and analysis of position sensitive data to provide significant reduction in the error contribution from heterogeneous matrix and source distributions. The IBIS extends technologies developed for other applications and addresses interference effects of large crates.

The overriding goal of the project is to design, fabricate and demonstrate a nondestructive assay system for large crate sized samples offering enhancements over existing technologies. The IBIS system will consist of the next generation Crated Waste Assay Monitor (CWAM) system [Becker, Mcllwain, and Connolly, 1999; Melton, Estep, and Perterson, 1999] CWAM II, which is a Scanning Passive/Active Neutron interrogation system which we will call a Box Segmented Neutron Scanner (BSNS), combined with a physically separate Box Segmented Gamma-ray Scanning (BSGS) system. These systems are based on existing hardware designs but will be tailored to the large sample size and enhanced to evaluate the given measurement criteria. Most attempts to tackle these aspects to date have been applied to drum sized or smaller containers. Their usefulness for analysis of boxed waste has not yet been validated.

It is expected that the economic, environmental and operational benefits for the overall US domestic industry as a result of this effort will include:

1. A significant increase in the fraction of boxed TRU waste amenable to direct assay with acceptable accuracy. This will result in less provision for monitoring and interim storage pending ultimate disposal at the Waste Isolation Pilot Plant (WIPP).

2. Ability to assay both uranium and plutonium in a range of containers to meet data quality objectives for waste disposal at DOE low-level waste disposal sites.

3. Ability to screen a large fraction of the boxed waste at the local and/or state set sanitary/LLW fiducial for disposal in sanitary landfills. 


\begin{tabular}{|l|l|r|}
\hline & Canberra Industries, ACK\# D0043 & 33296 B \\
\hline Created: 29-September-2000 & IBIS Project & Revision B \\
\hline Revised: 13-January-2003 & Preliminary Design Study & Page 11 of 156 \\
\hline
\end{tabular}

4. A combined instrument that offers both high sensitivity and accuracy. Improved accuracy and sensitivity result in a reduced probability of sending either LLW to WIPP or LLW to a sanitary landfill. Reducing measurement error bars also has the added benefit of being able to combine more containers per TRU-PACT in one shipment thus reducing shipping cost.

5. Significantly reducing manual effort in expert data review through automation of quality assurance task by databasing data files, methods, and results of every analysis.

6. Faster clean-up of temporary storage areas that now hold boxed waste generated from US weapons programs because fewer boxes will need to be re-packed into drum sized containers in order to meet assay data quality objectives.

7. A mobile instrument that can service the needs of sites and facilities that generate only relatively small volumes of boxed waste. This is cost effective because dedicated expensive capital equipment and ancillary buildings are not required. Furthermore, the same rigorous assay and quality assurance can be applied across sites and can be applied to large volume decommissioning debris. A mobile instrument would reduce the need for bespoke or ad hoc expert review and would reduce the need for intrusive sampling with its associated need for special equipment and the inevitable radiological risks that result. 


\begin{tabular}{|l|l|r|}
\hline & Canberra Industries, ACK\# D0043 & 33296 B \\
\hline Created: 29-September-2000 & IBIS Project & Revision B \\
\hline Revised: 13-January-2003 & Preliminary Design Study & Page 12 of 156 \\
\hline
\end{tabular}

\section{Program Structure}

The contract is essentially a "best effort" contract where funding continues on a yearly and milestone basis as long as substantial progress is realized. The agreement calls for cost sharing with the Government's contribution capped at $\$ 2 \mathrm{M}$.

The estimated duration of the program at the time of inception was 30 months. For various reasons, to be discussed later, the schedule may be extended. Overall it is considered that this will strengthen the project and reduce risks.

The project is organized into three interlinked phases.

The program of work will accommodate the design, build, test and supply of the gamma and neutron box counters with an integrated system of data accumulation and analysis for position dependent data. The design phase (Phase 1) is reported here. In brief we have carried out a series of numerical simulations to determine the optimum sample voxel size practical and useful for data accumulation and later combined gamma/neutron analysis. This has defined the hardware needs and data integration techniques. Through this work, the choice of detector layouts and crate scanning options in both the neutron and gamma counters has been scoped - although this requires some further detailed transport code simulations to finalize the selection. To cope with the increased quantity and types of data, revised data acquisition, storage protocols and software needs are required. The options under consideration for the system architecture are reviewed. A key driver is the objective of mounting the BSNS on a trailer so that it can be readily moved and re-deployed.

The build phase (Phase 2) activities will include preparation of fabrication drawings, and fabrication of the systems. The systems data acquisition and integration software design will begin. Preliminary sub-system characterization will be performed at Canberra facilities in Meriden, CT. More extensive system measurements will be carried out at LANL, NM. Preparatory work on data acquisition and data analysis engines will take place throughout Phase 2.

The test phase (Phase 3) will use source placement studies in appropriate matrices to benchmark the extensive set of Monte Carlo studies carried over from the design phase, which will form the main calibration database for the IBIS system. For the BSNS, the utility of the various flux and cavity monitors and related correction techniques will be assessed and improved by the data, the calculations, and, by the optimization methods used in the CTEN and CWAM developments. The analysis techniques will also be developed further. The objective is to identify efficient algorithms for the neutron and gamma systems to work together in an optimized position-mapped analysis. The measurements and in large the part analysis techniques development described above during Phase 3 for the neutron portion of the IBIS will be performed at LANL. Finally a demonstration of the system capability will be made using waste form surrogates characteristic of 4 matrix types and 4 crate types, and reports will be delivered as required, recording the project 


\begin{tabular}{|l|l|r|}
\hline & Canberra Industries, ACK\# D0043 & 33296 B \\
\hline Created: 29-September-2000 & IBIS Project & Revision B \\
\hline Revised: 13-January-2003 & Preliminary Design Study & Page 13 of 156 \\
\hline
\end{tabular}

information and the proven IBIS performance. It is expected that all of the algorithmic developments may not be fully integrated into the analysis software at the completion of Phase 3 . Rather, these will probably be applied in a post-processing step utilizing programmed spreadsheets.

The IBIS will be transported to a DOE facility selected by the MWFA for final demonstration of the system performance. It is anticipated that the system will then be ready to measure containers destined for WIPP, and may be useful for the Performance Demonstration Program (PDP) tests [DOE/WIPP-069; Section 9 Revision Interim Change, 1996]. At this time a short duration demonstration phase is anticipated. This will aim to show under what conditions the following measurement criteria can be obtained:

- Detection Level for Uranium $<35 \mathrm{pCi} / \mathrm{g}$ - a value representative of land fill disposal criteria in $\mathrm{NM},(250 \mathrm{pCi} / \mathrm{g}-\mathrm{CO})$

- Detection Level for Plutonium $<10 \mathrm{nCi} / \mathrm{g}$ - a value representative of $\mathrm{LLW}$ disposal criteria

- Detection Level for Plutonium $<60 \mathrm{nCi} / \mathrm{g}$ - a value which would give confidence that the transuranic waste fiducial of $100 \mathrm{nCi} / \mathrm{g}$ could be policed with confidence

- Assay precision and bias meet requirements for WIPP acceptance

- Assay identifies and corrects errors due to matrix heterogeneity

- Assay identifies and corrects errors due to source heterogeneity

- Assay identifies and corrects errors due to high density matrices

It is a further goal to demonstrate that these performance objectives can be met with a limited set of calibration standards and matrices of different kinds.

The core project team is a collaboration between Canberra Industries personnel (including Harwell Instruments Ltd, a wholly owned Canberra subsidiary based in the United Kingdom) and LANL staff from the Advanced Nuclear Technology Group NIS-6. This combination is a very powerful one in that it combines the in-depth research strengths of the National Laboratory with the commercial drive of the private sector.

The participation of the Los Alamos National Laboratory's NIS-6 group is essential to the successful completion of this project. The NIS-6 development of the CTEN and CWAM technologies provides the basis for the treatment of the sample heterogeneity in the BSNS analysis. The proposed BSNS system provides the following features not currently available for crate or box assay in the private sector:

- List Mode Acquisition and Analysis 


\begin{tabular}{|l|l|r|}
\hline & Canberra Industries, ACK\# D0043 & 33296 B \\
\hline Created: 29-September-2000 & IBIS Project & Revision B \\
\hline Revised: 13-January-2003 & Preliminary Design Study & Page 14 of 156 \\
\hline
\end{tabular}

- A high sensitivity yet accurate active neutron measurement for the measurement of uranium and plutonium at low levels; a high accuracy passive neutron measurement for plutonium TRU waste; a scanning passive high resolution gamma-ray spectrometry station for the determination of isotopic information

- Trailer mounted configuration

- Scanning Neutron Generator

- Matrix heterogeneity corrections

LANL NIS-6 will participate under a Work for Others agreement to provide physics support and design input for the fabrication of the BSNS system. The mechanical engineering and drafting work will be completed by Canberra engineers. Many of the analysis algorithms for the active neutron measurements, source heterogeneity correction, and matrix correction are to be developed by LANL personnel. Engineering functions will be under the direction of, and be performed by, Canberra personnel with input from LANL scientists. Software design and compilation, including computer communications with hardware, Programmable Logic Controllers (PLCs), and other electronic media, will be largely developed by LANL personnel with support from Canberra Instruments. System calibration and tests will be performed by LANL personnel with extensive input from Canberra Instruments Project organization and management will be under the control of Canberra.

The BSGS will be designed and developed primarily by Canberra but with LANL NIS-6 providing detailed software and calibration support. 


\begin{tabular}{|l|l|r|}
\hline & Canberra Industries, ACK\# D0043 & 33296 B \\
\hline Created: 29-September-2000 & IBIS Project & Revision B \\
\hline Revised: 13-January-2003 & Preliminary Design Study & Page 15 of 156 \\
\hline
\end{tabular}

\section{The System}

The IBIS consists of two sub-systems whose data will be combined to derive the final results. The first sub-system is a large gamma counter capable of assaying large crates of radioactive waste. It will determine isotopic and tomographic information. Non homogeneity will be identified from segment count-rate data in two orientations each of two planes. The second subsystem will be a large neutron counter which will assay the crates in both active and passive mode. Assay and imaging results from both sub-systems will be combined to provide an improved result.

The four main functions of the BSGS are:

- Gamma assay of the crate to detect and quantify gamma emitting nuclides.

- Plutonium and uranium isotopics measurements, both localized and sample averaged.

- Matrix heterogeneity measurement.

- Source non uniformity measurement.

To address the MWFA needs the BSGS includes the following innovations in crate counting:

- Matrix heterogeneity detection and correction using active (transmission source) and passive gamma-ray measurements.

- Plutonium isotopic and uranium enrichment measurements for localized and diffuse source distributions using the MGA [Gunink, et al., 1994] and MGAU [Gunink 1990; Gunink and Ruhter, 1990] codes.

- Quantitative assay of many radionuclides that are not detectable by neutron interrogation. Results of the analysis are reported in units of plutonium mass, activity of individual isotopes, total alpha activity, total activity, fissile gram equivalent, and power; i.e. the criterion of interest for disposal of TRU waste at the WIPP facility.

- Identification and reporting of heterogeneous source distributions.

The BSGS solution is strongly based on the successful Canberra Box Counters already deployed in a mobile format for DOE complex assignments. The extensions here arise in additional detectors, their mechanical deployments and their control. The transmission corrections are well founded in the Canberra SGS family, the workhorse of gamma assay in the industry.

The BSNS (Box Segmented Neutron Scanner) solution and its corrections used here are based on Los Alamos National Laboratory's (LANL's) successful Combined Thermal Epithermal Neutron [Melton, Estep, and Hollas, 1999] (CTEN) and CWAM developments. In this case, the 


\begin{tabular}{|l|l|r|}
\hline & Canberra Industries, ACK\# D0043 & 33296 B \\
\hline Created: 29-September-2000 & IBIS Project & Revision B \\
\hline Revised: 13-January-2003 & Preliminary Design Study & Page 16 of 156 \\
\hline
\end{tabular}

measurement chamber is optimized to provide good position dependent information, and to have a sufficient efficiency for passive neutron detection as well as a good active neutron response. Also, initial evaluation and tests have been performed for the advanced algorithms that assess matrix effects, source heterogeneity, self-shielding, and simplified calibration. The neutron system will be designed and fabricated by Canberra with guidance from the LANL NIS-6 group and will integrate the tomographic techniques developed for the CTEN system into the BSNS (Box Segmented Neutron Scanner). The BSNS offers enhanced performance relative to traditional Passive/Active Neutron (PAN) crate counters by providing:

- Detection and mapping of the relevant heterogeneous matrix characteristics, and correction for their effects on the passive and active neutron assay results.

- Correcting both passive and active neutron assay results for non-uniform source distributions

- Integration of the position dependent isotopic information from the BSGS system with the neutron measurements.

- An investigation of where matrix corrections break down due to, for example, shielding effects in dense matrix materials will be performed. The investigation will study where gamma-ray and neutron matrix correction factors can supplement one another and the limitations on the use of correction factors for boxed waste analysis.

- Simplified calibration routines will be examined in the gamma-ray analysis by applying an efficiency calibration over broad energy and matrix density ranges. Neutron calibration will be simplified by a thorough test and characterization program of the neutron system response over a large number of matrix types and source distributions. Numerical modeling will be used to supplement experimental measurements.

In the BSNS multi-position neutron data is accumulated and its analysis is performed in Totals, Reals (coincidence) and multiplicity modes using a list mode analyzer. These advanced technical and analytical features are used to correct for matrix effects, source non-uniformity and selfshielding where possible, and to provide simplified calibration procedures. These effects have previously been identified as deficiencies in existing box NonDestructive Assay (NDA) systems and are also identified as requirements to be addressed in the current solicitation [Becker, Mcllwain, and Connolly, 1999; Solicitation DE-PS07-991D12812].

For the BSNS and BSGS systems, imaging/position correction techniques will be developed to provide information on the makeup and distribution of the matrix and radioactive materials within the sample and amelioration for their effects on the assay result.

A coherent data analysis and integration scheme will be developed that combines the positional and activity measurements from the two systems to provide a single defensible assay result. This combined analysis will provide reduced errors in the reported radionuclide inventory for the sample. 


\begin{tabular}{|l|l|r|}
\hline & Canberra Industries, ACK\# D0043 & 33296 B \\
\hline Created: 29-September-2000 & IBIS Project & Revision B \\
\hline Revised: 13-January-2003 & Preliminary Design Study & Page 17 of 156 \\
\hline
\end{tabular}

\subsection{Characterization and Correction for Matrix Heterogeneity}

Matrix heterogeneity generally impacts gamma-ray systems far more than neutron systems but in each case the matrix heterogeneity must be accounted for in order to provide an accurate assay result.

For gamma-ray assay, relatively small concentrations of high- $Z$ materials can significantly attenuate the gamma-ray signal from a compact source. In addition to correcting for the average matrix effects, gamma-ray systems must also identify these high- $Z$ concentrations as a potential source of error and correct for them. The BSGS will provide data on matrix composition as a function of position by use of a scanning transmission source. The sample's gamma-ray assay data is also acquired using a scanning system so that the areas of potential concern can be analyzed in more detail. While a complete tomographic analysis using the gamma-ray system is not envisioned at this time, the assay data set mapped over the crate can be used to significantly reduce the assay errors.

The effect of matrix heterogeneity on neutron assay is more complicated but the "significant volume" is much larger. For example, the gamma-ray signal can be reduced by an order of magnitude by only a few mm of lead, while in neutron counting the non-uniformity must generally be 10's of centimeters in extent in order to have significant impact. However, there are some combinations of moderator, reflector and absorber than can introduce significant localized reduction in response. These must be identified and accounted for in the analysis. The BSNS technology will use the tomographic techniques developed for the CTEN and CWAM systems, providing a map of neutron response of the sample that can be used to improve accuracy through the provision of position-dependent corrections. Based on physical / physics arguments a target volume element (voxel) size of approximately one foot is being targeted.

\subsection{Characterization of Non-Uniform Radio-Nuclide and Isotopic Compositions}

The BSGS will perform high resolution spectroscopy assay at regular intervals across the surface of the crate. At each location the spectra will be analyzed using MGA and MGAU for plutonium and uranium isotopics respectively. In addition the data will be analyzed in conjunction with the transmission data to provide a position dependent quantitative assay of the crate similar to that obtained in a standard SGS system. Even without tomographic image reconstruction techniques the basic benefits of bilateral counting in order to improve geometrical averaging is well established. The BSGS allows this to be applied either column by column or segment by segment.

The BSNS can, using developments of the CTEN and CWAM tomographic analysis, provide information on the locations of concentrations of fissile and spontaneously fissionable materials. The neutron response at each location is corrected for position effects caused by changes in moderation/absorption as a function of depth into the sample. The isotopics data obtained from 


\begin{tabular}{|l|l|r|}
\hline & Canberra Industries, ACK\# D0043 & 33296 B \\
\hline Created: 29-September-2000 & IBIS Project & Revision B \\
\hline Revised: 13-January-2003 & Preliminary Design Study & Page 18 of 156 \\
\hline
\end{tabular}

the BSGS can then be integrated with the BSNS results to account for variations in isotopics as a function of position.

\subsection{Assay of Difficult Matrices}

The neutron and gamma assays complement each other in that the matrix materials that create the most assay difficulties in the one system often pose less problem for the other. While there will inevitably be samples that can not be assayed due to matrix shielding issues, the combination of these technologies will reduce that occurrence. For instance, while a gamma-ray system cannot accurately quantify the plutonium content of a densely packed steel container, this matrix introduces little error into the assay by passive coincidence counting. Conversely, light heterogeneous matrices containing neutron absorbers which are problematic for neutron counters, pose little problems for gamma systems.

In similar vein active and passive assays often display contrary systematic biases as a function of moderator density. By forming appropriately weighted averages assays which are more accurate. can be obtained.

The positioning information obtained from the neutron and gamma-ray measurements will allow position dependent correction factors to be used when there is a statistically significant source signal to which they can be applied. This will minimize instances where the shielding effects effectively mask the signal (i.e. the result will be lower detection limits than would be achieved with a single system).

The synergy between the passive gamma, passive neutron and active neutron capabilities of IBIS will be explored in later sections of this report. 


\begin{tabular}{|l|l|r|}
\hline & Canberra Industries, ACK\# D0043 & 33296 B \\
\hline Created: 29-September-2000 & IBIS Project & Revision B \\
\hline Revised: 13-January-2003 & Preliminary Design Study & Page 19 of 156 \\
\hline
\end{tabular}

\section{Box Segmented Gamma-Ray Scanner}

The BSGS solution is strongly based on the successful Canberra Box Counters already deployed in several assignments, such as Envirocare in Utah and at the Vandellos I site in eastern Spain. The extensions here arise principally from additional detectors, their mechanical deployments and control, and from the addition of transmission sources for density profiling. The system is designed to accommodate a vast range of crate sizes, including, in particular:

- The Standard Waste Box (approximate nominal OD: 37 inches height $x 54$ inches width $x 71$ inches length)

- B-25 Box (approximate nominal OD: 52 inches height $x 47$ inches width $x 73$ inches length)

- Plywood Box [SAND Type-1] (approximate nominal OD: 48 inches height $\mathrm{x} 48$ inches width $\mathrm{x} 84$ inches length)

- Corrugated Metal Box (approximate nominal OD: 54 inches height x 54 inches width $\times 88$ inches length)

The system will also accommodate, as an extreme, containers having 58 inches outside height and width the length is a simple scan so that a wide range of boxes can be accomodated. The system will assay the sample box by counting at many detector positions (i.e. segments) along the four long sides of the box. At each segment, transmission data will be collected, thereby providing a density profile of the sample matrix, and emission (i.e. passive) data will be collected, providing a profile of the activity distribution inside the container.

The nominal box dimensions of 58" $\times 58$ " $\times 92$ " used here for design purposes is not a limiting case. Boxes $60 "$ x $60 "$ x 96 " could readily be catered for in the Engineering phase.

The system is designed to allow flexibility in the choice of software for motion control, data acquisition, and data analysis. At this time, two options are being reviewed:

- Control, acquisition, and analysis software developed by Canberra Industries. This software package will be based on Canberra's successful Genie-2000 acquisition and analysis platform. Many of the algorithms needed for the IBIS BSGS do not lend themselves to seamless incorporation into Canberra's industry standard NDA-2000 waste assay system software. However, most of the code and engineering embodied in NDA-2000 will be used in the IBIS NDA-2000 software. This will save development time and increase reliability by utilizing previously proven and tested code.

- Control, acquisition, and analysis software based largely on the TGS codes developed at LANL NIS-6. The design of the system hardware is such that it closely mimics that of the highly regarded NIS-6 TGS system. With minimal modifications, the existing TGS code can be modified to dovetail with the IBIS BSGS hardware design. 


\begin{tabular}{|l|l|r|}
\hline & Canberra Industries, ACK\# D0043 & 33296 B \\
\hline Created: 29-September-2000 & IBIS Project & Revision B \\
\hline Revised: 13-January-2003 & Preliminary Design Study & Page 20 of 156 \\
\hline
\end{tabular}

These issues will be discussed in more detail below.

\subsection{Mechanical Design}

The gross structure of the BSGS consists of five modules - two vertical detector / transmission drive assemblies, one on each side of the box; two horizontal detector / transmission drive assemblies, one each above and below the box; and a conveyor system which moves the box through the counting equipment. Having detectors above and below the crate in addition to those at the sides provides additional spatial information and opens up greater analysis options. It represents an enhancement over existing designs.

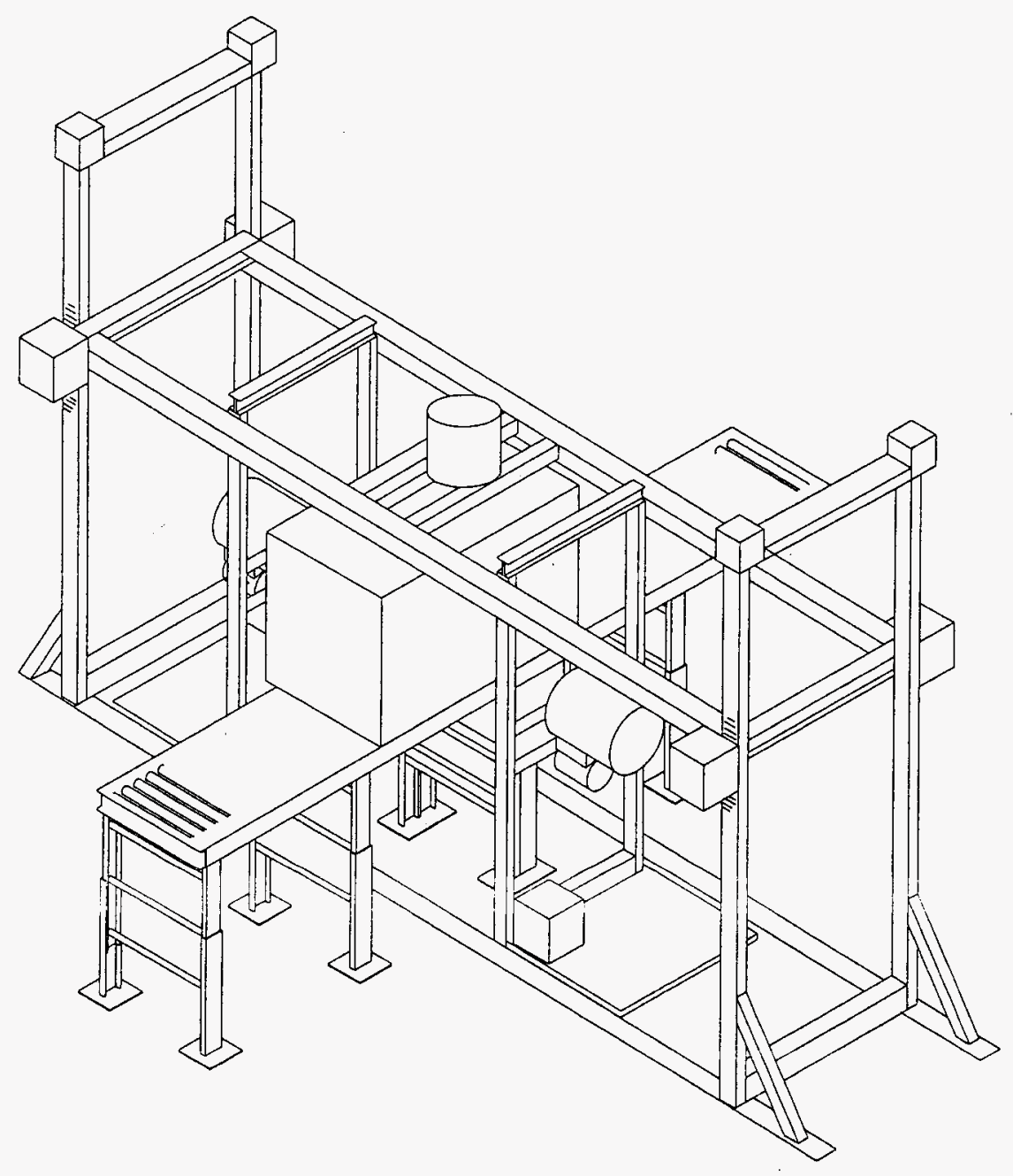

Figure 1. Conceptual drawing of the BSGS. The system employs four detectors (one each on the top, bottom, and sides) and two transmission sources (one on the bottom and one on the side).

The container box moves on a conveyor through the detector mechanism 


\begin{tabular}{|l|l|r|}
\hline & Canberra Industries, ACK\# D0043 & 33296 B \\
\hline Created: 29-September-2000 & IBIS Project & Revision B \\
\hline Revised: 13-January-2003 & Preliminary Design Study & Page 21 of 156 \\
\hline
\end{tabular}

The two vertical detector / transmission drive assemblies are positioned on either side of the box and remain stationary with respect to the ground during assay. Each drive assembly rests on rails which run perpendicular to the motion of the box; this is to allow the two assemblies to be positioned towards/away from the box so as to accommodate different box widths. Each drive assembly is equipped with a lead housing. One housing contains one HPGe detector; the other contains one HPGe detector and one transmission source. The housing serves as a collimator and side shield for the detector and as a collimator and radiation shield for the transmission source. Each housing moves up and down along the length of the vertical drive assembly via an automatically controlled motor and worm drive. When placed in position, the two housings oppose one another across the width of the box. During the transmission pass, the collimated transmission source of one housing shines into the detector on the opposite housing. During the passive emission pass, the two detectors oppose one another. The vertical motion of the housings allows them to scan the height of the box.

The top horizontal detector drive assembly is supported as a gantry perpendicular to and straddling the path of the box's motion. It remains stationary relative to the ground during assay. The vertical position of the gantry is adjustable to accommodate different box heights. The horizontal drive assembly is equipped with a lead housing containing one detector, similar to that used on the vertical assemblies. This housing moves left and right across the width of the horizontal drive via an automatically controlled motor and worm drive, thereby allowing it to scan the width of the box.

The bottom horizontal detector / transmission drive assembly rests on the ground and remains at a fixed height. The box, rolling on the conveyor system, moves over this assembly. This assembly is equipped with a lead housing, containing one detector and one transmission source, and motorized worm drive identical to that in the top assembly. When placed in position, the top and bottom housings oppose one another across the height of the box. The collimated transmission source shines into the top detector during the transmission pass, and the bottom detector opposes the top detector during the passive emission pass. The left/right motion of the housings allows them to scan the width of the box.

During assay, the four detector / transmission housings are automatically driven to scan along the width and height of the box.

The conveyor system consists of a set of driven rollers which propel the box through the rectangular framework of drive assemblies, much like an automobile propelled through an automatic car wash. There is a gap in the rollers to accommodate the line of sight for the bottom detector / transmission housing. The speed and stop/start intervals of the conveyor motion allows the detectors and transmission sources to scan along the length of the box. The conveyor support and drive mechanisms will be fabricated so as to support the heaviest expected box with an appropriate safety margin. 


\begin{tabular}{|l|l|r|}
\hline & Canberra Industries, ACK\# D0043 & 33296 B \\
\hline Created: 29-September-2000 & IBIS Project & Revision B \\
\hline Revised: 13-January-2003 & Preliminary Design Study & Page 22 of 156 \\
\hline
\end{tabular}

At Canberra, trailer-mounted systems are usually installed in typical 'moving van' trailers. These have lighter frame construction than the more common cargo trailers, and are provided with large side doors. The dimensions of these trailers is roughly 10 feet high and 8 feet wide. The system could be fit into such a trailer, with the conveyor (and hence the motion of the box) extending out the side doors, perpendicular to the length of the trailer. The 8-foot width of the trailer does not pose a serious design challenge, as the footprint of the system is expected to be narrow (less than 4 feet) along the long axis of the box. The height of the trailer would accommodate the system if the bottom drive assembly is suspended beneath the floor of the trailer. In this case, however, maintenance and assembly access to the top gantry and bottom drive would be more restricted than if the system were assembled as a stationary, standalone device. Assembled this way, the system would still require a minimal amount of time for partial disassembly and would fit inside a typical shipping vehicle for transportation. At this time, it is intended, for demonstration purposes, to design and build the BSGS as a stationary, standalone system. The possibility of installing the system permanently in a trailer will be evaluated, but will not be implemented at this time in order to stay within spending limits.

\subsection{HPGe Detectors and Collimator Design}

For the BSGS system, the four HPGe detectors will each be a Canberra model BE3825. With a frontal area of $38 \mathrm{~cm}^{2}$ and a thickness of $25 \mathrm{~mm}$ this detector model is one of the largest (i.e. highest efficiency) in Canberra's unique Broad Energy Germanium (BEGe) Detector line [Tench, 1997] . BEGe detectors were selected for this application for several reasons. BEGe's provide excellent resolution over a wide energy range. The BEGe is designed with an electrode structure that enhances low energy resolution, and it is fabricated from select germanium having an impurity profile that improves charge collection (thus resolution and peak shape) at high energies. The resolution at low energies is equivalent to that of our Low Energy Germanium Detector and the resolution at high energy is comparable to that of good quality coaxial detectors. This ensures good resolution and peak shape over the entire mid-range which is particularly important in analysis of the complex spectra from uranium and plutonium. Further, the BEGe has a short, fat shape which is specifically chosen for optimum efficiency below $1 \mathrm{MeV}$ for typical sample geometries. In addition to higher efficiency for typical samples, the BEGe exhibits lower background than typical coaxial detectors because it is more transparent to high energy gamma rays from cosmogenic background and from naturally occurring radioisotopes such as ${ }^{40} \mathrm{~K}$ and ${ }^{208} \mathrm{Tl}$ (Thorium).

To make optimal use of space and to minimize periodic maintenance, each detector will be kept cold via Canberra's Cryoelectric II Electrically Refrigerated Cryostat. The refrigerator units have a small footprint and can be secured near the assay system.

The lead housing serves as a collimator and side shield for the detector, shielding it not only from ambient radiation, but also from the gamma rays emitted from the transmission source which may also be enclosed in the housing. The collimator design is based largely on the standard collimator used in Canberra's Box Counters. The collimator portion of the housing extends along the entire length of the detector, and is 2 inches thick. The front face of the collimator is $5 \mathrm{~cm}$ 


\begin{tabular}{|l|l|r|}
\hline & Canberra Industries, ACK\# D0043 & 33296 B \\
\hline Created: 29-September-2000 & IBIS Project & Revision B \\
\hline Revised: 13-January-2003 & Preliminary Design Study & Page 23 of 156 \\
\hline
\end{tabular}

thick with a 20 degree opening angle (aperture radius at the detector face is $1.8 \mathrm{~cm}$, aperture radius at the collimator face is $3.6 \mathrm{~cm}$ ). This is intended to provide a roughly 1 foot viewing area with appropriate overlap when projected onto the surface of the box from a distance of roughly $40 \mathrm{~cm}$, while still providing illumination to most of the detector crystal volume. In addition, the collimators are provided with cadmium and tin shield plates which can be manually placed over the collimator apertures to reduce count rate from high ${ }^{241} \mathrm{Am}$ concentrations.

We note here for completeness that $\mathrm{NaI}(\mathrm{TI})$ arrays operated in gross or broad ROI counting mode (combined with transmission corrections for matrix compensation) could be used to achieve much higher efficiency ( $100 \mathrm{x}$ say) than the proposed HPGe array at the same cost. This is potentially useful for screening applications - especially when the relative composition of radionuclides is reasonably well known and constant. However, HRGS gives the best specificity for a general purpose unit.

\subsection{Transmission Sources and Shield Design}

The two transmission sources used in the system are mounted in the lead housings. The source shield portion of the housing design is based largely on the existing design for Canberra's Segmented Gamma Scanner source holder. In designing the source and shield combination, several considerations were taken into account. It was desirable to choose the source strength, gamma-ray energy, and gamma-ray intensity so as to provide a high degree of transmission through the containers and the matrix materials inside them. This would provide good counting statistics on the transmission measurements and would ensure that reliable transmission information would be obtainable even for fairly dense matrices. It was necessary, however, to also provide adequate radiation shielding for personnel safety and to minimize the additional background and interference observed in the HPGe detector mounted in the same housing just inches away from the source.

The sources contained in two of the four housing are $10 \mathrm{mCi}$ each of ${ }^{60} \mathrm{Co}$. This nuclide was selected because it emits two strong high-energy lines $(1173 \mathrm{keV}$ and $1333 \mathrm{keV}$, both at roughly $100 \%$ intensity). It would be desirable to utilize lower energy lines, such as the $122 \mathrm{keV}$ line from ${ }^{57} \mathrm{Co}$, or the $122 \mathrm{keV}$ and $344 \mathrm{keV}$ lines from ${ }^{152} \mathrm{Eu}$, which are closer in energy to the typical lines of interest for uranium and plutonium assay. It was determined, however, that such low energy transmission lines would have a poor observablity through more dense matrices. $10 \mathrm{mCi}$ provides sufficient source strength to be seen through the majority of container sizes and material types/densities with a transmission acquisition time of 5 minutes per segment. The expected count rate was estimated for the system, taking into account the efficiency of a BE3825 and the attenuation in the matrix material (all of these quantities are averaged over the expected range of matrix material types and over the two ${ }^{60} \mathrm{Co}$ gamma-ray energies). The resulting data are presented in the plot shown in Figure 2. Two specific examples can be cited for the estimated single-line transmission peak area in a 5-minute single-segment acquisition:

48 inch box width, density of $0.5 \mathrm{~g} / \mathrm{cm}^{3}: 51000$ counts 


\begin{tabular}{|l|l|r|}
\hline & Canberra Industries, ACK\# D0043 & 33296 B \\
\hline Created: 29-September-2000 & IBIS Project & Revision B \\
\hline Revised: 13-January-2003 & Preliminary Design Study & Page 24 of 156 \\
\hline
\end{tabular}

54 inch box width, density of $1.2 \mathrm{~g} / \mathrm{cm}^{3}: 140$ counts

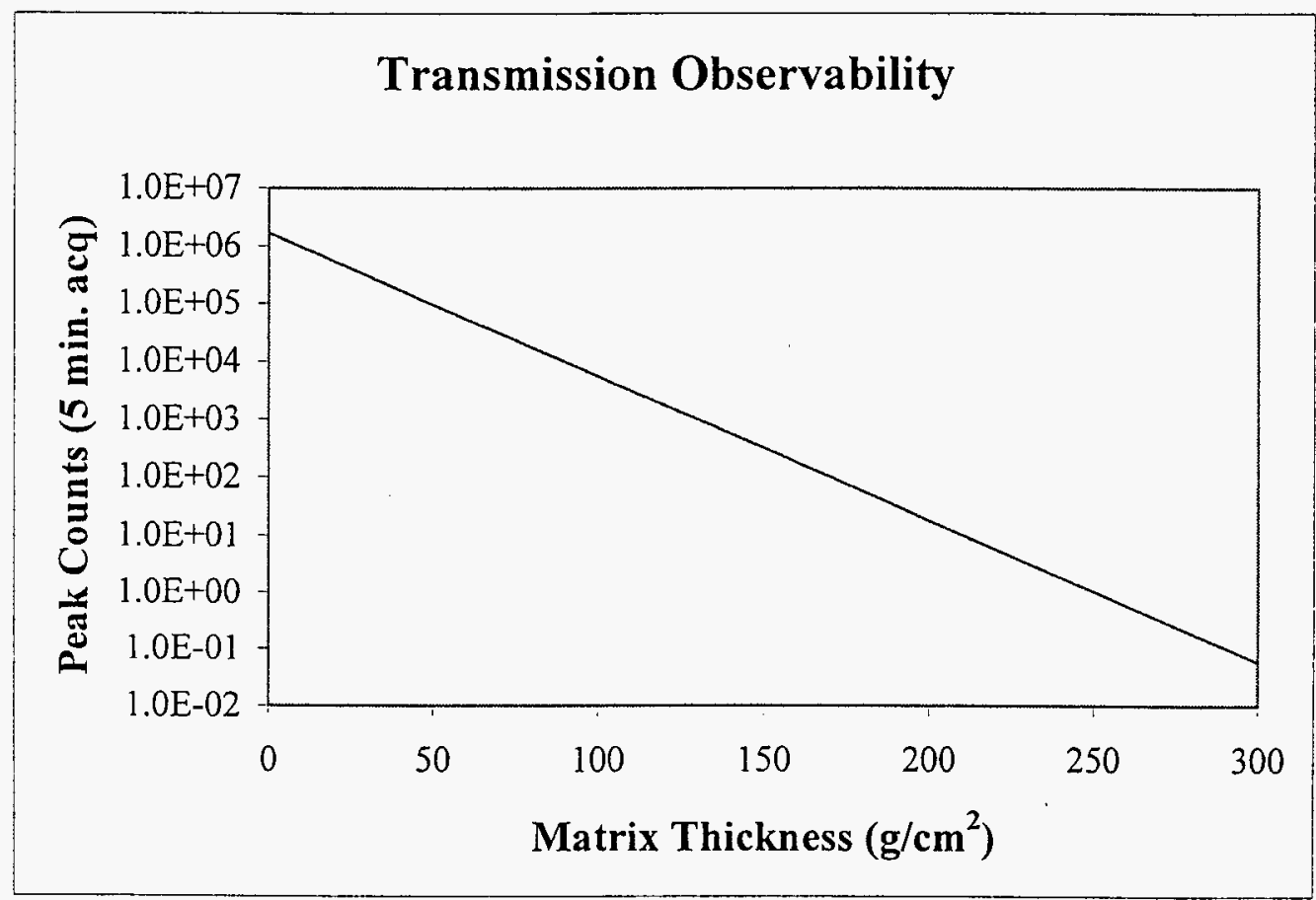

Figure 2. Transmission Peak Area as a Function of Sample Thickness. The peak area shown is for a 5 minute acquisition, and represents a single-peak average of the two ${ }^{60} \mathrm{Co}$ lines. The matrix was taken as an average of the expected common material types (e.g. soil, debris).

The source shield is a cylindrical lead container, 10 inches in diameter and 10 inches long. A 1/4 inch diameter hole is bored in one circular face to a 5 inch depth. The source is mounted at that depth in the hole, which thus serves as the source collimator. Mounted on the front of the shield is a 3 inch thick Tungsten shutter, which is under automatic control to shine or occlude the source gamma rays through the sample container. This design provides adequate radiation shielding for personnel safety. It is estimated that the exposure rate at any surface of the shield is less than $1 \mathrm{mR} / \mathrm{hr}$. On the two housings that have both a detector and a source, the two units (the cylindrical detector collimator and the cylindrical source shield) are mounted side-by-side. It is also necessary, however, to augment the shielding to minimize the additional background and interference observed in the HPGe detector from the source. Therefore, an additional lead block is provided between the shield and collimator. This increases the total path length to 12 inches of lead between the source and the surface of the detector. Thus configured, it is estimated the count rate observed in the neighboring HPGe detector is less than 0.0001 counts/second for either of the two ${ }^{60} \mathrm{Co}$ lines. In a half-hour single-segment count, this would not be observed in the emission spectrum.

Of interest in the engineering design effort is the weight of the collimators, shields, etc. Rough estimates can be obtained from the gross dimensions of the items already discussed. The detector 


\begin{tabular}{|l|l|r|}
\hline & Canberra Industries, ACK\# D0043 & 33296 B \\
\hline Created: 29-September-2000 & IBIS Project & Revision B \\
\hline Revised: 13-January-2003 & Preliminary Design Study & Page 25 of 156 \\
\hline
\end{tabular}

collimator, of which there are four, is basically a cylindrical tube approximately 14 inches long, with 6 inch inside and 10 inch outside diameters. One such collimator weighs approximately 290 pounds $(130 \mathrm{~kg})$. The transmission source shield, of which there are two, is essentially a 10 inch x 10 inch cylinder, one of which weighs approximately 320 pounds ( $146 \mathrm{~kg}$ ). The two additional lead shield blocks (each placed between the transmission shield and detector collimator) are cubes, 5 inches on a side, which each weigh approximately 50 pounds $(23 \mathrm{~kg}$ ). Combined, the total weight of the shielding and collimators is, then, just under one ton. Existing designs for Canberra's systems, from which the BSGS design draws heavily, routinely handle lifting/moving lead components of these sizes. The elaborate shielding and collimation requirements will not present a difficulty in the construction of the system.

\subsection{Motion Control and Scanning Protocol}

A driving objective behind the mechanical design of the BSGS was flexibility. The left and right side vertical drive assemblies, and the top horizontal drive assembly can be positioned to accommodate a vast range of box sizes. The top and bottom detectors and transmission source can scan left/right over the width of the box. The left and right detectors and transmission source can scan up/down over the height of the box. The conveyor system allows all four detectors and both transmission sources to scan over the length of the box. The intent here is for the assay motion to take place in discrete steps, but it is entirely possible for the system hardware to run a continuous scan instead. The motors which drive the detectors, transmission sources, and conveyor are all intended to be controlled by GE-Fanuc programmable logic controllers (PLCs). Nearly all of Canberra's automated systems use this class of PLCs. In addition to Canberra's lengthy history and success with these PLC units, the GE-Fanuc communication interface is well known and easily programmable. The same is true of the acquisition driver interface. This allows a great deal of software flexibility - the mechanical and acquisition hardware systems are not irrevocably wedded to Canberra software. The use of Canberra software would bring to bear a large ensemble of existing control, acquisition, and analysis algorithms as well as allowing Canberra's team of scientists to develop new algorithms to utilize the full range of detailed data available from the BSGS system. Alternatively, the LANL NIS-6 TGS software could be easily modified to control the motion of the system as well as the acquisition and analysis of the measurement spectra and data. The team of scientists and engineers from Canberra and LANL are currently evaluating the benefits of using Canberra software, LANL TGS software, or a hybrid of both.

\subsection{Scanning Protocols}

The system is designed to scan four sides of the box - top, bottom, left, and right - assumed to be the four "long" sides of the box. The detector collimators are intended to provide coverage of the box faces with the system scanning in discrete steps of one foot in each dimension. Thus, for a B25 box, for example, which has rough dimensions of 4 feet $x 4$ feet $x 6$ feet, each detector will make 24 measurements along it's face of the box. For instance, the top detector will scan in four positions left/right across the top face of the box while the conveyor system will move the box 


\begin{tabular}{|l|l|r|}
\hline & Canberra Industries, ACK\# D0043 & 33296 B \\
\hline Created: 29-September-2000 & IBIS Project & Revision B \\
\hline Revised: 13-January-2003 & Preliminary Design Study & Page 26 of 156 \\
\hline
\end{tabular}

past the detector's line of motion in 6 positions. Thus, a full scan will entail 24 acquisition periods, and will generate 96 spectra.

The system is designed to work in a two-pass mode. One pass will consist of the transmission measurements. The source-bearing housings will be positioned so as to shine the collimated sources into the detectors on the opposite housings. It is expected that 3-5 minutes acquisition per segment will be sufficient to do the transmission measurement. The other pass will consist of the passive emission measurements. In this pass, the source-bearing housings will be positioned so as to place the pairs of detectors (top-bottom, left-right) directly opposite one another. It is expected that the single-segment acquisition time will be between 10 and 30 minutes, depending on the activity and density of the box. Thus a typical full assay will take roughly 8 hours, not including crate handling. If throughput so demanded, additional detectors and/or assay stations can be considered.

It is useful here to describe a simple physical model to illustrate the potential measurement schemes the BSGS system can employ for typical boxes. The scanning geometry is illustrated in Figure 3 . The box is shown with its bottom face aligned with and resting on the positive $x y$ plane. The box is incrementally scanned past the four detectors, $D_{1}-D_{4}$, which define a field of view, or vertical segment, through the box in the yz plane. For mathematical expediency at this stage in setting up a simple interpretational physical model, we imagine the detectors to be distant from the box and their geometrical field of view to be well defined by collimators. Thus in the expressions that follow, we can invoke the far field approximation for attenuation suffered by the radiation emerging from a given volume element considered to be making up the contents of the box. Further, it is convenient, particularly in a tomography-like analysis scheme, to assume that the sample volume is divided into small volume elements (i.e. voxels). The voxel geometry, defined by the collimation, is illustrated below. The voxels are rectangular volumes with linear dimensions $\mathrm{a}, \mathrm{b}$ and $\mathrm{c}$ in the $\mathrm{x}-, \mathrm{y}-$, and $\mathrm{z}$-directions respectively. In practice, it is expected that the voxels will assume a cubic shape (i.e. $a, b$, and $c$ will be equal), but for generality that need not be the case. Each voxel is assumed to contain a uniform and homogeneous fill matrix defined, at each gamma-ray energy of interest, by the linear attenuation coefficient $\mu_{\mathrm{ijk}}$, and an activity $A_{i j k}, i, j$, and $k$ are the location indices of the voxel in the $x-, y-$, and $z$-directions respectively. Index $\mathrm{i}$ runs from 1 to $\mathrm{l}$, index $\mathrm{j}$ runs from 1 to $\mathrm{m}$ and index $\mathrm{k}$ runs from 1 to $\mathrm{n}$. Voxel $(1,1,1)$ is the front, bottom, left position shown in Figure 4 , while voxel $(1, m, n)$ is the back, top, right position. Even where the voxel paradigm is not used in the analysis, the labeling convention that falls out of this arrangement is still very useful in discussing the geometry and scanning protocols. 


\begin{tabular}{|l|l|r|}
\hline & Canberra Industries, ACK\# D0043 & 33296 B \\
\hline Created: 29-September-2000 & IBIS Project & Revision B \\
\hline Revised: 13-January-2003 & Preliminary Design Study & Page 27 of 156 \\
\hline
\end{tabular}

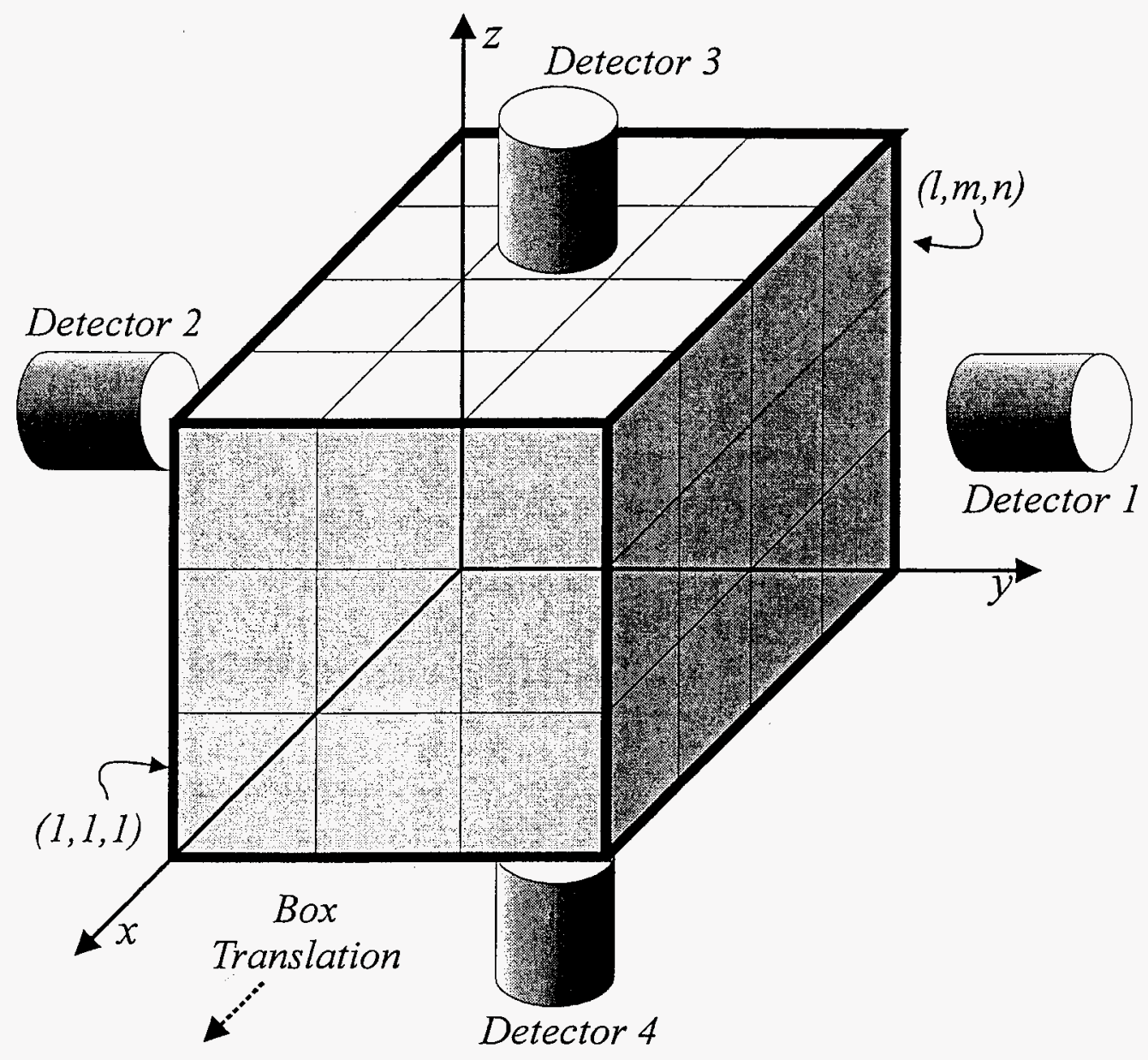

Figure 3. Schematic of Box Segmentation. The box moves along the $x$-direction. Detectors 1 and 2 scan vertically (z-direction) along the side boxes. Detectors 3 and 4 scan horizontally (ydirection) along the top and bottom of the box. 


\begin{tabular}{|l|l|r|}
\hline & Canberra Industries, ACK\# D0043 & 33296 B \\
\hline Created: 29-September-2000 & IBIS Project & Revision B \\
\hline Revised: 13-January-2003 & Preliminary Design Study & Page 28 of 156 \\
\hline
\end{tabular}

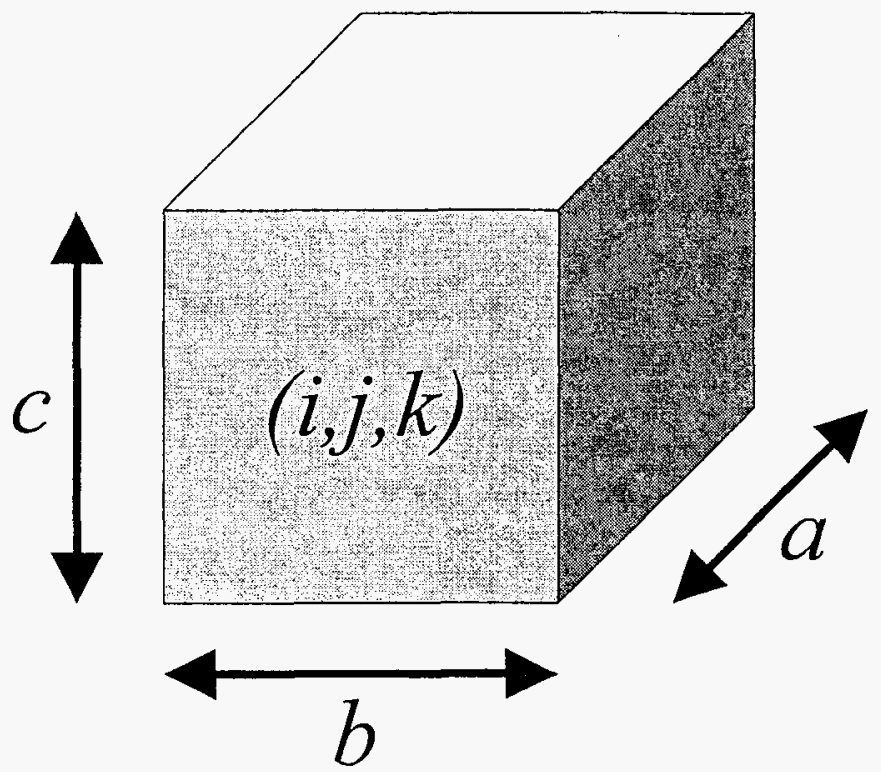

Figure 4. Single Voxel. The scanning protocol serves to effectively divide the box volume into small voxels having dimensions $\mathrm{a}, \mathrm{b}$, and $\mathrm{c}$ in the $\mathrm{x}, \mathrm{y}$, and $\mathrm{z}$ directions respectively.

The box enters the measurement station and the front segment $(i=1)$ is measured first with detectors $\mathrm{D}_{1}$ and $\mathrm{D}_{2}$ performing counts at stops corresponding to rows-of-sight $\mathrm{k}=1,2,3 \ldots \mathrm{n}$, and detectors $\mathrm{D}_{3}$ and $\mathrm{D}_{4}$ performing counts at stops corresponding to columns-of-sight $\mathrm{j}=1$ to $\mathrm{m}$. In this way each of the 1 - segments is measured by two detectors at n-stops and two detectors at $m$-stops. That is, $1 \bullet(2 m+2 n)$ spectra are recorded per box. Having two pairs of opposing detectors helps to even out the spatial response across the box and avoids having to turn the box in order to achieve the same effect. In addition to these emission spectra, each horizontal row-ofsight and vertical column-of-sight through each vertical i-segment of the box contents has its transmission factor measured by independent means. Thus there are $l \bullet(m+n)$ transmission factors available for use in any analysis.

The conceptual model set-up above involves $\mathrm{l} \bullet \mathrm{m} \bullet \mathrm{n}$ voxels, each requiring an activity and linear attenuation coefficient to specify it; that is, the model contains $2(1 \bullet m \bullet n)$ unknowns. The measurement ensemble provides $3(1 \bullet(m+n))$ pieces of information. For $m>3$ and $n>3$ arrangements, we can see immediately that the measurement is underdetermined. That is, too little information is available to solve for the contents (activity and attenuation) of all of the voxels unless other information, such as might be provided by multiple gamma-ray line analysis, is available. Thus, it is clear that some kind of numerical optimization scheme must be invoked. Furthermore, it is obvious that such a scheme will be quite complex because the problem is inherently non-linear. The problem is actually divided into two parts. The transmission data - the $1 \bullet(m+n)$ measured transmission values - can only be used to solve for the $1 \bullet m \bullet n$ unknown attenuation values. The passive emission data - the $2(l \bullet(m+n))$ measured emission rates for a given line - can be used to solve for both the $1 \bullet \mathrm{m} \bullet \mathrm{n}$ attenuation values and the $\mathrm{l} \bullet \mathrm{m} \bullet \mathrm{n}$ activities. This can be appreciated by noting that the measured responses depend on the 


\begin{tabular}{|l|l|r|}
\hline & Canberra Industries, ACK\# D0043 & 33296 B \\
\hline Created: 29-September-2000 & IBIS Project & Revision B \\
\hline Revised: 13-January-2003 & Preliminary Design Study & Page 29 of 156 \\
\hline
\end{tabular}

individual voxel contents modified by the attenuating properties of the intervening voxels which introduces products of terms.

To appreciate this further, it is instructive to develop the response function equations. It should be stated here that the goal is primarily to develop a good estimate of the total activity present in the box $\left(A=\sum_{i=1}^{l} \sum_{j=1}^{m} \sum_{k=1}^{n} A_{i j k}\right)$. The intent here is not necessarily to derive a tomography scheme; that is, it is not necessarily the intent to produce the complete image distribution of activity. For fissile materials this kind of information might best be provided by the neutron device which provides many more measured quantities.

\subsection{Gross Sensitivity of the BSGS System}

To estimate the sensitivity of the system, a series of MDA calculations was performed. The efficiency was obtained by modeling the system with Canberra's ISOCS software. The container was assumed to be a $54 \times 54 \times 88$ inch box, a corrugated metal container, the largest of the four most common container types expected. In the model, the detector was moved over the surfaces of the as in an actual assay - four positions along the width and height of the container and eight positions along the length, and thus 32 positions along each of the four long faces, which in total made 128 positions. At each position, the detector, a BE3825, was modeled at a distance of roughly $40 \mathrm{~cm}$ from the box surface, again mimicking an actual assay situation. Calculations were performed for several densities of the material in the box, which was assumed to be uniform. The acquisition times assumed 15 minutes per segment, which is equivalent to a total of 8 hours to count the entire box. Further, two source distributions were explored: a uniform activity distribution and a non-self-attenuating point source in the center of an otherwise inactive matrix. The background rates were obtained from empty-room measurements taken with a similar detector in a standard Box Counter shield. The Currie 95\% C.L. Critical Level form was used to estimate the detection limits

$$
1.6449 \sqrt{2 B T} /(\varepsilon T F)
$$

In this simple expression, $B$ is the background rate under the peak of interest, $T$ is the count time, $F$ is the gamma-ray branching ratio (i.e. gamma rays per second per decay), and $\varepsilon$ is the gammaray detection efficiency for the geometry [Currie 1968]. The following six nuclides and gamma rays were examined: 


\begin{tabular}{|l|l|r|}
\hline & Canberra Industries, ACK\# D0043 & 33296 B \\
\hline Created: 29-September-2000 & IBIS Project & Revision B \\
\hline Revised: 13-January-2003 & Preliminary Design Study & Page 30 of 156 \\
\hline
\end{tabular}

${ }^{239} \mathrm{Pu}: 414 \mathrm{keV}$

${ }^{238} \mathrm{U}: 1001 \mathrm{keV}$ (from the ${ }^{234} \mathrm{~Pa}$ daughter, assumed to be in equilibrium)

${ }^{241} \mathrm{Am}: 59.5 \mathrm{keV}$

${ }^{235} \mathrm{U}: 186 \mathrm{keV}$

${ }^{137} \mathrm{Cs}: 662 \mathrm{keV}$

${ }^{60} \mathrm{Co}: 1333 \mathrm{keV}$

The resulting datasets are depicted in the Figures 5 and 6.

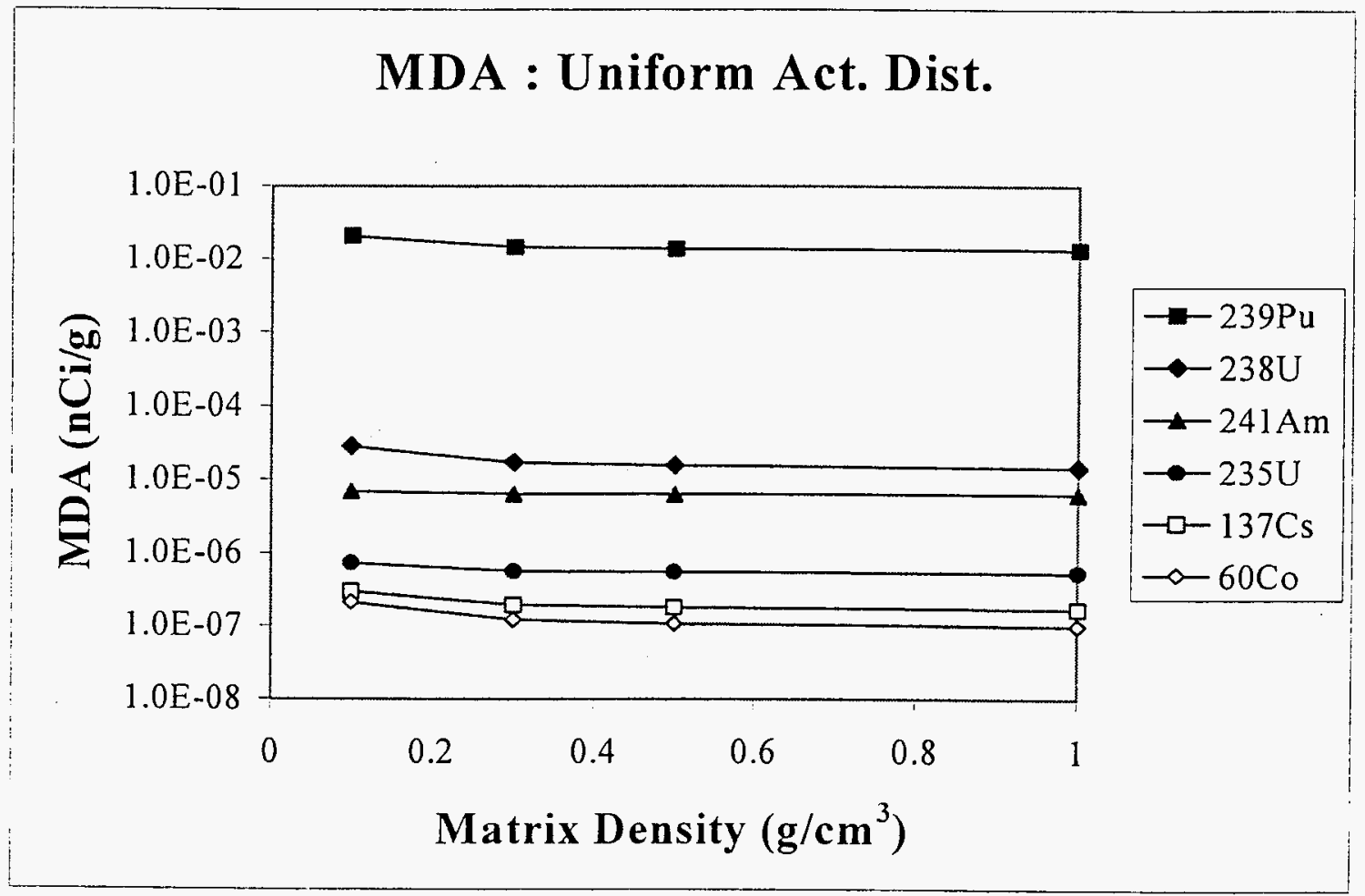

Figure 5. 


\begin{tabular}{|l|l|r|}
\hline & Canberra Industries, ACK\# D0043 & 33296 B \\
\hline Created: 29-September-2000 & IBIS Project & Revision B \\
\hline Revised: 13-January-2003 & Preliminary Design Study & Page 31 of 156 \\
\hline
\end{tabular}

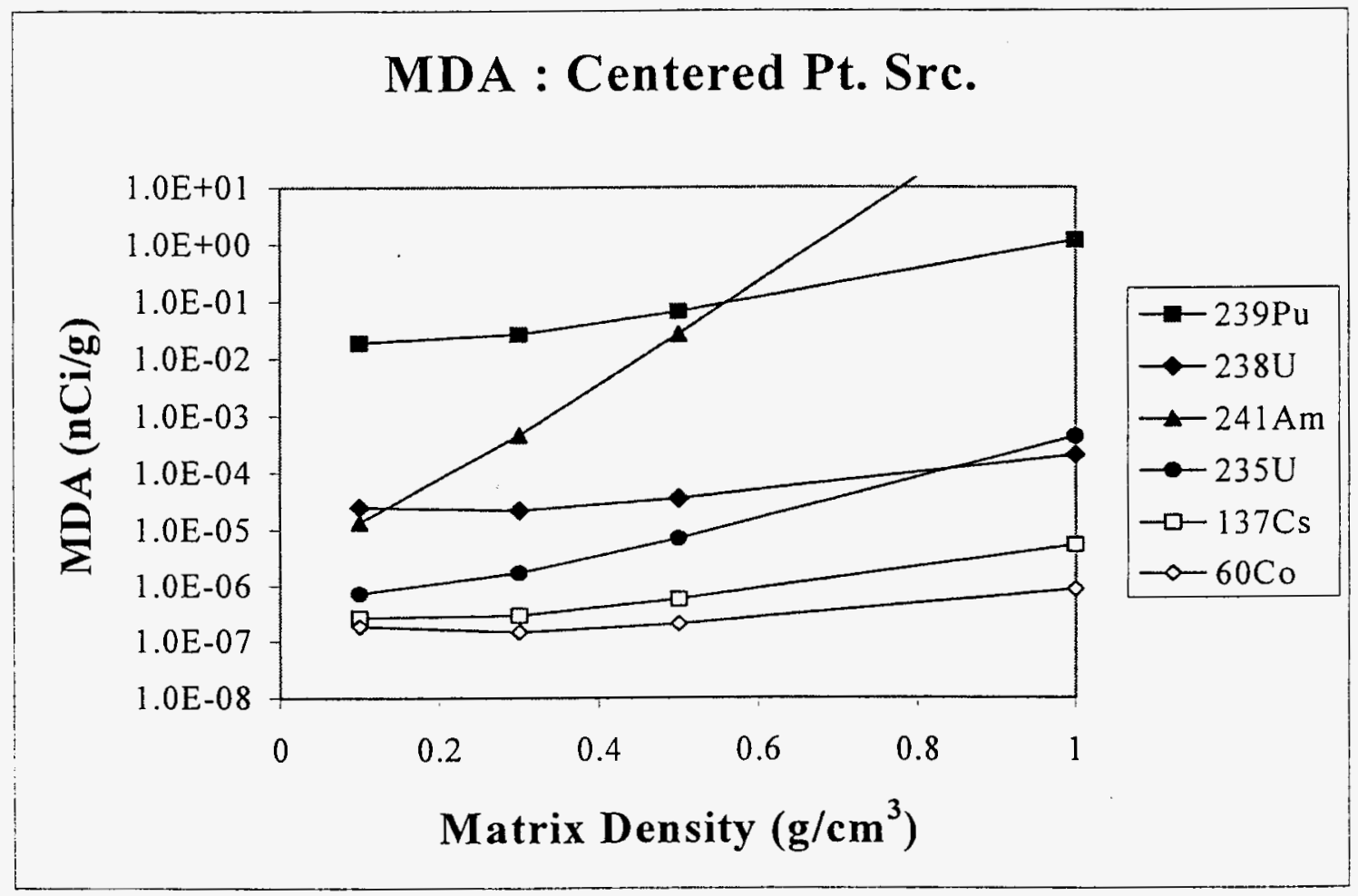

Figure 6.

It is evident that the MDA values for the uniform distribution are quite low, and, as expected, are dominated by the intrinsic detector efficiency shape and the emission intensity from each nuclide. The detection limits saturate very quickly with density, an effect which arises from the fact that, with a uniform distribution of activity, the material near the periphery of the container (i.e. the material actually "seen" by the detectors) is a good representative sample of the material throughout the box. For the point source data, the density plays a much larger role. Again this is as expected; with the single point source buried in the center of the box, the inactive matrix merely acts as a thick attenuator. Thus the detection limits rise with density, with the effect becoming more dramatic with lower energy gamma rays. Note that this data represents the sensitivity of the system to detect radioactivity and does not directly reflect the accuracy of the assay, with or without any non-uniformity correction scheme. The quantitative assay of ${ }^{241} \mathrm{Am}$ is problematic, especially if it is not collocated with $\mathrm{Pu}$, in large boxes because the $60 \mathrm{keV} \gamma$-lines is so weakly penetrating to the ${ }^{662} \mathrm{keV}$ line is of low abundance and can be masked by ${ }^{137} \mathrm{Cs}$.

\subsection{The Problems of Source and Matrix Non-uniformity}

Traditional gamma assay systems are usually implemented based on the assumption of a uniform spatial distribution of source activity and of matrix within the container. This is more than simply a matter of convenience during calibration. Consider a large population of containers, all with the 


\begin{tabular}{|l|l|r|}
\hline & Canberra Industries, ACK\# D0043 & 33296 B \\
\hline Created: 29-September-2000 & IBIS Project & Revision B \\
\hline Revised: 13-January-2003 & Preliminary Design Study & Page 32 of 156 \\
\hline
\end{tabular}

same size and material density, but with a random distribution of non-uniformities. Averaged over the entire population of containers, and ignoring more subtle effects such as the tendency for heavier materials and smaller particle sizes to settle to the bottom of a container, the best estimate of the distribution is uniform. Lacking any other information about an unknown container, one must assume that it is uniform. However, it is well known that any one container will almost certainly be non-uniform; this discrepancy between the single-case reality and the best overall assumption can lead to significant errors. Activity concentrated at the center of the container will generally be under-reported while activity on the periphery is over-reported. The effect is greater for denser matrices and for the lower gamma-ray lines. In extreme situations, non-uniform distributions of activity and of matrix density can easily exceed order of magnitude levels on the assay of individual items [Young, 1998]. NUCS is simple to implement and one would expect it to be incorporated on first generation systems even as more sophisticated methods are being developed.

At the simplest level, the spatial emission and transmission data provided by IBIS enables the validity of uniformity assumptions to be judged. By way of example, in the present arrangement it is a trivial problem to recognize the difference between a point source at the center of the box and a uniformly distributed activity. Such discrimination can be made by an intelligent data reviewer, or it can be coded into software. The next, and more desirable, level, then, is to utilize such a detailed dataset to attempt to correct for matrix and source inhomogeneities.

\subsection{Tomographic Analysis}

In simplest terms, tomographic analysis models the contents of the container as a collection of voxels and then attempts to solve for the attenuation (via the transmission and emission data) and the activity (via the emission data) associated with each voxel. In most gamma-ray emission scanning tomography, the information obtained from the measurement is in the form of $2 \mathrm{D}$ projections of the $3 \mathrm{D}$ radionuclide distribution; that is, in the form of images of selected object planes at desired orientations. The aim is to extract this $3 \mathrm{D}$ distribution by constructing a sequence of images of thin segments or slices. In the present context, an image can be thought of as a picture representing the spatial distribution of activity on an artificial scale of grayness or color. The aim is to obtain this sequence of information without distortion by attenuation and without cross-talk from neighboring slices. Such contributions would constitute artifacts, features in the image, which arise from errors other than those due to counting statistics. The discussion below describes the measurement and analysis framework behind a tomographic approach and attempts to declare the primary simplifying assumptions made.

As an intermediate step towards understanding the response of a system in a "voxel-ized" paradigm, the fraction, $f$, of signal emitted that emerges from a voxel can be estimated according to the uniform far field geometrical model

$$
f=\left(\frac{1-e^{-\mu t}}{\mu t}\right)
$$




\begin{tabular}{|l|l|r|}
\hline & Canberra Industries, ACK\# D0043 & 33296 B \\
\hline Created: 29-September-2000 & IBIS Project & Revision B \\
\hline Revised: 13-January-2003 & Preliminary Design Study & Page 33 of 156 \\
\hline
\end{tabular}

where $\mu$ is the linear attention coefficient of the medium in the voxel at the gamma-ray energy in question, and $t$ is the width of the voxel (either b or $\mathrm{c}$ ) in the direction of the passing beam. Written in terms of the transmission factor, $T$, across the individual voxel, the self-shielding factor can be expressed as

$$
f=\frac{1-T}{-\ln (T)}
$$

The transmission of an external interrogating source across the contents of the entire box is simply the product of all of the individual voxel transmission factors through which the interrogating beam passes.

Formulation of the problem is mathematically simplified by assuming that there is no cross-talk between single rows or columns. With a single tightly collimated transmission source, this is a valid assumption. Thus, each row and column can be analyzed independently and in the equations that follow the segment index ( $\mathrm{i}=1$ to $\mathrm{l}$ ) and gamma-ray energy index can be taken as implied. It is also necessary here to define the overall internal dimensions of the box in the $x_{-}, y_{-}$, and $\mathrm{z}$ - directions as $\mathrm{L}_{\mathrm{x}}(=\mathrm{l} \bullet \mathrm{a}), \mathrm{L}_{\mathrm{y}}(=\mathrm{m} \bullet \mathrm{b})$, and $\mathrm{L}_{\mathrm{z}}(=\mathrm{n} \bullet \mathrm{c})$, respectively.

Consider the transmission measurements across the contents of the box in horizontal direction $y$ at height stop $\mathrm{k}$. The transmission, denoted by TY(k), may written as

$$
T Y(k)=\prod_{j=1}^{m} T_{i j k}(b), \mathrm{k}=1 \text { to } \mathrm{n} \text { Eq. } 4
$$

where

$$
T_{i j k}(b)=e^{-\mu_{i j k} \bullet b}
$$

Similarly for the transmission measured in the vertical direction $\mathrm{z}$ at horizontal stop $\mathrm{j}$ the expression is

$$
T Z(j)=\prod_{k=1}^{n} T_{i j k}(c), \mathrm{j}=1 \text { to } \mathrm{m}
$$

where

$$
T_{i j k}(a)=e^{-\mu_{i j k} \bullet c}
$$




\begin{tabular}{|l|l|r|}
\hline & Canberra Industries, ACK\# D0043 & 33296 B \\
\hline Created: 29-September-2000 & IBIS Project & Revision B \\
\hline Revised: 13-January-2003 & Preliminary Design Study & Page 34 of 156 \\
\hline
\end{tabular}

To examine the passive emission data, consider that the contribution that each gamma-ray line, in each voxel, makes to the full energy peak count-rate in the passive emission spectrum of a given detector may be written as a product of:

A, the activity, (strictly the emission rate) of the line in that particular voxel.

$\varepsilon$ is the intrinsic full energy peak efficiency for gamma rays travelling towards the detector. For simplicity, bundled into this factor is any attenuation suffered by the gamma rays on their way to the detector face through the box wall, and any cadmium or tin low energy filters.

$G$ is a geometrical factor. To first order this is the inverse square solid angle factor between the sensitive volume of the detector and the face of the voxel closest to the detector.

$C$ is an empirical collimation factor which accounts for the fact that the collimator does not define an ideal columnar view across the box.

$F$ is the self-shielding of the activity provided by the material within the voxel itself.

On this basis the response (i.e. count rate) in detector $D_{1}$ when measuring segment $i$ at vertical stop $\mathrm{k}$ is given by

$$
D_{1}(i, k)=\sum_{j=1}^{m}\left(\varepsilon_{1} \cdot G_{1}(i, j, k) \cdot C_{1}(i, j, k) \bullet A_{i j k} \bullet f_{i j k}(b) \bullet \prod_{j=j+1}^{m} T_{i j k}(b)\right) \quad \text { Eq. } 8
$$

Similarly

$$
D_{2}(i, k)=\sum_{j=1}^{m}\left(\varepsilon_{2} \cdot G_{2}(i, j, k) \cdot C_{2}(i, j, k) \cdot A_{i j k} \bullet f_{i j k}(b) \bullet \prod_{j=1}^{j-1} T_{i j^{\prime} k}(b)\right)
$$

where by definition products involving terms outside the range, corresponding to the outermost cases, (e.g. $\prod_{j^{\prime}=1}^{0}$ and $\prod_{j^{\prime}=m_{+1}}^{m}$ ) are assigned values of unity. By extension the responses in the orthogonally disposed pair of detectors to $D_{1}$ and $D_{2}$ may be written as follows:

$$
\begin{array}{ll}
D_{3}(i, j) & =\sum_{k=1}^{n}\left(\varepsilon_{3} \bullet G_{3}(i, j, k) \bullet C_{3}(i, j, k) \bullet A_{i j k} \bullet f_{i j k}(c) \bullet \prod_{k^{\prime}=k+1}^{n} T_{i j k^{\prime}}(c)\right) \\
D_{4}(i, j) & =\sum_{k=1}^{n}\left(\varepsilon_{4} \bullet G_{4}(i, j, k) \bullet C_{4}(i, j, k) \bullet A_{i j k} \bullet f_{i j k}(c) \bullet \prod_{k^{\prime}=1}^{k-1} T_{i j k^{\prime}}(c)\right)
\end{array}
$$




\begin{tabular}{|l|l|r|}
\hline & Canberra Industries, ACK\# D0043 & 33296 B \\
\hline Created: 29-September-2000 & IBIS Project & Revision B \\
\hline Revised: 13-January-2003 & Preliminary Design Study & Page 35 of 156 \\
\hline
\end{tabular}

In these expressions for the responses

$$
f_{i j k}(b)=\left(\frac{1-e^{-\mu_{i j k} \bullet b}}{\mu_{i j k} \cdot b}\right)
$$

and

$$
f_{i j k}(c)=\left(\frac{1-e^{-\mu_{i j k} \bullet c}}{\mu_{i j k} \bullet c}\right)
$$

If the opposing pairs of detectors $\left(\mathrm{D}_{1}\right.$ and $\mathrm{D}_{2}$, and, $\mathrm{D}_{3}$ and $\left.\mathrm{D}_{4}\right)$ are nominally identical and similarly disposed then the corresponding $\mathrm{G}$ and $\mathrm{C}$ factors will be simply related by index switching to reflect the symmetry of the situation.

Despite the possible simplifications, this is clearly a non-trivial deconvolution analysis. It is one which is further complicated by the fact that the passive emission data have both the activity and the attenuation terms for the voxels folded into them, as indicated above. To implement this sort of approach with the BSGS system, the LANL NIS-6 TGS software can be modified to incorporate box geometries. It is currently designed to work only with cylindrical geometries. While the details of the TGS algorithm are beyond the scope of this document, the analysis technique does attempt a reconstruction of exactly the type discussed here. It is the intent of the measurement phase of this project to develop and test the concept of using this analysis engine for boxed waste.

\subsection{Canberra's NUCS}

Recently at Canberra, it was realized that a source-reconstruction algorithm would be a desirable add-on to existing drum-counting systems. A true tomography package, however, would have required extensive re-engineering of the standard acquisition and control software as well as the development of custom hardware to effect the scanning motion of the drum and detection equipment. Rather, it was desirable to have a simpler technique that would function within the bounds of existing segmented gamma-ray drum counters (e.g. SGS, IQ3) and existing control/acquisition/analysis packages (e.g. Genie-PC, GWAS). Towards this end the NonUniformity Correction Software (NUCS) package was developed. [Young, 1998].

Of vital importance to this package was the Dual Multi-Spectrum Scaler (DMSS) module used in the Canberra SGS and IQ3 systems, which allows for the collection of spectrum data as a function of time as the drum rotates. This provides the ability to divide the rotation period into eight equal portions, and thus collect spectrum data not only by vertical segment, but also by rotational "pie slice", or sector. For example, an IQ3 system has three vertically stacked detectors. With the DMSS module, each detector yields eight spectra, corresponding to eight rotational sectors. Thus, each gamma-ray line yields a total of 24 measured count rates or 


\begin{tabular}{|l|l|r|}
\hline & Canberra Industries, ACK\# D0043 & 33296 B \\
\hline Created: 29-September-2000 & IBIS Project & Revision B \\
\hline Revised: 13-January-2003 & Preliminary Design Study & Page 36 of 156 \\
\hline
\end{tabular}

"views". For any gamma-ray line, therefore, it is possible to study the variation in the line's intensity both vertically and axially. The NUCS algorithm was developed to utilize this data to correct for non-uniformities in the sample drum. This technique is not tomography; rather, it attempts to roughly localize the activities within the drum, and to calculate a correction factor to be applied to the normal assay value, which was obtained from the uniform assumption.

Consider a point source of activity $A$ embedded in a uniform matrix inside a drum and viewed by a pointlike detector outside the drum. The count rate $R$ in the detector from this point source is approximated by the expression

$$
R=\frac{A \varepsilon f(\Omega) e^{-\left(\frac{\mu}{\rho}\right) \rho d_{\text {marix }}}}{d_{\mathrm{sd}}^{2}}
$$

where

$d_{\mathrm{sd}}$ is the total distance between the source and the detector.

$d_{\text {matrix }}$ is the distance inside the matrix between the source and the detector.

$f$ represents the angular response of the detector (e.g. most detectors are more efficient onaxis than off-axis). If there is a collimator, that is also reflected in this function.

$\varepsilon$ is a constant that represents the intrinsic efficiency of the detector.

For a sample drum, the NUCS algorithm models the activity with five point sources and one uniformly-distributed activity. Each point source has four parameters: the source activity, and the three-dimensional position within the drum. Thus the entire model has 21 parameters. The distribution of activity within a real drum is approximated for each gamma-ray line by optimizing the model response, described in the expression above, against the set of segmented and sectored count rates for that line. Specifically, for a given segment and rotational segment, the expected count rate is calculated from the above expressions. This is then compared with the measured count rate at that position. A chi-squared value is obtained by summing over all of the segments and rotational positions. The model is optimized by varying the parameters (i.e. the activities and positions of the point sources and the activity of the uniform source) to minimize the value of chi-squared. Once the optimized parameters are obtained, the total model activity is compared against the calculated count rate for the uniform distribution to obtain a correction factor which is applied to the uniform assay result.

The model described above is highly complex, highly nonlinear, and subject to degeneracy (this is easily visualized by swapping the activities and positions of two point sources: physically the model is unchanged, but the list of parameters is different); therefore, an analytical approach to the minimization problem is not feasible. Instead, a hybrid technique is used which is a combination of the downhill simplex algorithm and a simulated annealing algorithm. The 


\begin{tabular}{|l|l|r|}
\hline & Canberra Industries, ACK\# D0043 & 33296 B \\
\hline Created: 29-September-2000 & IBIS Project & Revision B \\
\hline Revised: 13-January-2003 & Preliminary Design Study & Page 37 of 156 \\
\hline
\end{tabular}

objective behind this approach is to explore a large portion of the parameter space to find global minima without getting trapped in local valleys. While minimization techniques of this type are not applicable to all optimization problems and typically require fine-tuning for each application, the basic approach has been optimized and compared against experimental data collected by Canberra, and provides encouraging results.

The technique works well at higher densities and for tightly collimated geometries - those where the optimization model is most position-sensitive. For such situations, experimental data at Canberra has indicated that the algorithm reduces the measured discrepancy by typically a factor of two and drastically decreases the scatter (i.e. population standard deviation) in assay values of a large collection of non-uniform drums. The technique does not make significant improvements at low densities; this is still encouraging because at lower densities source non-uniformity does not contribute significantly to measurement discrepancies. Still, several improvements can be made to the algorithm to make it more robust for use with the BSGS.

The algorithm as it exists now is very closely wedded to cylindrical container shapes. A significant amount of re-engineering would be necessary to adapt the geometry routines in the software to work with box-shaped containers. There are certain symmetric geometries to which the algorithm is not sensitive. For example, a centered rod in a drum, a shell of activity on the periphery of a drum, and a uniform activity distribution in a drum all exhibit no significant variation in count rate as the drum rotates, and hence the NUCS algorithm cannot distinguish between them, even though all three will exhibit very different assay results. These sort of symmetric degeneracies are not possible with boxes, so it is expected that this weakness will not be a significant problem with the BSGS system. The algorithm is currently susceptible to poor statistics; it requires good data for each of the measured count rates it uses. Achievement of good counting statistics on all observation positions is not always feasible when counting boxes of material. This susceptibility can be removed by making the fitting model and the statistical assumptions built into it more robust. A specific mode of attack on this front is to revise the optimization target. Currently the software determines its activity model by minimizing the chisquared statistic, which is based on the assumption that the measured count rates have a Gaussian distribution about the "true" count rate. In practice, the Gaussian assumption is of questionable validity. Poor statistics and other non-statistical factors give rise to outlier points which can radically skew the results of a chi-squared minimization technique. A first step towards improving this situation is to replace the Gaussian assumption with the more realistic Poisson assumption, which is much more accurately forgiving in its acceptance of low-statistics outliers.

More significant revisions to be made concentrate on two points. First, the algorithm currently does not use multiple lines from a given nuclide. It optimizes each line individually. A more elegant approach is to recognize that multiple lines coming from the same nuclide would all have the same source distribution. This would drastically increase the amount of measurement data to bring to bear on the optimization problem. It is expected that this modification would significantly increase the robustness of the technique against low-statistics data. Second, the algorithm does not use any density profiling that may be obtained from transmission 


\begin{tabular}{|l|l|r|}
\hline & Canberra Industries, ACK\# D0043 & 33296 B \\
\hline Created: 29-September-2000 & IBIS Project & Revision B \\
\hline Revised: 13-January-2003 & Preliminary Design Study & Page 38 of 156 \\
\hline
\end{tabular}

measurements. NUCS currently assumes that the matrix is uniform and has a density specified by the user or obtained from the net weight of the sample container. While the source nonuniformity certainly makes a greater contribution to the overall measurement discrepancy, matrix inhomogeneity should not be ignored if the data are there to account for it.

\subsection{Lump Correction}

The above discussions on different techniques for non-uniformity correction concentrate largely on the error introduced to the assay results from attenuation in the sample matrix. This does not address self-attenuation in aggregate lumps of material. Typical techniques for accounting for this utilize a differential peak approach, where the measured intensity of two lines from the same nuclide are compared and used to derive a correction factor. This immediately poses a problem for nuclides such as ${ }^{235} \mathrm{U}$ which only has one strong gamma-ray line. These techniques rely heavily on assumptions about the shape of the lump (e.g. sphere's self-attenuate differently from cubes) and the material comprising the lump (e.g. the self-attenuation behavior of Pu metal is different from that of $\mathrm{PuO}_{2} \mathrm{~F}_{4}$ ). The most significant difficulty, however, is the fact that for larger lumps, where the self-attenuation error is the most severe, the differential peak techniques saturate. This is because once the gross dimensions of the lump become significantly larger than the attenuation length of the material, only the outside layer of the lump is "seen" by the detectors. As an extreme, but illustrative, example, consider a rod of Pu metal seen end-on. For the $375 \mathrm{keV}$ and $414 \mathrm{keV}$ lines, the ones usually used in a differential peak analysis of $\mathrm{Pu}$ bearing materials, the attenuation length is roughly $0.2 \mathrm{~cm}$. That means that if the rod is longer than roughly $0.6 \mathrm{~cm}$, no gamma-ray information from the far end of the rod is obtainable, and thus the technique cannot distinguish between a rod that is $0.8 \mathrm{~cm}$ long and $2 \mathrm{~cm}$ long. In short the correction technique breaks down for the lump sizes that need the most correction. Due to the state of the current and foreseeable technology as well as the limitations imposed by the physics, no attempt at lump correction will be made for the gamma-ray assay data. When multi-gram amounts of $\mathrm{Pu}$ are present it may be possible to exploit the $769 \mathrm{keV}$ line.

\subsection{Data Analysis}

Clearly, given the large number of segments in a given assay, both from the transmission scan and the passive emission scan, there are many possible analyses to which the data can be subjected. There are three main classes of data to be obtained from the BSGS assay. The first is a total activity value which has been corrected for source and matrix non-uniformity. This will be primarily useful for those nuclides to which neutron assay techniques are not sensitive, such as ${ }^{60} \mathrm{Co}$ and ${ }^{137} \mathrm{Cs}$. The issues involved and the techniques which can be brought to bear on this sort of problem have been discussed above. The second class of data to be obtained from the BSGS is plutonium and uranium isotopics. Towards this end, each of the segment passive emission spectra will be analyzed using Canberra's MGA and MGA-U software packages [Gunnik, 1990; Gunnik, et al., 1994]. These analysis packages utilize multiple lines from $\mathrm{Pu}$ and $\mathrm{U}$ to calculate the abundance ratios of the typical actinide isotopes. Current versions run in two modes. One mode concentrates on the lower energy lines, primarily below $110 \mathrm{keV}$ with the addition of one or two lines below $200 \mathrm{keV}$. The other mode utilizes higher energy (up to $700 \mathrm{keV}$ ) lines. This 


\begin{tabular}{|c|c|c|}
\hline & Canberra Industries, ACK\# D0043 & $33296 \mathrm{~B}$ \\
\hline Created: $29-\mathrm{S}$ & IBIS Project & Revision $\mathrm{B}$ \\
\hline Revised: 13-January-2003 & Preliminary Design Study & Page 39 of 156 \\
\hline
\end{tabular}

was primarily due to a limitation in the detector technology - the low energy mode was intended to work with Canberra's LEGe detectors, and the high energy mode was intended to work with standard coaxial detectors. With the recent availability of the BEGe detectors, which combine sensitivity at low energies and still maintain good resolution at high energies, it is possible to combine the two analysis modes to run on one spectrum. This change in the MGA and MGA-U packages will be implemented for use in the BSGS system. While the software does not determine the total activity or mass of the various isotopes, it has proven to be quite accurate in its isotopic calculations. The single-segment results can be combined to yield reliable isotopics data, which is a key element to obtaining meaningful results from a neutron assay. The third class of data is the density profile of the sample material obtained from the transmission assay. The density information is of primary importance for the accuracy of the gamma-ray assay, and a volume map of the material density will be constructed as part of the gamma-ray non-uniformity analysis. Neutron assay techniques are also susceptible to density effects. The gamma-ray analysis package will make its density map available to the neutron algorithms. The neutron tomography technique proposed for the BSNS will be able to distinguish and correct for regions of different material composition in the sample on a gross scale. The accuracy of this volume reconstruction is expected to be greatly enhanced by prior knowledge of the density profile of the material in the container.

\subsection{Calibration}

Of concern for a system with many measurement segments and with very large sample types are the various calibrations necessary for an accurate assay. It is desirable for the system to be easy, both in terms of time and expense, to calibrate. For the BSGS, with only four detectors, energy calibration is straightforward. The same is true of the transmission calibration - the unattenuated (a.k.a. empty-container) count rates from the transmission sources into their associated detectors. The most difficult calibration for the BSGS will be the efficiency calibration. Calibration by direct measurement of known (e.g. NIST-traceable) standards that mimic the waste stream is infeasible here - given the possible combinations of box size and matrix density, the sheer numbers become untenable, without even considering the problems of source procurement and storage. Clearly an alternative approach is necessary to simplify the process and reduce costs. More recently, with the increasing speed and availability of computers, mathematical calibrations have been gaining acceptance as a solution for situations of this sort. The BSGS will have its calibrations performed using Canberra's ISOCS software [Bronson, Young, Atrashkevich, 1997; Bronson and Young, 1997]. This software package calculates efficiency curves for nearly any practical geometry. The user interface allows the operator to first select from a menu of basic geometry templates (e.g. a box, or a cylinder). Next, the operator specifies the details of this template, such as the dimensions and wall thickness of the sample container and the material composition, density, and fill height of the material in the container. After then specifying the energies at which to calculate the efficiency points, the operator starts the calculations. At this stage, the software package is, at its core, a numerical integration routine; it divides the active sample volume into voxels. The voxels are treated as point sources, and the software calculates the contribution to the total efficiency from each voxel, accounting for the attenuation from each voxel through other materials in the geometry. To obtain the single-voxel point source efficiency, 


\begin{tabular}{|l|l|r|}
\hline & Canberra Industries, ACK\# D0043 & 33296 B \\
\hline Created: 29-September-2000 & IBIS Project & Revision B \\
\hline Revised: 13-January-2003 & Preliminary Design Study & Page 40 of 156 \\
\hline
\end{tabular}

a numerical model of the detector response is developed at the Canberra factory from actual measurements made with the detector and a set of NIST-traceable sources.

Calculations for a large, multi-segmented, collimated geometry such as the BSGS typically take approximately 4 days, and the accuracy for these geometries has been found, from extensive testing and validation, to be typically within $30 \%$. Thus, the ISOCS solution offers a much more desirable calibration scenario than the procurement of numerous surrogate source containers. Confidence in the ISOC-calculated efficiencies will be established by benchmarking the results to direct measurements performed on the set of surrogate containers (obtained from TMFA) that will be used to establish the response characteristics of the neutron system. These measurements will be conducting using a combination of radionuclide standards as well as $\mathrm{U}$ and Pu sources. 


\begin{tabular}{|l|l|r|}
\hline & Canberra Industries, ACK\# D0043 & 33296 B \\
\hline Created: 29-September-2000 & IBIS Project & Revision B \\
\hline Revised: 13-January-2003 & Preliminary Design Study & Page 41 of 156 \\
\hline
\end{tabular}

\section{Differential Die-Away Analysis}

In the late 1970 s, Caldwell and others applied pulsed neutron concepts to the assay of ${ }^{239} \mathrm{Pu}$ and ${ }^{235} \mathrm{U}$ in 208-liter waste drums. By measuring prompt neutrons from the thermal interrogation region, they were able to achieve sensitivity levels better than $10-\mathrm{nCi} / \mathrm{g}$ in a counting time of 40 seconds [Kunz, et al., 1980]. The new technique for waste assay was called the differential dieaway technique (DDT). This name refers to the relative neutron lifetimes or dieaway times between the interrogating thermal flux in the assay chamber and fast neutrons in the detection packages [Caldwell, et al., 1986]. Over the years, the specifics of the technique and instrument designs have changed, but the assay of waste by prompt neutron detection using pulsed neutron methods is still referred to as DDT. In this section we briefly review the history of pulsed neutron methods and indicate why DDT is the method of choice for the next generation prototype box counter.

During the past 50 years, pulsed neutron experiments have been used to study neutron transport in materials of interest to the nuclear community. Experiments have been performed on multiplying and non-multiplying materials with analytical techniques varying in complexity depending on the physical properties being studied. A typical experiment consists of injecting short bursts of high-energy neutrons at fixed intervals into a medium and recording the time behavior of the neutron intensity. As a neutron interacts with nuclei of the system, it loses energy through inelastic and elastic collisions until it reaches thermal energies. Once thermal, neutrons diffuse and eventually decay with an average lifetime that is to first order a function of the absorption and leakage properties of the medium.

For an approximately 15-year time span beginning in the mid 1950s, pulsed neutron techniques were used extensively to measure basic neutronic parameters such as diffusion coefficients, absorption cross sections, differential scattering cross sections, and neutron lifetimes. Since experimentally measured parameters are averaged over the neutron distribution within the medium, correct interpretation of the data relies on theoretical studies to determine the distribution as a function of space, energy, and time. Thus, pulsed neutron research during this era not only included the measurement of these parameters but also the development of new approximations to the analytical and numerical solutions of the time-dependent transport and diffusion equations.

Research in pulsed source techniques prior to 1965 was motivated by the needs of reactor designers. However, the technique's sensitivity to both the properties of the medium and the presence of fissile material led to applications in other fields such as the quantitative assay of ${ }^{239} \mathrm{Pu}$ and ${ }^{235} \mathrm{U}$ in nuclear safeguards and uranium ore exploration in geophysics. An early example of nondestructive determination of fissile material is the adaptation of the lead slowingdown-time-spectrometer (LSDTS) [Bergman, et al., 1955] to the measurement of irradiated and non-irradiated fuel elements. [Stegemann and Seufert, 1968; Krinninger, Wiesner, and Faber 1969; Sawan and Conn, 1974; Block, et al., 1989; and Abdurrahman, 1995; Armitage, 1989]. 


\begin{tabular}{|l|l|r|}
\hline & Canberra Industries, ACK\# D0043 & 33296 B \\
\hline Created: 29-September-2000 & IBIS Project & Revision B \\
\hline Revised: 13-January-2003 & Preliminary Design Study & Page 42 of 156 \\
\hline
\end{tabular}

The slowing down assay time method is an active, nondestructive method based on the slowing down of neutrons from a pulsed source centered in a lead slowing-down-time spectrometer. The injection of a fast neutron pulse into the lead results in a neutron population with a mean energy inversely proportional to the square of the slowing down time. Fast neutron detectors located close to the fissile material are used to detect the induced fission neutrons. The selection of these detectors is based on their ability to measure fast neutrons while remaining insensitive to epithermal and thermal neutrons. Thus various fast detector options include fission chambers, gamma-shielded liquid and plastic scintillation counters, hydrogen and methane proportional counters, cadmium-wrapped, polyethylene-moderated ${ }^{3} \mathrm{He}$ or $\mathrm{BF}_{3}$ proportional counters or ${ }^{4} \mathrm{He}$ proportional counters.

The main advantage of the LSDTS is its sensitivity to fissile material while maintaining a high signal to background ratio. The technique also offers, in principle, the ability to co-assay ${ }^{235} \mathrm{U}$ and ${ }^{239} \mathrm{Pu}$ by measuring fission neutrons during separate time intervals that correspond to energy ranges where large differences exist in their fission cross sections. However, the presence of the large moderating lead assembly makes the LSDTS practical only for geometrically small samples.

By contrast the pulsed neutron logging of uranium boreholes by the prompt fission neutron technique, which was already well established in the mid 1970s [SAND77-0300], is an example of a compact probe (or logging tool) that is intended for insertion into a large body of uranium bearing ore. From this perspective, it is the direct opposite of the LSDTS, i.e., a detector inside the sample. However, the fundamental principles of the measurements are similar, resting on earlier theoretical investigations [Koppel, 1960; Zakharchenko, 1962; Czubek, 1972]. The general method is as follows:

A short burst of $14 \mathrm{MeV}$ neutrons is generated losing energy in the surrounding rock formation and thus creating a thermal flux that rises, peaks and then decays. If uranium is present in the formation, the thermal flux induces fission in ${ }^{235} \mathrm{U}$ producing prompt fast neutrons that are detected by a high-pressure, polyethylene-moderated ${ }^{3} \mathrm{He}$ proportional detector wrapped in cadmium. The cadmium, which is essentially black to thermal neutrons, prevents detection of the interrogating flux and thereby provides the necessary discrimination to allow induced fission neutrons to be detected with good signal to background ratio. In addition to the prompt neutrons, delayed neutrons are ejected from the fission fragments. The intensity of delayed neutron emissions is a small fraction $(\sim 1 \%)$ of the prompt signal and is spread out in time governed by the half-lives of the delayed neutron precursors (up to $\sim 56 \mathrm{~s}$ ). However, these delayed neutrons can be counted in the period between $14 \mathrm{MeV}$ neutron bursts as an alternative signal, albeit with a lower sensitivity and lower duty cycle background suppression factor. In this case, the signal is the result of the fission's induced over the whole lifetime of the interrogating flux, i.e., the fast, epithermal and thermal energies all contribute.

Having originally been developed for uranium borehole logging, but with clear theoretical associations with the LSDTS, the differential dieaway technique became a tool that was also 


\begin{tabular}{|l|l|r|}
\hline & Canberra Industries, ACK\# D0043 & 33296 B \\
\hline Created: 29-September-2000 & IBIS Project & Revision B \\
\hline Revised: 13-January-2003 & Preliminary Design Study & Page 43 of 156 \\
\hline
\end{tabular}

applied to the problems of spent reactor fuel [Rotter, 1974], TRU-waste assay [Kunz, et al., 1980; Kunz, et al., 1981] and the detection of special nuclear material diversion [Smith and Rice, 1981; Lillie, et al., 1981]. Since these first field-worthy DDT systems appeared, the technique has been adopted and further developed by a number of groups around the world. Interest in the technique remains high today and the method continues to evolve. As in its inception, the fundamental appeal of the DDT method remains its high sensitivity and specificity to the fissile isotopes. These attributes allow rapid assays of lightly contaminated wastes for screening purposes, or alternatively, enable the technique to be applied to hot wastes with high neutron backgrounds such as head end debris [Hampson, 1993].

Due to high sensitivity and duty cycle suppression of the background, the minimum detectable mass in a DDT assay is less affected by neutron background than the other widely known active neutron technique, the ${ }^{252} \mathrm{Cf}$ shuffler. DDT is also insensitive to the levels of $(\alpha, n)$ and curium contamination that would render passive neutron time correlation analyses unused. A general discussion of the capability and limitations of a general purpose 208-liter drum-sized DDT system may be found elsewhere [Nicholas, et al., 1992; Rinard, 1994]. High performance DDT systems can also offer a reasonably good passive neutron detection capability integrated into the same instrument. For plutonium assay, this allows the low mass range to be addressed using the active interrogation element and the higher mass range (when self-shielding of the interrogating flux may be present) to be addressed using the passive mode. This dual modality gives complete and seamless coverage and enables the rapid screening of LLW as well as the quantification of TRU waste to be performed in a single device.

Early, dedicated, DDT systems had quite modest detection efficiencies of only a few percent. Some modern designs have efficiencies in the teens of percents. This confers quite a good passive neutron counting capability, especially if all the efficiency is derived from fast neutron detector packages. The cadmium-wrapped detector packages have an inherently shorter dieaway time than bare ${ }^{3} \mathrm{He}$ proportional detectors embedded in the chamber wall minimizing the problem of accidental coincidences. Furthermore, because the detector packages are decoupled from the thermal neutrons in the assay chamber, the coincidence gate utilization factor is less variable from matrix to matrix and the spatial variation of the coincidence response throughout a container remains flatter. As a consequence, the response variation from matrix to matrix tracks the square of the total efficiency (in coincidence mode) much more closely, simplifying matrix correction formulations and resulting in a more accurate compensation. Thus general-purpose combined active-passive instruments have evolved that can address a broader range of assay needs. The active component provides a total fissile mass determination while the passive mode provides ${ }^{240} \mathrm{Pu}_{\text {eff }}$ quantification [Coop, Nov. 1989]. 


\begin{tabular}{|l|l|r|}
\hline & Canberra Industries, ACK\# D0043 & 33296 B \\
\hline Created: 29-September-2000 & IBIS Project & Revision B \\
\hline Revised: 13-January-2003 & Preliminary Design Study . & Page 44 of 156 \\
\hline
\end{tabular}

Other major developments over the years have been:

- Exploitation of the asymmetric geometry technique [Swinhoe, 1986; Packer and Swinhoe, 1988; Haruyama, et al., 1999] to achieve a flatter radial response profile in difficult-to-assay wastes by physically arranging the efficiency and interrogating flux profiles to approximately compensate. This technique relies to some extent on the self-generation of the thermal interrogating flux within the matrix itself.

- Attempts to correct for the self absorption effects observed in lumps of fissile material by using time windows corresponding to neutron energies that are more penetrating [Coop, June 1989; Coop and Hollas, 1996] or by using the delayed neutron signature [Armitage and Cogbill, 1989].

- Innovative step of placing the pulsed neutron generator in a separate moderating assembly or side optimization block [Caldwell, et al., 1992; Lucero and Caldwell, 1996; Chard, 1991] outside the cavity. Thus a persistent plane wave of mixed epithermal and thermal neutrons could be directed into the chamber in a way that de-emphasized the spectrum tailoring and flux maintaining properties of the interior cavity walls. With a reduced chamber wall thickness, higher detection efficiency could be achieved. By surrounding the neutron generator with a $(n, 2 n)$ target, the fast neutron yield was increased resulting in a higher thermal interrogating flux intensity. This combined effect on the efficiency-flux product resulted in an overall improvement in sensitivity, albeit with a somewhat poorer global matrix penetrability due to the softer injected spectrum.

- Exploration of the role of active coincidence counting [Caldwell, et al., 1992; Chard and Croft, Sept. 1998] as a means of:

a. Co assaying ${ }^{235} \mathrm{U}$ and ${ }^{239} \mathrm{Pu}$;

b. Generating inherent matrix transmission (detection efficiency) corrections;

c. Improving the signal-to-background ratio (even though the absolute specific signal is lower, the absolute coincidence background rate is also much lower);

d. Acquiring imaging information with better contrast. Monte Carlo simulation subroutines that allow the $n-n$ angular and energy correlations to be modeled to track localized coincidence behavior are only now beginning to emerge. [Lestone, 1999].

- Concerted efforts have been made to develop, understand and demonstrate realistic and complete total measurement uncertainty propagation procedures [Harker, et al., 1995; Blackwood, et al, 1999]. These efforts tackle the fundamental question of the confidence level a facility manager should place on the assay results depending on the end use of the data. It also helps directly identify areas for improvement. 


\begin{tabular}{|l|l|r|}
\hline & Canberra Industries, ACK\# D0043 & 33296 B \\
\hline Created: 29-September-2000 & IBIS Project & Revision B \\
\hline Revised: 13-January-2003 & Preliminary Design Study & Page 45 of 156 \\
\hline
\end{tabular}

- Application of empirical data-driven methods of data treatment as an alternative to the model-driven algorithmic paradigm that is the current norm [Becker, et al., 1995]. Such methods encompass the various architectures of artificial neural networks, fuzzy logic methods, genetic evolution theory and combinations of these and other techniques for analyzing and classifying nonlinear multivariate problems such as fractal, chaos, expert systems and formal statistical techniques. The assay result from a DDT instrument is a highly intertwined relationship of many physical variables (efficiency profile, flux profile, source distribution, matrix characteristics etc) that in turn affect the observed count-rates in the various detector banks and matrix monitors in quite a complex fashion. This makes selection, optimization and quantification of the performance improvement of traditional model-driven algorithmic data analysis schemes non-trivial. Fortunately, DDT is also potentially data rich provided the instrument has been designed with this in mind. The combination of a rich data stream and hidden complexity is exactly the kind of environment one might anticipate empirical computational tools, which can self-organize and adapt to realize arbitrary nonlinear mathematical dependencies to produce good results. The appreciation of these methods, which is only just beginning in the field of NDA waste assay, will be assisted by instrumentation designed to generate the matrix and response signals needed to capture the underlying physical complexity.

- Exploitation of list mode data acquisition and analysis nucleonics that brings great flexibility and offers new and exciting ways of extracting information from the pulse streams available [Brunson and Arnone, 1997; and later discussion].

- Introduction of source imaging techniques in an analysis-based effort to reduce the assay bias caused by the non-uniform spatial distribution of fissile material within the waste [Estep, Melton, and Miko, 1998].

- Development of comprehensive matrix compensation schemes using flux monitors and flux monitors in conjunction with the familiar Californium add-a-source approach used in passive counting [Chard, Croft, and Sharp, 2000 and references therein; Melton, Estep, and Hollas, 1998].

- Implementation of ways to scan the neutron generator (to supplement sample rotation, physical asymmetric compensation and imaging corrections) in order to achieve a more uniform interrogation [Melton, Estep, and Peterson, 2000].

These developments and the subsequent refinements that can be envisioned, given improvement in computing power, suggest that DDT has the potential to become an even more versatile and effective waste assay technology provided intellectual resources can be brought to bear on the key issues. To date, the most complete expression of the technology base outlined above available in a field-ready version is the CTEN (Combined Thermal/Epithermal Neutron) system developed by the Los Alamos National Laboratory for the measurement of 208-liter drums. This system is described in a US DOE document [DOE/EM-0465] and references therein. The innovations embodied in this device and the possibilities outlined above have yet to be 


\begin{tabular}{|l|l|r|}
\hline & Canberra Industries, ACK\# D0043 & 33296 B \\
\hline Created: 29-September-2000 & IBIS Project & Revision B \\
\hline Revised: 13-January-2003 & Preliminary Design Study & Page 46 of 156 \\
\hline
\end{tabular}

implemented fully in a larger scale counter suitable for the measurement of boxed waste [Becker, McIlwain, and Connolly, 1999].

The CWAM (Crated Waste Assay Monitor) [Melton, Estep, and Peterson 2000] developed for the measurement of uranium-contaminated boxed waste at the Y-12 plant in Oak Ridge is clearly illuminating the way to the next generation of such counters. In order to facilitate this next step, a prototype crate counter configured to allow flexible data acquisition sufficient to demonstrate the performance ability of all currently conceived data reduction schemes is needed. In addition to pushing back the frontiers of individual present generation boxed waste measurement instruments (e.g. DDT, PNCC, passive gamma) such an instrument would allow for the first time a systematic, experimentally founded study of how to integrate data from different subsystems in order to obtain a better assay result. For example, the image of fissile material generated from the active DDT interrogation could well provide a valuable overlay to improve the accuracy of the passive neutron count. Similarly it is conceivable that $\gamma$-ray transmission factor data could be used to modulate active assay matrix correction matrix elements improving the quality of the DDT images. It was as a result of such a vision as this that the IBIS project was born. 


\begin{tabular}{|l|l|r|}
\hline & Canberra Industries, ACK\# D0043 & 33296 B \\
\hline Created: $29-$ September-2000 & IBIS Project & Revision B \\
\hline Revised: 13-January-2003 & Preliminary Design Study & Page 47 of 156 \\
\hline
\end{tabular}

\section{Box Segmented Neutron Scanner}

The BSNS incorporates much of the technology from the LANL CTEN and CWAM projects. Methods of data acquisition and analysis developed for CTEN have improved assay accuracy and sensitivity for drum-sized containers. Sets of cavity flux and matrix monitors have been included to determine matrix effects, due to moderation and absorption so that corrections can be made to the measured results. The position dependent information is an integral part of this analysis. The neutron generator will be mounted on a motorized track to allow interrogation of a waste crate from several positions inside the chamber. The ability to move the generator provides a more uniform interrogation of the waste crate and allows derivation of positional information through source position scanning and heterogamous matrix assessment.

The current CWAM is designed to measure B-25 boxes with dimensions $4^{\prime} \times 4^{\prime} \times 6^{\prime}$. For BSNS the measurement chamber will be enlarged to accommodate containers measuring up to $5^{\prime} \times 5^{\prime} \mathrm{x}$ $8^{\prime}$ with the detector and monitor layouts re-optimized to accommodate $>95 \%$ of DOE waste forms. The design calculations presented here are indicative and it should be noted that box counters of the type discussed can be size scaled over quite a wide dynamic range.

\subsection{Counter Design}

The modularity of the design is an important factor in allowing the construction of an optimally designed system early in the second year of the project, leaving time for a significant measurement campaign. This permits construction of certain key components, such as the fast neutron detector packages, to take place at the same time that MCNPTM Monte Carlo [Briesmeister, 1997] studies are being performed to resolve the final details of "neutronic" design questions. Modularity is also seen as key to creating a system which can be transported with reasonable ease. A major design objective is to reduce the weight of the BSNS to allow it to be trailer mounted.

\subsection{List Mode Data Acquisition}

Neutron coincidence and/or multiplicity techniques are the basis for most passive neutron assays. Until recently most neutron NDA systems relied on the shift register to sort detector-pulses into timing histograms. One negative aspect of this type of pulse processing by hardware, is that the original data stream is lost. A module developed at Los Alamos several years ago, known as the PATRM [Arnone, Brunson, and Coop, 1992], allows the original time history of pulses to be recorded, allowing different software algorithms to process data. Other types of data manipulation and analysis, such as the unambiguous rejection of cosmic-ray background neutrons, determination of dead time characteristics, and the detection of double pulsing and noise bursts can also be performed straightforwardly. The PATRM is currently used in waste assay systems such as CTEN and CWAM. A new PCI-PATRM card has been under development and will be completed in time for the BSNS to take advantage of the associated enhancement in data accumulation options especially in active mode. 


\begin{tabular}{|l|l|r|}
\hline & Canberra Industries, ACK\# D0043 & 33296 B \\
\hline Created: 29-September-2000 & IBIS Project & Revision B \\
\hline Revised: 13-January-2003 & Preliminary Design Study & Page 48 of 156 \\
\hline
\end{tabular}

Traditional neutron data acquisition devices; such as multiplicity shift registers (MSR), essentially collapse the incoming raw time history data into just a few characteristic values, such as Totals and Reals (or coincidence) count rates, by hardware implementation of a data analysis algorithm. This compression of the pulse arrival time history into other data domains is often acceptable for data streams that are essentially static (that is time invariant as in the case of a passive neutron count or periodic as in the case of an active neutron assay) or when the arrival rate of events is too high by other means. However, in this approach, having mined the ore which is the data stream for nuggets of information that the algorithm can sift out, the original pulse arrival time history is lost, being discarded as waste just as tailings are discarded from an actual ore shifting process.

An alternative approach is to sequentially record and store into memory the arrival time (relative to some initiating signal) of neutron events from every channel of data. This is called list mode data acquisition (LMDA). In principle a high speed time and frequency LMDA instrument (one that can handle the incoming data rate; time stamp the events with a resolution that is small compared to the characteristic transients of the physical systems under study; and in doing so introduces negligible instrumental artifacts such as dead-time biases) records all that can ever be known from a given set of data streams. Appropriate LMDA instrumentation in conjunction with accompanying software modules to process the data list are a very versatile and flexible tool for addressing not only routine measurement problems but also experimental investigation of new situations and concepts.

Any desired data treatment can be implemented in software and alternative analyses can be applied to exactly the same sequence of data. Indeed, the parameters used in the analysis don't even have to be selected until after the data has been acquired. Thus in addition to being able to implement different algorithms without the overhead of having to have different electronic hardware equipment, the data can be re-analyzed to check the sensitivity of the results to various parameter settings For example, these parameter settings in the MSR could be clock frequency, pre-delay, and the coincidence gate width. Various data filtering techniques such as noise identification and rejection ca also be easily implemented.

The utility of LMDA has long been established and several high performance units are commercially available; some of these measure the interval between successive pulses rather than the time from some initiating event, but the principle is the same [Guide Technology Incorporated, 1998; Computer I/O Corp, 2000]. In addition to arrival time, the sequence of data recorded can also, in principle, be tagged with other information such as the data stream of origin and any other associated quantity that may be of interest such as temperature, amplitude, etc. For neutron counting applications the list need only store arrival time and channel identification.

A CAMAC based Pulse Arrival-Time Recording Module (PATRM) developed by Arnone [Arnone, Brunson, and Coop, 1992]. has been successfully applied since the early 1990s for both passive neutron correlation counting [Brunson and Arnone, 1997] and for active neutron pulse train analysis [Hollas, et al., 1996; Hollas et al., 1997]. In active neutron interrogation, such as 


\begin{tabular}{|l|l|r|}
\hline & Canberra Industries, ACK\# D0043 & 33296 B \\
\hline Created: 29-September-2000 & IBIS Project & Revision B \\
\hline Revised: 13-January-2003 & Preliminary Design Study & Page 49 of 156 \\
\hline
\end{tabular}

differential die-away analysis (DDA), the interrogating flux is periodically applied to the sample. The signal must be collected as a function of time after injection of the interrogating neutrons. The time trace provides important diagnostic information about the way the neutrons interact with and connect to the various materials and regions of the assay cavity and the sample inside it. In basic DDA systems, the temporal information is extracted using multiple scalers, each gated on for a specific time window following the interrogation burst. In more sophisticated systems each channel of interest is fed into a multichannel scaler system to generate a distribution of events in time. The distribution is generally built-up by summing the counts collected from one sweep to the next.

Multichannel scaling allows the impact of different timing gate boundaries to be investigated and also allows curve-fitting techniques to be applied. Generally the approach is fine for data channels that provide information with no inherent correlation between pairs of detected events. Examples are the external matrix monitors and cavity flux monitors used to derived normalization and matrix correction factors. However, the fissile signal data streams do contain correlation's arising from the detection of two or more neutrons originating from a common induced fission event. In collapsing the incoming data streams into a MCS domain the correlation information is lost. An interrogation triggered LMDA module provides a convenient means of capturing the full information content (although other ways of performing limited correlation analysis are possible [Ma, et al., 1994; Chard and Croft 1999] with special arrangements for treating accidental coincidences).

There are many benefits for adoption of LMDA into a waste assay instrumentation research and development program such as this. They include:

- A convenient way of summing multiple input channels without introducing additional deadtime losses.

- Recording of the whole neutron pulse train offers access, via software engines, to all available analysis techniques [Reilly, et al., 1991]. These include all variants of auto correlation analysis on summed pulse trains including: Rossi - $\alpha$ pulse interval analysis; event triggered shift register analysis including coincidence and multiplicity analysis to all orders; variable dead time counter analysis; Feynman variance analysis [Brunson, 1983; Pázit and Yamane, 1998; Pál, 1958]; periodic (random) and event triggered time correlation analysis; squaring and cubing circuitry simulation. 


\begin{tabular}{|l|l|r|}
\hline & Canberra Industries, ACK\# D0043 & 33296 B \\
\hline Created: 29-September-2000 & IBIS Project & Revision B \\
\hline Revised: 13-January-2003 & Preliminary Design Study & Page 50 of 156 \\
\hline
\end{tabular}

- The stored data can be retrieved and reanalyzed using alternative algorithms or analysis parameters. One has the freedom to collect the data and then choose the parameter setting needed in the analysis afterwards. For example, the clock-rate predelay and coincidence gate width that are usually fixed at the time of data collection when using shift register circuitry can be treated as variables in the analysis of list mode data.

- Exactly the same data stream can be replayed through different analysis engines to test for biases between different methods.

- The pulse streams can be compared directly to analog Monte-Carlo simulations [Bruggeman, et al., 1996]. One can envision using statistical methods to do this, especially in cases where the point model commonly used to interpret time correlation count fails. This allows, for example, prompt and albedo (reflect or delayed) multiplication fission train transients to be explored in greater detail. Treated this way multiplicity histograms can be analyzed directly using Bayesian statistical inference to compare the calculated and experimental distributions. This avoids the usual subtraction process needed to treat the accidental coincidence events. The subtraction process often is precision limiting.

- High multiplicity cosmic-ray background events can be recognized and rejected by various schemes including individual channel data filters, channel-to-channel data filters, and summed channel data filters.

- Electronic noise bursts and other spurious signals can be filtered with increased clarity and their influence on the assay result quantified by carrying out a sensitivity analysis. In general, noise events do not share the characteristic die-away profile of genuine neutron events.

- The correct operation of LMDA modules may be easily tested using pulse train generators.

- The dead-time of individual channels and of the system can be assessed directly from the auto correlation plot measured using a random neutron source. The interpulse probability plot allows the dead-time to be monitored during an actual data run. Innovative dead-time correction approaches suggest themselves, such as the idea of scanning the data list and inserting, in a random but realistic way, the lost pulses. Such an approach, in principle, is not limited to the conventional dead-time model assumptions. It can for example attempt to include an allowance for the rate dependent increased probability of detecting a second event at early times after the nominal dead period. This arises due to the possibility of an event that would normally be just below threshold being detected if it sits on the transient (tail) caused by the pulse preceding it.

- Analysis of the interpulse probability plot allows direct diagnosis of the system for anomalies such as double pulsing. 


\begin{tabular}{|l|l|r|}
\hline & Canberra Industries, ACK\# D0043 & 33296 B \\
\hline Created: 29-September-2000 & IBIS Project & Revision B \\
\hline Revised: 13-January-2003 & Preliminary Design Study & Page 51 of 156 \\
\hline
\end{tabular}

- The on-line processing of list mode data (to produce for example 2-D Rossi- $\alpha$ plots) allows real time graphical visualization of time correlation spectra and multi dimensional surface analysis and data cuts. These are useful when setting up the instrument and for monitoring routine performance.

- LMDA data can be readily configured into conventional single pass, variable channel width, multi channel scaler (MCS) histogram format in software to permit differential die-away analysis of transients over extended periods. In DDA systems this allows coverage from temporal regions where neutronically interconnected regions give rise to diagnostic transients, out to time regions where delayed neutron events are registered.

- Cross correlation analysis between the different input detector channels, or groupings of channels, suggests itself as a means of both filtering background events and also of performing imaging of fissile material. This is possible in both passive and active assay modes.

- LMDA provides a convenient tool for acquiring the data needed to perform time dependent multiplicity correlation analysis during pulsed neutron interrogation.

For the reasons set out above we intend to incorporate into IBIS a significantly enhanced version of Amone's PATRM unit. The new unit is designated PATRM/PCI to denote the conversion from CAMAC to PC card format and the adoption of the Peripheral Component Interconnect Version 2.2 bus architecture. PCI is a high performance expansion bus architecture developed by Intel and now implemented as an industry standard. The conceptual design specification of the PATRM/PCI module has been laid down by Arnone [Arnone, 2000]. The development of PATRM/PCI addresses not only the user requirement for a less costly and more compact alternative to the CAMAC board PATRM unit, but also the requirement for larger high speed static memory arrays to extend the dynamic range of the instrument (both means rates and stream lengths) and finer time resolution, so that the accuracy of the analysis is not limited by the coarseness of the arrival time record.

Some of the improvements of the PATRM/PCI module over its predecessor are:

1. Input channel ID tagging. Each module will be able to accept 32 independent input channels.

2. Programmable channel selection and routing.

3. Programmable channel delay gating. 


\begin{tabular}{|l|l|r|}
\hline & Canberra Industries, ACK\# D0043 & 33296 B \\
\hline Created: 29-September-2000 & IBIS Project & Revision B \\
\hline Revised: 13-January-2003 & Preliminary Design Study & Page 52 of 156 \\
\hline
\end{tabular}

4. Finer time resolution. Each event will be recorded as a 64 bit word. The first 32 bits represents the bit pattern of the 32 input channels; the second 32 bits represents the number of ticks of the internal $100 \mathrm{MHz}$ clock since the initiating signal. In this way 32 simultaneous events can be accepted, channel and time stamped, and recorded without additional dead-time losses being incurred. The 32 bit deep counter allows $2^{32}$ ticks of the clock to be represented. Each tick is spaced by $10 \mathrm{~ns}$ (the reciprocal of $100 \mathrm{MHz}$ ) and so a data stream can extend to $\left(2^{32} \times 10 \times 10^{-9}\right) 42.9 \mathrm{sec}$, with each event recorded over this duration, being time stamped with a resolution of $10 \mathrm{~ns}$. When the clock rolls over, a marker word is written to the data file so that the occurrence can be easily recognized and handled by the analysis engine.

Each channel can handle an instantaneous burst of data up to $50 \mathrm{MHz}$ for $9 \mu \mathrm{s}$.

5. In-line data analysis using programmable firmware for near real time reporting of key measurements parameters. This approach is flexible. It allows different cuts of the data to be taken as required by using a reprogramming tool rather than complete re-engineering / replacement of the hardware. For sustained routine, high rate runs this approach allows data reduction to be carried out on the fly thereby avoiding the need to store and analyze large lists off-line.

6. Virtual data storage to handle higher rates and larger volumes of incoming data. By using a sequential (FIFO) buffering technique from very fast PATRM/PCI memory to CPU board memory to local disk, higher transfer rates can be sustained, the size of the data array can be enlarged and the cost per input channel can be reduced. In essence the fast on-board memory temporarily stores the incoming data which is transferred to the PC memory at the rate the PCI bus can handle it.

7. A lower cost and more convenient form factor. The module will be configured as a PC card.

8. Two or more synchronized boards allow more channels to be covered.

9. The module is intended to be used in pulsed neutron generator applications. For example initiation of the timing sweep can be taken directly from the neutron generator trigger.

10. The system will incorporate more sophisticated internal data checks to monitor, for example, "bit bobbles", and to deal with scaler rollovers.

The PATRM/PCI module provides a high capability, high functional density package that is not available from currently available commercial vendors. It has also been specified with DDA applications in mind. These are principal reasons for selecting it over other commercially available units.

Ways of improving tomographic imaging will also be explored. This is an important new application of the PCI-PATRM that was not possible with the previous version. The 


\begin{tabular}{|l|l|r|}
\hline & Canberra Industries, ACK\# D0043 & 33296 B \\
\hline Created: 29-September-2000 & IBIS Project & Revision B \\
\hline Revised: 13-January-2003 & Preliminary Design Study & Page 53 of 156 \\
\hline
\end{tabular}

PATRM/PCI provides a very natural way to reconfigure the detection geometry in relation to the pulsed neutron source in order to explore "asymmetric" arrangements for which the interrogating flux profile and the detection efficiency profile combine to form a product that has a reasonably flat spatial dependence across the box.

Although the absence of a rotational component will make image reconstruction more limited in crate systems than in drum systems, the post analysis of pulse trains from different combinations of tagged channels will enable localized coincidence counting to be examined as an alternative. In principle this will allow sharper imaging than is achievable using singles neutron counting. In general, to support this approach, the detector packages must crisscross the crate in a way that gives both vertical and horizontal positional information. Additionally the algorithms used in the CTEN analysis will require modification to accommodate the rectangular geometry and data richness.

Gauging the fill level of the matrix in the crate will be addressed using the grid of small fast (epi$\mathrm{Cd}$ ) and thermal (bare) ${ }^{3} \mathrm{He}$ filled neutron detectors inside the door. These detectors enable the moderation and absorption properties of the matrix to be estimated. The magnitude and temporal profile of these monitors form the basis of the matrix compensation. It is currently envisioned that six pairs of matrix monitors (each pair is a fast-thermal couple) be arranged in two vertical columns of three. Each monitor will have its own preamplifier and will feed into a separate channel of a PATRM/PCI module. The array of matrix monitors is intended to provide spatial information about the neutronic properties of the matrix.

\subsection{Advanced Passive Neutron Assay Analysis}

Canberra has implemented a suite of algorithms for the reduction of background rates, improvements in assay bias and precision. These algorithms have been tested on 200 liter drum samples but have not yet been applied to the assay of large waste boxes or crates. The implementation of these techniques can result in a factor of 10 reduction in assay bias for low mass $\mathrm{Pu}$ assay and a factor of 2 reduction in the assay precision. The recently developed algorithms that have resulted in these improvements are:

1. Add-A-Source Background Correction for matrix moderation effects by adaptation of the Add-A-Source Technique for correction of the fissile signal strength.

2. High-Z Background Correction: Separation of the cosmic-ray induced neutron signal from the high- $Z$ components of the sample matrix, from the neutron signal from the fissile content of the matrix. The assay of a sample containing high- $Z$ materials such as steel or lead may otherwise result in a positive bias. 


\begin{tabular}{|l|l|r|}
\hline & Canberra Industries, ACK\# D0043 & 33296 B \\
\hline Created: 29-September-2000 & IBIS Project & Revision B \\
\hline Revised: 13-January-2003 & Preliminary Design Study & Page 54 of 156 \\
\hline
\end{tabular}

\section{a. Known Matrix Method}

If the high- $Z$ component of the sample matrix is known by either process knowledge or by Real Time Radiographic examination (RTR) it is possible to adjust the measured neutron rates by an empirically determined correction factor.

\section{b. Multiplicity Difference Method}

This algorithm allows direct measurement of the matrix induced background increase using multiplicity analysis. The characteristic relative proportions of the singles, doubles, and triples neutron coincidence rates from the cosmic-ray induced background are different from those obtained from measurement of Pu standards. These differences can be exploited to remove the cosmic-ray induced neutrons by solving a simple set of linear equations.

3. Truncated Multiplicity applied to waste assay or low fissile mass measurements provides precision enhancements using a variable endpoint truncation of the measured multiplicity histogram to reduce the cosmic-ray components of the background correction and matrix induced interference.

This technique also allows improved performance of the above techniques (items 1 and 2) in addition to previously impractical analysis techniques such as the "known multiplication" method for multiplicity analysis.

4. Automated Algorithm Selection to facilitate the appropriate choice of analysis technique has been developed but has not yet been implemented into Canberra's normally available software.

The application of these algorithms will enable the BSNS to provide assays on some matrices with too high a moderator content for the active neutron interrogation to be successful. They are expected to be especially useful for matrices containing large quantities of moderator and lead or cadmium.

\subsection{Integration of the Add-A-Source (AAS) Correction Technique}

A scanning AAS system will be integrated into the BSNS for use with the passive and active neutron assays. The AAS technique has been found to be very accurate for matrix correction of homogeneous source distributions within moderately heterogeneous matrices. The scanning AAS mechanism can also provide information on the distribution of matrix materials within the sample [Armitage B.H., et al., 1995]. The addition of the AAS will be studied to determine if it is advantageous for improved active neutron assay.

\subsection{Physical Form of Radioactive Material}

The BSNS provides both passive and active neutron measurement of the sample. Self-shielding by lumps in active neutron mode present an insurmountable problem experimentally. Source 


|
\begin{tabular}{|l|l|r|}
\hline & Canberra Industries, ACK\# D0043 & 33296 B \\
\hline Created: 29-September-2000 & IBIS Project & Revision B \\
\hline Revised: 13-January-2003 & Preliminary Design Study & Page 55 of 156 \\
\hline
\end{tabular}

tailoring (injection of a hard flux) is not expected to help penetrability of lumps because of the rapid thermalization caused by the moderator in the boxes. In passive mode a positive bias can result when multiplication effects can not be corrected. Multiplication correction is generally not possible in waste assay when the chemical form of the plutonium is not well known and the matrix moderation introduces large errors into the correction calculation. In some situations, multiplicity analysis can provide additional information that can be used to flag this condition.

\subsection{Calibration Concerns}

The BSNS system is based on a new technology and will require initial testing over a large number of sample matrices. Due to the increase in the type and quantity of data obtained for each sample compared to the standard CTEN/CWAM type systems it is expected that the number of matrix types will be reduced for routine calibration purposes based on the results of the initial testing. Also an extensive library of Monte Carlo calculations will be built up in the design phase and benchmarked by the measurements. These will provide a data base for interpolation and extrapolation with respect to the measured matrix set. 


\begin{tabular}{|l|l|r|}
\hline & Canberra Industries, ACK\# D0043 & $33296 \mathrm{~B}$ \\
\hline Created: 29-September-2000 & IBIS Project & Revision B \\
\hline Revised: 13-January-2003 & Preliminary Design Study & Page 62 of 156 \\
\hline
\end{tabular}

There are clear cost savings in the rectangular FNDP module approach in preference to the cylindrical, "individual detector" FNDP's, due to the considerably simpler machining requirements. A rectangular FNDP package can be constructed using two "back to back" HDPE slabs with rectangular slots cut in them, to allow insertion of the counters. A quick calculation shows that for a fixed number of ${ }^{3} \mathrm{He}$ counters, there would be a greater cadmium mass overall (some $31 \%$ ) if they were configured as individual cylindrical FNDP's. Consider for example, a 4 - detector FNDP pack, with a fixed detector length $x(\mathrm{~mm})$. The surface area of Cadmium would be $2 x(303+80)=766 x \mathrm{~mm}^{2}$. Four separate cylindrical packages of the same length would have a total surface area of $4 x . \pi(80)=1005 x \mathrm{~mm}^{2}$. This means that the cosmic ray induced background will be lower, and also the DDA flux poisoning would be slightly less pronounced, for the rectangular package approach. It is considered that there would be little difference in detection efficiency (for a given total number of counters) between the two approaches, and so the cost savings afforded by the rectangular modular FNDP approach mean that this is considered to be the best option.

The FNDP design used for the present chamber design calculations, comprises 2 inch $\mathrm{OD}^{3} \mathrm{He}$ counters, of 4 atm. fill pressure, embedded centrally (and equally spaced) in an HDPE block of width $296.8 \mathrm{~mm}$ and depth $71.8 \mathrm{~mm}$. The block is clad externally with $1.6 \mathrm{~mm}$ thick cadmium sheet. The active length of the counters has been taken to be $1360 \mathrm{~mm}$.

\subsubsection{Possible Alternatives to Cadmium}

The use of cadmium in the FNDP's to shield the detectors from the interrogating thermal neutron flux, acts as a target for cosmic ray spallation events, such that the neutron background is elevated. This is a well documented effect, and is most serious for heavy "high- $Z$ " metallic elements. The use of an alternative material has been suggested. Boron has a similar thermal neutron cross - section to cadmium, and has a " $1 / \mathrm{v}$ " cross - section profile which may be useful in absorbing a significant fraction of the epithermal flux in addition to the thermal flux. Borated rubbers and plastics can be obtained, but in order to obtain a similar macroscopic cross - section to the $1.6 \mathrm{~mm}$ thick cadmium, the dimensions of these materials become prohibitively large, such that they begin to interfere with the neutronic behavior of the assay system. On the other hand, natural gadolinium has a microscopic cross - section for low energy neutrons which is a factor of $\approx 20$ higher than for cadmium. This means that to achieve a given attenuation factor for a fixed cadmium thickness, a much thinner layer of a "Gd - rich material" is needed. This would give benefit in that the cosmic ray induced neutron background would be much lower due to the lower target mass. As cosmic - ray generated spallation neutrons in the chamber body represent a significant component of the passive neutron Totals and Reals background, such a saving is worthwhile investigating. In addition, gadolinium has a somewhat different neutron energy dependence than that of $\mathrm{Cd}$, and this may also have benefit. 


\begin{tabular}{|l|l|r|}
\hline & Canberra Industries, ACK\# D0043 & 33296 B \\
\hline Created: 29-September-2000 & IBIS Project & Revision B \\
\hline Revised: 13-January-2003 & Preliminary Design Study & Page 63 of 156 \\
\hline
\end{tabular}

It is known from experimental experience, that the FNDP background count rate is very sensitive to the integrity of the cadmium liner. Increasing the Cd thickness from 1 to $2 \mathrm{~mm}$ reduces the active background by approximately $30 \%$. The seating of the cadmium end cap on the top of the FNDP was found to be very important, as is the crimping of the cadmium funnel over the signal cable. Failure to take practical measures to ensure a good coverage of cadmium was found to lead to factors of approximately 3 increase in the background. Indeed, there may be a good argument for packing the end caps with Boron or Lithium loaded putty to minimize the thermal neutron leakage into the detectors. It is possible that by painting a $\mathrm{Gd}$ rich compound directly onto the surfaces, these leakage problems may be reduced. Other practical issues which would need consideration, are the availability and cost of gadolinium compounds, their chemical toxicity, and hence any special safety / handling precautions which may be necessary.

Cadmium can be readily obtained in metallic form (density $8.65 \mathrm{~g} . \mathrm{cm}^{-3}$ ). Gadolinium could be readily obtained in the form of gadolinium oxysulphide $\left(\mathrm{Gd}_{2} \mathrm{O}_{2} \mathrm{~S}\right.$, density $\left.=7.44 \mathrm{~g} . \mathrm{cm}^{-3}\right)$, and this is thought to be a possibility for use in a "paint compound" which colild be coated on to the outside of the detector packages. Natural gadolinium comprises 14.8 weight $\%{ }^{155} \mathrm{Gd}$ and 15.7 weight $\%{ }^{157} \mathrm{Gd}$. These are the only isotopes which need be considered in assessing the neutron cross-section, as they dominate the capture cross-section of the natural element.

The variation of microscopic cross-section with energy is shown in Figure 7, for both natural cadmium and natural gadolinium. At thermal energies the microscopic cross-section for natural $\mathrm{Gd}$ is a factor of $\approx 20$ greater than that for $\mathrm{Cd}$. At $\mathrm{keV}-\mathrm{MeV}$ energies, the cross-sections are comparable. In the intermediate $(1-100 \mathrm{eV})$ region, the resonance cross-sections for $\mathrm{Gd}$ are considerably higher than for Cd. However, in the region of the cadmium cut-off $(\approx 0.1-0.5 \mathrm{eV})$, the gadolinium cross-section is up to a factor of a few lower than for cadmium. To assess the relative performance of gadolinium and cadmium as shields against the interrogating neutron flux, it is necessary to fold together the energy profiles for the ${ }^{3} \mathrm{He}(n, p)$ reaction rate, the gadolinium or cadmium $(n, \gamma)$ capture cross-section, and the interrogating flux, $\varphi$, over a typical DDA counting interval. To this end, the energy distribution of the DDA interrogating flux, integrated over a time region of $(0.8-10 \mathrm{~ms})$, and averaged over the volume of an empty box, has been used. An MCNPTM model with $150 \mathrm{~mm}$ thick graphite, and an FNDP embedding depth of $44 \mathrm{~mm}$, has been used. The background count rate in the detectors, $\mathrm{c}$, is proportional to the ${ }^{3} \mathrm{He}(\mathrm{n}, \mathrm{p})$ reaction rate, which is given by Equation 15 , where $\Sigma$ is the macroscopic absorption cross-section for either cadmium or gadolinium, $t$ is the thickness of the cadmium or gadolinium layer, and $\sigma_{\mathrm{He}}$ is the ${ }^{3} \mathrm{He}(\mathrm{n}, \mathrm{p})$ microscopic cross-section. 


\begin{tabular}{|l|l|r|}
\hline & Canberra Industries, ACK\# D0043 & 33296 B \\
\hline Created: 29-September-2000 & IBIS Project & Revision B \\
\hline Revised: 13-January-2003 & Preliminary Design Study & Page 64 of 156 \\
\hline
\end{tabular}

The energy variation of the macroscopic cross-sections for $\mathrm{Cd}$ and $\mathrm{Gd}$, in the forms of metal and oxysulphide, as described above, are shown together with the DDA interrogating flux spectrum, in Figure 8.

$$
c \propto \int_{E=0}^{\infty} e^{-\Sigma(E) \cdot t} \cdot \sigma_{H e}(E) \cdot \phi(E) \cdot d E
$$

The energy variation of the FNDP background estimate, given by the quantity c, as obtained from Equation 15, is shown graphically in Figure 9. The effect of the sharp resonance in the Cd crosssection at $0.2 \mathrm{eV}$ (see Figure 7) is evident as a depression in the contribution to c. The value of $\mathrm{c}$, integrated over all energies, is illustrated in Figure 10, normalized to the default case of $1.6 \mathrm{~mm}$ thick cadmium. The results show that one would require a layer of the Gd compound whose thickness is a factor of approximately 4 lower than for cadmium metal.

\subsection{MCNP ${ }^{\text {TM }}$ Modeling results}

\subsubsection{Calculation of Number of FNDP's}

Using the base model comprising $150 \mathrm{~mm}$ thick graphite walls, with an outer layer of $100 \mathrm{~mm}$ HDPE and an assumed FNDP embedding depth of $14 \mathrm{~mm}$, a detection efficiency of $\approx 22 \%$ was obtained for a total of 30 FNDP's each containing $4{ }^{3} \mathrm{He}$ counters. This assumed embedding depth was consistent with a previous, Harwell DDA $200 l$ drum chamber design. There were 6 FNDP's in each of the Front, Back, Top and Bottom walls, with 3 FNDP's in each of the RHS and LHS walls, making a total of $120^{3} \mathrm{He}$ counters. The embedding depth, $\mathrm{d}$, is defined by the distance from the inner graphite surface, to the front surface of the rectangular FNDP (see Figure 11).

\subsubsection{FNDP Die-Away Time}

The calculated FNDP die-away profile following a $14 \mathrm{MeV}$ burst is illustrated in Figures 12,13 and 14, for the case of an empty box, with an FNDP embedding depth of $14 \mathrm{~mm}$. Over the time range $0-100 \mu$ s the profile is a good fit to a single exponential (Figure 12), with a die-away time $(\tau)$ of $\approx 32 \mu$ s. Plotted over the range $0-500 \mu$ s, there is some evidence of an additional, long lived component. Fitted over the range $0-200 \mu$ s, the single exponential fit changes to $\approx 39 \mu \mathrm{s}$. This is due to fast neutrons which penetrate the outer cadmium of the FNDP's, and are then held up in time due to scattering in the HDPE inside the FNDP's. 


\begin{tabular}{|c|c|c|c|}
\hline \multirow{3}{*}{ in } & & Canberra Industries, ACK\# D0043 & $33296 \mathrm{~B}$ \\
\hline & Created: 29 -September-2000 & IBIS Project & Revision $\mathrm{B}$ \\
\hline & Revised: 13-January-2003 & Preliminary Design Study & Page 65 of 156 \\
\hline
\end{tabular}

A calculation of the die-away time based on diffusion theory, is presented in Appendix A. Using these methods, the die-away time has been estimated to be $\approx 22 \mu$ s for an FNDP design of the type considered here (taking a value of $t=12.5 \mathrm{~mm}$, in Table 15 of Appendix A), comprising four S/S walled ${ }^{3} \mathrm{He}$ counters of $50.8 \mathrm{~mm}$ OD and 4 atmospheres fill pressure. This shows fair agreement with the values given above, calculated by MCNPTM.

It is interesting to note, however, that the die-away time calculated by diffusion theory is significantly shorter than from the MCNPrM modeling. It is expected that one likely cause of this discrepancy is the leakage of epithermal neutrons from the surrounding graphite and HDPE, through the cadmium and into the FNDP moderator. The diffusion theory model takes the FNDP in isolation; the outer cadmium is assumed to decouple the FNDP from its neutronic environment so that the surrounding HDPE and graphite need not be considered. As the absorption crosssection in graphite is low, the in-scatter of epithermal neutrons from the graphite into the FNDP's, will introduce a long - lived component into the die-away profile observed inside the FNDP's, thereby lengthening the die-away time. An additional contributory factor to the difference between diffusion theory and MCNPTM, is expected to be the homogenization of FNDP elemental components in the former.

Appendix A gives a discussion on the benefits associated with a short FNDP die-away time, in the context of DDA counting. This method is very useful in that it facilitates a systematic analytical study of the effects of the various FBDP parameters, on the die-away time. This approach has the advantage of not having to perform a separate, time - consuming MCNP ${ }^{\mathrm{TM}}$ run for each case. 


\begin{tabular}{|l|l|r|}
\hline & Canberra Industries, ACK\# D0043 & 33296 B \\
\hline Created: 29-September-2000 & IBIS Project & Revision B \\
\hline Revised: 13-January-2003 & Preliminary Design Study & Page 66 of 156 \\
\hline
\end{tabular}

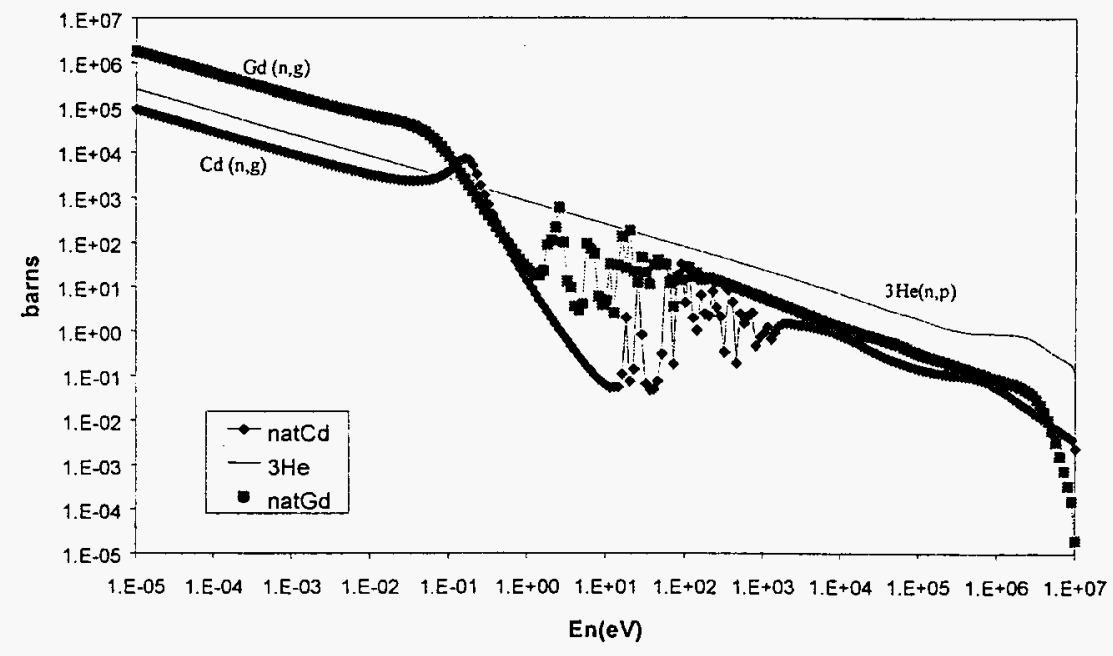

Figure 7. Microscopic $(\mathrm{n}, \gamma)$ capture cross-section for cadmium and gadolinium, shown together with the cross-section profile for the ${ }^{3} \mathrm{He}(\mathrm{n}, \mathrm{p})$ reaction.

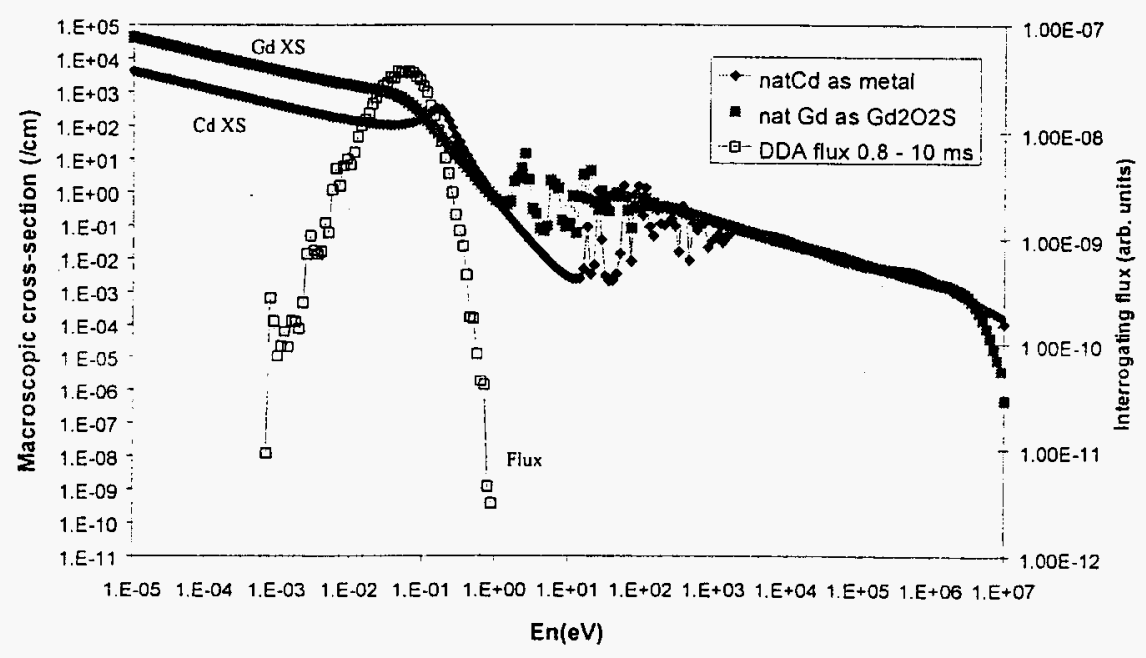

Figure 8. Macroscopic cross-sections for $\mathrm{Cd}$ and $\mathrm{Gd}$, shown with the DDA interrogating flux spectrum. 


\begin{tabular}{|c|c|c|c|}
\hline \multirow{3}{*}{ m } & & Canberra Industries, ACK\# D0043 & $33296 \mathrm{~B}$ \\
\hline & Created: 29 -September-2000 & IBIS Project & Revision $\mathrm{B}$ \\
\hline & Revised: 13-January-2003 & Preliminary Design Study & Page 67 of 156 \\
\hline
\end{tabular}

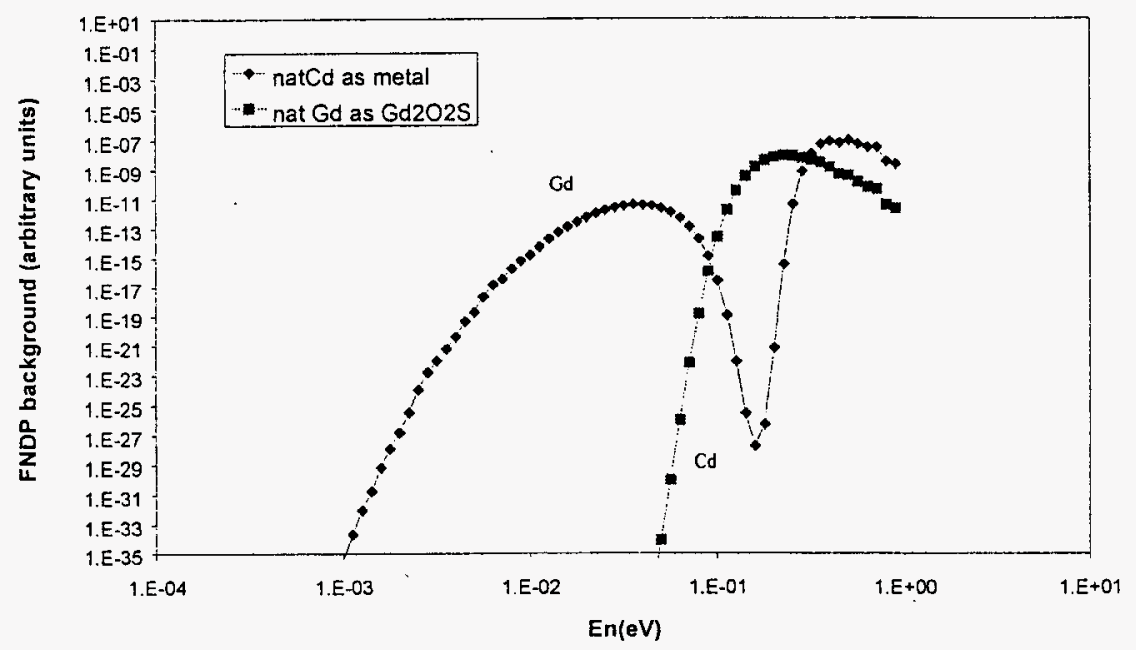

Figure 9. Predicted contribution to FNDP background as a function of energy.

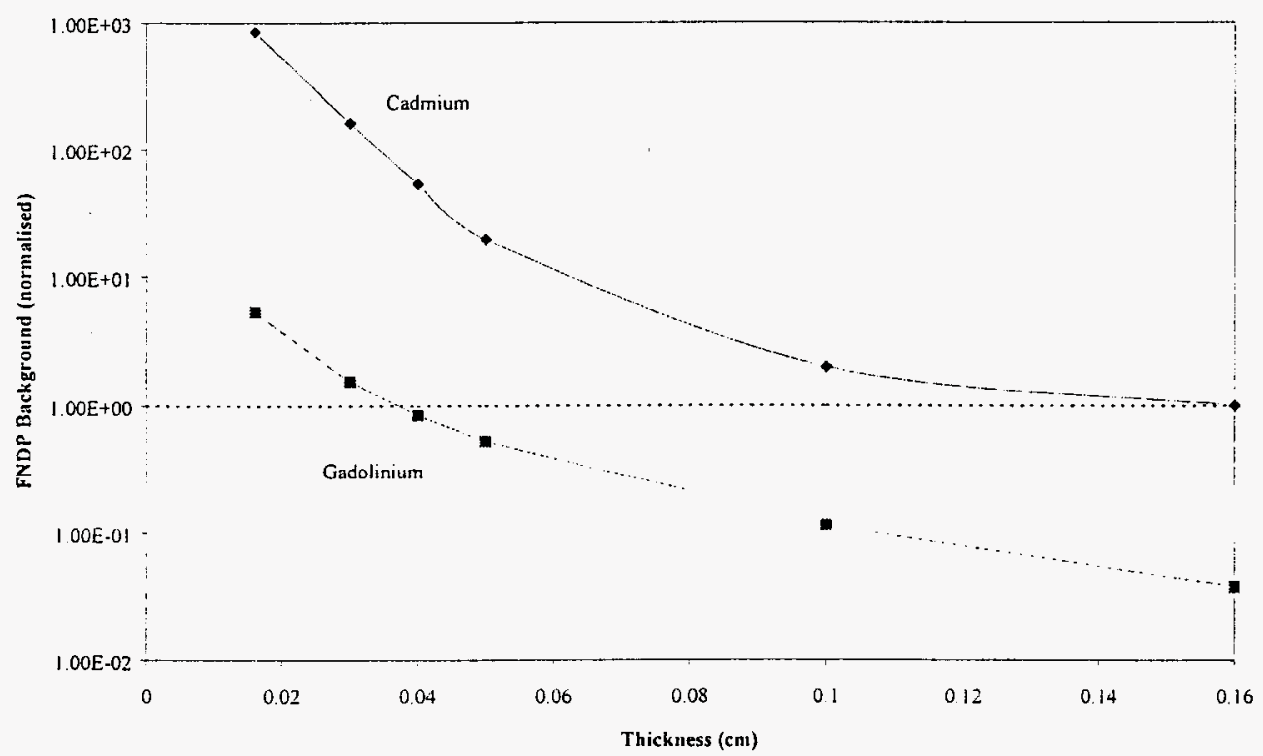

Figure 10. Estimated FNDP background count rate as a function of thickness of Cd or $\mathrm{Gd}_{2} \mathrm{O}_{2} \mathrm{~S}$, normalized to the default case of $1.6 \mathrm{~mm}$ thick cadmium. 


\begin{tabular}{|l|l|r|}
\hline & Canberra Industries, ACK\# D0043 & 33296 B \\
\hline Created: 29-September-2000 & IBIS Project & Revision B \\
\hline Revised: 13-January-2003 & Preliminary Design Study & Page 68 of 156 \\
\hline
\end{tabular}

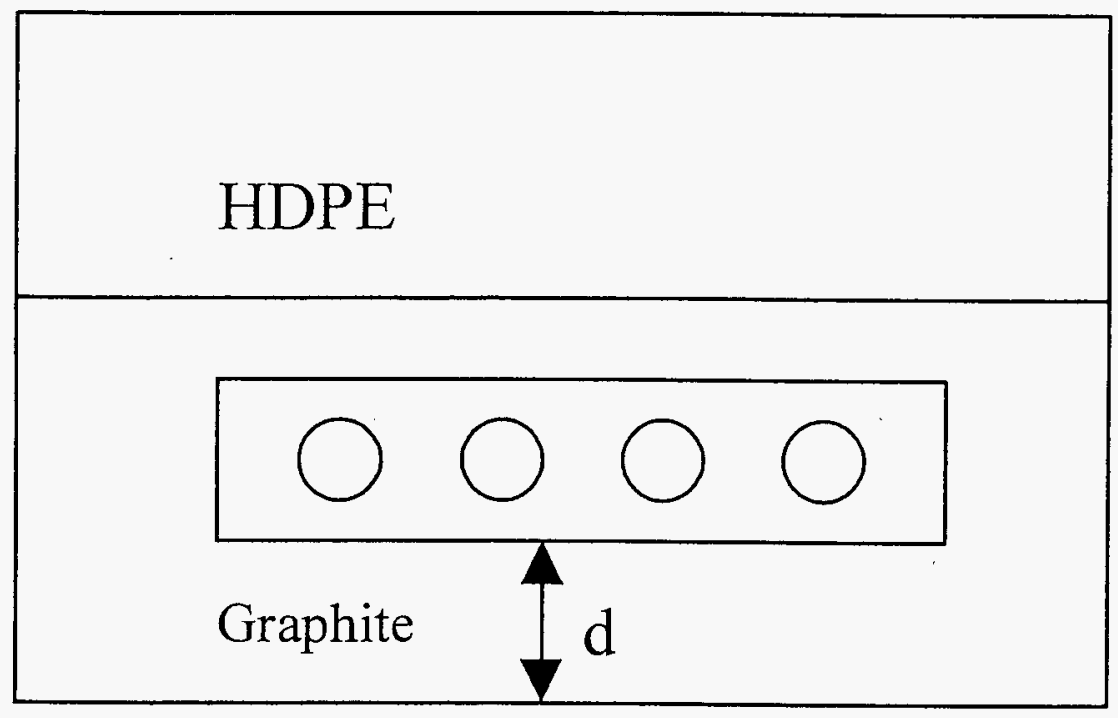

Figure 11. Position of FNDP's relative to DDA chamber walls.

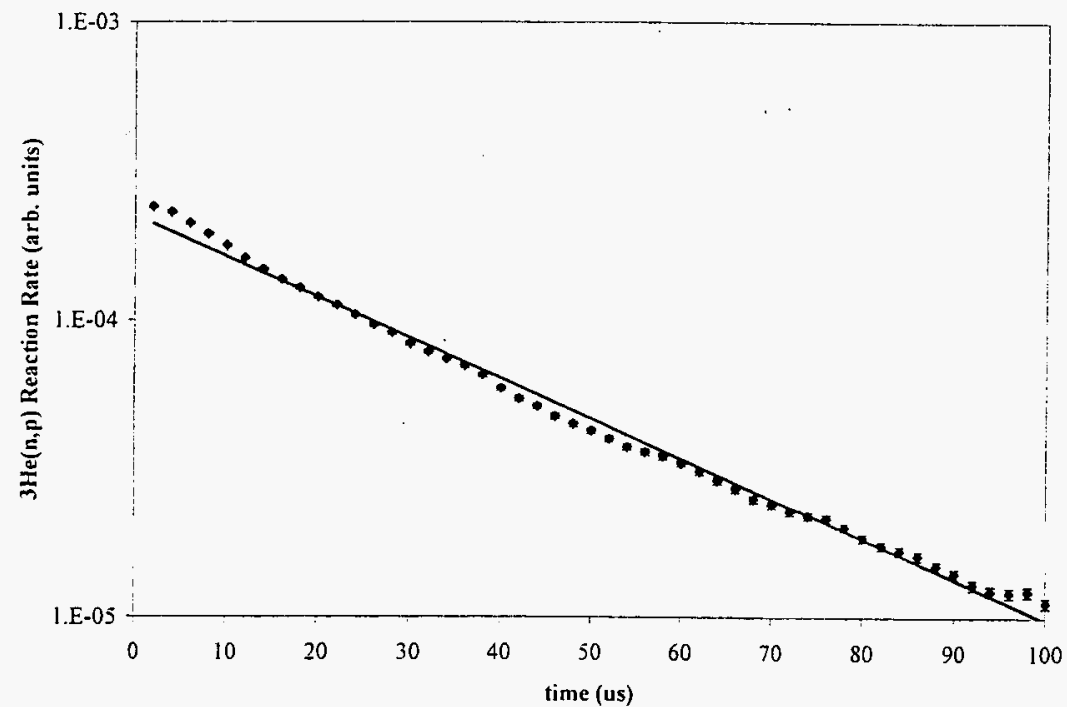

Figure 12. MCNPTM calculated die-away profile $(0-100 \mu \mathrm{s})$ for FNDP's, for the empty box model, with an FNDP embedding depth of $14 \mathrm{~mm}$. Die-away time fit $\approx 32 \mu \mathrm{s}$. 


\begin{tabular}{|l|l|r|}
\hline & Canberra Industries, ACK\# D0043 & 33296 B \\
\hline Created: 29-September-2000 & IBIS Project & Revision B \\
\hline Revised: 13-January-2003 & Preliminary Design Study & Page 69 of 156 \\
\hline
\end{tabular}

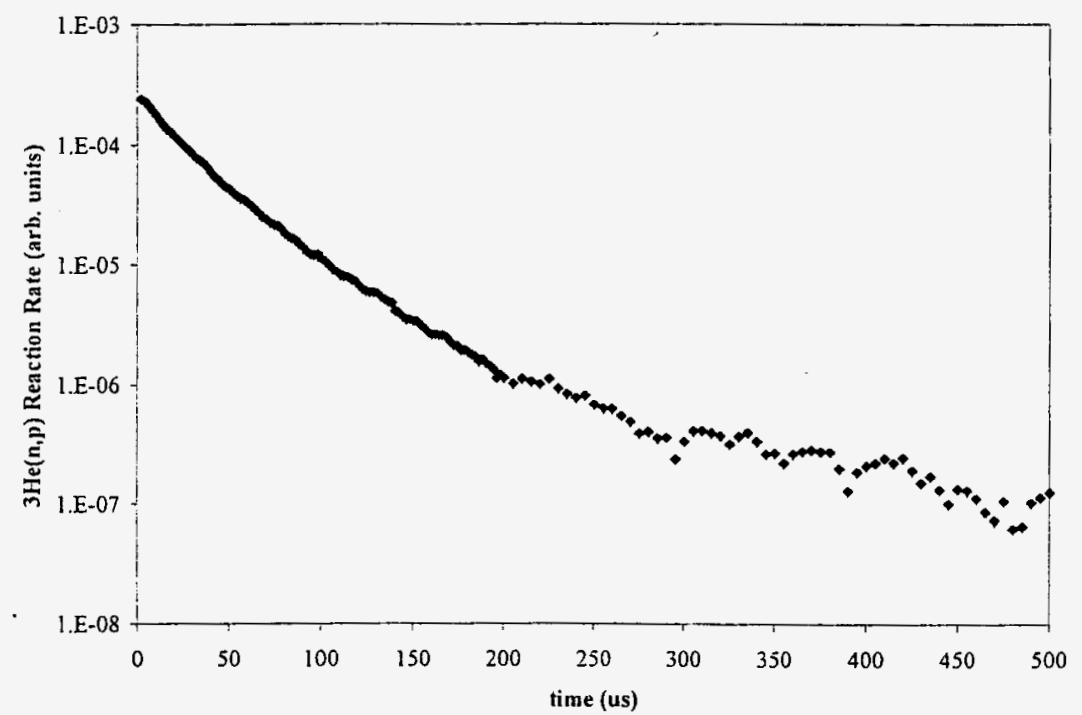

Figure 13. MCNPTM calculated die-away profile $(0-500 \mu \mathrm{s})$ for FNDP's, for the empty box model, with an FNDP embedding depth of $14 \mathrm{~mm}$.

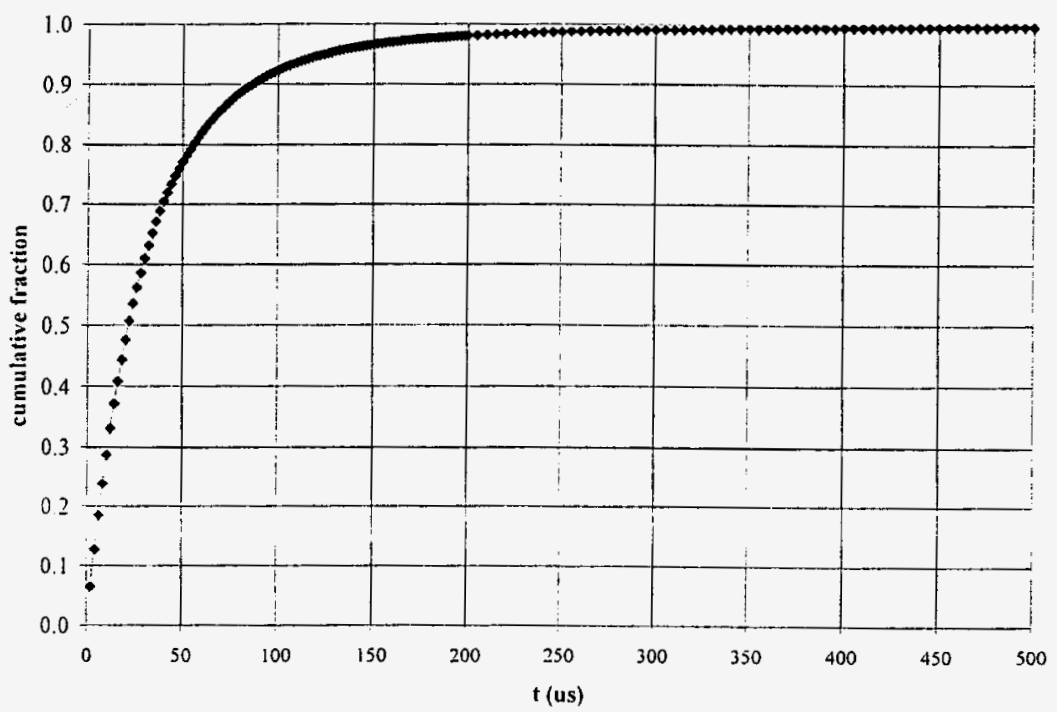

Figure 14. Cumulative fraction of the total integrated response for the FNDP's, based on the time profile shown in Figure 13. 


\begin{tabular}{|l|l|r|}
\hline & Canberra Industries, ACK\# D0043 & 33296 B \\
\hline Created: 29-September-2000 & IBIS Project & Revision B \\
\hline Revised: 13-January-2003 & Preliminary Design Study & Page 70 of 156 \\
\hline
\end{tabular}
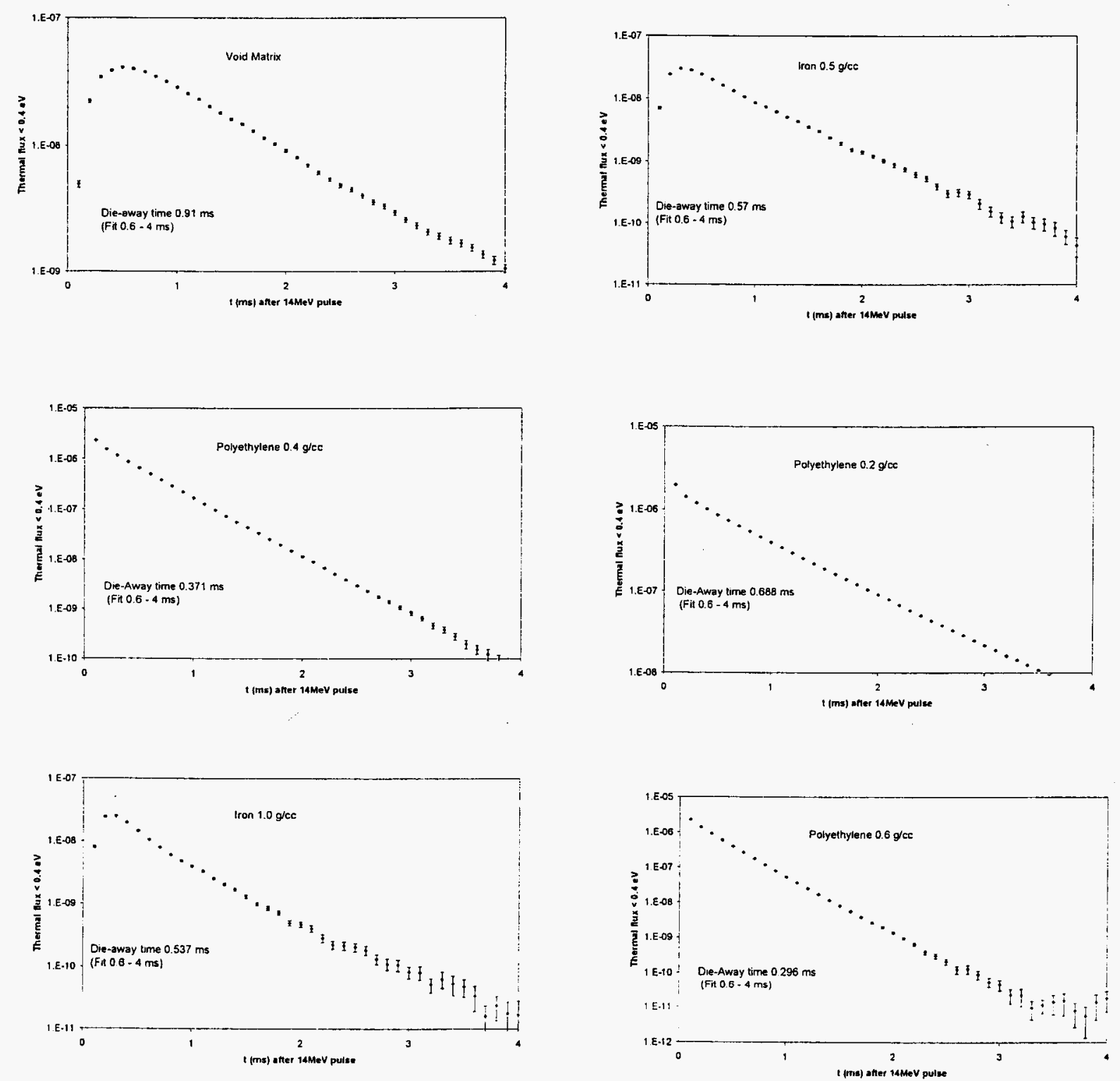

Figure 15. Time profiles for the thermal $(<0.4 \mathrm{eV})$ interrogating flux, averaged over the box volume. The irradiation position is centered on the back face, the FNDP embedding depth is 14 $\mathrm{mm}$ and the graphite thickness is $150 \mathrm{~mm}$. 


\begin{tabular}{|l|l|r|}
\hline & Canberra Industries, ACK\# D0043 & 33296 B \\
\hline Created: 29-September-2000 & IBIS Project & Revision B \\
\hline Revised: 13-January-2003 & Preliminary Design Study & Page 71 of 156 \\
\hline
\end{tabular}

\subsection{DDA response results}

Count rate and LLD:

Because we have access to DDA experimental data against which we are able to benchmark the $\mathrm{MCNP}^{\mathrm{TM}}$ modeling, absolute calculations are not required; only relative quantities are needed. This also means that basic nuclear data (such as ${ }^{235} \mathrm{U}$ microscopic cross-section and prompt fission neutron yields) is not required.

The results for the base model geometry are summarized in Tables $2-6$. This shows the matrix variability of response and also the Lower Limits of Detection. The $3 \sigma$ LLD results have been scaled from experimental data with a prototype $200 l$ drum DDA chamber at Harwell, against which MCNPTM has been benchmarked [Chard, 1992]. The DDA mode LLD is inversely proportional to the specific response (counts per second per unit mass of ${ }^{235} \mathrm{U}$ ), and to the square root of the FNDP background count rate (b). For the present purposes, $b$ is assumed to be proportional to $\varepsilon$. The specific DDA response is assumed to be proportional to the flux.efficiency $(\varphi . \varepsilon)$ product. Defining the subscripts "HIL" and "IBIS" to denote respectively the Harwell prototype $200 /$ DDA chamber, and the IBIS chamber model, the specific DDA response, c, (c/s/g ${ }^{235} \mathrm{U}$ ) is given by Equation 16 . Here, $c_{H I L}$ is the measured specific count rate for the Harwell chamber.

DDA measurement time intervals of $0.8-10$ ms have been selected for the DDA count rate predictions from now onwards. The start time of $0.8 \mathrm{~ms}$ is somewhat conservative, allowing plenty of time for the nucleonics to recover from the $14 \mathrm{MeV}$ burst. The quoted results from now onwards refer to this specified DDA counting interval.

$$
c_{I B I S}=c_{H I L}\left(\frac{\phi_{I B I S}}{\phi_{H I L}} \cdot \frac{\varepsilon_{I B I S}}{\varepsilon_{H I L}}\right)
$$

Eq 16.

The LLD is proportional to $1 / \mathrm{c}$ and also to the square root of the background count rate. The background count rate, $\mathrm{B}$, is assumed to be proportional to the detection efficiency. Thus the LLD can be expressed by Equation 17.

$$
L L D_{I B I S}=L L D_{H I L}\left(\frac{\phi_{H I L}}{\phi_{I B I S}} \cdot \frac{\varepsilon_{H I L}}{\varepsilon_{I B I S}}\right) \cdot\left(\frac{\varepsilon_{I B I S}}{\varepsilon_{H I L}}\right)^{0.5}
$$

The die-away profile for the DDA thermal $(<0.4 \mathrm{eV})$ interrogating flux is illustrated in Figure 15. These profiles are taken from the MCNPTM results for the back wall irradiation position, with $150 \mathrm{~mm}$ graphite and an FNDP embedding depth of $14 \mathrm{~mm}$. The thermal flux builds up in the first few tenths of a millisecond, and then follows an exponential decay. Note that the build up time $\left(\tau_{b}\right)$ is reduced by the matrix, and in particular for a moderating matrix, this reduces to $<$ $0.1 \mathrm{~ms}$. The die-away time $\left(\tau_{\mathrm{d}}\right)$ is influenced most dramatically by the thermal neutron absorption 


\begin{tabular}{|l|l|r|}
\hline & Canberra Industries, ACK\# D0043 & 33296 B \\
\hline Created: 29-September-2000 & IBIS Project & Revision B \\
\hline Revised: 13-January-2003 & Preliminary Design Study & Page 72 of 156 \\
\hline
\end{tabular}

properties of the matrix, and is reduced considerably by the iron matrices. The die-away time $\tau_{\mathrm{d}}$, is proportional to the reciprocal of the macroscopic thermal neutron absorption cross-section of the matrix. These flux profile characteristics are summarized in Table 5.

The predicted absolute count rates are summarized in Table 6. The interrogating flux $\varphi$ (calculated over the measurement time interval of interest) was folded with the microscopic total fission cross-section for ${ }^{235} \mathrm{U}$, and multiplied by the thermal neutron induced prompt fission neutron $v$ value (taken to be 2.414)[Chard and Croft, 1997]. The basic parameter calculated, is the number of counts observed (integrated over the specified DDA counting interval), per gram of ${ }^{235} \mathrm{U}$, per source neutron.

The results in Table 6 assume a $14 \mathrm{MeV}$ neutron source strength of $1 \times 10^{8} \mathrm{n} \cdot \mathrm{s}^{-1}$, and represent the observed count rate per second of real time, for this source strength. To determine the observed counts per gram ${ }^{235} \mathrm{U}$ per $14 \mathrm{MeV}$ pulse, the numbers in Table 6 should be divided by $\left(\mathrm{N}_{0} . \mathrm{f}\right)$ where $\mathrm{N}_{0}$ is the number of neutrons per pulse, and $\mathrm{f}$ is the pulse frequency. It is expected that an operating regime will be adopted for the IBIS system where $f=50 \mathrm{~Hz}$, with a $14 \mathrm{MeV}$ neutron source strength of $1 \times 10^{8} \mathrm{n} \cdot \mathrm{s}^{-1}$, (that is, $\mathrm{N}_{0}=2 \times 10^{6}$ ).

We can calculate the count rate for ${ }^{239} \mathrm{Pu}$, from the ${ }^{235} \mathrm{U}$ values presented in Table 6, by taking into account the different fission cross-sections and prompt neutron yields for the two isotopes. Using the nuclear data presented in [Chard and Croft, 1997], based on the thermal Maxwellian fission cross-sections and prompt neutron yield data for thermal neutron induced fission, the ${ }^{239} \mathrm{Pu} /{ }^{235} \mathrm{U}$ ratio is 1.6105 ( \pm 0.0069 ). The expected counts per unit mass of ${ }^{239} \mathrm{Pu}$ can therefore be obtained by multiplying the numbers in Table 6 by this constant.

Table 2. Detection efficiency $(\varepsilon)$ results for different configurations studied (uniform ${ }^{240} \mathrm{Pu}$ spontaneous fission source).

\begin{tabular}{|c|c|c|c|}
\hline & \multicolumn{3}{|c|}{ FNDP Embedding Depth (mm) } \\
Matrix & 14 & 44 & 74 \\
\hline Void & 22.07 & 20.6 & 18.5 \\
Iron $\left(0.5{\left.\mathrm{~g} . \mathrm{cm}^{-3}\right)}_{\text {Iron }\left(1.0{\left.\mathrm{~g} . \mathrm{cm}^{-3}\right)}^{-3}\right.}^{23.15}\right.$ & 23.84 & 21.56 & 19.43 \\
Poly $\left(0.2{\left.\mathrm{~g} . \mathrm{cm}^{-3}\right)}^{-3}\right.$ & 6.93 & 22.14 & 19.87 \\
Poly $\left(0.4 \mathrm{~g} . \mathrm{cm}^{-3}\right)$ & 3.64 & 5.4 & 4.4 \\
\hline
\end{tabular}




\begin{tabular}{|l|l|r|}
\hline & Canberra Industries, ACK\# D0043 & 33296 B \\
\hline Created: 29-September-2000 & IBIS Project & Revision B \\
\hline Revised: 13-January-2003 & Preliminary Design Study & Page 73 of 156 \\
\hline
\end{tabular}

Table 3. Flux $\times$ efficiency $(\varphi . \varepsilon)$ results $\left(\right.$ n. $\left.\mathrm{cm}^{-2}\right)$ for different configurations studied (uniform ${ }^{240} \mathrm{Pu}$ spontaneous fission source). The values normalized to the void matrix, are shown in brackets. The flux is integrated from $0.8-10 \mathrm{~ms}$, and $<0.4 \mathrm{eV}$.

\begin{tabular}{|c|c|c|c|}
\hline & \multicolumn{3}{|c|}{ FNDP Embedding Depth (mm) } \\
Matrix & 14 & 44 & 74 \\
\hline Void & $7.26 \mathrm{E}-08(1.0)$ & $1.41 \mathrm{E}-07(1.0)$ & $2.34 \mathrm{E}-07(1.0)$ \\
Iron $\left(0.5 \mathrm{~g} . \mathrm{cm}^{-3}\right)$ & $1.83 \mathrm{E}-08(0.25)$ & $2.75 \mathrm{E}-08(0.19)$ & $3.88 \mathrm{E}-08(0.17)$ \\
Iron $\left(1.0 \mathrm{~g} . \mathrm{cm}^{-3}\right)$ & $8.77 \mathrm{E}-09(0.12)$ & $1.26 \mathrm{E}-08(0.09)$ & $1.71 \mathrm{E}-08(0.07)$ \\
Poly $\left(0.2 \mathrm{~g} . \mathrm{cm}^{-3}\right)$ & $2.20 \mathrm{E}-07(3.0)$ & $1.97 \mathrm{E}-07(1.39)$ & $1.83 \mathrm{E}-07(0.78)$ \\
Poly $\left(0.4 \mathrm{~g} . \mathrm{cm}^{-3}\right)$ & $3.35 \mathrm{E}-08(0.46)$ & $3.08 \mathrm{E}-08(0.22)$ & $2.82 \mathrm{E}-08(0.12)$ \\
\hline
\end{tabular}

Table 4. $3 \sigma \mathrm{LLD}$ results ( $\mathrm{g}^{235} \mathrm{U}$ ) for different configurations studied (uniform distribution within the box). These results are for a $1 \times 10^{8} \mathrm{n} . \mathrm{s}^{-1} 14 \mathrm{MeV}$ neutron source, with a 200 second assay time, and apply for a clean drum, with no Pu content (which would elevate the observed background count rate).

\begin{tabular}{|c|c|c|c|}
\hline & \multicolumn{3}{|c|}{ FNDP Embedding Depth (mm) } \\
Matrix & 14 & 44 & 74 \\
\hline Void & $4.59 \mathrm{E}-02$ & $2.28 \mathrm{E}-02$ & $1.30 \mathrm{E}-02$ \\
Iron $\left(0.5{\left.\mathrm{~g} . \mathrm{cm}^{-3}\right)}^{-3}\right.$ & $1.86 \mathrm{E}-01$ & $1.20 \mathrm{E}-01$ & $8.05 \mathrm{E}-02$ \\
Iron $\left(1.0{\left.\mathrm{~g} . \mathrm{cm}^{-3}\right)}^{-3}\right.$ & $3.94 \mathrm{E}-01$ & $2.65 \mathrm{E}-01$ & $1.84 \mathrm{E}-01$ \\
Poly $\left(0.2{\left.\mathrm{~g} . \mathrm{cm}^{-3}\right)}^{-3}\right.$ & $8.48 \mathrm{E}-03$ & $8.37 \mathrm{E}-03$ & $8.13 \mathrm{E}-03$ \\
Poly $\left(0.4 \mathrm{~g} . \mathrm{cm}^{-3}\right)$ & $4.04 \mathrm{E}-02$ & $3.93 \mathrm{E}-02$ & $3.83 \mathrm{E}-02$ \\
\hline
\end{tabular}

Table 5. Summary of DDA thermal neutron interrogating flux time profile characteristics. These results are for the back face irradiation position, with $150 \mathrm{~mm}$ thick graphite and an FNDP embedding depth of $14 \mathrm{~mm}$.

\begin{tabular}{|c|c|c|}
\hline Box Matrix & Build up time $\tau_{\mathrm{b}}(\mathrm{ms})$ & Die-away time $\tau_{\mathrm{d}}(\mathrm{ms})$ \\
\hline Void & 0.6 & 0.91 \\
Iron $0.5 \mathrm{~g} \cdot \mathrm{cm}^{-3}$ & 0.3 & 0.57 \\
Iron $1.0 \mathrm{~g} . \mathrm{cm}^{-3}$ & 0.3 & 0.54 \\
Polyethylene $0.2 \mathrm{~g} . \mathrm{cm}^{-3}$ & $<0.1$ & 0.69 \\
\hline
\end{tabular}




\begin{tabular}{|l|l|r|}
\hline & Canberra Industries, ACK\# D0043 & 33296 B \\
\hline Created: 29-September-2000 & IBIS Project & Revision B \\
\hline Revised: 13-January-2003 & Preliminary Design Study & Page 74 of 156 \\
\hline
\end{tabular}

Table 6. Calculated DDA count rates $\left(\mathrm{c} / \mathrm{s} / \mathrm{g}^{235} \mathrm{U}\right.$ ) for different configurations studied (uniform distribution within the box). The values normalized to the void matrix, are shown in brackets. These results are for a $1 \times 10^{8} \mathrm{n} \cdot \mathrm{s}^{-1} 14 \mathrm{MeV}$ neutron source. The count rates are per second of real time.

\begin{tabular}{|c|c|c|c|}
\hline & \multicolumn{3}{|c|}{ FNDP Embedding Depth (mm) } \\
Matrix & 14 & 44 & 74 \\
\hline Void & $22.6(1.0)$ & $44.0(1.0)$ & $72.8(1.0)$ \\
Iron $\left(0.5{\left.\mathrm{~g} . \mathrm{cm}^{-3}\right)}^{-3}\right.$ & $5.70(0.25)$ & $8.55(0.19)$ & $12.1(0.17)$ \\
Iron $\left(1.0{\left.\mathrm{~g} . \mathrm{cm}^{-3}\right)}_{\text {Poly }\left(0.2 \mathrm{~g} . \mathrm{cm}^{-3}\right)}^{2.73(0.12)}\right.$ & $3.91(0.09)$ & $5.34(0.07)$ \\
Poly $\left(0.4 \mathrm{~g} . \mathrm{cm}^{-3}\right)$ & $68.5(3.0$ & $61.3(1.39)$ & $56.9(0.78)$ \\
\hline
\end{tabular}

\section{Spatial variability of response:}

We note that the spatial variation exhibited by the detection efficiency is expected to be much less than that for the interrogating flux, and has therefore been ignored for the present purposes. The detection efficiency results presented above are therefore based on a uniform distribution of fission neutrons throughout the container. By way of illustration, the spatial variability of the interrogating thermal neutron flux has also been analyzed. As we have seen, the box volume is divided, for the purposes of the MCNP ${ }^{\mathrm{TM}}$ modeling, into 45 equal volume rectangular voxels, in an array of $3 \times 3 \times 5(\mathrm{~h} \times \mathrm{w} \times \mathrm{l})$. We can visualize the degree of penetration of the flux into the matrix by analyzing the relative flux in the 3 voxels adjacent to the front face irradiation position. This irradiation position is at the center of the front face, and centered about the near voxel. A Near - to - Far ratio can be formed using the Near (Adjacent) voxel and the Far (on the opposite side - the back - of the crate). For simplicity and speed, this analysis has been performed using the gross (integrated over all times) thermal flux $(<0.4 \mathrm{eV})$. The results are given in Tables 7 and 8.

Table 7. Middle / Near voxel gross thermal flux ratios for central irradiation at the back face. The 1 sigma statistical uncertainties (from MCNPTM )on the last significant figure are given in brackets.

\begin{tabular}{|c|c|c|c|}
\hline & \multicolumn{3}{|c|}{ FNDP Embedding Depth (mm) } \\
Matrix & 14 & 44 & 74 \\
\hline Void & $1.07(6)$ & $0.97(4)$ & $0.95(3)$ \\
Fe 0.5 & $0.47(5)$ & $0.43(3)$ & $0.44(3)$ \\
Fe 1.0 & $0.31(4)$ & $0.21(3)$ & $0.15(2)$ \\
Poly 0.2 & $0.346(5)$ & $0.353(6)$ & $0.448(6)$ \\
Poly 0.4 & $0.132(2)$ & $0.132(2)$ & $0.128(2)$ \\
\hline
\end{tabular}




\begin{tabular}{|l|l|r|}
\hline & Canberra Industries, ACK\# D0043 & 33296 B \\
\hline Created: 29-September-2000 & IBIS Project & Revision B \\
\hline Revised: 13-January-2003 & Preliminary Design Study & Page 75 of 156 \\
\hline
\end{tabular}

Table 8. Far / Near voxel gross thermal flux ratios for central irradiation at the back face. The 1 sigma statistical uncertainties (from MCNPтM TM)on the last significant figure are given in brackets.

\begin{tabular}{|c|c|c|c|}
\hline & \multicolumn{3}{|c|}{ FNDP Embedding Depth (mm) } \\
\hline Matrix & 14 & 44 & 74 \\
\hline Void & $1.09(6)$ & $0.92(4)$ & $0.93(3)$ \\
Fe 0.5 & $0.39(5)$ & $0.43(4)$ & $0.54(4)$ \\
Fe 1.0 & $0.21(3)$ & $0.21(2)$ & $0.22(2)$ \\
Poly 0.2 & $0.089(2)$ & $0.095(2)$ & $0.095(2)$ \\
Poly 0.4 & $0.0140(6)$ & $0.0144(7)$ & $0.0137(6)$ \\
\hline
\end{tabular}

The results in Tables 7 and 8 show that the flux is spatially rather uniform in the case of a void matrix ( $9 \%$ maximum variation). This should be an encouraging result for light waste matrices. For the iron and polyethylene matrices, the response is considerably depressed at the middle and far voxels, relative to the near voxel. By rotating the crate through 180 degrees and averaging over the two results, the depression at the remote voxels can be compensated, leaving only the depression in the central regions. The central voxel flux depression for the $0.5 \mathrm{~g} . \mathrm{cm}^{-3}$ matrix is of the order of a factor of 2.5 . For the $1.0 \mathrm{~g} \mathrm{.cm}^{-3}$ matrix, this factor is approximately 5 . The effect of the FNDP embedding depth on the flux depression appears to be second order.

\subsubsection{Optimization of FNDP embedding depths}

The optimum FNDP embedding depth (d) within the graphite walls, is a compromise between the requirements for a high detection efficiency, $\varepsilon$ (for the Passive mode PNCC assay, where the specific response is proportional to $\varepsilon^{2}$ ), and a high Flux $x$ efficiency Product, $\varphi . \varepsilon$, (the DDA specific response is proportional to $\varphi . \varepsilon$ ). A small embedding depth means that the detectors are close to the sample, leading to a high detection efficiency. However this conflicts with the requirements for a high thermal interrogating flux, as the smaller the embedding depth, the greater is the poisoning by the cadmium. As the cadmium changes the energy spectrum of the interrogating flux, the embedding depth also affects the matrix variability of the DDA response. For example, a small amount of poisoning leads to a highly thermal flux, so that a moderating matrix cannot increase the thermal flux component, and hence the ${ }^{235} \mathrm{U}$ specific response, by a large amount. Moreover, a highly poisoned flux means that the epithermal / fast component of the interrogating flux is relatively high, such that a slightly moderating matrix can have a large effect on the response.

In practice it is considered that the flux and matrix effects are the most important factors which will contribute to the selection of the embedding depth. These will have a direct impact on the active mode performance, in terms of the measurement accuracy, as they relate to the matrix compensation uncertainty and fissile material position variations. The optimization for the Passive mode assay is far simpler, and one has only to ensure that an adequate detection efficiency is maintained, to achieve the required performance. A total detection efficiency 


\begin{tabular}{|l|l|r|}
\hline & Canberra Industries, ACK\# D0043 & 33296 B \\
\hline Created: 29-September-2000 & IBIS Project & Revision B \\
\hline Revised: 13-January-2003 & Preliminary Design Study & Page 76 of 156 \\
\hline
\end{tabular}

requirement of at least $14 \%$ has been specified in the RFT. As the detection efficiency is determined by the moderation within the FNDP moderator material itself, the graphite has only a second order effect; consequently the FNDP embedding depth has a negligible effect on the matrix effects and hence the measurement uncertainty, for the passive mode assay.

\subsection{Practical DDA counting intervals}

It is expected that a pulse frequency of $50 \mathrm{~Hz}$ will be used for the IBIS system. This leaves $20 \mathrm{~ms}$ available for counting, between pulses. With a predicted die-away time of no more than $\approx 1 \mathrm{~ms}$ (see Figure 15), the DDA counting gate needs only extend out to a few ms, to capture $>99 \%$ of the total DDA signal. For this reason it is expected that the DDA gate interval needs to be no more than $5 \mathrm{~ms}$ in length. There will be no benefit in having a longer interval, as the increase in signal will be very small $(<1 \%)$. The background count would increase, thereby unnecessarily elevating the LLD.

\subsubsection{Delayed Neutron Counting}

Implementing a DDA counting interval of say $0.8-5.8$, with a $14 \mathrm{MeV}$ pulse frequency of 50 $\mathrm{Hz}$, this leaves $1 / 50-0.0058=0.0142$ seconds, or $14.2 \mathrm{~ms}$ of time remaining between pulses. This opens up the option of counting delayed fission neutrons in the time between $14 \mathrm{MeV}$ pulses, after allowing sufficient time for the prompt fission neutrons to die-away to a negligible level compared to the inter - pulse ambient neutron background signal. The delayed neutron count mode requires that the count gates be set appropriately but otherwise no additional hardware is required.

Conventional active neutron interrogation in the DDA mode is a sensitive technique for assaying fissile material, as it utilizes the high fission cross-section associated with the fully thermalized interrogating flux over the DDA counting interval (say $0.8-5.8 \mathrm{~ms}$ for the present purposes). However if fissile material is present in lump or aggregate form, then there is the possibility of severe underestimation due to the effects of sample self -shielding [Chard and Croft, 1995, pp 189-194].

A dual active neutron interrogation (DANI) variant of standard DDA counting for 55 gallon waste drums was developed at Harwell [Armitage and Cogbill, 1989] to investigate the possibility of correcting for the self shielding, based on delayed neutron counting. By counting delayed neutrons, which are emitted on a timescale of typically many seconds after the initial fission event, one is effectively sampling from an interrogating flux which includes the unthermalized component at short times $(0-0.8 \mathrm{~ms})$ after the $14 \mathrm{MeV}$ pulse. The delayed neutron (DN) response is therefore due to a thermal + epithermal flux and is therefore subject to less severe self shielding than DDA counting. The DN/DDA ratio is therefore diagnostic of the amount of self shielding in the sample.

While delayed neutron counting may be useful under certain restricted circumstances to provide a warning of the presence of large lumps of fissile material, it is generally not viable as a 


\begin{tabular}{|l|l|r|}
\hline & Canberra Industries, ACK\# D0043 & 33296 B \\
\hline Created: 29-September-2000 & IBIS Project & Revision B \\
\hline Revised: 13-January-2003 & Preliminary Design Study & Page 77 of 156 \\
\hline
\end{tabular}

quantitative technique for self shielding compensation. The method relies on the assumption of uniform, well characterized matrix, and also a single fissile material lump. In addition, moderating matrices rapidly rethermalize the flux, leading to a deterioration in the sensitivity of the DN/DDA ratio to self-shielding. In practice this means that only a modest quantity of moderating material will render the DANI method ineffective. The DN/DDA sensitivity to self shielding is low for moderate self shielding, and only improves as the self shielding becomes more severe. It is for this reason that the technique could only be used as a flag to warn of the likely presence of severely self shielded material.

However there is very little overhead associated with counting delayed neutrons, just requiring an additional, extended time window so it is considered worthwhile logging the delayed neutron response for later analysis. A proposed $\mathrm{DN}$ counting interval would be $10-19 \mathrm{~ms}$ after the pulse. A delay of $10 \mathrm{~ms}$ is used instead of $5 \mathrm{~ms}$, to make absolutely sure that there is no residual DDA signal. After $20 \mathrm{~ms}$, the next pulse arrives.

The DN/DDA response ratio is given by Equation 18.

$$
\frac{D N}{D D A}=\frac{\varepsilon_{d}}{\varepsilon_{p}} \cdot f \cdot \frac{Y}{\frac{v_{p}}{v_{p}}} \cdot \frac{\int_{0}^{\infty} \phi(E, t) \cdot \sigma_{f}(E, t) \cdot d E \cdot d t}{\int_{0.7}^{5 m s} \phi(E, t) \cdot \sigma_{f}(E, t) \cdot d E \cdot d t}
$$

where $\varepsilon_{d}$ and $\varepsilon_{p}$ are the detection efficiencies for delayed and prompt fission neutrons respectively, $\mathrm{f}$ is the observable fraction of delayed neutrons, $\mathrm{Y}$ is the mean delayed neutron yield per fission, $\bar{v}_{p}$ is the mean prompt neutron yield per fission. The $\phi . \sigma_{f}$ integrals are the fission rates, calculated by MCNPTM. The observable fraction $f$ can be calculated by modeling the delayed neutron precursor equilibrium [Chard, 1991]. To first order, the value of $\mathrm{f}$ can be taken to be equal to the ratio of the DN counting interval to the inter - pulse time, that is $(0.019 \mathrm{~ms}$ $0.010 \mathrm{~ms}$ ) $/ 0.020 \mathrm{~ms}=0.45$. Taking data for thermal neutron induced fission in [Chard and Croft, 1997], the $Y / \overline{v_{p}}$ ratio is equal to $0.0162 / 2.414=0.00671$. If we neglect the difference in detection efficiencies between delayed and fission neutrons (experience shows this to be valid to first order), then the first three factors in Equation 18 reduce to a factor of 0.003 .

By way of illustration, the fission rate ratio (the ratio of integrals in Equation 18) has been calculated by MCNPrM , for the case of a $150 \mathrm{~mm}$ graphite layer, with an FNDP embedding depth of $14 \mathrm{~mm}$, with a void matrix, and the back face irradiation position. The ratio is 2.25 $( \pm 0.05)$, leading to a DN/DDA ratio of 0.00675 . The delayed neutron count rate is therefore obtained by multiplying the value in Table $6(22.6)$ by this factor. This serves to illustrate the magnitude of the expected DN count rates. The DN/DDA ratio is matrix dependent, owing to the matrix - dependent epithermal flux at short times relative to the $14 \mathrm{MeV}$ pulse. 


\begin{tabular}{|l|l|r|}
\hline & Canberra Industries, ACK\# D0043 & 33296 B \\
\hline Created: 29-September-2000 & IBIS Project & Revision B \\
\hline Revised: 13-January-2003 & Preliminary Design Study & Page 78 of 156 \\
\hline
\end{tabular}

\subsection{Calibration Methodology}

Extensive calibrations will be performed using surrogate homogeneous matrices representative of realistic waste streams, with fissile samples placed at a range of positions inside each container. In the general case in which matrix compensation techniques are employed, it is expected that of the order of $10-20$ different matrices will be required to adequately cover the expected range of properties for the real wastes. However the calibrations will be performed for only a single, selected box type; since there is a large number of box sizes which are required to be assayed in the IBIS system, measurements at a range of positions in all 10-20 matrices, for each box type, will not be feasible on time / costs grounds. Therefore we expect that mathematical modeling techniques will be used to calculate the responses for the complete set of sample position / matrix type / box type combinations, benchmarked against the calibration measurements actually performed.

It is expected that the system will be calibrated using a range of representative $\mathrm{Pu}$ and $\mathrm{U}$ standards for the passive and active mode assays respectively. By making measurements across the full range of expected masses of ${ }^{240} \mathrm{Pu}_{\text {equiv }}$ and ${ }^{235} \mathrm{U}$, the response linearity can be demonstrated at a fixed position within a given matrix. This functional form will then be superimposed on the measured single mass calibrations at each position in each matrix, assuming that the response linearity is characteristic of the range of fissile samples considered, and is not affected by position / matrix variations.

Often factory acceptance tests are conducted using depleted uranium as a surrogate for ${ }^{239} \mathrm{Pu}$ and ${ }^{252} \mathrm{Cf}$ as a surrogate for ${ }^{240} \mathrm{Pu}_{\text {eff. }}$ Calculated equivalence values are used to convert the measured quantities into the desired variables. Final calibration and verification of the system is preferably performed using actual special nuclear material samples as similar to those likely to be encountered in practice. To reduce the need to transport SNM between different DOE sites these measurements are usually performed as a type test at LANL or at the destination site.

The calibration will be made directly on SWBs and / or B25s depending on availability and will cover dry combustibles, wet combustibles, plastics, and light metals as a minimal set. The calibration will be extended, by either direct measurement or calculation.

It is expected that extensive use will be made of mathematical modeling (for example the Monte Carlo modeling code MCNPTM TM) and existing data derived from NDA field experience, to bound the potential response perturbations caused by sample self-multiplication and self shielding, for the passive and active mode assays respectively. For example, if the most extreme (largest) fissile material lump with the greatest ${ }^{235} \mathrm{U}$ enrichment is known, then the most severe self - shielding factor can be calculated using proven techniques, as a bounding value. The other extreme would be the case of infinitely diluted fissile material with no self -shielding. By assigning a fixed percentage confidence interval to these bounding cases, the contribution to the overall Total Measurement Uncertainty (TMU) can be determined. The same arguments may be applied for self multiplication in the case of passive neutron assays. 


\begin{tabular}{|l|l|r|}
\hline & Canberra Industries, ACK\# D0043 & 33296 B \\
\hline Created: 29-September-2000 & IBIS Project & Revision B \\
\hline Revised: 13-January-2003 & Preliminary Design Study & Page 79 of 156 \\
\hline
\end{tabular}

\subsubsection{Known matrix calibration}

If the waste originates from a stream where good sorting, treatment, segregation and record keeping practices have been established, an item presented for assay may have associated with it good a priori information regarding its general contents. In such cases it may be appropriate to select a calibration pertinent to that particular category or classification of waste from a database of calibrations known to the system. These can have been generated by measurements on realistic simulated waste forms representative of the kind, or else generated numerically by a mathematical model. The means of selecting a predetermined calibration coefficient from the data base could be based on the Item Description Code (IDC) and the Matrix Parameter Characteristics (MPC) information. When contents of the boxes are unknown or undeclared the system must determine what calibration coefficients to use.

\subsubsection{Wide-range calibration}

In this approach the assay system derives matrix compensation factors from the data it measures, appropriate to the particular item being measured. Relationships between the measured quantities and the correction factors are determined through empirical models fitted to experimental calibration data. The calibration set will be designed to span the full range of density, moderation, absorption, inhomogeneity properties expected to be encountered in practice. The system will be self-policing to the extent that when the matrix characterization parameters fall outside the range in which the system was calibrated, it will not attempt to extrapolate beyond the boundary but instead will output a message to the effect that the container is unsuitable for reliable quantitative measurement by these techniques and calls for a review by a (human) expert.

To perform a wide range calibration for the case of homogeneous drums of different matrix type sufficient to allow volume weighted average or VWA (that is global) matrix compensation factors to be derived for uniformly dispersed fissile material, a minimum of 10 to 20 boxes will be needed. For $200 l$ drum calibrations measurements are typically taken with the source located at 9 or more positions within the drum depending on the symmetry of the detector arrangement and the severity of the matrix. In such cases a weighting factor is applied to each measured location to derive a VWA response, since locations at different radii in the drum are representative of cylindrically symmetric regions of different volumes. For a large box, rotation during the assay is not practical and one may, for example, choose to map the response characteristics across only one quadrant of the box in detail. To this end, a subset of points covering the whole box volume would be selected on, for example, a $7 \times 7 \times 4$ grid of rectangular voxel elements, invoking symmetry arguments for the chamber walls and detector configuration, to infer the response for other locations. Even so, this represents a major task consuming both time and people resources.

\subsection{Matrix Compensation}

In section 7.9, Calibration Methodology, we described the various options for calibration of the DDA system, both for fixed matrix types and for a general, unknown matrix. In the case of a known, fixed matrix, the measurement accuracy and hence the Total Measurement Uncertainty 


\begin{tabular}{|l|l|r|}
\hline & Canberra Industries, ACK\# D0043 & 33296 B \\
\hline Created: 29-September-2000 & IBIS Project & Revision B \\
\hline Revised: 13-January-2003 & Preliminary Design Study & Page 80 of 156 \\
\hline
\end{tabular}

(TMU) is determined by the variation of the response with the fissile material position inside the box. In this case the scanning pattern of irradiation by the pulsed $14 \mathrm{MeV}$ neutron generator is very important. For example a scheme in which the box is irradiated on both sides, by rotating it through 180 degrees between the two measurements, can eliminate the large near / far flux ratio which would otherwise lead to very high spatial variations. Another important aspect of achieving a flat spatial response profile is to arrange for the system to be somewhat undermoderated in the sense that fast neutrons are injected into the box and generate a thermal flux inside it. A little bit of moderator in the box results in the self-generating thermal flux having a spatial profile that partially balances the efficiency. By treating the matrix as an integral part of the system, 'typical' matrices can be placed in a region of the operating range for which matrix dependencies are reduced.

In the most general case where the box matrix is unknown, then the options for matrix compensation must be explored. The matrix compensation approach in the IBIS system draws upon the experience from a wide range of systems, to provide the maximum amount of information possible on the matrix composition. This ensures that the most accurate matrix compensation can applied, provided that the data analysis algorithms have been developed to an appropriate level. Our approach facilitates a determination of the spatial variability of the matrix properties.

Traditional drum - sized DDA systems in the United States use a single "cavity flux monitor" (CFM) and "barrel flux monitor" (BFM) pair (usually small "pencil" ${ }^{3} \mathrm{He}$ proportional counters), to provide the matrix compensation. Briefly, the cavity flux monitor provides a normalization to both the absolute neutron output of the pulsed $14 \mathrm{MeV}$ neutron generator, and the absolute thermal neutron interrogating flux. The BFM is closely coupled to the drum surface; it is effectively collimated by using a cadmium "blinker" so that it samples only the thermal neutron flux inside the drum. The die-away time in the BFM provides an index for the matrix absorption properties of the matrix, while the "zero time" extrapolated count rate provides a matrix moderation index. Moderating matrices act to thermalize a greater proportion of the available neutron flux, and hence increase the thermal neutron flux which is built up a short time $(\approx$ few hundred $\mu$ s) after the $14 \mathrm{MeV}$ neutron burst. Absorbing matrices then act to shorten the die-away time of the decaying flux profile, and reduce the integrated thermal neutron flux over the DDA counting interval. Generally, the CFM and the BFM are located in corners of the assay chamber, positioned roughly at the mid height of the drum, to provide the most effective drum - averaged sampling. In the case of the CTEN instrument [48] imaging techniques have been developed.

For the Passive mode assay, a scanning "Add-A-Source" system will be implemented, to provide a matrix compensation for the passive neutron coincidence assay. Briefly, a small ${ }^{252} \mathrm{Cf}$ source is introduced to the outer surface of the sample container, where it interrogates the drum. The additional Totals and Reals coincidence count rates observed in the detectors, is correlated to the specific PNCC calibration parameter for Pu inside the container, using an empirical relationship or "look-up tables". Counting in Totals mode, a correction may be obtained for the effect of the matrix on the detection efficiency for fission neutrons. By counting in Reals mode, information is 


\begin{tabular}{|l|l|r|}
\hline & Canberra Industries, ACK\# D0043 & 33296 B \\
\hline Created: 29-September-2000 & IBIS Project & Revision B \\
\hline Revised: 13-January-2003 & Preliminary Design Study & Page 81 of 156 \\
\hline
\end{tabular}

also obtained on the effect of the matrix on the fraction of coincidence events which are observable inside the fixed coincidence gate.

For the IBIS system, we will investigate the potential benefit from combining the AAS results with the DDA matrix monitor information, with a view to investigating whether the matrix compensation performance can be enhanced - particularly in regard to active efficiency (or "transmission") correction.

In addition, it is intended to use the results of the neutron imaging analysis, to further enhance the results of the DDA assay, by coupling this data with the spatial information obtained from the matrix compensation monitors.

An important physics aspect of the matrix compensation is the limited spatial resolution that can be achieved. In the usual arrangement where one employs only a single CFM / BFM pair, it is necessary both to infer a global average matrix compensation, and to assume that the matrix is indeed homogeneous. A well known difficulty with this approach, is that the BFM is sensitive only to the matrix material in its vicinity. For example, if a drum contains two discrete matrix layers with greatly differing neutronic properties, a barrel flux monitor on the underside of the drum will be sensitive only to the matrix material on the lower half of the drum. By deploying an array of (BFM) matrix monitors located at different positions on the outer surface of the sample container, the spatial variation of the matrix properties can be measured. For example, two matrix monitors sampling the top and bottom half of a drum respectively, can greatly improve the assay accuracy. This is further enhanced if the matrix monitor information is coupled with the neutron imaging results, as the matrix compensation factors derived from the two matrix monitors can be weighted by the relative fissile masses in the two layers of the drum.

An attempt can be made to deal with layered matrices, by deploying an array of matrix monitors (we shall use the term "matrix monitors" from now on, in place of "Barrel Flux Monitors" as our discussion is no longer restricted to barrel containers) located and collimated to view different axial sections of a container. In this way, the monitors may be used both to provide an indication, or warning, of the presence of discrete matrix layers, and ultimately to improve the performance of the matrix compensation algorithm by providing separate compensation factors. While the monitors can be collimated so that they are sensitive only to thermal neutrons emerging from the drum within a narrow entrance cone, there is a limit to the sensitivity which can be achieved, such that no benefit is realized from incorporating more than a certain number of monitors. This optimum number is determined by the thermal neutron transport properties of the various matrix layers.

From the subsequent discussion in the context of our over arching goal of trying to obtain neutron emission images with a spatial resolution of the order of one foot, we propose to use six pairs of matrix monitors. Each pair is a fast (Cd wrapped), thermal (bare), polyethylene wrapped ${ }^{3} \mathrm{He}$ filled proportional counter. The pairs will be arranged in two columns of three. Each proportional counter will have its own preamplifier/amplifier/discriminator and be fed into a 


\begin{tabular}{|l|l|r|}
\hline & Canberra Industries, ACK\# D0043 & 33296 B \\
\hline Created: 29-September-2000 & IBIS Project & Revision B \\
\hline Revised: 13-January-2003 & Preliminary Design Study & Page 82 of 156 \\
\hline
\end{tabular}

separate channel of a PATRM/PCI data acquisition module. Analysis of the list mode data allows the magnitude and temporal profiles of the monitors to be quantified. This forms the basis of the matrix compensation scheme and in addition will help gauge the matrix fill height in the crate. The monitors are likely to be placed on the cavity wall opposite to the neutron generator, but final selection will be guided by experiment.

By forming a crude grid it is hoped that the array of matrix monitors will provide coarse spatial information about the neutronic properties of the matrix. This can be modulated using the density profile obtained from the $\gamma$-ray transmission measurement in order to generate an improved set of response elements for each voxel. This is needed to help improve the quality of the neutron imaging. The matrix fill height can also be verified by the $\gamma$-ray transmission data.

\subsection{Experimental Experience with Matrix Monitor Resolution}

A set of DDA measurements has been made [Chard, Croft, and Sharp, 2000] with layered matrices in $200 l$ drums, in order to test the limits of spatial resolution which can be achieved using an array of matrix monitors deployed up the side of the drum. We now summarize this work, with a view towards using the results to provide some insight in to the maximum number of matrix monitors which will be useful for the IBIS neutron assay chamber.

"Bi - matrices" were used for this study, the bottom $\approx 1 / 2$ of the drum being filled with one homogeneous matrix and the top $\approx 1 / 2$ filled with a second homogeneous matrix. In practice, the sharpness of the transition was limited to approximately $50-100 \mathrm{~mm}$, by the practical difficulty of ensuring uniform packing. $\mathrm{Bi}$ - matrices were formed using combinations of polyethylene chips (bulk density $\left.0.43 \mathrm{~g} . \mathrm{cm}^{-3}\right)$, sand $\left(1.39 \mathrm{~g} . \mathrm{cm}^{-3}\right)$, and iron shot in polyethylene bottles $(1.33$ g. $\left.\mathrm{cm}^{-3}\right)$.

The matrix compensation methodology described in [Armitage and Sherwood, 1987] was used as the basis for the matrix compensation measurements. Matrix monitor measurements of the lifetime correction factor, $\mathrm{C}$ were made[Chard, Croft, and Sharp, 2000]. Equation 19 provides a definition of this quantity, which gives a classification of the matrix absorption. In Equation 19 , $\mathrm{MM}(\mathrm{t})$ represents the count rate in the matrix monitor as a function of time $(\mathrm{t})$ following the 14 $\mathrm{MeV}$ burst. A cadmium shielded matrix monitor was placed at a number of heights on the outer surface of the drum. We are hence attempting to derive an axial scan of the thermal neutron absorption properties in a drum. The results clearly indicate that $\mathrm{C}$ levels out to a particular distinct value for each layer, with a continuous transition between layers. The spatial profile for an example "Tri - matrix" comprising 3 matrix layers (poly chips, iron shot and void) is shown in Figure 16. 


$\mid$\begin{tabular}{|l|l|r|}
\hline & Canberra Industries, ACK\# D0043 & 33296 B \\
\hline Created: 29-September-2000 & IBIS Project & Revision B \\
\hline Revised: 13-January-2003 & Preliminary Design Study & Page 83 of 156 \\
\hline
\end{tabular}

$$
C=\frac{\int_{t=0}^{10 m s} M M(t)}{\int_{t=0.7}^{10 m s} M M(t)}
$$

The characteristic length of the transition across two layers, $l$, depends on both the physical sharpness of the interface, $(\approx 50-100 \mathrm{~mm})$ and the neutronic transport properties of the respective matrix materials. The quantity $l$, was estimated for each bi - matrix by fitting a curve to the matrix monitor data, defining $l$ for each bi - matrix component as the distance between the center of the physical transition to the point at which the response reaches a plateau. The results indicate typical values in the range $50-200 \mathrm{~mm}$. In an attempt to correlate this with the neutronic properties of the materials, an assessment has been made of the thermal neutron migration length, $r$, in each of the matrices considered here. This depends upon the macroscopic thermal neutron absorption cross-section, $\Sigma_{\mathrm{a}}$, the total macroscopic scattering cross - section, $\Sigma_{\mathrm{s}}$, and the average cosine of the scattering angle per collision (in the laboratory frame of reference), $\bar{\mu}$, according to Equation 20 (derived from [Glasstone and Edlund, 1959; Littler and Raffle, 1957]). The results of this assessment are summarized in Table 9. It is clear that $l$ and $r$ are in broad agreement, although the finite physical sharpness is inevitably an additional contributory factor such that one would expect $l$ to be slightly greater than $r$. The migration length can be considered to impose a fundamental limit on the sensitivity of a multiple matrix monitor approach to monitoring layered matrices. It can also be considered to be an indication of how the response to fissile material varies across a layered matrix transition. That is, the fissile material and matrix monitor response are expected to exhibit the same behavior. One can visualize what is happening by imagining the path that a neutron takes from its point of origin to its point of detection. The path is not a simple straight line but a random walk. A projection along the last segment of its path does not point to its point of origin. In this sense the image is "bloomed". There would hence be no benefit trying to achieve a better spatial resolution by making measurements at a finer resolution than this.

$$
r=\sqrt{\frac{2}{\sum_{a} \Sigma_{s}(1-\bar{\mu})}}
$$

Eq. 20

In a real assay chamber, it is necessary to carefully consider the optimum number of matrix monitors. The thermal neutron flux poisoning from the cadmium collimators of an unnecessarily large array of detectors, may be prohibitive. It is unlikely that more than say four discrete layers will occur in a real waste drum in which case four evenly spaced matrix monitors should provide reasonable matrix compensation. The greater the number of discrete layers, the greater is the extent of the "smearing" due to the layer transitions, such that there is no benefit in terms of matrix compensation performance from incorporating a larger number of matrix monitors. 


\begin{tabular}{|l|l|r|}
\hline & Canberra Industries, ACK\# D0043 & 33296 B \\
\hline Created: 29-September-2000 & IBIS Project & Revision B \\
\hline Revised: 13-January-2003 & Preliminary Design Study & Page 84 of 156 \\
\hline
\end{tabular}

We have hence demonstrated the limitations in the ability of multiple matrix monitors for monitoring discrete layered matrices, by experiment. The response varies continuously across the transitions on scales of approximately $100-250 \mathrm{~mm}$, broadly consistent with the calculated thermal neutron migration lengths in the various materials. These transition lengths suggest that there is a fundamental upper limit to the number of matrix monitors which is useful.

In the context of the IBIS crate monitor, we can use these results to give some guidance as to the appropriate voxel size into which the box can be considered to be split, with respect to both spatial matrix mapping and neutron imaging calculations / measurements. The above measured transition lengths $(\approx 100-250 \mathrm{~mm})$ imply that in the context of matrix compensation there would be little benefit in having voxels any smaller than this. Dimensions of approximately 300 $\times 300 \times 300 \mathrm{~mm}$ would therefore seem to be appropriate, the box being split conceptually into say $4 \times 4 \times 7$ voxels ( $h \times w \times l)$. If the voxels were smaller than this, then the matrix properties would tend to "smear" across adjacent voxels. A fixed matrix monitor collimated to view a particular, discrete voxel, would give results indicative of some combination of the voxel and its nearest neighbors.

Neutron imaging relies on the detection of the prompt fission neutrons which arise either from spontaneous fission (for passive mode assay), or thermal neutron induced fission events (for active mode assay). At their birth inside the waste container, the neutrons are fast $(1-2 \mathrm{MeV})$, and their detection relies on the their thermalization within the FNDP's. For non - moderating matrices, a large fraction of the fission neutrons may travel through the container without suffering any collisions with the matrix material. For example, the total (that is, elastic + inelastic) microscopic elastic scattering cross-section for ${ }^{56} \mathrm{Fe}$ at $1 \mathrm{MeV}$ is $\approx 2.6$ barns, which translates to a macroscopic cross-section of $0.0139 \mathrm{~cm}^{-1}$ for an iron density of $0.5 \mathrm{~g} . \mathrm{cm}^{-3}$. This corresponds to a mean free path of $719 \mathrm{~mm}$, which is of the order of half the box dimensions (width or height). The fast neutrons exiting the container will therefore largely retain their localization to the source position. However, scattering in the chamber walls and the FNDP moderator provide additional "smearing". The mean free path in graphite is $\approx 50 \mathrm{~mm}$, while that in high density polyethylene (in the FNDP's) is only $\approx 4 \mathrm{~mm}$. Since the graphite wall thickness is at least $150 \mathrm{~mm}$, the spatial resolution for the neutron imaging is expected to be considerably poorer than the FNDP dimensions. The spatial resolution achievable with the neutron imaging technique, is hence unlikely to be any better than that determined above, for the matrix compensation.

In practice, of course, one can only monitor the matrix properties for the "near - surface" voxels. The optimum arrangement of fixed matrix monitors depends on the pulsed neutron scanning mechanism, and the packing arrangement for material inside the box. If two assays are performed, rotating the box through 180 degrees between measurements, then a fixed set of monitors on one side of the box automatically provides a scan on both sides. In theory, a grid of 4 $\times 7(\mathrm{~h} \times \mathrm{l})$ monitors provides the most useful information if it is likely that the matrix material will vary significantly from one voxel to the next in the $4 \times 7$ grid. Implementation of such a large array for a prototypical instrument is not attractive on cost grounds as each channel of data 


\begin{tabular}{|l|l|r|}
\hline & Canberra Industries, ACK\# D0043 & 33296 B \\
\hline Created: 29-September-2000 & IBIS Project & Revision B \\
\hline Revised: 13-January-2003 & Preliminary Design Study & Page 85 of 156 \\
\hline
\end{tabular}

also requires signal handling nucleonics. Thus a more modest reconfigurable array of matrix monitors is envisaged for the IBIS system, comprising of the order of 6 matrix monitor pairs in total.

Table 9. Comparison of measured transition lengths $(l)$ and calculated thermal neutron migration lengths for layered matrix components. For each bi - matrix component, $l$ has been averaged over the results with different "partner bi - matrices".

\begin{tabular}{|c|c|c|}
\hline $\begin{array}{c}\text { Bi - matrix } \\
\text { Component }\end{array}$ & $\begin{array}{c}\text { Approximate measured } \\
\text { Transition length } \\
l(\mathrm{~mm})\end{array}$ & $\begin{array}{c}\text { Calculated thermal neutron } \\
\text { migration length } \\
r(\mathrm{~mm})\end{array}$ \\
\hline Poly chips & $40 \pm 20$ & 127 \\
\hline Sand & $280 \pm 50$ & 312 \\
\hline Iron / Bottles & $140 \pm 60$ & 141 \\
\hline Empty & $120 \pm 30$ & - \\
\hline (Iron + Water) Mixture & $50 \pm 30$ & 160 \\
\hline
\end{tabular}

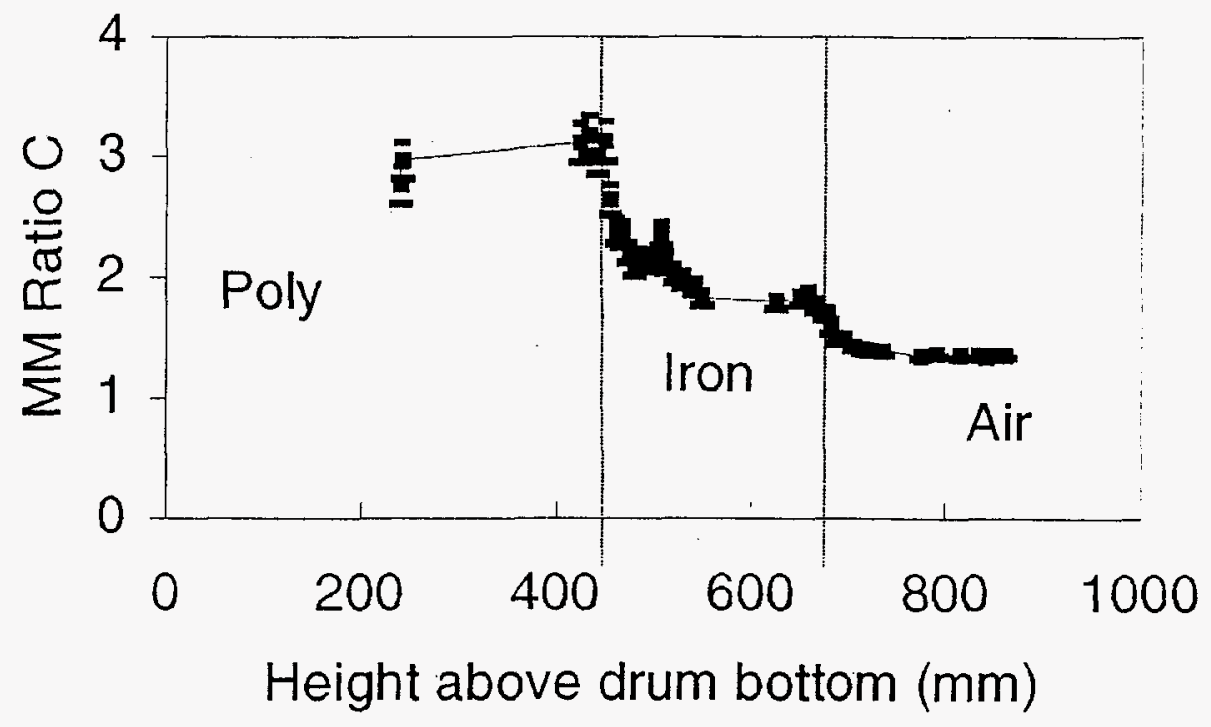

Figure 16. Results of MM measurements in a "Tri - matrix" of 3 layers comprising poly chips, iron shot and air. The physical transitions are marked by dotted lines. 


\begin{tabular}{|l|l|r|}
\hline & Canberra Industries, ACK\# D0043 & 33296 B \\
\hline Created: 29-September-2000 & IBIS Project & Revision B \\
\hline Revised: 13-January-2003 & Preliminary Design Study & Page 86 of 156 \\
\hline
\end{tabular}

\section{Brief Note on LANL MCNP ${ }^{\text {TM }}$ Design Calculations}

\subsection{Introduction}

MCNPTM models described in this section were created in support of the neutron portion of the IBIS system. Preliminary design specifications were provided by Canberra. Both active and passive assays were simulated and benchmarked using data taken for the calibration of the CWAM system. This is similar in physical size and construction to IBIS and therefore provides a good starting position for detailed design study. Calculations were performed using the elemental compositions of the dry combustibles, wet combustibles, scrap metal, polyethylene, and empty B- 25 crates (the same matrices used in the CWAM calibration measurements). The assay chamber size was chosen to accommodate the $4^{\prime} \times 4^{\prime} \times 7^{\prime}$ metal crates used at the Idaho site. Increasing the cavity to accommodate boxes $5^{\prime} \times 8^{\prime} \times 8^{\prime}$ is not seen to be problematic. Once the need is established the design of engineering tasks are straightforward.

\subsection{Design Goals}

As was in the case of CWAM, design goals for BSNS (Box Scanning Neutron System) were directed at correcting deficiencies in its predecessor. To improve accuracy in the original CWAM, efforts were directed at reducing spatial variation of the fissile signal. This was accomplished by placing the neutron generator on a motorized track, allowing interrogation from several positions within the assay chamber. The most obvious enhancement of the BSNS over the original CWAM is the ability to acquire and analyze position sensitive data using the PATRM/PCI module and a mix of horizontal and vertical detector packages. The ability to correct for non-uniform source position coupled with the multiple interrogation positions is expected to improve accuracy in a wide range of matrices.

As with all projects of this magnitude, a combination of physics and cost went into the design for the new system. Components that affect neutron population during an assay such as the specifications of the moderating cavity and detector packages were based on Monte Carlo calculations. The figure of merit used in comparing alternative MCNPTM designs was the product of the thermal flux and detector efficiency. Maximizing the product of these two quantities maximizes active sensitivity. Detection limits in both the active and passive modes are expected to be comparable or exceed those of CWAM as measured in the surrogate matrices during the calibration phase. For uranium, this limit corresponds to $35 \mathrm{pCi} / \mathrm{g}$ total activity thus allowing quantification at the LLW/Sanitary waste cutoff. For plutonium, active sensitivity is expected to be approximately $10 \mathrm{nCi} / \mathrm{g}$ alpha activity, allowing quantification at both the TRU and LLW levels.

Although accuracy and sensitivity goals have remained the same throughout the design phase, the transportability of BSNS has changed due to recent feedback from potential customers.

Originally, the structural design needed only to be modular and easy to assemble/disassemble. To reduce material cost, the amount of graphite used in the chamber walls was constrained to less than 4 inches. To maintain the same active sensitivity with less graphite, a moderating assembly 


\begin{tabular}{|l|l|r|}
\hline & Canberra Industries, ACK\# D0043 & 33296 B \\
\hline Created: 29-September-2000 & IBIS Project & Revision B \\
\hline Revised: 13-January-2003 & Preliminary Design Study & Page 87 of 156 \\
\hline
\end{tabular}

surrounding the neutron generator was necessary. Reducing the amount of graphite between the assay chamber and the detector packages also improved the passive sensitivity. Based on these considerations, a variety of neutronic design options were determined.

With the recent decision to install BSNS on a standard trailer suitable for transportation on public highways, these design options need to be revisited. Although designs presented will fit on a standard trailer, the approximate system weight is near the DOT limit. Further calculations are therefore being conducted to reduce the system footprint without sacrificing active sensitivity.

\subsection{Monte Carlo Design Calculations}

The analysis of neutron slowing down in a differential die-away assay is intrinsically time and energy dependent. The average energy of a neutron pulse decreases continuously until thermal equilibrium is reached with the matrix material. For a large waste container containing a number of different regions with distinct nuclear properties, the neutron distribution is space-dependent as well. In terms of data acquisition in the scaler mode, the continuous time-energy distribution is sampled at multiple time windows and for two broad energy groups.

The differentiation between epithermal and thermal energy groups is accomplished through the use of bare and shielded ${ }^{3} \mathrm{He}$ proportional detectors. Small ${ }^{3} \mathrm{He}$ proportional counters are used to measure the intensity of the thermal interrogating flux. Thermal flux die-away times (inverse of the fundamental decay constant) can be calculated using timing data from the list mode. Ratios of responses from bare and shielded detectors and different time windows can be used to measure the moderating ability and thus the neutron slowing down time of different matrix materials.

Monte Carlo techniques were used to simulate the active assay by following the pulse of neutrons from the 14-MeV generator through slowing down, thermalization, and diffusion processes in the matrix materials. From the simulation of an active assay, the interrogating neutron intensity and energy spectra were calculated as a function of space and time for the surrogate matrices used in the CWAM calibration. A Monte Carlo calculation consists of simulating a finite number of neutron histories from which an average or collective behavior such as flux or current density can be deferred. In mathematical terms, this collective behavior is the mean or expected value of some quantity $\mathrm{x}$. For the system design, the neutron flux density for the active mode and the ${ }^{3} \mathrm{He}(\mathrm{n}, \mathrm{p})^{3} \mathrm{H}$ reaction for the passive mode were the primary tallies.

MCNPTM is a general-purpose, continuous energy, time-dependent Monte Carlo N-Particle code that can be used for neutron, photon, or electron transport through three-dimensional configurations. The geometry features in MCNPTM allow a three-dimensional model to be constructed mathematically of cells containing user-specified materials. During the design phase of an assay system, geometric parameters are varied to determine the best active or passive performance within the given design constraints. 


\begin{tabular}{|l|l|r|}
\hline & Canberra Industries, ACK\# D0043 & 33296 B \\
\hline Created: 29-September-2000 & IBIS Project & Revision B \\
\hline Revised: 13-January-2003 & Preliminary Design Study & Page 88 of 156 \\
\hline
\end{tabular}

In the interest of maximum flexibility, the generation of $\mathrm{MCNP}^{\mathrm{TM}}$ input files was automated to allow both system design and matrix/container parameters to be varied easily. Wall thickness, wall materials, detector package energy filters, number of detector tubes per package, number of packages, orientation of detector packages, detector lengths, and detector atmospheres can be varied in both passive and active simulations. The input file generation program allows different types of containers and matrix materials. Tallies in the active mode such as flux or induced fissile signal can be integrated over the entire crate or in easily varied voxel sizes. Detection efficiency can be calculated using either a ${ }^{240} \mathrm{Pu}$ or ${ }^{252} \mathrm{Cf}$ source uniformly dispersed throughout a matrix or configured as a point source located at any position within the matrix. Neutron flux intensities and/or energy spectra can be calculated as a function of fine time bins simulating list mode (dieaway time) or broad time bins (scaler-defined time windows).

To process the large amount of data generated, visual basic macros were written to read MCNPTM output files into an EXCEL spreadsheet and tabulate relevant information. Passive data was tabulated by detection efficiency as a function of matrix, source position, detector dieaway times, and by individual detector banks. Active data was organized similarly for the interrogating flux intensities as a function of matrix, voxel (currently 96-1' x 1' x 1' voxels), and time intervals. Neutron energy spectra have also been calculated as a function of matrix type and position. In addition to the traditional passive and active assay simulations, dose calculations were also performed as a function of neutron generator position for each of the proposed system designs.

Although this discussion focuses on the use of MCNPTM simulations in system design optimization, the EXCEL-Visual BASIC programs were also developed to allow matrix/container parameters to be varied easily during the calibration phase. The excessive effort required in measuring point-response data for a passive-active system as large the CWAM system crate dictates the use of Monte Carlo simulated data to extend the range of applicable matrices. A large number of matrices will still need to be measured during the calibration phase to develop matrix and calibration algorithms. This data can then be used to benchmark the asbuilt MCNPTM model thus providing a tool to generate additional data points for interpolation with respect to the measured matrix data set.

\subsubsection{Benchmark Model with CWAM Calibration Data}

The EXCEL-Visual BASIC programs were completed by the end of June with the first MCNPrM model being that of the original CWAM. Data from the CWAM calibration work was used to benchmark both efficiency and neutron flux MCNPTM -calculated data. All calculations were performed using the elemental compositions of the dry combustibles, wet combustibles, scrap metal, polyethylene, and empty B-25 crates (the same matrices used in the CWAM calibration measurements). To date, only B-25 crates ( $\left.4^{\prime} \times 4^{\prime} \times 6^{\prime}\right)$ have been modeled but other size crates will be modeled in the future.

The MCNPTM system geometry was verified by comparing the measured detection efficiency and dieaway time with calculated values for an empty crate. MCNPTM detector tallies were 


\begin{tabular}{|l|l|r|}
\hline & Canberra Industries, ACK\# D0043 & 33296 B \\
\hline Created: 29-September-2000 & IBIS Project & Revision B \\
\hline Revised: 13-January-2003 & Preliminary Design Study & Page 89 of 156 \\
\hline
\end{tabular}

constructed to allow the calculation of the detector efficiency using both ${ }^{240} \mathrm{Pu}$ point sources at various locations or crate-average uniform source distributions. The measured efficiency for the CWAM system for a ${ }^{240} \mathrm{Pu}$ point source centered in an empty crate is $13.7 \%$ compared to a MCNPTM -calculated value of $14.2 \%$. The ${ }^{3} \mathrm{He}(n, p)^{3} \mathrm{H}$ reaction rate was also tabulated in a series of narrow time bins from 0 to $300 \mu \mathrm{s}$. Figure 17 shows the MCNPTM TM-calculated time distribution for the sum of the detector packages (system totals). The MCNPTM -calculated detector die-away time for an empty crate in the CWAM system was $44 \mu$ s compared to a measured value of $42 \mu \mathrm{s}$.

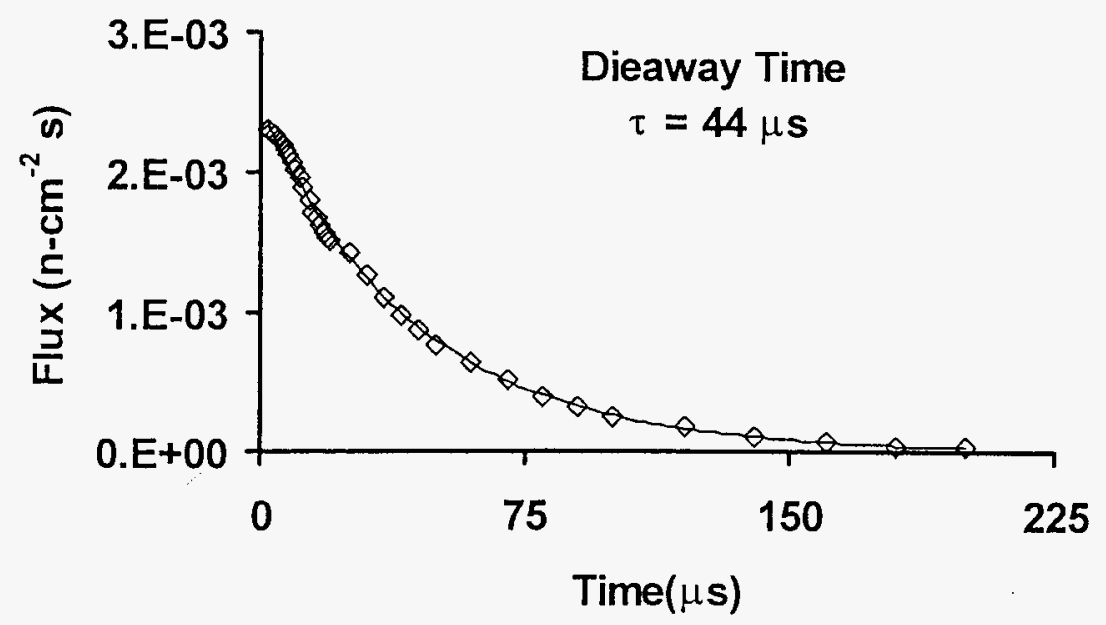

Figure 17. MCNPTM TM-calculated detector die-away time for the CWAM system in an empty crate.

\subsubsection{Wall Optimization}

During the month of July, over 1500 passive and active assay simulations of the MCNPrM model of the new IBIS system were performed in support of the neutronic design. The overall design goal was to increase detection efficiency without reducing significantly the magnitude of the thermal interrogating flux. To assess the various system configurations, the figure of merit used was the multiplicative product of the detection efficiency to a ${ }^{240} \mathrm{Pu}$ source uniformly dispersed in the various matrix materials within a B-25 crate and the integrated thermal flux inside the crate during the time period of 850 to 2,850 microseconds, i.e., the thermal interrogation period.

Initial design calculations focused on reducing the graphite thickness between the detector packages and the assay chamber. A series of wall optimization calculations that included both active and passive simulations, were performed using the basic CWAM design with a slightly larger assay chamber to accommodate crates measuring $4^{\prime} \times 4^{\prime} \times 7^{\prime}$. The inner graphite liner 


\begin{tabular}{|l|l|r|}
\hline & Canberra Industries, ACK\# D0043 & 33296 B \\
\hline Created: 29-September-2000 & IBIS Project & Revision B \\
\hline Revised: 13-January-2003 & Preliminary Design Study & Page 90 of 156 \\
\hline
\end{tabular}

thickness was varied in one-inch increments from the CWAM design of 6 inches down to 1 inch. Since optimal wall thickness is a strong function of matrix material, calculations were performed for the matrix set used in the CWAM calibration.

Figure 18 shows the crate-average thermal flux as a function of graphite thickness for the matrix set. Neutron generator position is in back center of B-25 box. As expected, the interrogating thermal flux increases with increasing graphite thickness. Figure 19 shows the detection efficiency for a uniformly dispersed ${ }^{240} \mathrm{Pu}$ source for the same matrix set as a function of graphite thickness. The CWAM design contains 24 cadmium-shielded detector packages with 4 detectors per package for a total of 96 detectors. All detectors are 5 foot in length with a pressure of 4 atmospheres. Cadmium thickness is 0.060 inches. The graphite is highly purity reactor grade A material.

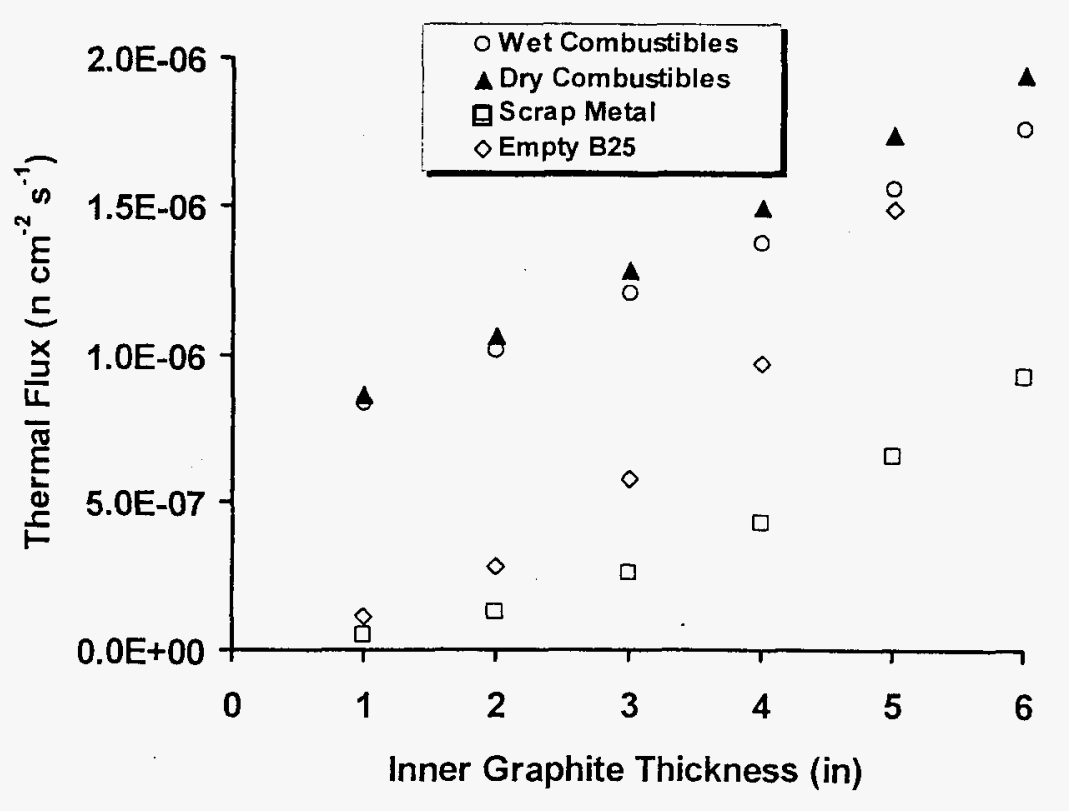

Figure 18. MCNPTM -calculated thermal interrogating flux as a function of graphite thickness. 


\begin{tabular}{|l|l|r|}
\hline & Canberra Industries, ACK\# D0043 & 33296 B \\
\hline Created: 29-September-2000 & IBIS Project & Revision B \\
\hline Revised: 13-January-2003 & Preliminary Design Study & Page 91 of 156 \\
\hline
\end{tabular}

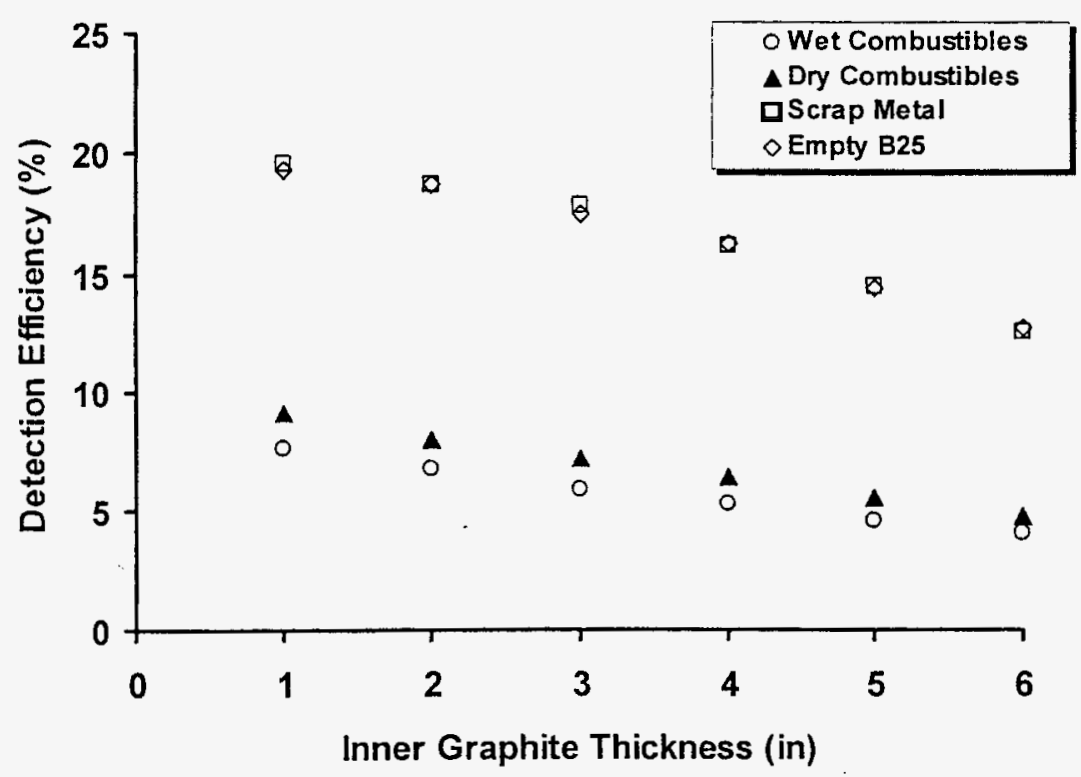

Figure 19. MCNPTM-calculated detection efficiency for a uniformly dispersed ${ }^{240} \mathrm{Pu}$ source as a function of graphite thickness.

Figure 20 shows the figure of merit as a function of graphite thickness. The empty crate is not shown but displays the same trends as the scrap metal matrix, i.e. increasing FOM with increasing inner graphite liner. The CWAM design at 6 inches was optimized for a nonhydrogenous matrix such as scrap metal. A large percentage of the inventory crates at Y-12 were scrap metal and could not be measured by their existing gamma-ray system. 


\begin{tabular}{|l|l|r|}
\hline & Canberra Industries, ACK\# D0043 & 33296 B \\
\hline Created: 29-September-2000 & IBIS Project & Revision B \\
\hline Revised: 13-January-2003 & Preliminary Design Study & Page 92 of 156 \\
\hline
\end{tabular}

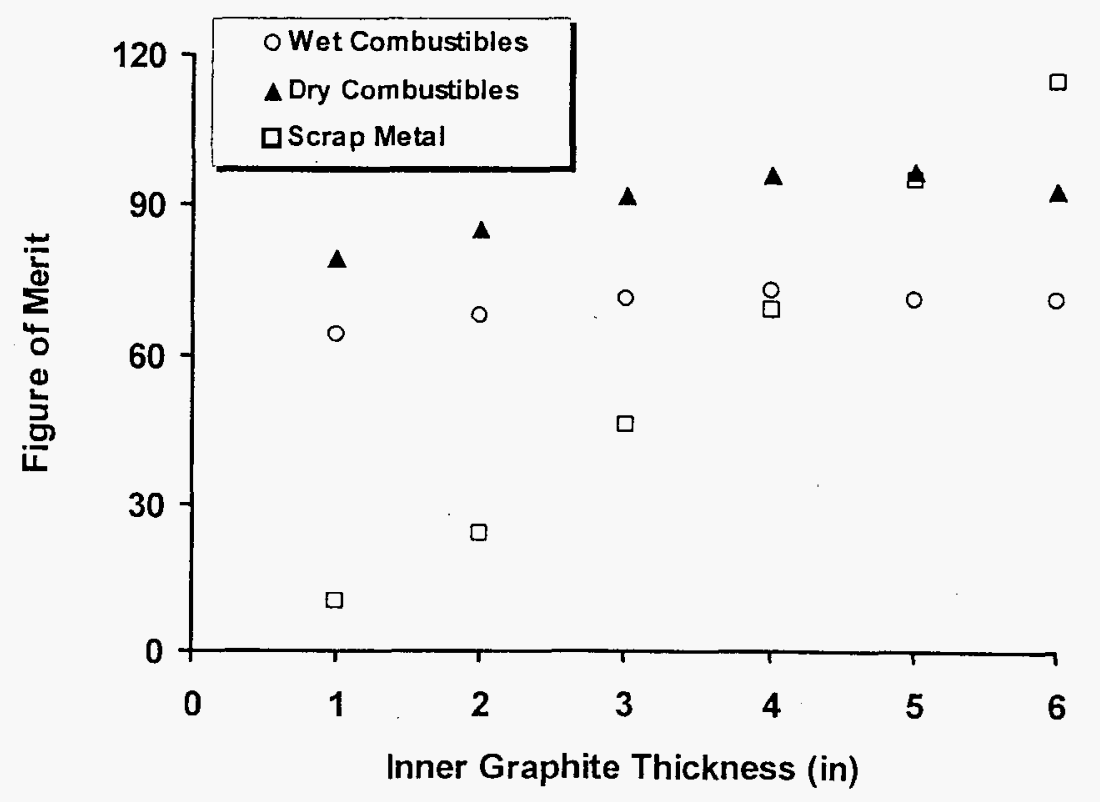

Figure 20. MCNPTM -calculated Figure of Merit (FOM) as a function of graphite thickness.

\subsubsection{Moderating Assembly Configuration}

To maintain the thermal interrogating flux equal to or better than the original CWAM system with less graphite, a variety of moderating assemblies, i.e., materials surrounding the neutron generator, were simulated to "boost" the neutron output. Since a stated goal was that the graphite wall thickness should measure no more than 4 inches, these simulations used the 4-inch thickness. The moderating assembly consisted of combinations of tungsten or lead to taken advantage of the $(n, 2 n)$ reactions and carbon or beryllium configured as concentric cylinders around the neutron generator. From experimental data taken during the CWAM calibration, the optimal neutron positions were determined to be located at the back lower third of the crate. Therefore, total moderating assembly thickness in these simulations was constrained by the extra space available in the back of the assay chamber.

Figure 21 shows the figure of merit for 20 different moderating assemblies for the empty and scrap metal matrices. The straight lines are the figure of merit for the original CWAM system. To achieve an active sensitivity better than or equal to CWAM, the FOM in each of the matrices must be greater than that achieved in the CWAM system. Figure 22 shows the FOM for the same moderating assemblies in the dry and wet combustibles matrices. As expected, moderating assembly performance is dependent on the hydrogen content of the matrix. Of the moderating assemblies tested, the combination of $1.5 \mathrm{~cm}$ of tungsten surrounded by $6 \mathrm{~cm}$ of beryllium gave an active sensitivity greater than CWAM in all matrices. 


\begin{tabular}{|l|l|r|}
\hline & Canberra Industries, ACK\# D0043 & 33296 B \\
\hline Created: 29-September-2000 & IBIS Project & Revision B \\
\hline Revised: 13-January-2003 & Preliminary Design Study & Page 93 of 156 \\
\hline
\end{tabular}

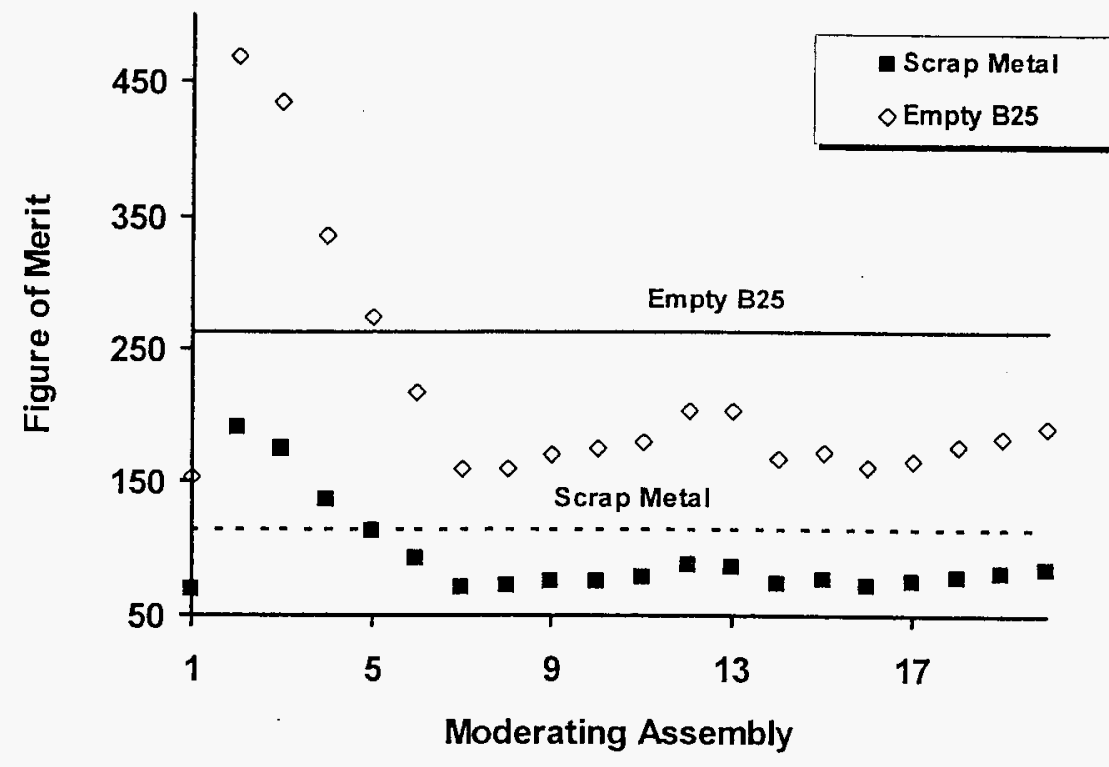

Figure 21. MCNP'M -calculated Figure of Merit (FOM) for 20 different moderating assemblies in the empty and scrap metal matrices.

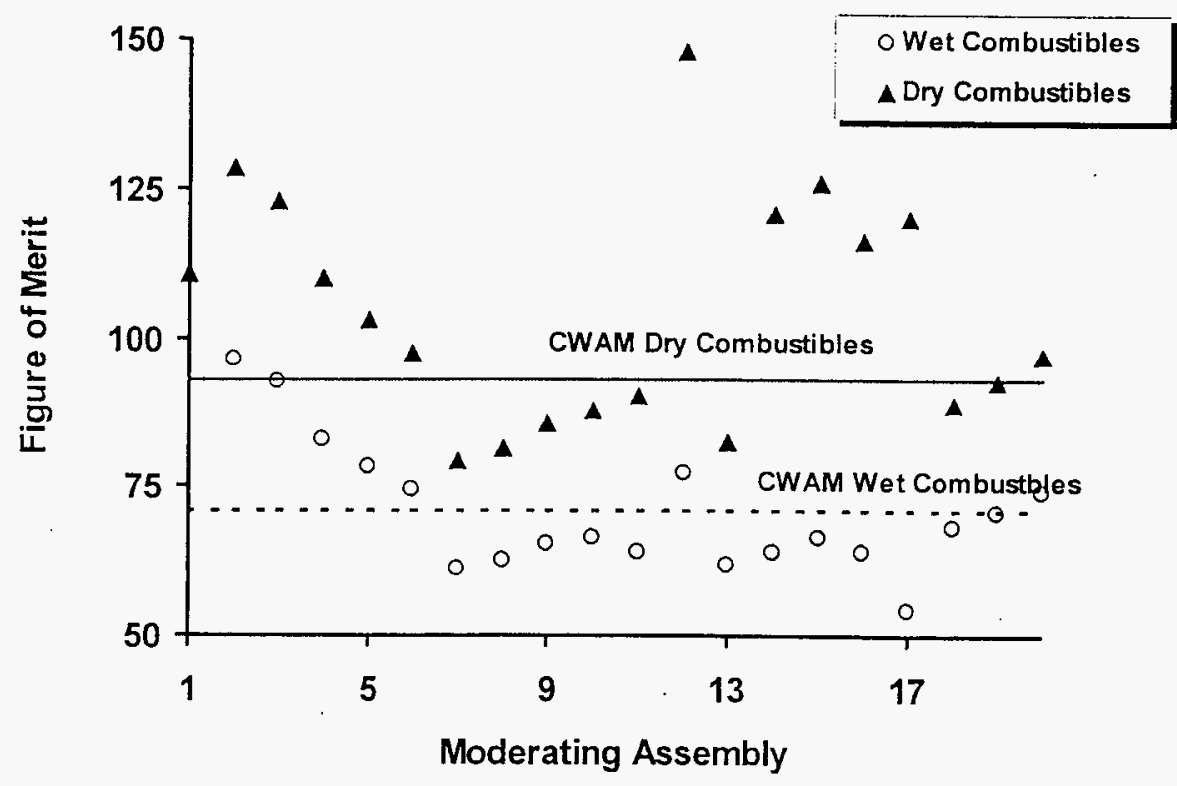

Figure 22. MCNPTM - calculated Figure of Merit (FOM) for 20 different moderating assemblies in the dry and wet combustibles matrices. 


\begin{tabular}{|l|l|r|}
\hline & Canberra Industries, ACK\# D0043 & 33296 B \\
\hline Created: 29-September-2000 & IBIS Project & Revision B \\
\hline Revised: 13-January-2003 & Preliminary Design Study & Page 94 of 156 \\
\hline
\end{tabular}

The purpose of the moderating assembly simulations at this point was determine if the use of a moderating assembly would offset the loss of graphite in the chamber walls. Although the simulations were performed with the moderating assembly inside the assay chamber, structurally it could be embedded in the back wall. Further design calculations will be performed to optimize the moderating assembly during the mechanical and structural design phase. Typically, moderating assemblies used in DDT systems are many centimeters thick resulting in a completely thermal interrogating flux. This is not the intention of this application. In designing the final moderating assembly, both the intensity and energy spectra of the interrogating flux as a function of position and matrix will be calculated using MCNPTM. The goal will be to maximize crate-average interrogating flux intensities while maintaining the ability to penetrate to the center of the matrix.

\subsubsection{Detector Package Configuration}

The data presented above uses the same detector configurations as the original CWAM. For a wall thickness of 4 inches, the detection efficiency for a uniformly distributed ${ }^{240} \mathrm{Pu}$ source increases from $12.7 \%$ to $16.2 \%$ for an empty crate. The most obvious way to increase detection efficiency is to add more detector packages. The same set of wall optimization simulations were performed increasing the number of detectors from 96 to 120 . The CWAM detector package configuration with 4 detectors per package at 4 atmospheres and 5-foot active length was used. Simulations were also performed keeping the same 24 detector package configurations of CWAM (96 total detectors) but increasing the tube pressure to 10 atmospheres. Tables 10 and 11 compare the MCNPTM -calculated Figure of Merit and detection efficiency for three detector configurations. Graphite thickness for these comparisons is 4 inches. No moderating assembly is used.

Table 10. MCNPrM -calculated Figure of Merit with a 4-inch graphite liner for three detector configurations.

\begin{tabular}{|l|c|c|c|}
\hline \multicolumn{1}{|c|}{$\begin{array}{c}\text { Surrogate } \\
\text { Matrix }\end{array}$} & $\begin{array}{c}\text { 96 Detectors } \\
\text { 4 Atm. }\end{array}$ & $\begin{array}{c}\text { 120 Detectors } \\
\text { 4 Atm. }\end{array}$ & $\begin{array}{c}\text { 96 Detectors } \\
\text { 10 Atm. }\end{array}$ \\
\hline Empty B25 & 157 & 140 & 170 \\
\hline Scrap Metal & 69 & 64 & 78 \\
\hline Dry Combustibles & 96 & 103 & 105 \\
\hline Wet Combustibles & 73 & 78 & 79 \\
\hline
\end{tabular}




\begin{tabular}{|l|l|r|}
\hline & Canberra Industries, ACK\# D0043 & 33296 B \\
\hline Created: 29-September-2000 & IBIS Project & Revision B \\
\hline Revised: 13-January-2003 & Preliminary Design Study & Page 95 of 156 \\
\hline
\end{tabular}

Table 11. MCNPTM-calculated detection efficiency (\%) with a 4-inch graphite liner for three detector configurations.

\begin{tabular}{|l|c|c|c|}
\hline \multicolumn{1}{|c|}{$\begin{array}{c}\text { Surrogate } \\
\text { Matrix }\end{array}$} & $\begin{array}{c}\text { 96 Detectors } \\
\text { 4 Atm. }\end{array}$ & $\begin{array}{c}\text { 120 Detectors } \\
\text { 4 Atm. }\end{array}$ & $\begin{array}{c}\text { 96 Detectors } \\
\text { 10 Atm. }\end{array}$ \\
\hline Empty B25 & 16.2 & 18.6 & 17.1 \\
\hline Scrap Metal & 16.1 & 18.5 & 17.3 \\
\hline Dry Combustibles & 6.3 & 7.4 & 7.0 \\
\hline Wet Combustibles & 5.3 & 6.1 & 5.7 \\
\hline
\end{tabular}

To optimize active sensitivity, it is better to keep the same number of detectors and increase the tube pressure. Adding more detector packages increases the amount of cadmium in the system and therefore, depresses the thermal flux. The additional cadmium becomes more important as the graphite thickness is decreased. Coincidence counting in both the active and passive modes is better served by increasing the number of detector packages. Detection efficiency squared (a rough estimate of coincidence efficiency) in an empty crate is increased from $2.6 \%$ to $2.9 \%$ (96$10 \mathrm{~atm})$ and finally $3.5 \%(120-4 \mathrm{~atm}$.). At this graphite thickness, the additional gain from either configuration does not appear to outweigh the cost. As shown in Figure 7, the largest gain in detection efficiency is achieved by reducing the graphite thickness.

In addition to wall optimization, a variety of detector package configurations were also simulated in an effort to reduce thermal neutron leakage of the interrogating flux and achieve a reduced dieaway time. In all, 30 different detector package configurations were modeled. Detector diameter, tube pressure, and active length were kept constant. Dimensions of the side, back, front, and top polyethylene moderating material were varied as well as the type of energy filters used. Different combinations of cadmium and borated rubber were simulated. The presence of borated rubber in conjunction with cadmium did reduce the thermal neutron flux leakage but at the price of reduced thermal flux intensity and detection efficiency.

Table 12 shows both the average time for the detector packages to register zero counts from the neutron generator (the so called extinction time) and detector die-away times. Columns 1 and 2 show values representing the average of all the active simulations, including wall optimization runs, performed using 4 atmosphere detectors. Column 3 shows the average detector die-away times for 10 atmosphere detectors. Extinction time from the neutron burst is greater for nonhydrogenous matrices, approximately $750 \mu$ s before returning to zero, as compared to $590 \mu \mathrm{s}$ in the wet combustible. This is consistent with measurement data in both the CTEN and CWAM systems. Detector dieaway times are essentially constant for variations in either detector package configurations or intervening wall thickness. Even over a wide range of matrices, the detector die-away time varies less than $2 \%$. 


\begin{tabular}{|l|l|r|}
\hline & Canberra Industries, ACK\# D0043 & 33296 B \\
\hline Created: 29-September-2000 & IBIS Project & Revision B \\
\hline Revised: 13-January-2003 & Preliminary Design Study & Page 96 of 156 \\
\hline
\end{tabular}

Table 12. MCNPTM-calculated average detector recovery times and die-away times for the matrix set.

\begin{tabular}{|l|c|c|c|}
\hline \multicolumn{1}{|c|}{$\begin{array}{c}\text { Surrogate } \\
\text { Matrix }\end{array}$} & $\begin{array}{c}\text { Extinctionn } \\
\text { Time } \\
(\mu \mathrm{s})\end{array}$ & $\begin{array}{c}\text { Dieaway Time } \\
(\mu \mathbf{s}) \\
\text { 4 Atm.. }\end{array}$ & $\begin{array}{c}\text { Dieaway Time } \\
(\mu \mathbf{s}) \\
\mathbf{1 0} \mathrm{Atm} . .\end{array}$ \\
\hline Empty B25 & $730 \pm 15$ & $45.2 \pm 2.6$ & $40.3 \pm 1.7$ \\
\hline Scrap Metal & $755 \pm 15$ & $45.2 \pm 2.5$ & $39.8 \pm 1.7$ \\
\hline Dry Combustibles & $610 \pm 15$ & $43.9 \pm 1.9$ & $38.5 \pm 1.0$ \\
\hline Wet Combustibles & $590 \pm 12$ & $43.8 \pm 1.9$ & $38.3 \pm 1.0$ \\
\hline
\end{tabular}

\subsection{Discussion of Results}

A stated goal of the new BSNS system was to reduce the overall footprint compared to that of CWAM. However, no conscious attempt was made to fit the system on a $81 / 2$ foot-wide trailer. Therefore, wall optimization and moderating assembly calculations focused on the 4-inch graphite liner. Calculations were also performed to optimize the outer shielding wall, i.e., the section behind the detector packages. A variety of materials, (polyethylene, graphite, and borated-polyethylene) were varied to determine both the effect on detection efficiency (reflection) and external dose to personnel. Four inches of polyethylene was sufficient keep the posted radiation line within a couple of feet of the system. The inner and outer aluminum shells each have dimensions of 0.5 inches. Using the original design criteria, the total wall thickness was reduced from 16 inches for the CWAM system to 13 for the new BSNS.

To maintain the active sensitivity of the CWAM, a moderating assembly would be required. As in the CWAM system, the neutron generator would ride on a motorized track located in the lower back of the assay chamber. The moderating assembly would be a set of concentric cylinders surrounding the neutron generator. The material selections would be determined from further MCNPTM calculations but it is envisioned that either lead or tungsten would be the inner material with carbon on the outside. The overall diameter of the generator and the assembly would be less than 12 inches.

In performing detector package and wall optimization calculations, the number of detectors per package and number of packages were two of the varied parameters. As in the CWAM system, optimization occurred with a 4-detector package configuration using the same amount of polyethylene surrounding the detectors. As for the energy filters, the amount of cadmium was reduced from 0.060 to 0.040 inches and no borated rubber. The optimal number of detectors was determined to be 96 , i.e. 24 packages at 4 detectors per package. Active length used was 5 foot with a tube pressure of 10 atmospheres. It must be noted here that calculations were only performed for 4 and 10 atmospheres. Further calculations may indicate that a slightly lower tube pressure would be adequate. For operational and economic reasons a fill pressure of 6 atmospheres would be normally chosen for 2 inch diameter ${ }^{3} \mathrm{He}$ tubes. 


\begin{tabular}{|l|l|r|}
\hline & Canberra Industries, ACK\# D0043 & 33296 B \\
\hline Created: 29-September-2000 & IBIS Project & Revision B \\
\hline Revised: 13-January-2003 & Preliminary Design Study & Page 97 of 156 \\
\hline
\end{tabular}

For imaging purposes, the detector package orientation in opposing walls will be at right angles to one another. To accommodate the moderating assembly in the back wall (if placed structurally within the wall instead of in the assay chamber), the detector packages may be horizontal while the packages in the door are vertical. Similar schemes are true for the leftright modules and top/floor modules.

The system as designed above would actually fit on a standard DOT-approved trailer with inside dimensions of 8 feet. Overall weight would be approximately $40,000 \mathrm{lbs}$ for pure materials with an additional 10,000 lbs. for steel reinforcements. DOT weight limit for a trailer this size is $65,000 \mathrm{lbs}$.

Additional MCNPTM calculations will be performed in an effort to further reduce the footprint of the system and overall weight. The spatial variation of the signal in both the passive and active modes will also be studied to ensure both accuracy and sensitivity are not compromised in any new proposed designs. The automation of the MCNPTM input files and Visual-BASIC data processing programs will allow these additional calculations to be performed and analyzed in a timely manner. 


\begin{tabular}{|l|l|r|}
\hline & Canberra Industries, ACK\# D0043 & 33296 B \\
\hline Created: 29-September-2000 & IBIS Project & Revision B \\
\hline Revised: 13-January-2003 & Preliminary Design Study & Page 98 of 156 \\
\hline
\end{tabular}

\section{Self Shielding in Lumps of Fissile Material}

Large particles of fissile material are not usually expected to be encountered in waste but may, none the less, be present. Active and passive gamma-ray techniques run the risk of under reporting the fissile material content (unrevealed fault) if some of the material is in the form of "lumps". A lump is a concentration or contiguous mass of nuclear material that is sufficiently large to affect the measured signal. In active thermal neutron interrogation a significant lump can in principle be as small as a few mg [Cogbill and Swinhoe, 1985; Chard and Croft, 1995, pp 557562]. The origin of the problem is the failure of the interrogating neutron flux to uniformly penetrate the lump. The outer layers absorb the neutrons before they reach the inner regions. This gives rise to an underestimation of the fissile mass because the interior material is, in effect, not visible to the assay system. The degree of neutron penetration depends on the spectrum of neutrons incident on the lump. For this reason it varies with both the composition of the waste matrix and on the position of the lump within the waste container. For instance, East and Becker (1994) report that, for a "Second generation" LANL PAN system, the active assay value from a $1.0 \mathrm{~g}$ Pu metal foil $19 \mathrm{~mm}$ in diameter by $0.2 \mathrm{~mm}$ thick varies from $0.24 \mathrm{~g}$ to $0.32 \mathrm{~g}$, depending on its location in the assay chamber.

Suppose we placed a small piece of $\mathrm{U}$ or $\mathrm{Pu}$ at the center of a spherical shield of high density ( $0.955 \mathrm{~g} / \mathrm{ml}$ ) polyethylene of radius $25-50 \mathrm{~mm}$. Experience tells us that the interrogating flux incident on the sample would be fairly soft (thermalized) providing relatively poor penetration into the lump. This is because an inch or two of polyethylene is an effective moderator. Now imagine a piece of fissile material inside a crate of typical dimensions about $1 \mathrm{~m}$ across. The effective density of polyethylene distributed throughout the box to produce a similar overall degree of moderation to the spherical shield is simply obtained by equating the density thickness products in the two cases. Thus an effective density of $0.05 \mathrm{~g} / \mathrm{ml}$ of hydrogen rich material is intuitively going to result in an interrogating flux that is essentially thermal in character. This hydrogen density is commonly encountered in practice and so the moderation of the interrogating spectrum by the matrix can be anticipated to be a common occurrence. This effect means that rethermalization by the matrix can readily become a problem, even in systems where the primary interrogating flux (that is, unperturbed by the sample matrix) contains a substantial non - thermal (epithermal) component. Because of this the potential under reporting of fissile mass as a result of self-shielding is a common concern. The mean free path of thermal neutrons in HEU or Pu metal is only a fraction of a mm. Thus for lumps of material with dimensions of this order or larger (in extreme cases this equates to material masses in the mg range) the inner regions are shielded by the outer layers and the signal quickly becomes proportional to surface area rather than to volume (which is proportional to the fissile mass). Because boxed waste commonly gives rise to a substantial moderator - thickness products it is difficult in practice to achieve an interrogation spectrum with better penetrating power (i.e. a harder spectrum). It is therefore not possible in practice to make meaningful corrections for self-shielding, using techniques which rely on the existence of a substantial epithermal component in the interrogating flux, in addition to a thermal component (which is required to achieve good sensitivity).

Degree of potential underestimation: 


\begin{tabular}{|l|l|r|}
\hline & Canberra Industries, ACK\# D0043 & 33296 B \\
\hline Created: 29-September-2000 & IBIS Project & Revision B \\
\hline Revised: 13-January-2003 & Preliminary Design Study & Page 99 of 156 \\
\hline
\end{tabular}

If we consider the worst case scenarios, namely HEU metal and weapons grade Pu metal spheres the effects can be quite large. The self-shielding factor (SSF), defined as the ratio of the apparent mass (signal) observed to the true mass present, for a range of lump sizes evaluated under the conditions set out above are summarized in the figure below. These have been calculated for a typical 55 gal Harwell DDA chamber empty drum thermal flux, this is a close approximation to a thermal Maxwellian. HEU and reactor grade Pu metal spheres $\left(93 \%{ }^{235} \mathrm{U}\right.$ and $5.6 \%{ }^{240} \mathrm{Pu}_{\text {eff }}$ respectively), with densities of 19.6 and $18.7 \mathrm{~g} / \mathrm{ml}$ respectively have been assumed.

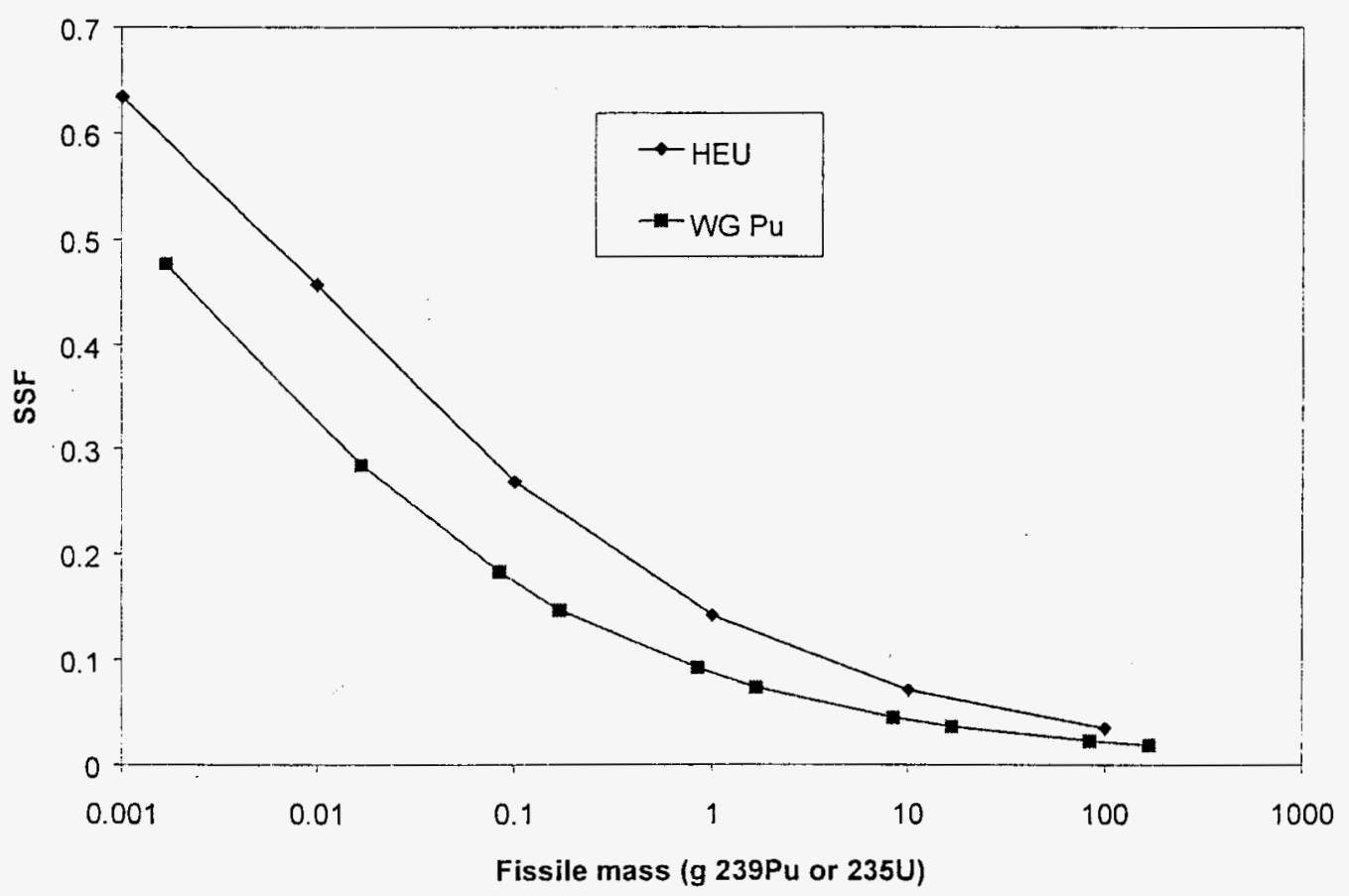

Figure 23. Calculation of self shielding factors for a Cf shuffler flux, for HEU and WG Pu metal spheres.

Self shielding is generally, at a given enrichment, slightly more pronounced for $\mathrm{Pu}$ than for $\mathrm{U}$ due to the higher thermal neutron cross-section of ${ }^{239} \mathrm{Pu}$. In addition the strong resonance in the ${ }^{239} \mathrm{Pu}$ cross section centered at about $0.3 \mathrm{eV}$ means that SSF for epi-thermal neutrons may even be lower than the thermal values.

However, it is widely appreciated (Coop, Nov. 1989) that that the SSFs treated in this fashion are for the worst case scenario, namely for highly enriched metal spheres. Lower enrichment or number density, or a non-spherical shape will result in a less pronounced self shielding effect. For example, it is generally acknowledged that small pieces of $\mathrm{Pu}$ metal in waste will quickly oxidize as a result of contact with moist air to form $\mathrm{PuO}_{2}$ which is more accessible to the interrogating flux, owing to its much lower density.

Dependence on the physical / chemical form: 


\begin{tabular}{|l|l|r|}
\hline & Canberra Industries, ACK\# D0043 & 33296 B \\
\hline Created: 29-September-2000 & IBIS Project & Revision B \\
\hline Revised: 13-January-2003 & Preliminary Design Study & Page 100 of 156 \\
\hline
\end{tabular}

The self shielding factor (SSF) depends upon the physical (i.e. shape) and chemical form of the fissile material, as well as the enrichment. The most severe underestimation thus arises in metallic HEU or weapons grade Pu. For natural or depleted uranium metal, self shielding is generally not significant, equating to an underestimation of no more than a few $\%$ for practical lump sizes of up to several mm typical dimensions.

$\mathrm{Pu}$ is almost the universal solvent and so it comes in a variety of compounds. Metal will be present in DOE wastes although small pieces will have had a tendency to have oxidized, such that significant lumps are unlikely to hold together. $\mathrm{Pu}$ metal and $\mathrm{PuO}_{2}$ are the obvious, most common compounds to consider, but other salts formed by chemical separation and refining should also be considered. These may be present in various states of chemical hydration.

Chemically bound water has an additional, second order effect on the SSF, due to secondary thermalization of the interrogating flux within the fissile material itself. There is a similar range of uranium compounds which should be considered.

It is planned to evaluate the effects of chemical form, density, enrichment and lump size based on a fixed interrogating flux spectrum. This will allow the worst case underestimation for each material type, to be assessed. This may be achieved using simple scaling rules rather than full $\mathrm{MCNP}^{\mathrm{TM}}$ modeling.

The primary shape - dependant parameter which is relevant in assessing the SSF, is the mean chord length, $r$, subtended by interrogating neutrons within the lump. For a fixed sample shape, SSF can be expressed, to first order, as a function of $r$. However, if one also considers lumps of different shapes, the distribution of path lengths within the sample, is also important as a second order effect. This means that although two different shaped lumps (same chemical form and enrichment) may have the same value of the mean chord length $r$, the SSF values will not be identical. It is possible to assess the shape - dependence of the self shielding factor, by plotting SSF against $r$, to obtain a different curve for different lump shapes. This will give an idea as to the magnitude of the systematic error associated with using a simple, single shape model.

Modeling different lump shapes could give useful information on the systematic uncertainties (see above) if it is assumed that the $U$ or Pu is always present as a single lump. A wide range of lump shapes is likely to occur in practice. For example, a pyramid of fixed aspect ratios could be used to represent accumulation in a fixed crevice in a particular plant item etc. If a particular waste stream consisted entirely of drums, each containing a single corner piece (the intersection of three sheets of metal) from the inside of a gloveboxes which tend to attract accumulation of fissile material dust or small particles, then one could imagine $U$ or Pu contamination building up in the corner, to give a pyramid. As more mass accumulates, the height of the pyramid changes but the aspect ratios remain roughly constant. So for this case an MCNPTM calibration of SSF versus pyramid height would make sense and represent what happens in practice. If a drum contains more than one corner piece (say 2), then we may get the same DDA response from either 2 equal pyramids or a single large one, where the effective SSF would of course be very different for these two situations. If one can assume that the mass is roughly equally distributed 


\begin{tabular}{|l|l|r|}
\hline & Canberra Industries, ACK\# D0043 & 33296 B \\
\hline Created: $29-$ September-2000 & IBIS Project & Revision B \\
\hline Revised: 13-January-2003 & Preliminary Design Study & Page 101 of 156 \\
\hline
\end{tabular}

between the two lumps, then the worst case underestimation would correspond to the SSF value for the smaller pyramid, rather than the single larger one. The ability to use spatial information to discount the single lump hypothesis is clearly beneficial here.

In most cases, lumps formed by accumulation of fissile material, could be present in an almost infinite range of shapes, from dilute material (tiny specks of dust adhered randomly to a surface), to say, wedge shaped pyramids. In between these two extremes there may be other shapes from accumulation in different shaped regions. For example accumulation in a join between two pipes or sheets of metal, as opposed to a corner piece, could give long slithers.

As an illustration of these shape - dependant effects, the results of a set of MCNPTM calculations is presented below. Calculations were performed for weapons grade Pu metal $(19.6 \mathrm{~g} / \mathrm{ml})$, in both sphere and infinite foil form. The interrogating flux spectrum was taken from a model of a typical californium shuffler. Although this spectrum is different to that for a DDA chamber, the results serve as a guide to magnitude of the effect of different shapes. For a given $r$, an infinite foil has a lower SSF value, due to the continuum of path lengths from zero to infinity (this contrasts with the case of a sphere in which case the maximum path length is equal to the sphere diameter).

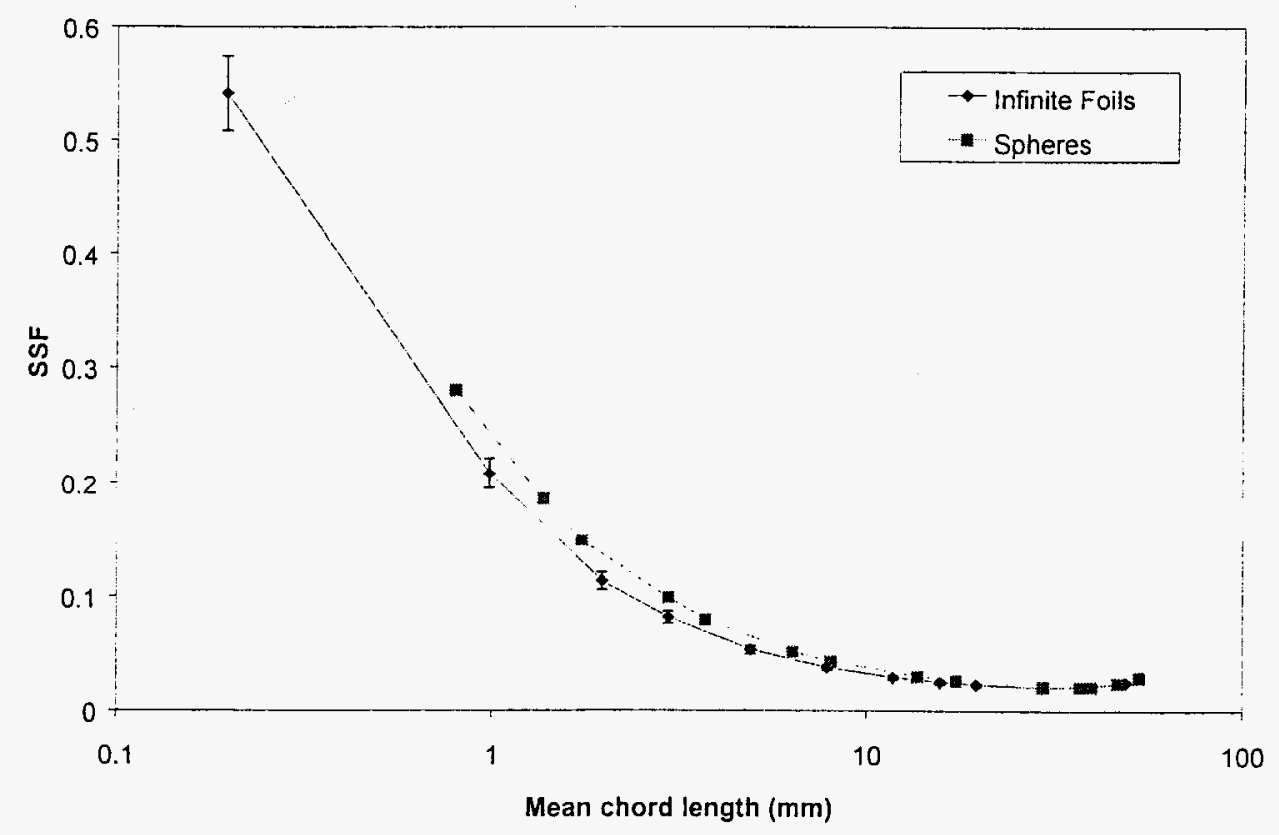

Figure 24 Comparison of SSF results for spheres and infinite foils of WG Pu metal 


\begin{tabular}{|l|l|r|}
\hline & Canberra Industries, ACK\# D0043 & 33296 B \\
\hline Created: 29-September-2000 & IBIS Project & Revision B \\
\hline Revised: 13-January-2003 & Preliminary Design Study & Page 102 of 156 \\
\hline
\end{tabular}

\section{Self shielding compensation techniques:}

The traditional approach to dealing with lumps when assaying with DDA instruments is to calibrate the system using realistic to pessimistic reference standards representative of the waste stream and then to expand the Total Measurement Uncertainty (TMU) band to encompass the worst case scenario. This remains a prudent approach. Furthermore it is usual to put reliance on the assay result only if it is below some fairly low safe working limit - typically of the order of 10 to 20 grams. With the IBIS system additional diagnostic information is available. For $\mathrm{Pu}-$ only wastes, the presence of lumps which are of consequence in the active mode assay have no significant impact on the passive neutron assay. This fact can be used in two ways. Firstly a discrepancy between the active and passive assay results can be used to flag the possible presence of lumps. Secondly, the ratio of the passive and active assay results can be used to derive an empirical active mode lump correction from in - service data which can then be applied in cases where the passive assay is unreliable (for example when $\mathrm{Cm}$ is present or when there is a high random neutron yield from the waste). In certain circumstances the differential attenuation between gamma - rays from the same nuclide can also be used to flag potential lumping. If detectable $(\alpha, \mathrm{x} \gamma)$ photons may also be indicative that $\mathrm{Pu}$ is present in compounded, rather than metallic, form. $\mathrm{PuF}_{4}$ is a favorable example. However, the principle benefit that IBIS brings to the treatment of lumps is as a result of its enhanced imaging capability over conventional systems. The gamma - ray data is acquired in scanning mode and this allows hot spots to be identified. The passive and active neutron data acquisition is also intended to allow spatial information to be extracted. Thus, if an assay result is sufficiently large that one would normally be concerned that there could be a significant under - reporting due to lumping, the system will clearly be able to indicate whether the material is present in the form of a single concentration or dispersed in several regions throughout the waste. If the latter is the case then clearly the worst case interpretation of a single spherical lump is inappropriate and can be relaxed to something more realistic. This extends the dynamic range of the active assay. This is particularly important in the case of $U$ wastes for which neither passive gamma nor passive neutron techniques offer any practically significant lump compensation facility. 


\begin{tabular}{|l|l|r|}
\hline & Canberra Industries, ACK\# D0043 & 33296 B \\
\hline Created: 29-September-2000 & IBIS Project & Revision B \\
\hline Revised: 13-January-2003 & Preliminary Design Study & Page 103 of 156 \\
\hline
\end{tabular}

\section{Integration of Neutron and Gamma-Ray Results}

The BSNS will provide separate quantitative and positioning information for spontaneous neutron sources and fissionable materials. The spatial resolution available is limited on physics grounds by the moderator and absorber content of the sample.

The BSGS can provide detailed isotopic data as a function of position within the waste crate for both $\mathrm{Pu} / \mathrm{U}$ isotopics and fission products and other species. The ability to measure fission products and other gamma-ray emitting isotopes reduces the reliance on process knowledge and scaling factors. Additionally, the isotopics data for a given sector of the waste crate can be combined with the BSNS assay result to correct the neutron assay for spatial variations of the isotopics distribution.

As part of the development effort, the gamma-ray transmission data may be integrated with the BSNS matrix mapping in the tomographic analysis. It is possible that the combined data can provide a more accurate assay result. These options will be evaluated in the work program. An indication of the potential of integrated data analysis in improving accuracy may be found in the discussion by Estep, et al. [Estep, et al, 1999].

\subsection{Introduction}

The IBIS generates a wealth of data which must be consolidated into a single, justifiable, best estimate assay result. This requires a software engine to be developed. Rather than begin this development from scratch it makes more sense to build the new engine around an established and proven product with inherent flexibility and solid version control. Canberra Industries standard software, NDA 2000, was considered as the effort to add comprehensive active data handling capability for a demonstration project was considered to be too time consuming. Consequently it is our intent to use an evolution of the LANL program CTEN_FIT.

In this section some of the features of the present version of CTEN_FIT will be outlined. This will serve to explain why we consider it to be an appropriate platform. The data integration issues will treated under CTEN_FIT philosophy. This provides a convenient interface and operational tool set.

The CTEN_FIT program, written for Windows $9 \times \mathrm{NTT}$ in $\mathrm{C}++$, performs databasing and analysis of combined thermal/epithermal neutron (CTEN) passive and active neutron assay data and integrates that with isotopics results and gamma-ray data from methods such as tomographic gamma scanning (TGS). The binary database is reflected in a companion Excel database that allows extensive customization via Visual Basic for Applications macros. Automated analysis options make the analysis of the data transparent to the assay system operator. Various record browsers and information displays simplify record keeping tasks. 


\begin{tabular}{|l|l|r|}
\hline & Canberra Industries, ACK\# D0043 & 33296 B \\
\hline Created: 29-September-2000 & IBIS Project & Revision B \\
\hline Revised: 13-January-2003 & Preliminary Design Study & Page 104 of 156 \\
\hline
\end{tabular}

The recently developed CTEN_FIT 1.0 software, [Estep, Buenafe, and Melton ,2000] which has been licensed to Canberra Industries[Canberra Industries] for distribution, was developed for analyzing passive and active neutron assays performed with the combined thermal/epithermal neutron (CTEN) [Melton, June 1997; Estep, et al., July 1998; Hollas, et al., 1997] prototype recently deployed at the Los Alamos RANT facility. A secondary goal was to simplify record keeping by databasing the data files, methods, and results of every analysis and placing copies of all database records in a Microsoft Excel spreadsheet for easy access by the user. The connection with Excel via OLE (object linking and embedding) automation has the added advantage of allowing extensive customization of analysis methods and reporting through Visual Basic for Applications (VBA) macros, which are Visual Basic programs that have direct access to the contents of the Excel speadsheet. Using VBA macros, the user can: extract selective information from the database records; perform calculations; create dialogs; add additional information to each record as it is created; call user-written $\mathrm{C}++$ or Fortran modules; and, in general, add any kind of analysis algorithms or output forms desired.

The CTEN method, like other passive active neutron (PAN) assay methods, combines two analysis techniques: passive neutron coincidence counting (PNCC) and the active differential dieaway technique (DDT). Several options are available for analyzing this data. Depending on the analysis path, the two methods may be used separately or in combination. Some CTEN analysis options, for example, use active data to derive matrix corrections for use in the passive analysis. It was desired that CTEN_FIT offer the option to integrate the analysis of PNCC and DDT assays with isotopic composition information generated by programs such as MGA, [Young, Ruhter, and Gunink] so that output forms summarizing analysis results could report masses for the isotopes required by WIPP. With all this complexity already required the development of CTEN_FIT was taken a step further by incorporating support for the databasing and analysis of data from gamma-ray methods such as TGS (tomographic gamma scanning). Thus, CTEN_FIT provides for the simultaneous analysis and databasing of four different techniques at once: DDT, PNCC, isotopics, and TGS.

Specific data file types and formats are required for analysis of the CTEN_FIT DDT and PNCC method data, to support the significant "built-in" analysis capabilities for those methods. However, for the isotopics and TGS analyses no part of the analysis is built in, not even file reading. All that is provided is a base level of databasing (file names, etc.), the ability to specify analysis options in method records, and the ability to register VBA macros to be called for file reading, analysis, and output. The TGS support also allows interfacing to the Los Alamos TGS_FIT software, which is used for image reconstruction and analysis of TGS, DDT, and PNCC data. Because of this flexibility, referring to the fourth analysis method as TGS is merely a suggestion. Any kind of analysis of any kind of data can be incorporated via this fourth data type. As an example, we have plans to database and integrate non-TGS transmission-corrected gamma-ray assays of crate counters in the IBIS [Croft, et al., May 2000] system using the "TGS" support in CTEN_FIT. 


\begin{tabular}{|l|l|r|}
\hline & Canberra Industries, ACK\# D0043 & 33296 B \\
\hline Created: 29-September-2000 & IBIS Project & Revision B \\
\hline Revised: 13-January-2003 & Preliminary Design Study & Page 105 of 156 \\
\hline
\end{tabular}

Mastery of the CTEN_FIT program requires some effort, because of the databasing and the extensive setup options. However, once set up its use can be made completely transparent to the NDA operator through automatic analysis options. The recent application to the CWAM [Melton, Estep, and Peterson, May 200] (crated waste assay monitor) instrument delivered to Oak Ridge/Y12 is a case in point. In the base configuration, the CWAM operator performs an active DDT assay (no passive assay is done, as the assay is for uranium) using the Los Alamos WIN_CTEN [Estep, et al., April 2000] data acquisition software. When the assay is finished, WIN_CTEN transmits the "c10_analyze_all" message to the CTEN_FIT program via the Windows message queue (i.e., using the Windows API PostMessage() function). This initiates the CTEN_FIT automatic database update and analysis sequence, which reads data files from a specified active data directory into the database and then analyzes any unanalyzed data records using a method specified for automatic analysis (up to eight different methods can be specified for automatic analyses, but CWAM currently uses only one). A custom VBA print macro is then called to print out results of the assay. All this happens in the background with no intervention required on the part of the operator.

Automatic analysis is provided to reduce the skill and training requirements for new operators, but a direct, foreground use of the CTEN_FIT software offers many powerful features that are worthwhile to learn. These include an electronic logbook that automatically records all events and user actions as well as database access features that allow browsing and manipulation of previous assays and analyses. Full documentation of these features can be found in the "CTEN_FIT User's Manual" [Estep, et al., April 2000] and related documentation. In this section we summarize some of the main features available.

\subsection{Specifications}

The CTEN_FIT version 1.0 program was written in $\mathrm{C}++$ using the Microsoft Visual $\mathrm{C}++6.0$ compiler. It runs under Windows 95 and 98 . Although it has never been tested with any versions of Windows NT, there is no reason to think it would not run under NT operating systems, as no special hardware interfacing is involved. CTEN_FIT has been run on 133-MHz and faster PC clone computers and with no performance problems. The slowest parts of the program are those that involve transfer of data between CTEN_FIT and Microsoft Excel via OLE, which involve noticeable time delays. We are examining methods to speed that transfer in future revisions.

The currently distributed version (1.0) of CTEN_FIT runs only with Microsoft Office 97' installed, and not with the recent Microsoft Office 2000.

\subsection{The CTEN_FIT DATABASE}

There are six primary record types in CTEN_FIT and several supporting record types. The four primary data record types have subtypes for normal, calibration, and background data and analyses. All the CTEN_FIT record types are listed in Table 13, below. 


\begin{tabular}{|l|l|r|}
\hline & Canberra Industries, ACK\# D0043 & 33296 B \\
\hline Created: 29-September-2000 & IBIS Project & Revision B \\
\hline Revised: 13-January-2003 & Preliminary Design Study & Page 106 of 156 \\
\hline
\end{tabular}

Table 13. CTEN_FIT Database Record Types and Subtypes

\begin{tabular}{|c|c|c|c|}
\hline Type & Subtype & Excel Worksheet & Description \\
\hline Active & normal assay & Active Data & \multirow{3}{*}{$\begin{array}{l}\text { Active (DDT) data files are recorded, } \\
\text { along with some pre-analysis of scaler } \\
\text { mode data. }\end{array}$} \\
\hline Active & calibration & Active Calibration & \\
\hline Active & background & Active Background & \\
\hline Passive & normal assay & Passive Data & \multirow{3}{*}{$\begin{array}{l}\text { Passive (PNCC) data files are recorded } \\
\text { No analysis is done. }\end{array}$} \\
\hline Passive & calibration & Passive Calibration & \\
\hline Passive & background & Passive Background & \\
\hline Isotopics & all & Isotopics Data & Isotopics results files are recorded. \\
\hline TGS & normal assay & TGS Data & \multirow{3}{*}{$\begin{array}{l}\text { TGS or other method file names are } \\
\text { recorded. }\end{array}$} \\
\hline TGS & calibration & TGS Calibration & \\
\hline TGS & background & TGS Background & \\
\hline Results & normal assay & Results & \multirow{3}{*}{$\begin{array}{l}\text { Results from an analysis are recorded, } \\
\text { with references to all data records and } \\
\text { the single method record employed. }\end{array}$} \\
\hline Results & calibration & Calibrations & \\
\hline Results & background & Background & \\
\hline Batch & - & Batch & For recording analysis batches. \\
\hline Method & - & Method & Description of an analysis method. \\
\hline \multicolumn{4}{|c|}{ The next two record types are referenced by method records. } \\
\hline $\begin{array}{l}\text { Neural } \\
\text { Network }\end{array}$ & - & Neural Network & $\begin{array}{l}\text { Description of a neural network or other } \\
\text { external executable to call. }\end{array}$ \\
\hline $\begin{array}{l}\text { Tomograph } \\
\text { y }\end{array}$ & - & Tomography & $\begin{array}{l}\text { Setup information for tomographic } \\
\text { imaging }\end{array}$ \\
\hline \multicolumn{4}{|c|}{ The remaining entries are not records, but spreadsheets that can be referenced } \\
\hline- & - & Constants & User's constants \\
\hline- & - & Setup & Copy of database setup file \\
\hline- & - & Scratch Pad & Used to communicate with VBA macros \\
\hline
\end{tabular}

The CTEN_FIT database is actually a binary file with default extension .CDB, which is mirrored (almost) in an Excel XLS file with the same title. Both are necessary. The Excel database file contains all user macros and, possibly, user-defined worksheets to support customized analysis, while the binary database contains indexing and security information not found in the Excel file. The Excel database contains a worksheet for each record type, with the names listed in Table 13 The first row in each worksheet lists names for the various information fields in each record. Starting in row two, each worksheet row contains a different record. Figure 25 illustrates a segment of the Excel database file for the Active Data worksheet, which stores Active Data records. The quantities listed in each column are scaler sums that are defined and named in the database setup file. These are used only in the CTEN system installed at RANT. The CWAM system uses a different set of scaler sums in its analysis. 


\begin{tabular}{|l|l|r|}
\hline & Canberra Industries, ACK\# D0043 & 33296 B \\
\hline Created: 29-September-2000 & IBIS Project & Revision B \\
\hline Revised: 13-January-2003 & Preliminary Design Study & Page 107 of 156 \\
\hline
\end{tabular}

\begin{tabular}{|c|c|c|c|c|c|c|c|c|c|}
\hline & AN & $1 \mathrm{AB}$ & 3 & 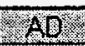 & & & & 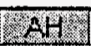 & \\
\hline & efmTotal & +1. & SfmSum & +1 & Bfm4 & +1 & Dfm4 & $+1-$ & Efm \\
\hline $4 y^{2} \times 1$ & 23412 & 153.01 & 33587 & 183.3 & 117270 & 342 & 30728 & 175.3 & 2779 \\
\hline & 23507 & 153.32 & 34156 & 184.8 & 117871 & 343 & 31176 & 176.6 & 065 \\
\hline & 23349 & 152.8 & 33819 & 183.9 & 117 & 342 & 31 & 176.4 & 495 \\
\hline & 24669 & 157. & 99 & 188.7 & 7 & 35 & 32539 & 180.4 & 649 \\
\hline & 23615 & 153 & 4 & 186.7 & 12 & 3 & 21 & 178.7 & 533 \\
\hline & 24864 & 157.68 & 36273 & 190.5 & 125365 & 354 & 33258 & 182.4 & 17.10 \\
\hline & 23822 & 154.34 & 35091 & 187.3 & 120 & 348 & 31690 & 178 & 17.185 \\
\hline & 22543 & 150.14 & 9537 & 97.66 & 187312 & 433 & 36712 & 191.6 & 20.875 \\
\hline & 22433 & 149.78 & 9292 & 96.4 & 187624 & 433 & 36780 & 191.8 & 0091 \\
\hline & 22910 & 151 & 9641 & 98.19 & 189388 & 435 & 36973 & 192.3 & 173 \\
\hline & & & & & & -5 & $=115$ & & 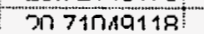 \\
\hline
\end{tabular}

Figure 25. A section of the Active Data worksheet from the Excel database portion of a sample database. Each row after the first corresponds to a separate Active Data record. The quantities shown are various scalers sums, which are named and defined in the database setup file.

The user can create as many CTEN_FIT databases as desired, although only one database can be accessed at a time. It is a matter of taste whether to put a year's worth of data into every database or to start a new database every week.

The databasing in CTEN_FIT is intended to be used for the automation of quality assurance tasks that are now done by hand and to provide complete traceability on all analyses. This is accomplished through various design and security features, which are too numerous to describe in detail here. We will mention just a few security features that make CTEN_FIT a useful quality assurance tool. Every record of any type is dated and given a unique serial number and can never be deleted from the database once entered. Method records, for example, can be freely edited without changing the method name, and will appear to the user to have been changed for subsequent analyses. However, the original record remains in the database and will still be associated with any analyses that referenced that particular version of the method. The creation date, last modification date, and size of external files used in analyses are monitored to ensure than no alteration or substitution of those files has occurred. There are (optional) separate passwords associated with the binary and Excel database files, preventing them from being opened by an unauthorized party either in CTEN_FIT or Excel. This feature also protects Excel user macros from being altered without authorization. The individual cells of the Excel database file can optionally be locked to prevent modification by any user (the randomly generated password, embedded in the binary CDB file, would be known only to the CTEN_FIT program). The automated electronic logbook, though not password protected itself, can be protected by user procedures and records all transactions that take place in the database.

All CTEN_FIT records contain generic information such as a unique record serial number, the time and date the record was created and who created it, and other bookkeeping information. Beyond that, the information stored varies by record type. The different record types are described below. 


\begin{tabular}{|l|l|r|}
\hline & Canberra Industries, ACK\# D0043 & 33296 B \\
\hline Created: 29-September-2000 & IBIS Project & Revision B \\
\hline Revised: 13-January-2003 & Preliminary Design Study & Page 108 of 156 \\
\hline
\end{tabular}

\subsubsection{Data records}

The data records (Active, Passive, Isotopics, and TGS) contain the file name and path of the data file(s) comprising a single analysis of that type, the name of the operator who collected the data and any comments, the sample ID, and similar information. The Active records use only WIN_CTEN-generated ADA, AQ1, and AQ2 data files (scaler mode data and shielded and bare detector list-mode files, respectively), and require those file extensions and formats. Assay data sufficient for most non-imaging, non-list-mode DDT analyses is extracted from the ADA scalermode files and stored in the Active record. The Passive records use only WIN_CTEN-generated PDA, PQ1, and PQ2 data files (scaler mode data and shielded and bare detector list-mode files, respectively). Bookkeeping information (operator name, comment, etc.) is extracted from the PDA file and stored in the Passive record, but no real data is stored. Bookkeeping information and data is read into Isotopics and $T G S$ records by user-written, custom VBA macros that are registered in the database setup file used to generate the database. The file types supported are also specified in the setup file by their file extensions. We currently use a macro named $R E A D_{-} T M A$ and the TMA extension for preanalyzed TGS results files in our test/example cases.

\subsubsection{Method and Related Records}

Each Method record defines a way of analyzing the data by specifying the data types required for the analysis and the specific analysis methods to be used. Generally, CTEN_FIT only does part of a passive or active neutron analysis, leaving the remaining analysis and reporting of results for two Neural Network executables, three sets of Tomography matrix image reconstructions, and three user-defined VBA macros to carry out. The way this is done is illustrated in Figure 20, below, which shows the Edit Method Record dialog used for creating and editing Method records. This dialog is accessed by clicking the Setup/Method Setup menu option, followed by either the Edit Method or Add New Method buttons. The particular method shown, named "PAN with isotopics," uses the external executable defined in a Neural Network record named "PAN Matrix CF" to create a matrix correction factor for the active analysis (although it is not obvious, a matrix correction is also returned for the passive analysis). The built-in passive list mode analysis generates various histograms, moments, and coincidence rates used in the Gray Barrel analysis methods of Brunson and Nicholas [1992] The remaining active and passive analysis is done by VBA macro I, named $P A N \_M A C R O$, and by macro II, named PassOnly. The $P A N \_M A C R O$ macro calls two other macros, $\bar{A} c t M a c$ and PassMac2, in succession, which is the method used to expand the three-macro-per-method allotment to an arbitrary number of macros. Preanalyzed isotopics results are integrated into the output in the print macro named PAN_ISO_Print, which reports one active and two passive results (from two different methods). A recommendation of which result to use is made, based on various indicators. The check box in the lower right flags this method for use in automatic analyses. 


\begin{tabular}{|l|l|r|}
\hline & Canberra Industries, ACK\# D0043 & 33296 B \\
\hline Created: 29-September-2000 & IBIS Project & Revision B \\
\hline Revised: 13-January-2003 & Preliminary Design Study & Page 109 of 156 \\
\hline
\end{tabular}

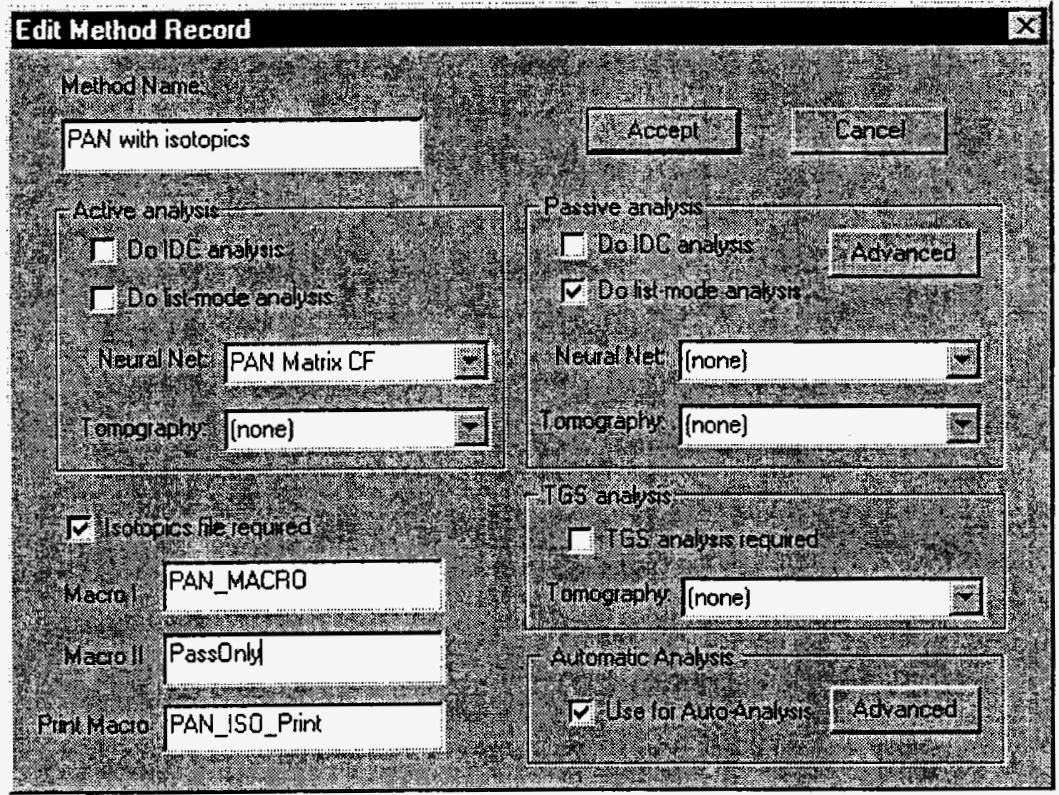

Figure 26. The Edit Method Record dialog, used for creating or editing method records. This dialog is accessed by clicking the Setup/Method Setup menu option, followed by the Add New Method or Edit Method buttons

At first glance the dialog in Figure 26 may seem complex, but most of the detail is encapsulated by simply named references to supporting records and macros, or by supporting dialogs. The automatic analysis and passive list options are set in the "Advanced" dialogs for those two categories. The Neural Net and Tomography selections correspond to records of those types, with their detailed settings encapsulated in a single name that displays in drop-down lists. The VBA macros can conceal an unlimited level of detail. Considering that it totally specifies an integrated analysis of up to four measurement techniques at once, or any combination thereof, it's actually a fairly simple dialog to use.

Figure 27 shows the Add Neural Network/Exe Record dialog box, accessed by clicking the Setup/Neural Network Setup menu option and then the Add Record button. This dialog is being used to create the Neural Network record named "PAN Matrix CF." The record specifies that at a well defined point in the analysis sequence an external executable file named NN.EXE

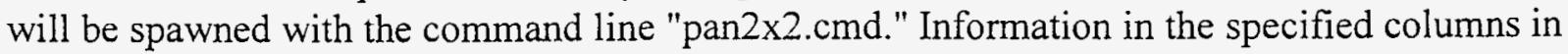
the "input" Active Data worksheet is placed in a ASCII file named "temp.in" to be used as input by the executable (NN.EXE). The executable is expected to create an ASCII file with the name "temp.out," which will be read by CTEN_FIT to extract four floating point numbers that will be stored in the specified columns in the "output" Results worksheet. The rows to be accessed in the input and output worksheets correspond to those of the Active and Results records involved in the of analysis. (The rule is that for the input worksheet, if a data-type worksheet is referenced and is involved in the analysis, the row number is determined by that record's index. In all other cases the Results index will be used to compute the row number.) Data and Results worksheets 


\begin{tabular}{|l|l|r|}
\hline & Canberra Industries, ACK\# D0043 & 33296 B \\
\hline Created: 29-September-2000 & IBIS Project & Revision B \\
\hline Revised: 13-January-2003 & Preliminary Design Study & Page 110 of 156 \\
\hline
\end{tabular}

that have subtypes will be permuted to the correct subtype. That is, if the analysis is performed on background data, the Active Background and (results) Background worksheets will be accessed instead of the specified Active Data and Results worksheets, which are for normal assays.

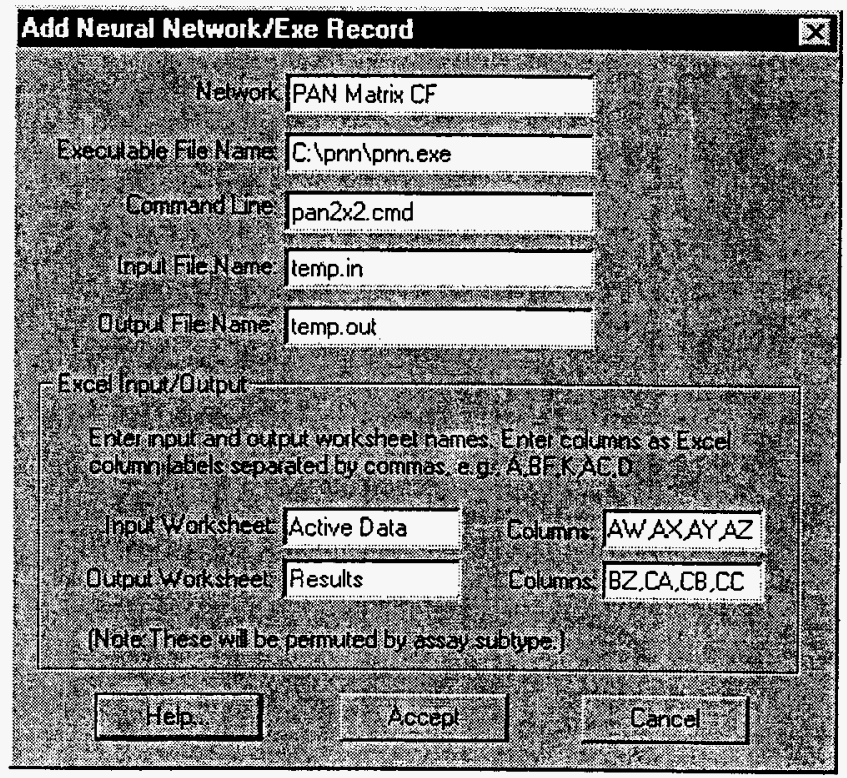

Figure 27. The Add Neural Network/Exe Record dialog used to create Neural Network records. This dialog is accessed by clicking the Setup/Neural Network Setup menu option, followed by the Add Record button.

In the Neural Network record being created in Figure 27, the NN.EXE program called is a probabilistic neural network program originally called PNN that was modified to allow a command file name to be passed in the command line ("pan $2 \times 2 . c m d "$ in the example). However, the executable does not have to be a neural network program, but can be any kind of program. Note that whenever a Neural Network record is created a test is done to verify the existence of the executable. Its creation date, last modification date, and size are recorded in the Neural Network record. If any of these change, an error will be generated whenever that Neural Network record is accessed in an analysis. This protects against alteration or substitution of the executable, which in effect would be an alteration of the method itself and would defeat the security measures that safeguard the integrity and traceability of the Method and Results records.

The Tomography record stores setup information, such as scanning resolutions and other dimensions, along with the names of the binary tomographic matrix files (EMT, AMT, and TMT files) that are used in the TGS_FIT [Estep, to be published; Estep and Cavender, 1994] program to define the geometry of the problem. A command file name can also be specified that will perform the TGS_FIT analysis. As with Neural Network records, all files referenced by the Tomography record are safeguarded by verification of their creation and last modification dates and file size 


\begin{tabular}{|l|l|r|}
\hline & Canberra Industries, ACK\# D0043 & 33296 B \\
\hline Created: 29-September-2000 & IBIS Project & Revision B \\
\hline Revised: 13-January-2003 & Preliminary Design Study & Page 111 of 156 \\
\hline
\end{tabular}

\subsubsection{Results Records and the Analysis Queue}

A Results record, which is also be called an "analysis set," records the details of every analysis, referencing back to the data records used in the analysis as well as to the Method record that describes how the analysis will be or was done. The ambiguity over whether the analysis was done or not exists because one initially creates "partial" Results records that include all the information needed for the analysis, but that have not been analyzed yet. These unanalyzed records are said to be in the "analysis queue" awaiting submission for analysis. One can create as many partial results records as desired before submitting them for analysis. Schematically, this can be represented as

(As required: Active + Passive + Isotopics + TGS)

+ Method $\rightarrow$ partial Results (added to queue)

followed by processing of the analysis queue (for all partial Results records),

$$
\text { partial Results } \rightarrow \quad \text { Analysis } \rightarrow \text { completed Results records. }
$$

Results records can be created and processed automatically by pressing the Run Analyses button on the main window, which both creates the analysis queue and then submits it for analysis, or equivalently by clicking the Analysis/Update Analysis List and Analysis/Run Analysis List menu options in succession. These, as implied, create the analysis queue and submit it for analysis, respectively. The automatic analysis loops through all the Methods flagged as automatic and searches the database by subtype for data records that are required by the method and that have not yet been analyzed by that method. Following a set of simple rules based on the declared sample ID, data records of the different required types are associated into a partial Results record. Only data records with the same sample ID will be associated into a Results record and no data record will be used twice for the same method. Therefore, there can be left-over data records that could not be matched and would presumably be picked up in later automatic analyses.

Much greater flexibility is allowed in manually associating data files with a method to create a partial Results record. Figure 28 shows the Create Analysis Sets dialog used for this purpose, which is accessed by clicking the Analysis/Create Analysis Sets menu option. The "Pan with isotopics" method is selected as the Method record to be used, and data records of subtype "Normal" are listed by type in four list boxes. The data types that are required in this method (Active Data, Passive Data, and Isotopics, but not TGS Data) are indicated by check marks. In this example case the user has followed the recommended file naming convention of naming data files by their sample ID plus a ordinal number used to distinguish repeat assays. The WIN_CTEN software offers this convention as one method of automatic file naming (the others conventions offered are based on the date or a consecutive run number). The sample ID that appears on the first line in the small information box below each list box came from the data file itself, except in the case of the isotopics (.TXT) file, which does not store that information. The READ_FRAM 


\begin{tabular}{|l|l|r|}
\hline & Canberra Industries, ACK\# D0043 & 33296 B \\
\hline Created: 29-September-2000 & IBIS Project & Revision B \\
\hline Revised: 13-January-2003 & Preliminary Design Study & Page 112 of 156 \\
\hline
\end{tabular}

macro (macro II) registered for this database (independent of the method) uses the file name itself to deduce the sample ID and enter it in the database. To create a results record, one selects one of each required data type and presses the Add to Queue button, which creates a partial results record and displays it as a single line-entry in the information box at the bottom of the dialog.

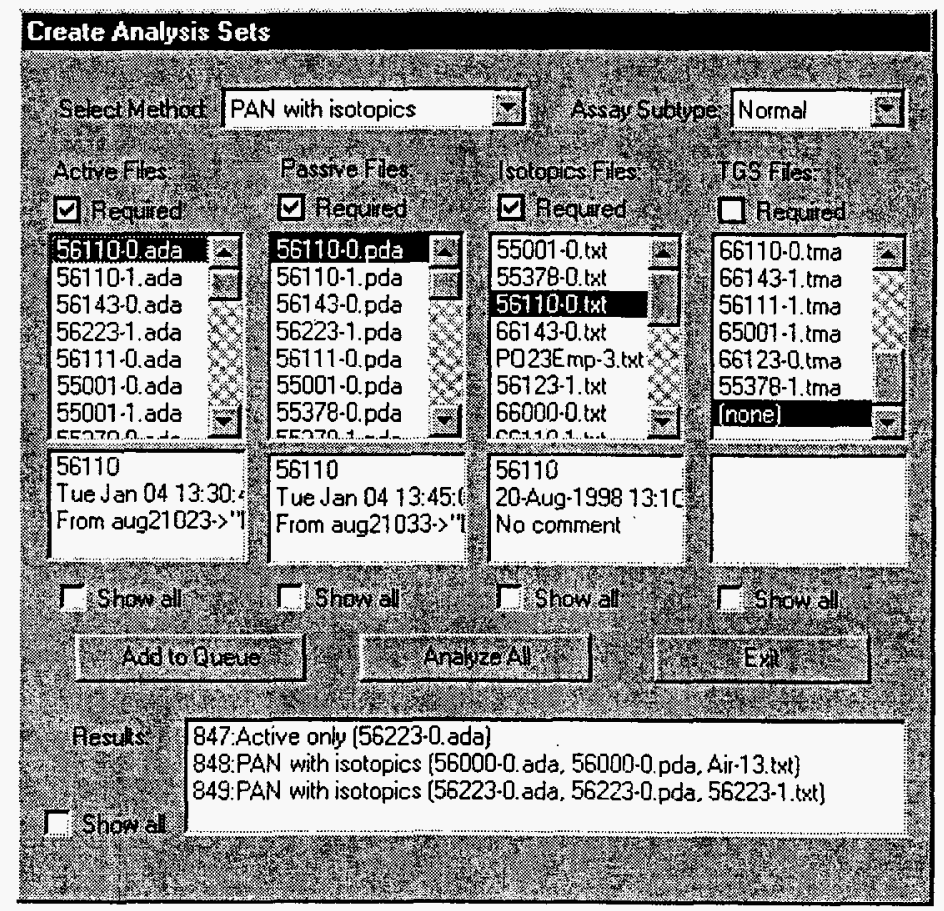

Figure 28. The Create Analysis Sets dialog, used to associate data records with a Method record to create a Results record in the analysis queue. This dialog is accessed by clicking the Analysis/Create Analysis Sets menu option.

In the example in Figure 28, three previously created partial results records are shown (the entries show the results record serial number, the method name, then the file names for the data records used in parentheses). These three partial results records constitute the normal portion of the current analysis queue (there could be additional, entries not shown for the calibration or background subtypes). Notice the results record number 848, for which (presumably) no isotopics assay was done on the drum and the substitute assay "Air-13.txt" was used instead. This sort of substitution can only be done using the Create Analysis Sets dialog. Three data files with sample ID "56110" are currently selected and would be appended to the analysis queue if the Add to Queue button were clicked.

\subsection{The CTEN_FIT Main Window}

Figure 29 shows the CTEN_FIT main window, with the Active Data record browser selected. The different browsers and information windows are selected by a combination of tab controls (along the top of the information window) and by the five buttons that run from left to right along the bottom, each of which brings up a different tab set. The Data, Background, and Calibration 


\begin{tabular}{|l|l|r|}
\hline & Canberra Industries, ACK\# D0043 & 33296 B \\
\hline Created: 29-September-2000 & IBIS Project & Revision B \\
\hline Revised: 13-January-2003 & Preliminary Design Study & Page 113 of 156 \\
\hline
\end{tabular}

button switch between the browser displays for those subtypes, all of which have the same tab selections. The Images button brings up various tomographic image displays, the image displays are not implemented in the CTEN_FIT version currently in use in the field, while the History button brings up a scrolling edit box that shows the most recent automatic electronic logbook entries.

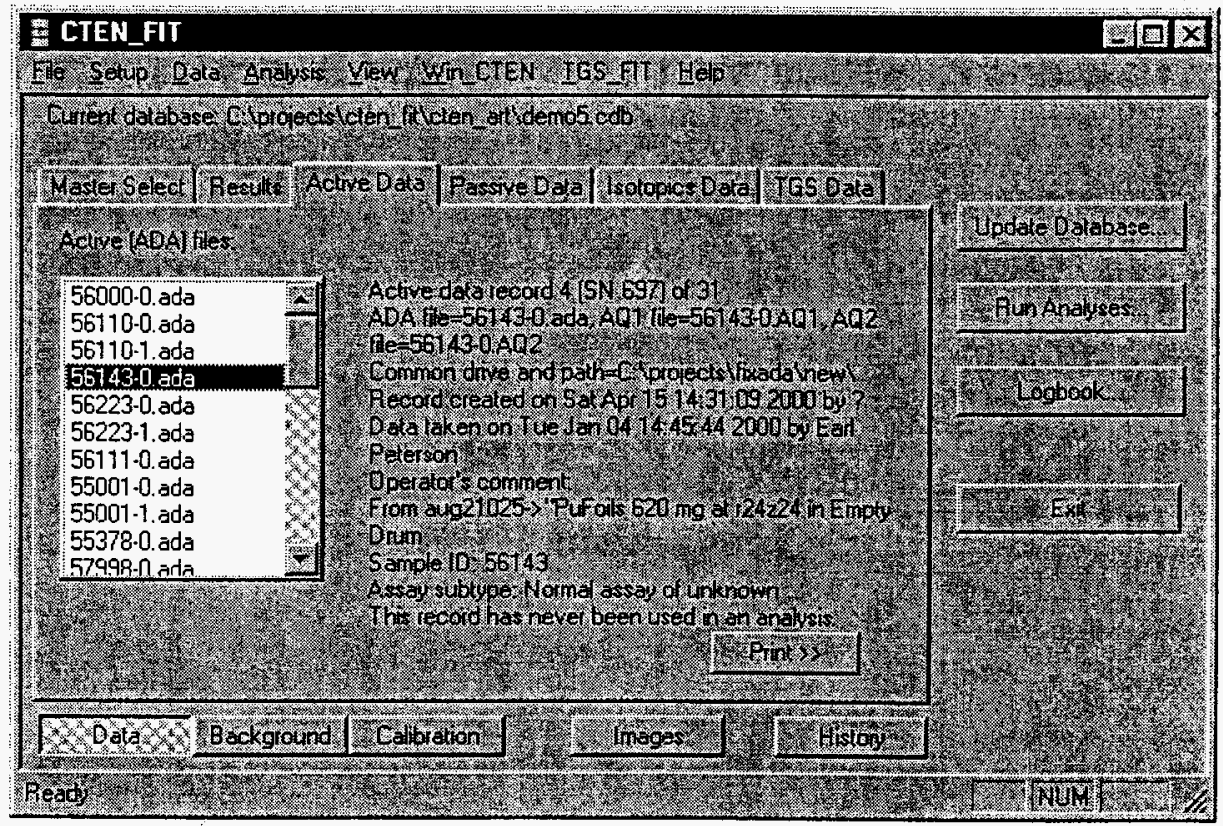

Figure 29. The CTEN_FIT main window, with the active (normal data) browser selected. Clicking the Print button will print detailed information about the selected record to the default printer.

All necessary database functions (which excludes the browsers and other information displays) can be executed using the pull-down menu items. However, to simplify the most common operations, three useful buttons are located along the right side of the main window. The Update Database button reads new data files into the database, while the Run Analyses button commences automatic analysis. The Logbook button brings up a copy of the electronic logbook in an easy-to-use ASCII text editor (PFE32 [Phillips) that allows viewing, editing, and printing of the logbook. An excerpt from a typical logbook is shown below (some text to the right has been truncated).

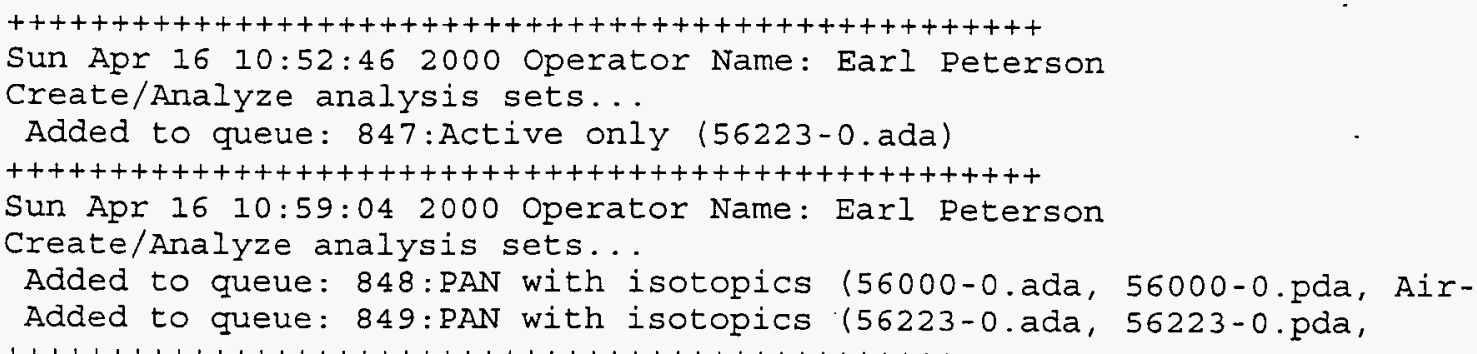




\begin{tabular}{|l|l|r|}
\hline & Canberra Industries, ACK\# D0043 & 33296 B \\
\hline Created: 29-September-2000 & IBIS Project & Revision B \\
\hline Revised: 13-January-2003 & Preliminary Design Study & Page 114 of 156 \\
\hline
\end{tabular}

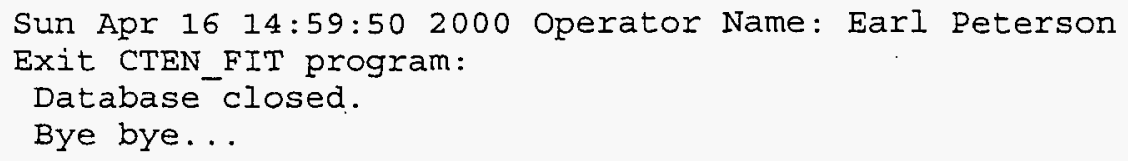

\subsection{Nomenclature}

The following conventions are used in referencing various components of the CTEN_FIT software.

- Record types are indicated in bold italics (e.g., Active Background) and will have the same name as the label on their corresponding Excel worksheet. Data types with unspecified subtypes will be referenced the same way (e.g., Active), although there is no corresponding Excel worksheet in that case.

- Record subtypes (normal, background, and calibration) are written in ordinary type.

- Menu items are referenced in bold type, with a slash separating different levels of the menu (e.g., Analysis/Create Analysis Sets). Underscores and ellipses are suppressed. Dialog buttons or other controls are also referenced in bold type.

- Dialog names are referenced in underlined bold text (e.g., Create Analysis Sets)

- VBA macros are referenced in italics (e.g., READ_TMA). 


\begin{tabular}{|l|l|r|}
\hline & Canberra Industries, ACK\# D0043 & 33296 B \\
\hline Created: 29-September-2000 & IBIS Project & Revision B \\
\hline Revised: 13-January-2003 & Preliminary Design Study & Page 115 of 156 \\
\hline
\end{tabular}

\section{Conclusions}

The CTEN/CWAM technology in the BSNS offers the ability to obtain both sensitive and accurate assay for heterogeneous source and matrix distributions. The sensitivity for plutonium and uranium assay is obtained using combined passive and active neutron interrogation based on the Differential Die-Away technique. Tomographic analysis techniques are used to correct for source positioning and matrix heterogeneity. Scanning AAS is used to derive matrix heterogeneity profiles and correction factors. The BSGS offers position-dependent information on gamma emissions and isotopics as well as on density distributions.

This set of position-dependent information will be the most complete ever assembled for crate assay, giving the maximum opportunity to characterize the complex distribution of matrix and isotopes over the volume of the crate, and so make the assay more accurate. The program will identify the main parameters useful as characteristics in the analysis of the waste form and evaluate their effectiveness and optimum combination in deriving the best measurement performance. The IBIS performance will then be evaluated against all the limits of detection and accuracy requirements in the original specification. The combination of techniques and completeness of the information promise to provide the most accurate assay of crated wastes of interest to the DOE community. 


\begin{tabular}{|l|l|r|}
\hline & Canberra Industries, ACK\# D0043 & 33296 B \\
\hline Created: 29-September-2000 & IBIS Project & Revision B \\
\hline Revised: 13-January-2003 & Preliminary Design Study & Page 116 of 156 \\
\hline
\end{tabular}

\section{Appendices}

\section{A. First Principles Derivation of the FNDP Intrinsic Decay Time Constant}

B. Measured Response of the CWAM System

C. New Work

D. References and Bibliography 


\begin{tabular}{|l|l|r|}
\hline & Canberra Industries, ACK\# D0043 & 33296 B \\
\hline Created: 29-September-2000 & IBIS Project & Revision B \\
\hline Revised: 13-January-2003 & Preliminary Design Study & Page 117 of 156 \\
\hline
\end{tabular}

\section{A. First Principles Derivation of the FNDP Intrinsic Decay Time Constant}

The fast neutron detector packages consist of ${ }^{3} \mathrm{He}$ filled proportional counters, sensitive primarily to thermal neutrons, embedded in a polyethylene moderator covered by cadmium which is opaque to thermal neutrons. Fast neutrons entering the package are rapidly slowed down to thermal energies in the moderator on a time scale of a few $\mu$ [Glasstone and Edlund, 1957]. The thermalized population then decays approximately exponentially with a characteristic die-away time which is determined by the absorption and diffusion loss mechanisms at work. These are set by the materials and geometry of the package. Neutrons can be lost by parasitic capture in the polyethylene and stainless steel walls of the proportional counters or by leaking from the system which essentially means capture in the Cd layers. Neutrons interacting with the ${ }^{3} \mathrm{He}$ are removed from the population but are registered as counts. For gas like moderators (so that Bragg scattering and solid state effects due to atomic binding can be neglected) and pure $1 / \mathrm{v}$ absorbers the decay of the thermal neutron population in a finite medium may be described as the sum of decaying exponential modes and a continuum representing the temporal collapse of the various spatial modes. The process is balanced such that the spectrum remains the same shape; that of a Maxwellian in thermal equilibrium with its surroundings. Perturbation methods can be applied to the transport equations to develop the dependence of the decay modes on the size of the system. In general the longest lived, fundamental mode, decay constant is a principle concern in governing the practical aspects of the measurement system.

The importance of the intrinsic decay time constant is twofold. Firstly, in passive neutron coincidence counting it is one of the temporal factors that ensures that spontaneous fission neutrons which have a common origin (in both time and space) do not reach the counters simultaneously causing paralyzation, rather the burst is dispersed in time over a period characteristic of the die-away time of the system. The neutrons remain correlated in time over intervals commensurate with the mean lifetime of a neutron in the assembly.

Time correlation analysis of the ensuing pulse train enables the multiplicity of the individual bursts to be unraveled. A short die-away time for the system as a whole (consistent with the amplifiers being able to recover before the next pulse is detected and acceptable dead-time losses) is desirable in order to minimize the likelihood of Accidental coincidences between noncorrelated events. The statistical precision of the genuine correlated signal is poorer as the Accidentals rate increases. This is a concern when the $(\alpha, n)$ rate in the waste exceeds the (SF, $n$ ) rate by factors greater than a few. 


\begin{tabular}{|l|l|r|}
\hline & Canberra Industries, ACK\# D0043 & 33296 B \\
\hline Created: $29-$ September-2000 & IBIS Project & Revision B \\
\hline Revised: 13-January-2003 & Preliminary Design Study & Page 118 of 156 \\
\hline
\end{tabular}

The second reason for preferring a short die-away time is the relation to level of active background that is present following the firing of the $14 \mathrm{MeV}$ neutron generator. This sets the minimum period after the shot that one would chose to open any signal counting gate. Consider for example, a neutron generator pulsing at a rate of $\mathrm{p}(\mathrm{Hz})$ and producing $\mathrm{Y}$ neutrons per pulse. For an assay period of $t(\mathrm{sec})$, the number of direct counts, $C_{D}$, appearing in the detector gate of duration $\operatorname{Tg}(\mathrm{sec})$ opened a recovery period $\mathrm{Tp}(\mathrm{sec})$ after the pulse, which we will take to be short in comparison to either $\tau$ or $\mathrm{Tg}$, will be given by:

$$
\begin{aligned}
C_{D}=\varepsilon_{D} \cdot p \cdot Y \cdot t \cdot \int_{x=T_{p}}^{x=\left(T_{p}+T_{g}\right)} e^{-x / \tau} \cdot \frac{d x}{\tau} \\
=\varepsilon_{D} \cdot p \cdot Y \cdot t \cdot e^{-T_{p} / \tau} \cdot\left(1-e^{-T_{g} / \tau}\right)
\end{aligned}
$$

where $\varepsilon \mathrm{D}$ is the efficiency of detection for neutrons direct from the neutron generator. In order to maintain a high signal-to-background ratio one would clearly like to keep $\varepsilon D$ low (most likely by geometrically separating the generator and the FNDP's and using the waste filled container as a shield), and operate with a high $(\mathrm{Tp} / \tau)$ ratio. There is a pressure to keep Tp as low as practical in order to make best use of the flux and so this translates into a pressure to keep $\tau$ low.

The intrinsic die-away time, $\tau$, is a property solely of the FNDP geometry and its contents. From one-group diffusion theory, which is equivalent to a P1 approximation [Bell and Glasstone, 1970] an estimate of $\tau$ can be made using Equation 22 [Glasstone and Edlund, 1957].

$$
1 / \tau=v \cdot \Sigma_{a}+D \cdot v \cdot B^{2}-C \cdot B^{4}+\ldots, \sec ^{-1}
$$

where, in the case of $1 / \mathrm{v} "$ absorbers and a thermal Maxwellian energy spectrum:

$v=\quad$ the neutron speed at which parameters are evaluated; usually this is $2.2 \times 10^{5} \mathrm{~cm} . \mathrm{s}-1$ for a Maxwellian thermal flux

$D=$ the average diffusion coefficient of the moderating medium within the package in units of $\mathrm{cm}$.

$B^{2}=$ is the geometrical buckling of the package in units of $\mathrm{cm}^{-2}$.

$C=$ the diffusion cooling term and is usually entirely negligible as are the higher terms in $\mathrm{B}^{2}$. The diffusive cooling term represents the tendency of the faster neutrons to leak from the system preferentially thereby cooling the energy distribution relative to a Maxwellian 


\begin{tabular}{|l|l|r|}
\hline & Canberra Industries, ACK\# D0043 & 33296 B \\
\hline Created: 29-September-2000 & IBIS Project & Revision B \\
\hline Revised: 13-January-2003 & Preliminary Design Study & Page 119 of 156 \\
\hline
\end{tabular}

distribution at the temperature of the moderator. For a system not isolated from its surroundings there can also be diffusive heating terms.

The geometrical buckling is defined as the lowest positive eigenvalue that results from solving the Helmholtz wave equation, Equation 23, for the neutron flux spatial distribution, $\phi(r)$, with the boundary conditions that the $\phi(r)$ is zero at the extrapolated boundary of the system. Because of the Cd wrapper, neutrons that leave the package do not return.

$$
\nabla^{2} \phi(r) / \phi(r)=B^{2}
$$

For a parallelepiped with extrapolated edges of length $\mathrm{a}(\mathrm{cm}), \mathrm{b}(\mathrm{cm})$ and $\mathrm{c}(\mathrm{cm})$ the solution of Eq 24 yields:

$$
B^{2}=\left(\frac{\pi}{a}\right)^{2}+\left(\frac{\pi}{b}\right)^{2}+\left(\frac{\pi}{c}\right)^{2}
$$

If the length of the body, $c$, is very much larger than either of the side lengths, $a$ and $b$, we immediately see that the geometrical buckling becomes insensitive to the length of the system. The extrapolated edge lengths are simply the true dimensions of the polyethylene block within the package with twice the extrapolation distance added. The extrapolation distance, $\mathrm{d}$, is in turn proportional to the transport mean free path, $\lambda \mathrm{t}$, for thermal neutrons in the medium The constant of proportionality depends on the theoretical model assumed and on the size and curvature of the surface of the body. In our case the exact value is not critical. We have chosen to adopt the estimator given by [Drozdowicz, et al., 1980] for hydrogenous media. For flat surfaces this becomes:

$$
d \approx 2.28 \cdot D
$$

where $\mathrm{D}$ is the diffusion coefficient. The diffusion coefficient $\mathrm{D}$ is numerically equal to one third of the transport mean free path. A commonly used expression for $\mathrm{D}$ in a modification of simple diffusion theory is [Bell and Glasstone, 1970].

$$
D \approx \frac{1}{3 \cdot\left[\Sigma_{a}+(1-\bar{\mu}) \cdot \Sigma_{s}\right]}
$$

where $\Sigma \mathrm{a} \quad$ is the macroscopic absorption cross section,

$\Sigma \mathrm{s} \quad$ is the macroscopic scattering cross section,

and $\bar{\mu}$ is the average cosine of the scattering angle in the laboratory frame. 


\begin{tabular}{|l|l|r|}
\hline & Canberra Industries, ACK\# D0043 & 33296 B \\
\hline Created: 29-September-2000 & IBIS Project & Revision B \\
\hline Revised: 13-January-2003 & Preliminary Design Study & Page 120 of 156 \\
\hline
\end{tabular}

If the scattering is isotropic in the center of momentum frame then, for non hydrogenous materials, we can approximate $\bar{\mu}$ as $2 /(3 . \mathrm{A})$, where $\mathrm{A}$ is the mass of the target nucleus in units of the neutron rest mass.

It is worth repeating that the expression we have developed for the thermal lifetime in the packages is the longest (dominant or fundamental mode) of a series of such time constants all of which are much shorter and which lead to transients that decay on time scales which are not of interest here. These harmonics are sometimes referred to as overtones.

The actual time profile of the active mode signal is a convolution of the induced fission profile and the intrinsic behavior of the package. In addition chamber and matrix dependent components may be evident due the hold-up of epi-Cd neutrons that can enter the FNDP walls. In behaving akin to a lead slowing down spectrometer this variable fast component which is present at short times after the pulse can mimic a fissile signal. In essence it contributes a variable background level which is difficult to quantify without careful analysis of the time profile. This requires excellent statistics and even then may require the time constants of the principle components to be fixed a priori in order to obtain a sensible fitted solution.

The simple analysis outlined above allows us to make several general statements about the design of FNDPs for DDA counting.

To minimize the intrinsic die-away time and maximize the neutron detection efficiency the package should be poisoned with ${ }^{3} \mathrm{He}$. In other words a high $3 \mathrm{He}$ pressure is favored and ${ }^{3} \mathrm{He}$ should form a significant component of the absorption cross section.

In order to minimize the intrinsic die-away time the geometrical bucking of the package should be high. This means that the package dimensions should be kept as small as possible commensurate with achieving an acceptable efficiency.

To avoid sensitivity to the thermal interrogating flux, and also to neutronically decouple the FNDP from its surrounding (and hence eliminate longer lived tails on the detector die-away profile caused by epi-Cd neutrons that have left the package returning later as thermal neutrons) the Cd wrapper around the FNDP's must be of generous thickness and free from weak spots. 


\begin{tabular}{|l|l|r|}
\hline & Canberra Industries, ACK\# D0043 & 33296 B \\
\hline Created: 29-September-2000 & IBIS Project & Revision B \\
\hline Revised: 13-January-2003 & Preliminary Design Study & Page 121 of 156 \\
\hline
\end{tabular}

\section{A.1. Calculations}

Thermal neutron diffusion parameters for low density $\left(\rho=0.92 \mathrm{~g} . \mathrm{cm}^{-3}\right)$ polyethylene at $20^{\circ} \mathrm{C}$ have been considered by Granada et al [Granada, et al., 1987]. For the present purposes these have been scaled to high density $\left(\rho=0.955 \mathrm{~g} . \mathrm{cm}^{-3}\right)$ polyethylene, which is used in practice, using the following scaling rules:

$$
\begin{aligned}
& v \cdot=\sum_{a} \propto \rho \text { Eq. } 27 \\
& \mathrm{D} \propto 1 / \rho \text { Eq. } 28 \\
& \mathrm{C} \propto 1 / \rho 3 \text { Eq. } 29
\end{aligned}
$$

With these adjustments we estimate:

$$
\begin{aligned}
& v \cdot \sum_{a}=\left(\frac{0.955}{0.920}\right) \cdot 5821=(6042 \pm 120) s^{-1} \\
& D=\left(\frac{0.920}{0.955}\right) \cdot\left(\frac{27133}{2.2 \times 10^{5}}\right)=(0.1188 \pm 0.0024) \mathrm{cm} \text { Eq. } 31 \\
& \mathrm{C}=\left(\frac{0.920}{0.955}\right)^{3} \cdot 2422=(2165 \pm 650) \mathrm{cm}^{4} \cdot \mathrm{s}^{-1} \text { Eq. } 32
\end{aligned}
$$

The uncertainties quoted are intended to be purely indicative but are representative of what typical good experimental determinations might be expected to return. They represent approximate $\pm 2 \%$ on (v. $\Sigma \mathrm{a}$ ) and $\mathrm{D}$ and $\pm 30 \%$ on C.

Diffusion parameters for ${ }^{3} \mathrm{He}$ gas at $20^{\circ} \mathrm{C}$ and $1 \mathrm{~atm}$ pressure; aluminum $\left(2.702 \mathrm{g.cm}{ }^{-3}\right)$ and stainless steel $\left(7.93{\mathrm{~g} . \mathrm{cm}^{-3}}^{-3}\right) \mathrm{Cr}(18 \mathrm{wt} \%), \mathrm{Mn}(1 \mathrm{wt} . \%)$, Fe (72 wt.\%), Ni (9 wt.\%)) were estimated using the formulae given above using data from the evaluated nuclear data libraries ENDF/B-6 and JEF-2.2 [ENDF/B-6]. Table 14. summarizes the values obtained. No attempt was made to estimate the diffusion cooling term coefficient for these materials.

In applying the model we make the approximation that the materials of the FNDP can be homogenized. Individual (v. $\Sigma a$ ) and D values are scaled according to their effective density within the package. For the package as a whole the (v. $\mathrm{a}$ ) and $\mathrm{D}$ value are obtained from those of the constituents (polyethylene, $\mathrm{Al}$ or $\mathrm{SS},{ }^{3} \mathrm{He}$ ) by combining the density adjusted values as follows: 


\begin{tabular}{|l|l|r|}
\hline & Canberra Industries, ACK\# D0043 & 33296 B \\
\hline Created: 29-September-2000 & IBIS Project & Revision B \\
\hline Revised: 13-January-2003 & Preliminary Design Study & Page 122 of 156 \\
\hline
\end{tabular}

$$
\begin{array}{lll}
(v \cdot \Sigma \mathrm{a}) & =\sum_{i}\left(v \cdot \sum_{a}\right)_{i} \text { Eq. } 33 \\
\frac{1}{D} & =\sum_{i} \frac{1}{D_{i}} \text { Eq. } 34
\end{array}
$$

where the summation, $\mathrm{i}$, runs over the constituents.

For the diffusion cooling term coefficient, $\mathrm{C}$, we have simply used the effective density scaled polyethylene value as the package value. This introduces a negligible uncertainty.

Table 14. Thermal neutron diffusion parameters for ${ }^{3} \mathrm{He}$ at $20^{\circ} \mathrm{C}$ and $1 \mathrm{~atm}$, aluminum and stainless steel.

\begin{tabular}{|l|l|l|}
\hline material & $\begin{array}{l}\text { v. } \Sigma \mathrm{a} \\
\left(\mathrm{s}^{-1}\right)\end{array}$ & $\begin{array}{l}\mathrm{D} \\
(\mathrm{cm})\end{array}$ \\
\hline $\begin{array}{l}{ }^{3} \mathrm{He} \\
\mathrm{Al}\end{array}$ & 29280 & 2.503 \\
$\mathrm{SS}$ & 3078 & 3.574 \\
\hline
\end{tabular}

We consider two basic FNDP designs, one for single ${ }^{3} \mathrm{He}$ proportional counters and one for an array of four ${ }^{3} \mathrm{He}$ proportional counters. Each can be based on the use of 1 " or 2 " O.D. tubes. The FNDP geometries are illustrated schematically in Figure 30 . In each case a ${ }^{3} \mathrm{He}$ tube of external radius, $\mathrm{R}$, and wall thickness, $\mathrm{w}$, is placed in a box of polyethylene defined by walls of thickness, t. There is a gap, $g$, between the tube and the polyethylene for clearance. According to this scheme the breadth of the four detector package is four times its width as it is simply constructed by stacking four of the single tube packages side by side. 


\begin{tabular}{|l|l|r|}
\hline & Canberra Industries, ACK\# D0043 & 33296 B \\
\hline Created: 29-September-2000 & IBIS Project & Revision B \\
\hline Revised: 13-January-2003 & Preliminary Design Study & Page 123 of 156 \\
\hline
\end{tabular}
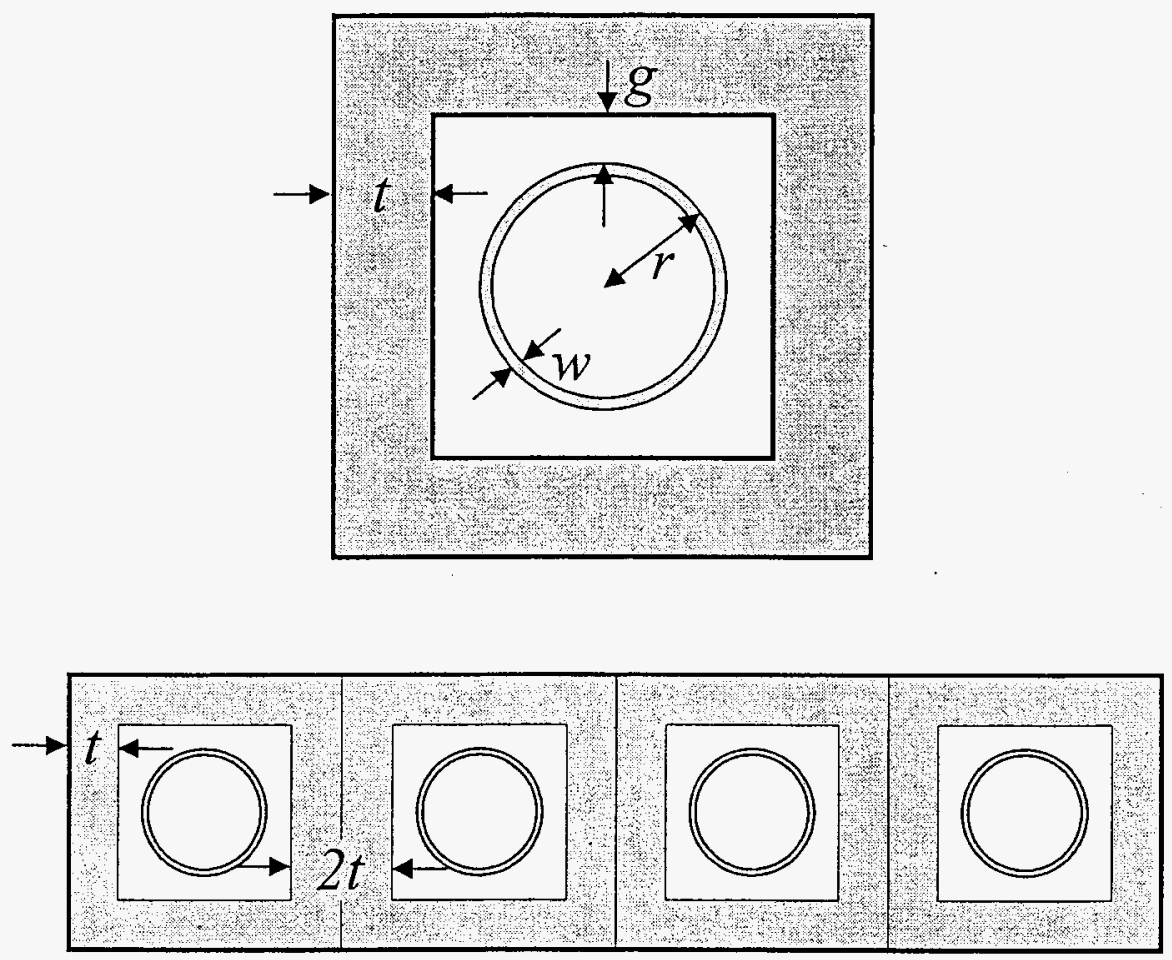

Fig. 30. Top illustration shows a single ${ }^{3} \mathrm{He}$ Tube FNDP The bottom illustration shows four ${ }^{3} \mathrm{He}$ Tube FNDP

\section{A.2. Results and Discussion}

The numerical calculations were carried out using a Microsoft Excel spreadsheet into which the nuclear data and formulae discussed above had been entered. Table 15 summarizes the results for several FNDP designs. In each case the length, c, of the package was fixed at $1000 \mathrm{~mm}$ and the gap, $\mathrm{g}$, between the ${ }^{3} \mathrm{He}$ tube and the polyethylene was set at $1.5 \mathrm{~mm}$. 


\begin{tabular}{|l|l|r|}
\hline & Canberra Industries, ACK\# D0043 & 33296 B \\
\hline Created: $29-$ September-2000 & IBIS Project & Revision B \\
\hline Revised: 13-January-2003 & Preliminary Design Study & Page 124 of 156 \\
\hline
\end{tabular}

Table 15. Estimates of the fundamental die-away time, $\tau$, for different FNDP designs.

\begin{tabular}{|c|c|c|c|c|}
\hline \multirow[t]{2}{*}{ Tube Type } & \multirow{2}{*}{$\begin{array}{l}{ }^{3} \mathrm{He} \text { fill } \\
\text { pressure } \\
\text { (atm) }\end{array}$} & \multirow{2}{*}{$\begin{array}{l}\text { Polyethylene } \\
\text { thickness } \\
\mathrm{t} \\
(\mathrm{mm})\end{array}$} & \multicolumn{2}{|c|}{ Decay time, $\tau(\mu \mathrm{s})$} \\
\hline & & & 1 tube FNDP & 4 tube FNDP \\
\hline \multirow[t]{2}{*}{$25.4 \mathrm{~mm}$ OD SS body } & 4 & $\begin{array}{l}12.5 \\
15 \\
19 \\
25\end{array}$ & $\begin{array}{l}24.9 \\
28.7 \\
35.0 \\
41.5\end{array}$ & $\begin{array}{l}29.7 \\
34.2 \\
44.6 \\
52.4\end{array}$ \\
\hline & 7.5 & $\begin{array}{l}12.5 \\
15 \\
19 \\
25\end{array}$ & $\begin{array}{l}17.6 \\
20.5 \\
25.4 \\
28.6\end{array}$ & $\begin{array}{l}19.9 \\
23.1 \\
33.1 \\
37.2\end{array}$ \\
\hline $25.4 \mathrm{~mm}$ OD Al body & $\begin{array}{l}4 \\
7.5 \\
\end{array}$ & $\begin{array}{l}12.5 \\
12.5 \\
\end{array}$ & $\begin{array}{l}26.4 \\
18.8 \\
\end{array}$ & $\begin{array}{l}31.8 \\
21.4 \\
\end{array}$ \\
\hline \multirow[t]{2}{*}{$50.8 \mathrm{~mm}$ OD SS body } & 2 & $\begin{array}{l}12.5 \\
15 \\
19 \\
25 \\
\end{array}$ & $\begin{array}{l}31.3 \\
34.5 \\
39.7 \\
47.4 \\
\end{array}$ & $\begin{array}{l}35.1 \\
38.6 \\
42.2 \\
52.6 \\
\end{array}$ \\
\hline & 4 & $\begin{array}{l}12.5 \\
15 \\
19 \\
25 \\
\end{array}$ & $\begin{array}{l}20.1 \\
22.3 \\
26.0 \\
31.8 \\
\end{array}$ & $\begin{array}{l}21.5 \\
23.9 \\
27.8 \\
34.2 \\
\end{array}$ \\
\hline $50.8 \mathrm{~mm}$ OD Al body & $\begin{array}{l}2 \\
4 \\
\end{array}$ & $\begin{array}{l}12.5 \\
12.5 \\
\end{array}$ & $\begin{array}{l}32.6 \\
20.8 \\
\end{array}$ & $\begin{array}{l}36.7 \\
22.3 \\
\end{array}$ \\
\hline
\end{tabular}

The ${ }^{3} \mathrm{He}$ like wall thickness was $0.4572 \mathrm{~mm}$ and $0.7112 \mathrm{~mm}$ in the case of $25.4 \mathrm{~mm}$ OD and $50.8 \mathrm{~mm}$ OD stainless steel bodies respectively. For aluminum walled tubes a thickness of $1 \mathrm{~mm}$ was used for both tube diameters. 


\begin{tabular}{|l|l|r|}
\hline & Canberra Industries, ACK\# D0043 & 33296 B \\
\hline Created: 29-September-2000 & IBIS Project & Revision B \\
\hline Revised: 13-January-2003 & Preliminary Design Study & Page 125 of 156 \\
\hline
\end{tabular}

These results illustrate that there is a small additional poisoning effect when stainless steel walled tubes are used rather than aluminum walled tubes. This effect on the fundamental die-away time is not sufficient for this to be a discriminate between the two choices.

The poisoning effect of using higher pressure ${ }^{3} \mathrm{He}$, although probably somewhat exaggerated in these calculations due to the homogenization process de-emphasizing the importance of shielding of the gas interior by the outer zones, is clearly visible. Because of this, use of $7.5 \mathrm{~atm}^{3} \mathrm{He}$ rather than $4 \mathrm{~atm}{ }^{3} \mathrm{He}$ in the case of $25.4 \mathrm{~mm}$ OD tubes and $4 \mathrm{~atm}^{3} \mathrm{He}$ rather than $2 \mathrm{~atm}{ }^{3} \mathrm{He}$ in the case of $50.8 \mathrm{~mm}$ OD tubes is preferred.

For a given class of FNDP (the 25.4mm OD 4 atm 1 tube pack for example) the trend for the dieaway time to increase as the package size (polyethylene wall thickness, $t$ ) increases is clearly revealed. This re-emphasizes that the package should be no larger than is required to achieve the target efficiency.

It is interesting to note that despite the difference in geometrical package size between the 1 tube and 4 tube packages and between the $25.4 \mathrm{~mm}$ OD and $50.8 \mathrm{~mm}$ OD versions, the absolute differences in the fundamental mode die-away times are not that great. Indeed from this prospective, there would seem to be little to choose between the $7.5 \mathrm{~atm}$ and $25.4 \mathrm{~mm}$ OD and the $4 \mathrm{~atm} 50.8 \mathrm{~mm}$ OD packs of a given polyethylene thickness. The selection criteria, therefore, centers on different issues: convenience of achieving a given spatial coverage of detection efficiency within the chamber; absolute detection efficiency; overload recovery properties and overall cost to achieve a given result being the prominent ones. 


\begin{tabular}{|l|l|r|}
\hline & Canberra Industries, ACK\# D0043 & 33296 B \\
\hline Created: 29-September-2000 & IBIS Project & Revision B \\
\hline Revised: 13-January-2003 & Preliminary Design Study & Page 126 of 156 \\
\hline
\end{tabular}

\section{B. Measured Response of the CWAM System}

An induced fission reaction takes place at a rate that is directly proportional to both the total number of fissile nuclei and the interrogating scalar neutron flux. For neutrons with a fixed energy, the reaction rate density is given by

$$
R R_{f}=n(r) \cdot v \Sigma_{f}
$$

where

$$
\begin{aligned}
\mathrm{RR}_{\mathrm{f}}= & \text { number of reactions per } \mathrm{cm}^{3} \text { per s }\left(\mathrm{cm}^{-3} \mathrm{~s}^{-1}\right), \\
\mathrm{n}(\mathrm{r})= & \text { neutron number density at position } \mathrm{r}\left(\mathrm{cm}^{-3}\right) \text {, and } \\
\mathrm{v} \Sigma_{\mathrm{f}}= & \text { fission interaction frequency }\left(\mathrm{s}^{-1}\right) . \text { For a spectrum of neutron energies the } \\
& \text { interaction frequency is obtained from the spectrum weighted average speed } \\
& \text { microscopic cross section product }- \text { the form of the expressions in this section } \\
& \text { remain unchanged. }
\end{aligned}
$$

If the fissile material contains only ${ }^{235} \mathrm{U}$, the total number of prompt fission neutrons produced in the reaction is given by

$$
N_{f}=\bar{v} \cdot N_{235} \cdot \sigma_{f} \cdot \Phi,
$$

where

$$
\begin{aligned}
& N_{\mathrm{f}}=\text { number of fission neutrons produced per second }\left(\mathrm{s}^{-1}\right), \\
& \bar{v}=\text { average number of prompt neutrons released per fission, } \\
& \sigma_{\mathrm{f}}=\text { spectrum-weighted average fission microscopic cross section for }{ }^{235} \mathrm{U}\left(\mathrm{cm}^{2}\right), \\
& \Phi=v \mathrm{n}(\mathrm{r}) ; \text { Scalar neutron flux }\left(\mathrm{cm}^{-2} \mathrm{~s}^{-1}\right)
\end{aligned}
$$

and

$$
\begin{aligned}
& \mathrm{N}_{235}=\frac{\mathrm{M}_{235} \cdot \mathrm{N}_{\mathrm{A}}}{\mathrm{A}} \\
& \mathrm{N}_{235}=\text { number of }{ }^{235} \mathrm{U} \text { atoms in the fissile material, } \\
& \mathrm{N}_{\mathrm{A}}=\text { Avogadro's number, } 6.02322 \times 10^{23} \text { atoms } \mathrm{mol}^{-1} \text {, and } \\
& \mathrm{A}=\text { atomic weight }\left(\mathrm{g} \mathrm{mol}^{-1}\right) .
\end{aligned}
$$

Solving for the mass, 


\begin{tabular}{|l|l|r|}
\hline & Canberra Industries, ACK\# D0043 & 33296 B \\
\hline Created: 29-September-2000 & IBIS Project & Revision B \\
\hline Revised: 13-January-2003 & Preliminary Design Study & Page 127 of 156 \\
\hline
\end{tabular}

$$
M_{235}=\left(\frac{A}{v \cdot N_{A} \cdot \sigma_{f}}\right) \cdot \frac{N_{f}}{\Phi}
$$

Note in this equation that $\mathrm{N}_{\mathrm{f}}$ is proportional to the net detected signal. Grouping constants and solving for the active calibration factor, we obtain the following equation in terms of the specific measurement parameters

$$
\mathrm{K}_{\mathrm{A}}=\frac{\left(\mathrm{M}_{235} * \mathrm{SSF}\right)}{(\mathrm{ANS}-\mathrm{ANB})}
$$

where

$$
\begin{aligned}
& \mathrm{M}_{235}=\text { uranium-235 mass, } \\
& \mathrm{SSF}=\mathrm{MCNP} \mathrm{TM}_{\mathrm{TM}} \text {-calculated self-shielding factor, } \\
& \mathrm{ANS}=\text { active net fissile signal-measured with source, } \\
& \mathrm{ANB}=\text { active net blank signal-measured with no source. }
\end{aligned}
$$

The active net fissile and blank signal are calculated using the same equation given by

$$
\text { ANS }=\frac{\left(C_{i}-g * B_{i}\right)}{F_{i}}
$$

or

$$
\operatorname{ANB}=\frac{\left(C_{i}-g * B_{i}\right)}{F_{i}}
$$

where,

$\mathrm{g}=$ ratio of gate lengths for count and background windows,

$\mathrm{C}_{\mathrm{i}}=$ total integrated counts in shielded detectors during $850-2,850 \mu \mathrm{s}$,

$\mathrm{B}_{\mathrm{i}}=$ total integrated counts in shielded detectors during $14-18 \mathrm{~ms}$,

$F_{i}=$ total integrated counts in bare flux monitors during $850-2,850 \mu \mathrm{s}$.

Note also $B_{i}$ is the ambient background and source-related background including any delayed neutron components. By measuring a known standard, Equation 39 can be used to determine the active calibration factor, $K_{A}\left[g\left({ }^{235} \mathrm{U}\right) /\right.$ normalized net signal] for the CWAM system. In DDT 


\begin{tabular}{|l|l|r|}
\hline & Canberra Industries, ACK\# D0043 & 33296 B \\
\hline Created: 29-September-2000 & IBIS Project & Revision B \\
\hline Revised: 13-January-2003 & Preliminary Design Study & Page 128 of 156 \\
\hline
\end{tabular}

waste assay, there are two general types of calibrations that may be performed: 1) wide-range calibration and 2) matrix-specific calibration. Although the wide-range calibration method is appealing, it can lead to large errors when an unknown matrix doesn't fit the criteria assumed in the analysis. In the case of the drum-sized CTEN, a total of 43 matrices were measured providing a comprehensive database from which to derive corrections. The practicalities of measuring crates in the limited time available for the calibration work at Los Alamos resulted in only four matrices being measured. Without additional data (more surrogates or MCNPTM modeling) the amount of cases was insufficient for the development of wide-ranging matrix corrections.

If the waste matrices are well known, an improved accuracy may be obtained by determining calibration constants that are matrix-specific. The matrix-specific calibration assumes a matrix type and calibrates using a mock-up of the matrix crate. Since matrix properties of the calibration surrogate are assumed equal to the unknown, theoretically no matrix corrections are needed. The Oak Ridge Y-12 Plant has at least two such matrices in their inventory, scrap metal and baled waste. Of these two inventory matrices, only a surrogate for the scrap metal matrix was available at the time of the calibration work at Los Alamos. In addition to the metallic matrix, three surrogate matrices with varying hydrogen densities were also measured to simulate combustibletype waste. Canberra has since purchased two more surrogates, a baled waste and an additional scrap metal matrix that are in the process of being incorporated into the CWAM measurement database.

Although matrix-specific calibration factors were determined for each of the surrogate matrices, additional data was taken to study the effects of matrix on interrogating flux and fissile signal. The goal of the matrix characterizations study was to determine the best measured or calculated indicators of the following:

- Interrogating Flux Generation and Decay

- Fissile Signal Transmission

- Position of Source Material

These general categories served as organizing principles in the creation of the databases for each of the matrices. Although only scaler data was necessary to determine matrix specific calibration factors, list mode data was also taken using the CAMAC-based PATRM module in both the active and passive modes. Independent measurements of the flux in the active and transmission in the passive were performed as a function of 72 positions within the surrogate matrices. This data proved useful in benchmarking the MCNPTM model for the neutron assay system of the IBIS.

The expression in Equation 38 is not strictly valid for the measurement of ${ }^{235} \mathrm{U}$ in a crate in the CWAM system. Both the intensity of the interrogating flux and the transmission of the induced fission neutrons varies with matrix type and source position. Before flux-and transmissionrelated corrections can be developed for an unknown matrix, the relationships between the 


\begin{tabular}{|l|l|r|}
\hline & Canberra Industries, ACK\# D0043 & 33296 B \\
\hline Created: $29-$ September-2000 & IBIS Project & Revision B \\
\hline Revised: 13-January-2003 & Preliminary Design Study & Page 129 of 156 \\
\hline
\end{tabular}

variables $\Phi$ and $\mathrm{N}_{\mathrm{f}}$ and the comparable responses in CWAM need to be well understood. The sum of the responses from the three bare assay chamber monitors from $850-2,850 \mu$ s is used to measure the intensity of the interrogating thermal flux. However, these detectors measure a crateaveraged response that does not necessarily indicate the thermal neutron density at the fissile material location.

Figure 31 compares the response of a bare flux monitor located at the fissile source location (72 different positions) to that of the crate average responses. The straight lines represent the average of 72 flux measurements of the assay chamber flux monitors (located on the left, right, and back walls) for each of the matrices. The open diamonds represents the flux measured at each of 72 positions within the matrix. Measurements were performed for an interrogation window of 850 to $2,850 \mu$ s at 40,000 pulses ( 4 generator positions). As expected the flux is uniform throughout the empty crate and is essentially equal to the response measured by the chamber flux monitors (within 1\%).

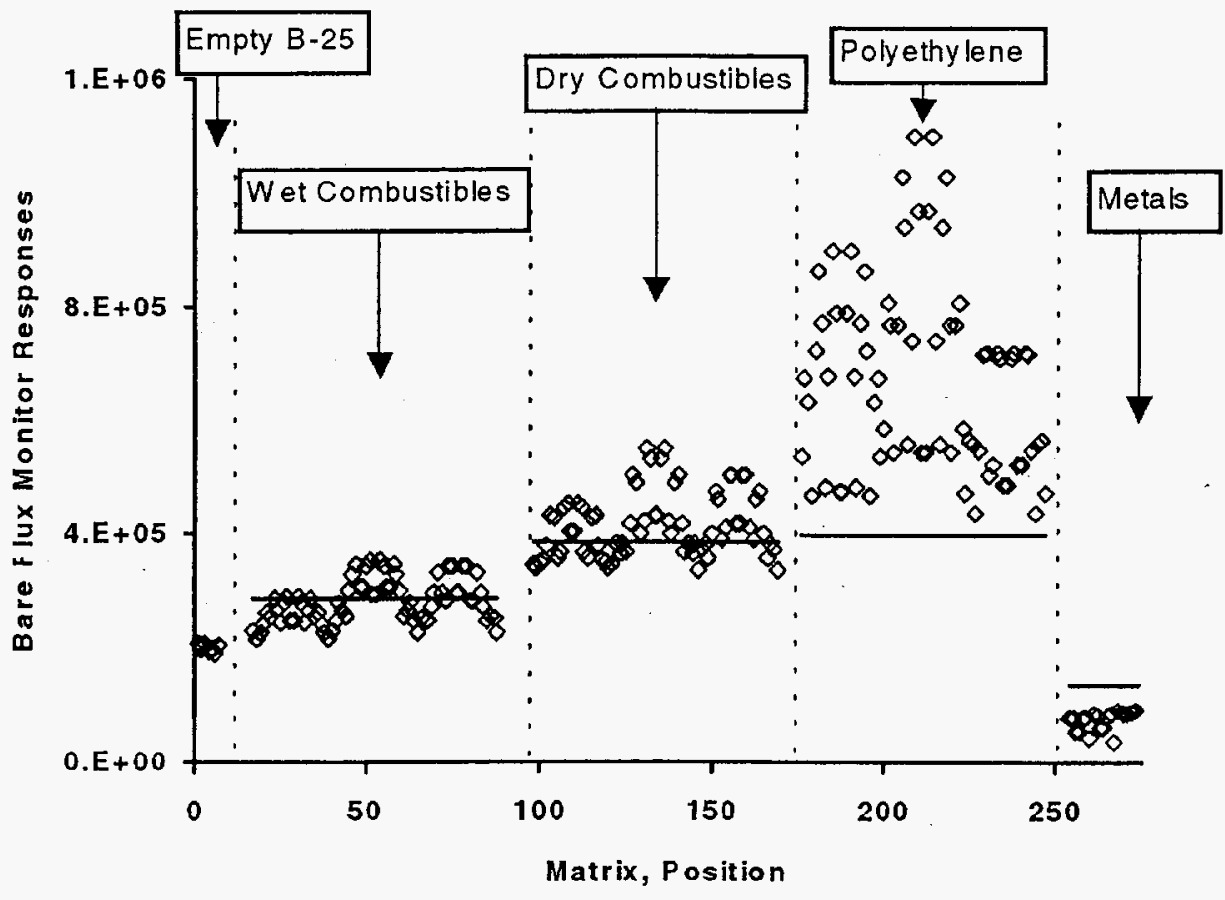

Figure 31. Responses of bare flux monitors, both internal and external to the matrix as a function of position and matrix for the thermal region.

Both the wet and dry combustible matrices contain the same absorber content but different in their hydrogen densities $\left(0.021 \mathrm{~g} \mathrm{~cm}^{-3}\right.$ and $0.016 \mathrm{~g} \mathrm{~cm}^{-3}$, respectively). The ratio of absorber to moderator in the wet combustibles resulted in an interior flux average comparable to that measured externally. Interior flux averaged over the 72 positions in the wet combustibles is 280,733 counts for a standard 4-position assay with a relative standard deviation of $15 \%$. This 


\begin{tabular}{|l|l|r|}
\hline & Canberra Industries, ACK\# D0043 & 33296 B \\
\hline Created: 29-September-2000 & IBIS Project & Revision B \\
\hline Revised: 13-January-2003 & Preliminary Design Study & Page 130 of 156 \\
\hline
\end{tabular}

compares well with the assay chamber monitors with a count of 287,958 for the same measurement parameters (within 3\%).

The flux inside the dry combustibles is still fairly well represented by the chamber monitors, being underestimated by $7 \%$ with a relative standard deviation of $14 \%$. In comparison, the flux in the polyethylene matrix is underestimated by over $70 \%$. Spatial variation of the flux in the polyethylene matrix ( $\rho_{\mathrm{H}}=0.02 \mathrm{~g} \mathrm{~cm}^{-3}$ and no substantial absorbers) has a relative standard deviation of $26 \%$. In contrast, flux in a matrix containing little moderating ability, such as iron, will be overestimated by the chamber monitors. In this case, the interior flux is on average $45 \%$ lower than what is measured by the chamber monitors.

Likewise, the integrated net responses of the shielded detectors from $850-2,850 \mu$ s measure only those neutrons arriving at the shielded detectors with energies above the filter cutoff. Thus the measured response of the shielded detectors is always less than the actual number of neutrons produced in the fissile material. Transmission of fission neutrons from a plutonium source was calculated from passive data taken at 72 positions within each of the surrogate matrices. The standard used in the passive assay was a $100-\mathrm{g}$ plutonium metal cylinder with well-known source strength and multiplication values.

Figure 32 shows the transmission as a function matrix type and source position. Nonhydrogenous matrices such as iron and the empty B-25 have transmission values greater than $95 \%$. Hydrogen containing matrices have transmission values varying from 15 to $50 \%$ depending on position and hydrogen content. For matrices containing hydrogen, the minimum transmission values occur in the center of the matrix. See Table 16 for the minimum, maximum, and average transmission values for the 72 positions within each of the matrices. 


\begin{tabular}{|l|l|r|}
\hline & Canberra Industries, ACK\# D0043 & 33296 B \\
\hline Created: 29-September-2000 & IBIS Project & Revision B \\
\hline Revised: 13-January-2003 & Preliminary Design Study & Page 131 of 156 \\
\hline
\end{tabular}

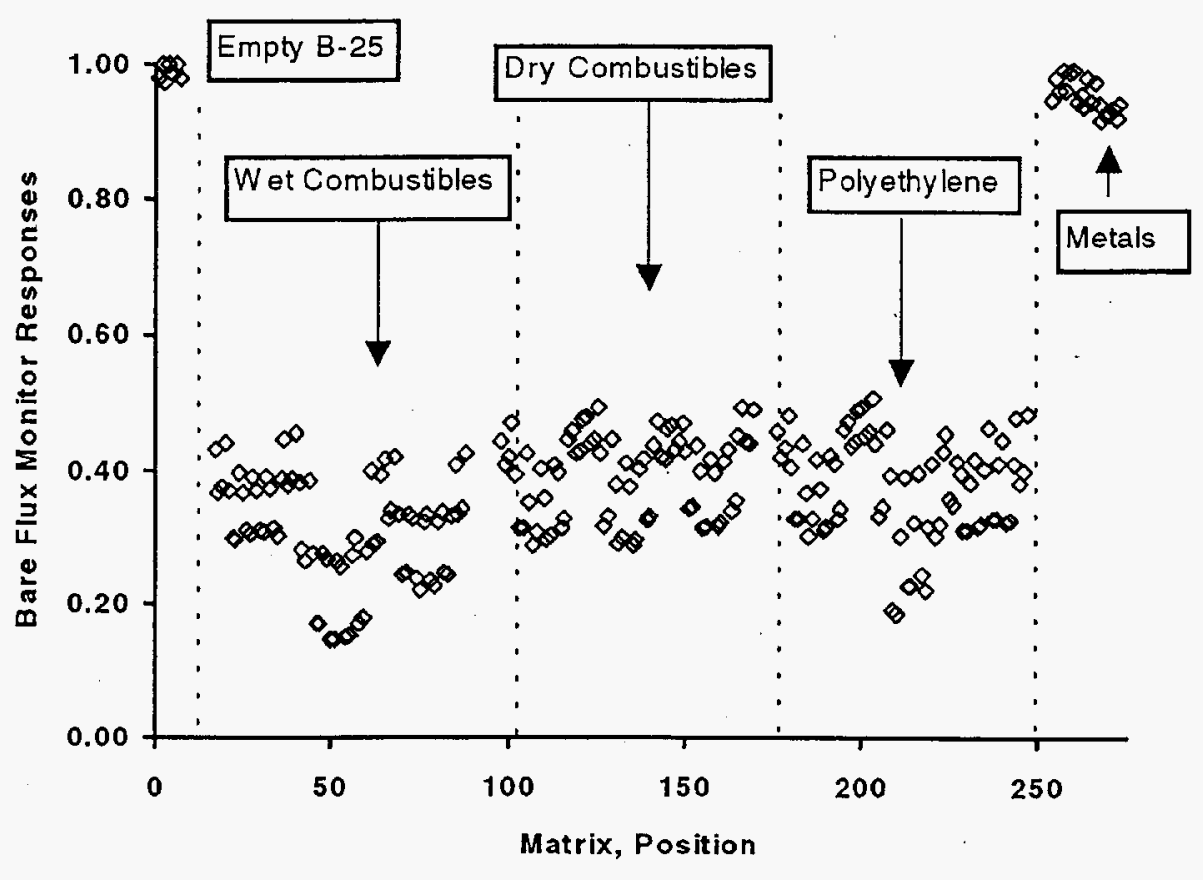

Figure 32. Transmission of fission neutrons as a function of position and matrix as measured using a 100-g plutonium metal cylinder.

The detection efficiency of the shielded detector packages in CWAM is 14\% for fission spectrum neutrons located at the center of the empty chamber. For the center of the wet combustibles matrix ( $\rho_{\mathrm{H}}=0.021 \mathrm{~g} \mathrm{~cm}^{-3}$ ), only $15 \%$ of the fission neutrons are transmitted through the matrix. This corresponds to $2.1 \%$ detection efficiency for single neutrons located at the matrix center. Although this represents a loss of signal neutrons, the interrogating thermal flux is enhanced at this position (relative to the empty crate) increasing the fission probability. 


\begin{tabular}{|l|l|r|}
\hline & Canberra Industries, ACK\# D0043 & 33296 B \\
\hline Created: 29-September-2000 & IBIS Project & Revision B \\
\hline Revised: 13-January-2003 & Preliminary Design Study & Page 132 of 156 \\
\hline
\end{tabular}

Table 16. Minimum, maximum, and average values of the measured transmission for 72 positions within the crate.

\begin{tabular}{|l|c|c|c|}
\hline \multicolumn{1}{|c|}{$\begin{array}{c}\text { Surrogate } \\
\text { Matrix }\end{array}$} & \multicolumn{3}{|c|}{ Fraction of Fission Neutrons Transmitted } \\
\hline & $\begin{array}{c}\text { Minimum } \\
(\%)\end{array}$ & $\begin{array}{c}\text { Maximum } \\
(\%)\end{array}$ & $\begin{array}{c}\text { Average } \\
(\%)\end{array}$ \\
\hline Empty B25 & 97 & 100 & 99 \\
\hline Scrap Metal-B25 & 92 & 99 & 95 \\
\hline $\begin{array}{l}\text { Dry Combustibles- } \\
\text { SWB }\end{array}$ & 29 & 49 & 39 \\
\hline Polyethylene-B25 & 19 & 51 & 38 \\
\hline Wet Combustibles-B25 & 15 & 46 & 31 \\
\hline
\end{tabular}

In comparing fissile signal loss in Table 16 to the thermal flux gain of Figure 31 , the polyethylene matrix shows a net gain. This is also shown graphically in Figure 33 that shows the net fissile signal for a $368 \mathrm{~g}$ depleted uranium metal bar (with an apparent mass of $625.6 \mathrm{mg}$ ${ }^{235} \mathrm{U}$ ) as a function matrix type and source position. The net fissile signal in this case is the net number of neutrons detected during the thermal region $(850-2,850 \mu$ s), i.e. background subtracted using the time region of 14-18 ms. From this figure, one can see that the sensitivity in a large number of locations within the polyethylene matrix actually improves relative to the empty crate.

Since the hydrogen content of each matrix varies, its effect on the interrogating flux will also vary. A matrix containing hydrogen with little or no absorber will have an increased thermal flux in its interior, thereby increasing the fission rate. If this were the only effect on the assay, the sensitivity would be improved relative to the empty crate. Neutrons from induced-fission are also moderated by the hydrogen in the matrix. As the density of hydrogen increases and the source material extends further into the matrix, the chance is decreased that a fission neutron will reach the shielded detectors with energies sufficient to pass the cadmium barrier. Thus, the apparent detector efficiency is reduced and the sensitivity is decreased. Thus, these two effects of a purely moderating matrix are directly opposed.

At some combination of hydrogen density and depth within the matrix, the two effects of moderation should approximately cancel. This is seen in some locations within the polyethylene matrix where the sensitivity is equal to the case of the empty crate. Although moderation due to hydrogen is the major interference for an active measurement, absorbers may also cause severe problems. The probability of an interaction of a neutron with a nucleus is increased as the neutron approaches thermal energies. If the matrix contains highly absorbing materials, the thermal flux will be suppressed. The fission rate will decrease and the sensitivity will be reduced. Since the absorption cross section for fission spectrum neutrons (average energy of $\sim 2 \mathrm{MeV}$ ) is relatively low, pure absorbers have little effect on the fission neutrons. However, the addition of 


\begin{tabular}{l}
\hline \\
$\qquad$\begin{tabular}{|l|l|r|}
\hline & Canberra Industries, ACK\# D0043 & 33296 B \\
\hline Created: 29-September-2000 & IBIS Project & Revision B \\
\hline Revised: 13-January-2003 & Preliminary Design Study & Page 133 of 156 \\
\hline
\end{tabular}
\end{tabular}

a substantial absorber to a highly moderating matrix will further reduce the number of fission neutrons detected as seen in the comparison of the polyethylene and wet combustibles matrices.

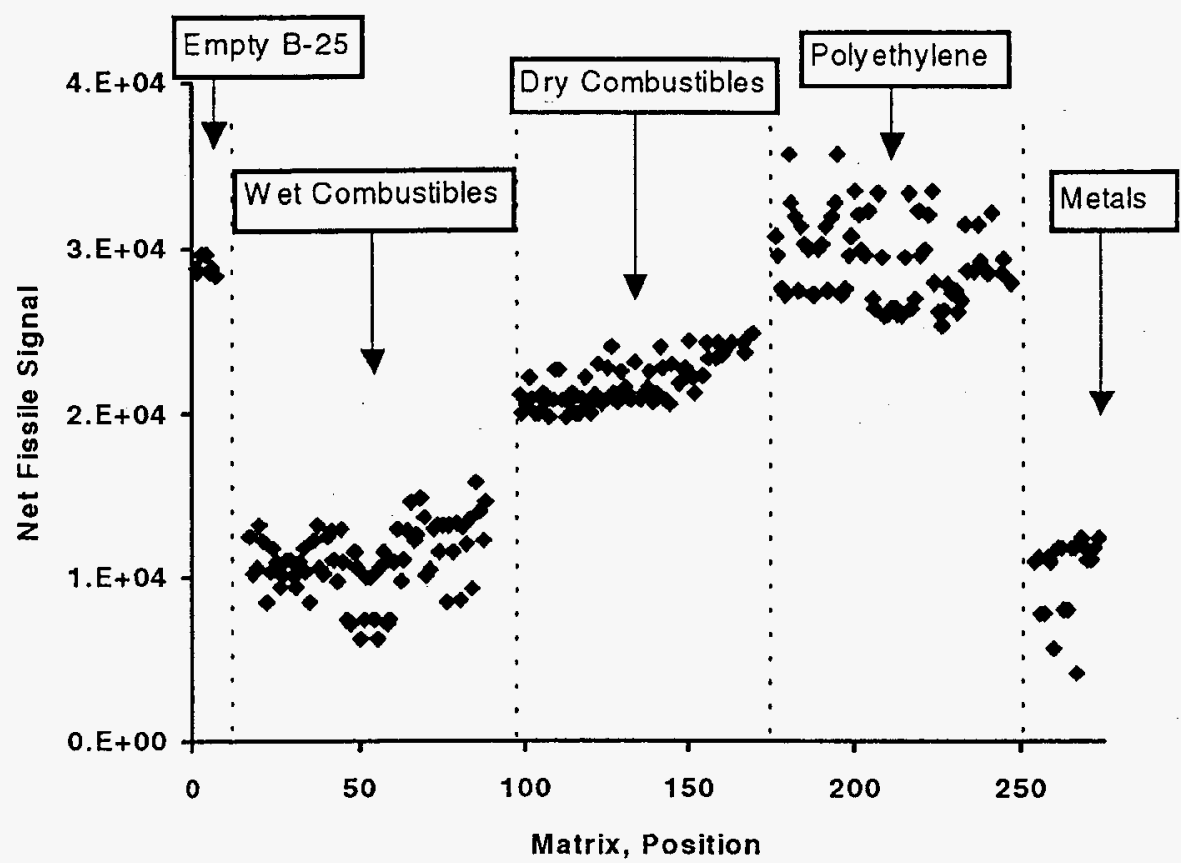

Figure 33. Net fissile signal in the thermal interrogation region for a $625.6 \mathrm{mg}{ }^{235} \mathrm{U}$ source as a function of matrix and position.

The relative standard deviation of the net fissile signal for all the matrices in Figure 33 is $41 \%$. Figure 34 shows the "corrected" net signal using the measured transmission and flux values for each of the 72 positions. The relative standard deviation for this corrected value drops to $12 \%$. Of course, in actual waste the true interrogating flux and transmission values are unknown.

Corrections for interrogating flux and transmission can only be estimated from data that can be taken during the assay. For actual waste matrices, the net signal is normalized using the crateaverage responses from the assay chamber flux monitors. As seen in Figure 31, this value may overestimate or underestimate the true flux. Currently there are no measured indicators of transmission. 


\begin{tabular}{|l|l|r|}
\hline & Canberra Industries, ACK\# D0043 & 33296 B \\
\hline Created: 29-September-2000 & IBIS Project & Revision B \\
\hline Revised: 13-January-2003 & Preliminary Design Study & Page 134 of 156 \\
\hline
\end{tabular}

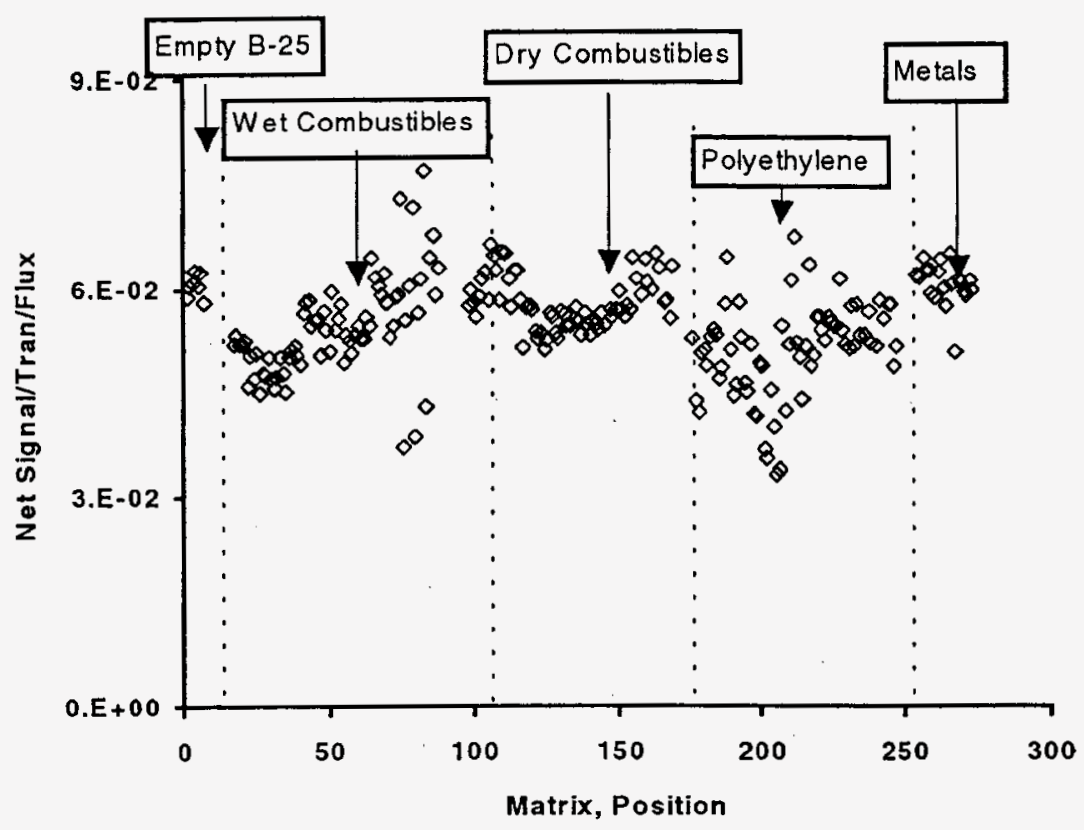

Figure 34. Net fissile signal corrected using the measured flux and transmission values for that position.

\section{B.1 Active Measurements the in CWAM System}

As a first approximation, the number of induced fission in a DDT assay is proportional to the interrogating thermal flux and the concentration of ${ }^{235} \mathrm{U}$ in the waste crate. By measuring prompt neutrons from the thermal interrogation region, sensitivities of a few milligrams of fissile mass are attained in a relatively short time. However, the degree of accuracy required to meet performance objectives usually dictates the use of corrections to account for the effects of matrix type and source distribution on the measured response.

The ability to interrogate from more than one position in a DDT assay improves the overall accuracy by providing a more uniform flux profile. In CTEN, the neutron generator is located in the back left corner and the drum makes one revolution during the entire assay. In CWAM, multiple interrogations are performed at predetermined stops along a motorized track located on the back wall of the assay chamber. A complete assay is the sum of the individual interrogations. Figure 35 shows the various neutron generator positions schemes tested during the matrix characterization study. Positions $\mathrm{A}, \mathrm{B}$, and $\mathrm{C}$ are designated back right, back center, and back. left, respectively. Positions D, E, and F result from turning the crate around and interrogating on the other side. The optimal measurement scheme is the one that minimizes the variation of the fissile signal as a function of source position. 


$\mid$\begin{tabular}{|l|l|r|}
\hline & Canberra Industries, ACK\# D0043 & 33296 B \\
\hline Created: $29-$ September-2000 & IBIS Project & Revision B \\
\hline Revised: 13-January-2003 & Preliminary Design Study & Page 135 of 156 \\
\hline
\end{tabular}

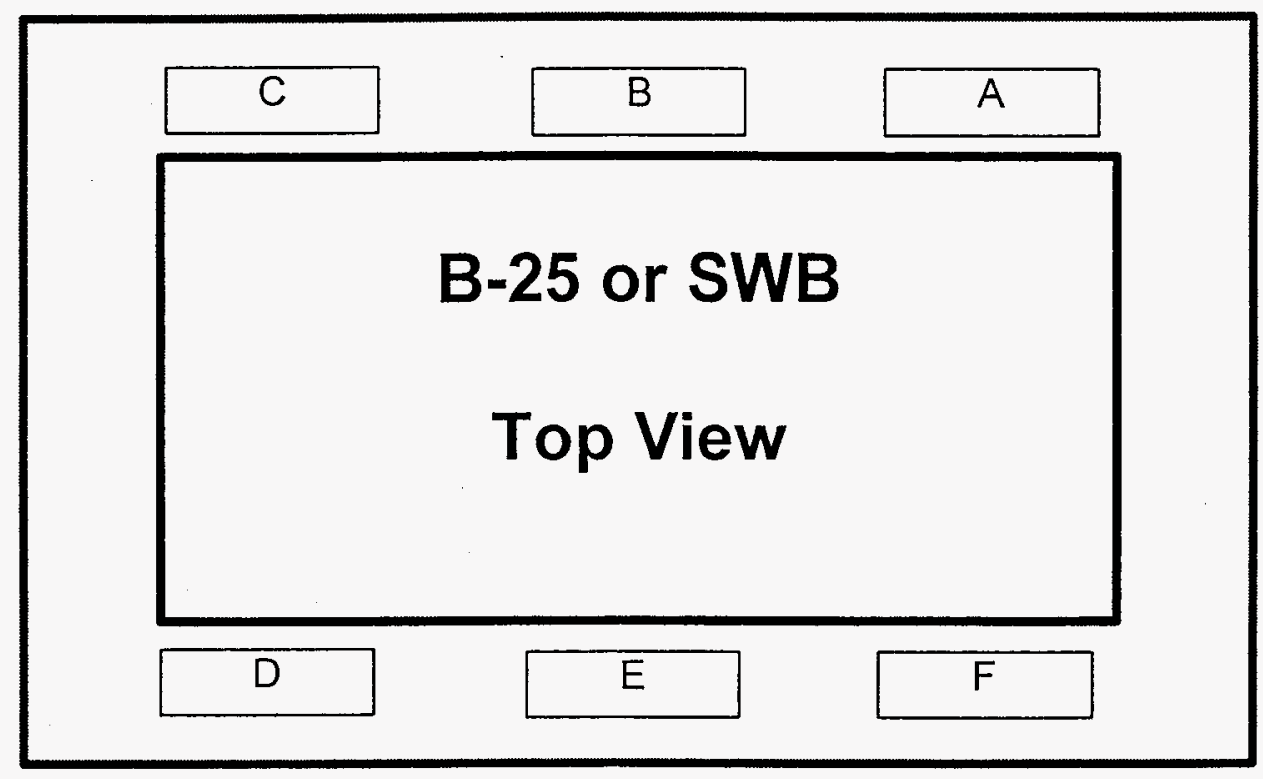

Figure 35. Neutron generator measurement schemes tested during calibration study.

Table 17 shows the spatial variation over 72 positions in each of the surrogate matrices for the five measurement schemes tested. The source used was a depleted uranium metal bar with an apparent ${ }^{235} \mathrm{U}$ mass of $625.6 \mathrm{mg}$ (corrected for self shielding). The generator was pulsed at $50 \mathrm{~Hz}$ for 10,000 pulses per position. Although spatial variation is greatest for the hydrogen-containing matrices, the ability to interrogate on both sides of the crate has a significant effect. The optimal configuration was obtained with four positions in which the crate is rotated and interrogated on the back left and right positions. Assay time is 800 seconds with four neutron generator stops at 10,000 pulses per stop. Total measurement time including opening and closing the door, moving the loading platform, and operating the forklift is approximately 50 minutes. 


\begin{tabular}{|l|l|r|}
\hline & Canberra Industries, ACK\# D0043 & 33296 B \\
\hline Created: 29-September-2000 & IBIS Project & Revision B \\
\hline Revised: 13-January-2003 & Preliminary Design Study & Page 136 of 156 \\
\hline
\end{tabular}

Table 17. Variation of fissile signal over 72 positions for five measurement schemes.

\begin{tabular}{|c|c|c|c|c|c|}
\hline \multirow{2}{*}{$\begin{array}{l}\text { Number } \\
\text { of Stops }\end{array}$} & \multirow{2}{*}{$\begin{array}{l}\text { Generator } \\
\text { Positions }\end{array}$} & \multicolumn{4}{|c|}{$\begin{array}{c}\text { Positional Variation } \\
\text { (RSD\%) }\end{array}$} \\
\hline & & $\begin{array}{c}\text { Dry } \\
\text { Combustibles }\end{array}$ & $\begin{array}{c}\text { Wet } \\
\text { Combustible } \\
\text { s }\end{array}$ & $\begin{array}{l}\text { Polyethylene } \\
\text { B25 }\end{array}$ & $\begin{array}{c}\text { Carbon } \\
\text { Steel }\end{array}$ \\
\hline 1 & B & 27 & 37 & 31 & 26 \\
\hline 2 & $\mathrm{AC}$ & 21 & 38 & 29 & 25 \\
\hline 3 & $A B C$ & 22 & 35 & 27 & 25 \\
\hline 4 & $\mathrm{AC} \& \mathrm{DF}$ & 8 & 17 & 11 & 22 \\
\hline 6 & (ALL) & 12 & 18 & 16 & 22 \\
\hline
\end{tabular}

\section{B.2 Sensitivity for the Active Assay}

A commonly used definition of sensitivity or minimum detectable mass in regards to nondestructive assay systems is the smallest mass that gives a signal that is three times its uncertainty. The confidence interval is chosen as $95 \%$ or $99 \%$ representing signals that are two sigma and three sigma above background, respectively. [Kunz, Atencio, and Caldwell, 1980; Nicholas, Cooper, and Estep, 1992; Rinard, et al., 1992; Crane, 1984]. Using these definitions, the sensitivity in an empty chamber for the standard four-position assay (40,000 pulses) is 7.7 and $11.5 \mathrm{mg}$ of ${ }^{235} \mathrm{U}$. Since the active response is uniform for the empty chamber, the sensitivity can be stated as one mass value. For crates containing matrix materials, the sensitivity varies with matrix type and position of the source material. Table 18 shows the " 3 sigma over background" limits for the best, worse, and average positions within each of the matrices. The best and worse positions are necessarily point source results while the average sensitivity represents a uniform distribution. These sensitivity limits are based on equivalent observations of the "signal plus blank "and the blank. The reporting interval of 2 sigma over background ( $95 \%$ confidence interval) is shown in Table 19. Note for simple scaling purposes the crate average is given approximately by ( $3 \times$ Best $+1 \times$ Worst) $/ 4$. 


\begin{tabular}{|l|l|r|}
\hline & Canberra Industries, ACK\# D0043 & 33296 B \\
\hline Created: 29-September-2000 & IBIS Project & Revision B \\
\hline Revised: 13-January-2003 & Preliminary Design Study & Page 137 of 156 \\
\hline
\end{tabular}

Table 18. Three sigma over background sensitivity in ${ }^{235} \mathrm{U}$ mass for the best, worse, and average positions. (99\%Cl)

\begin{tabular}{|l|c|c|c|c|}
\hline $\begin{array}{c}\text { Surrogate } \\
\text { Matrix }\end{array}$ & $\begin{array}{c}\text { Best Position } \\
\left(\mathbf{m g}{ }^{235} \mathbf{U}\right)\end{array}$ & $\begin{array}{c}\text { Worse Position } \\
\left(\mathbf{m g}^{\mathbf{2 3 5}} \mathbf{U}\right)\end{array}$ & $\begin{array}{c}\text { Crate Average } \\
\left(\mathbf{m g}{ }^{\mathbf{2 3 5}} \mathbf{U}\right)\end{array}$ & $\begin{array}{c}\text { Max } \\
\mathbf{M i n}\end{array}$ \\
\hline Empty B-25 & - & - & 11.5 & $\sim 1$ \\
\hline Empty SWB & - & - & 10.3 & $\sim 1$ \\
\hline Dry Combustibles & 10.7 & 18.2 & 13.7 & 1.7 \\
\hline Polyethylene & 8.0 & 16.6 & 10.0 & 2.1 \\
\hline Scrap Metal & 24.3 & 72.7 & 29.7 & 3.0 \\
\hline Wet Combustibles & 14.8 & 45.1 & 25.5 & 3.0 \\
\hline
\end{tabular}

Table 19. Two sigma over background sensitivity in ${ }^{235} \mathrm{U}$ mass for the best, worse, and average positions. $(95 \% \mathrm{CI})$

\begin{tabular}{|l|c|c|c|}
\hline $\begin{array}{c}\text { Surrogate } \\
\text { Matrix }\end{array}$ & $\begin{array}{c}\text { Best Position } \\
\left(\mathbf{m g}{ }^{235} \mathbf{U}\right)\end{array}$ & $\begin{array}{c}\text { Worse Position } \\
\left(\mathbf{m g}{ }^{\mathbf{2 3 5}} \mathbf{U}\right)\end{array}$ & $\begin{array}{c}\text { Crate Average } \\
\left(\mathbf{m g}{ }^{\mathbf{2 3 5}} \mathbf{U}\right)\end{array}$ \\
\hline Empty B-25 & - & - & 7.7 \\
\hline Empty SWB & - & - & 6.8 \\
\hline Dry Combustibles & 7.1 & 12.1 & 9.1 \\
\hline Polyethylene & 5.4 & 11.1 & 6.7 \\
\hline Scrap Metal & 16.2 & 48.5 & 19.8 \\
\hline Wet Combustibles & 9.88 & 30.1 & 16.9 \\
\hline
\end{tabular}

\section{B.3 Sensitivity According to Currie}

Although the sensitivity for nondestructive waste assay systems is generally quoted as three sigma over background, the more widely accepted approach is based on Currie's 1968 paper, "Limits for Qualitative Detection and Quantitative Determination." [Currie, 1968] A Martin Marietta Energy Systems position paper entitled "Standardized Statistical Techniques for Radiological Measurements" published in 1993 was strongly based on the Currie's statistical techniques. [Martin Marietta, 1993] In the position paper, two limiting levels were defined:

1. Decision Level or Critical Level $\left(\mathrm{L}_{\mathrm{C}}\right)$-the net signal level above which an observed signal may be reliably recognized as detected,

2. Detection Limit or Lower Limit of Detection (LLD) or Minimum Detectable Activity (MDA)-the lowest practical quantity that the instrument can routinely detect. "Routine 


\begin{tabular}{|l|l|r|}
\hline & Canberra Industries, ACK\# D0043 & 33296 B \\
\hline Created: 29-September-2000 & IBIS Project & Revision B \\
\hline Revised: 13-January-2003 & Preliminary Design Study & Page 138 of 156 \\
\hline
\end{tabular}

detection means that if the activity is equal to the detection limit, the instrument will report a detected result at least $95 \%$ of the time, but not necessarily equal to the detection limit."

For the $95 \%$ confidence level for one-tailed statistics, the probability of incorrect positive assumption (Type I error, $\alpha$ ) and a incorrect non-detection (Type II error, $\beta$ ) are set equal to 0.05 . This is the standard adopted by Energy Systems. The decision or critical level equation is based on the maximum allowable value for $\alpha$ and the standard deviation of the net signal when the its limiting mean is zero. The equation for the decision level is given by:

$$
\mathrm{L}_{\mathrm{C}}=\mathrm{k}_{\alpha} \cdot \sigma_{0}
$$

where

$\mathrm{k}_{\alpha}=$ abscissa of the standardized normal distribution corresponding to the probability level $1-\alpha$. For $95 \% \mathrm{Cl}$ and one-tailed statistics, $\mathrm{k}_{\alpha}=1.65$;

$\sigma_{0}=$ standard deviation when the limiting mean of the net signal is zero;

The detection limit is based on the value determined for $\mathrm{L}_{C}$, the maximum allowable value for a Type II error $(\beta)$, and the standard deviation of the net signal when the limiting mean is equal to $\mathrm{L}_{\mathrm{D}}$. The detection limit equation is defined as:

$$
\mathrm{L}_{\mathrm{D}}=\mathrm{L}_{\mathrm{C}}+\mathrm{k}_{\mathrm{B}} \cdot \sigma_{\mathrm{D}}
$$

where

$\mathrm{k}_{\beta}=$ abscissa of the standardized normal distribution corresponding to the probability level $1-\beta$. For $95 \% \mathrm{CI}$ and one-tailed statistics, $\mathrm{k}_{\beta}=1.65$;

$\sigma_{\mathrm{D}}=$ standard deviation when the limiting mean of the net signal is $\mathrm{L}_{\mathrm{D}}$;

For the case of an active assay in CWAM where the background is much greater than the net signal, the variations in the standard deviations from a net signal of zero to $L_{D}$ is slight.

Therefore, the standard deviation of the net signal can be assumed constant, i.e. $\sigma_{D}=\sigma_{0}=\sigma$. For the variance of a net signal given by:

$$
\sigma^{2}=\sigma_{\mathrm{S}+\mathrm{B}}^{2}+\sigma_{\mathrm{B}}^{2}
$$

where

$$
\begin{aligned}
& \sigma_{S+B}^{2}=\text { variance of the "sample + blank" signal; } \\
& \sigma_{B}^{2}=\text { variance of the blank signal; }
\end{aligned}
$$




\begin{tabular}{|l|l|r|}
\hline & Canberra Industries, ACK\# D0043 & 33296 B \\
\hline Created: 29-September-2000 & IBIS Project & Revision B \\
\hline Revised: 13-January-2003 & Preliminary Design Study & Page 139 of 156 \\
\hline
\end{tabular}

this assumption results in the variance being equal to twice the blank variance or

$$
\sigma^{2}=2 \cdot \sigma_{B}^{2}
$$

If the standard deviation of the blank has been made negligible due to multiple observations as in the case of CWAM, the variance of the net signal is simply equal to the variance of the blank. For paired observations where the background is not known well, the standard deviation will differ from the case of the well known blank by the square root of two.

If the risk of $5 \%$ for a Type I or Type II error is acceptable ( $95 \%$ CI for one-tailed statistics), the following equations for the decision and detection limits are valid for active measurements in CWAM:

$$
\begin{aligned}
& \mathrm{L}_{\mathrm{C}}=1.64 \cdot \sigma_{\mathrm{B}} \\
& \mathrm{L}_{\mathrm{D}}=3.29 \cdot \sigma_{\mathrm{B}}
\end{aligned}
$$

Tables 20 and 21 show the decision and detection levels for the best, worse, and average positions for each surrogate matrix. A more useful parameter for Tennessee land fill regulations is the detection level in terms of activity concentration of matrix material. 


\begin{tabular}{|l|l|r|}
\hline & Canberra Industries, ACK\# D0043 & 33296 B \\
\hline Created: 29-September-2000 & IBIS Project & Revision B \\
\hline Revised: 13-January-2003 & Preliminary Design Study & Page 140 of 156 \\
\hline
\end{tabular}

Table 20. Decision levels in $\mathrm{mg}{ }^{235} \mathrm{U}$ for the best, worse, and average positions for $\alpha=\beta=0.05$ ( $95 \%$ confidence level).

\begin{tabular}{|l|c|c|c|}
\hline $\begin{array}{c}\text { Surrogate } \\
\text { Matrix }\end{array}$ & $\begin{array}{c}\text { Best Position } \\
\left(\mathbf{m g}{ }^{\mathbf{2 3 5}} \mathbf{U}\right)\end{array}$ & $\begin{array}{c}\text { Worse Position } \\
\left(\mathbf{m g}^{\mathbf{2 3 5}} \mathbf{U}\right)\end{array}$ & $\begin{array}{c}\text { Crate Average } \\
\left(\mathbf{m g}^{\mathbf{2 3 5}} \mathbf{U}\right)\end{array}$ \\
\hline Empty B-25 & - & - & 6.3 \\
\hline Empty SWB & - & - & 5.6 \\
\hline Dry Combustibles & 6.3 & 9.9 & 7.4 \\
\hline Polyethylene & 4.4 & 9.1 & 5.4 \\
\hline Scrap Metal & 13.3 & 39.8 & 16.1 \\
\hline Wet Combustibles & 9.7 & 24.6 & 13.8 \\
\hline
\end{tabular}

Table 21. Detection levels in $\mathrm{mg}{ }^{235} \mathrm{U}$ for the best, worse, and average positions for $\alpha=\beta=0.05$ (95\% confidence level).

\begin{tabular}{|l|c|c|c|}
\hline $\begin{array}{c}\text { Surrogate } \\
\text { Matrix }\end{array}$ & $\begin{array}{c}\text { Best Position } \\
\left(\mathbf{m g}{ }^{\mathbf{2 3 5}} \mathbf{U}\right)\end{array}$ & $\begin{array}{c}\text { Worse Position } \\
\left(\mathbf{m g ~}^{\mathbf{2 3 5}} \mathbf{U}\right)\end{array}$ & $\begin{array}{c}\text { Crate Average } \\
\left(\mathbf{m g}^{\mathbf{2 3 5}} \mathbf{U}\right)\end{array}$ \\
\hline Empty B-25 & - & - & 12.5 \\
\hline Empty SWB & - & - & 11.2 \\
\hline Dry Combustibles & 12.7 & 19.9 & 14.9 \\
\hline Polyethylene & 8.8 & 18.2 & 10.9 \\
\hline Scrap Metal & 26.6 & 79.7 & 32.3 \\
\hline Wet Combustibles & 19.4 & 49.4 & 27.7 \\
\hline
\end{tabular}

Tables 22 and 23 show the detection limits in terms of specific activity for depleted $\left(0.2 \%{ }^{235} \mathrm{U}\right)$ and highly enriched $\left(93.3 \%{ }^{235} \mathrm{U}\right)$ form, respectively. Activity calculations include contributions from ${ }^{234} \mathrm{U},{ }^{235} \mathrm{U},{ }^{236} \mathrm{U}$, and ${ }^{238} \mathrm{U}$. 


\begin{tabular}{|l|l|r|}
\hline & Canberra Industries, ACK\# D0043 & 33296 B \\
\hline Created: 29-September-2000 & IBIS Project & Revision B \\
\hline Revised: 13-January-2003 & Preliminary Design Study & Page 141 of 156 \\
\hline
\end{tabular}

Table 22. Detection levels in $\mathrm{pCi} / \mathrm{g}$ for depleted uranium for the best, worse, and average positions for $\alpha=\beta=0.05$ ( $95 \%$ confidence level).

\begin{tabular}{|l|c|c|c|}
\hline $\begin{array}{c}\text { Surrogate } \\
\text { Matrix }\end{array}$ & $\begin{array}{c}\text { Best Position } \\
(\mathbf{p C i} / \mathbf{g})\end{array}$ & $\begin{array}{c}\text { Worse Position } \\
\text { (pCi/g) }\end{array}$ & $\begin{array}{c}\text { Crate Average } \\
\text { (pCi/g) }\end{array}$ \\
\hline Dry Combustibles & 8.3 & 13.0 & 9.7 \\
\hline Polyethylene & 5.4 & 11.1 & 6.6 \\
\hline Scrap Metal & 5.9 & 17.6 & 7.1 \\
\hline Wet Combustibles & 6.9 & 17.7 & 9.9 \\
\hline
\end{tabular}

Table 23. Detection levels in $\mathrm{pCi} / \mathrm{g}$ for $93.3 \%$ enriched uranium for the best, worse, and average positions for $\alpha=\beta=0.05$ (95\% confidence level).

\begin{tabular}{|l|c|c|c|}
\hline \multicolumn{1}{|c|}{$\begin{array}{c}\text { Surrogate } \\
\text { Matrix }\end{array}$} & $\begin{array}{c}\text { Best Position } \\
(\mathbf{p C i} / \mathbf{g})\end{array}$ & $\begin{array}{c}\text { Worse Position } \\
(\mathbf{p C i} / \mathbf{g})\end{array}$ & $\begin{array}{c}\text { Crate Average } \\
(\mathbf{p C i} / \mathbf{g})\end{array}$ \\
\hline Dry Combustibles & 3.0 & 4.8 & 3.6 \\
\hline Polyethylene & 1.9 & 4.1 & 2.4 \\
\hline Scrap Metal & 2.1 & 6.4 & 2.6 \\
\hline Wet Combustibles & 2.5 & 6.5 & 3.6 \\
\hline
\end{tabular}

From the results in Tables 21 and 22, it is clear that CWAM is able to screen both depleted and enriched uranium at the $35 \mathrm{pCi} / \mathrm{g}$ level at all positions within all the surrogate matrices. 


\begin{tabular}{|l|l|r|}
\hline & Canberra Industries, ACK\# D0043 & 33296 B \\
\hline Created: 29-September-2000 & IBIS Project & Revision B \\
\hline Revised: 13-January-2003 & Preliminary Design Study & Page 142 of 156 \\
\hline
\end{tabular}

\section{New Work}

In order to bring the central themes of this study to a wider audience for critical review results have been presented at several meetings. The reader is directed to the following publications for details.

\section{PUBLICATIONS}

1. S Croft, R McElroy, D Martancik, B Young, D B Syme, P M J Chard, R J Estep and S Melton 'IBIS' - the Integrated Box Interrogation System

7th Nondestructive Assay Waste Characterization Conference, 23-25 May 2000, Salt Lake City, Utah

Idaho National Engineering and Environmental Laboratory Report INEEL/EXT-20000002, Vol 1 (2000) 103-121.

2. S Croft, B M Young, D Martancik, J R Wachter, P M J Chard, R J Estep and S Melton

The Box Segmented Neutron Scanner Component of the Proposed Integrated Box Interrogation System, IBIS

Proceedings of $42^{\text {nd }}$ Annual Meeting of the INMM (Institute of Nuclear Materials Management), 15-19 July 2001, Indian Wells, California, USA. CD-ROM @ 2001. Session H, Paper 271, Tuesday July 17

3. B M Young, S Croft, D Martancik, R J Estep and S Melton

Conceptual Design of a Box Segmented Gamma Scanner for the Proposed Integrated Box Interrogation System, IBIS

Proceedings of $42^{\text {nd }}$ Annual Meeting of the INMM (Institute of Nuclear Materials

Management), 15-19 July 2001, Indian Wells, California, USA. CD-ROM (c) 2001.

Session G, Paper 273, Tuesday July 17

4. $2002 \mathrm{NMM}$

S Croft, L C-A Bourva, P M J Chard, S Melton

Principles of Fast Neutron Detector Package Design for Differential Die-away Technique Assay Systems Proc. 43 ${ }^{\text {rd }}$ Annual Meeting of the INMM, June 23-27, 2002, Florida, USA. To be published. 


\begin{tabular}{|l|l|r|}
\hline & Canberra Industries, ACK\# D0043 & 33296 B \\
\hline Created: 29-September-2000 & IBIS Project & Revision B \\
\hline Revised: 13-January-2003 & Preliminary Design Study & Page 143 of 156 \\
\hline
\end{tabular}

In addition, two further papers of particular relevance have recently become available. These are also commented to the interested reader.

\title{
NEUTRON COINCIDENCE IMAGING FOR ACTIVE
} AND PASSIVE NEUTRON ASSAYS

Robert J. Estep, Glen Brunson, and Sheila Melton

Los Alamos National Laboratory

Los Alamos, New Mexico, 87545, USA 505/667-3683

\section{SENSITIVITY AND ACCURACY CONSIDERATIONS FOR NEUTRON ASSAY OF PLUTONIUM-CONTAMINATED WASTE IN LARGE CONTAINERS}

\author{
Sheila G. Melton, Robert J. Estep \\ Los Alamos National Laboratory \\ Los Alamos, NM 87545
}

Proc $42^{\text {nd }}$ Annual Meeting of the INMM, 2001 


\begin{tabular}{|l|l|r|}
\hline & Canberra Industries, ACK\# D0043 & 33296 B \\
\hline Created: 29-September-2000 & IBIS Project & Revision B \\
\hline Revised: 13-January-2003 & Preliminary Design Study & Page 144 of 156 \\
\hline
\end{tabular}

\section{References and Bibliography}

1. Abdurrahman, N.M., Lee, Y.D., Block, R.C., and Slovacek, R.E.

Neutron Emission Tomography for Nuclear Fissile Materials Safeguards

Proceedings of the $5^{\text {th }}$ International Conference on Facility Operations Safeguards Interface Jackson Hole, USA, 1995, pp 18 - 19.

2. Armitage, B.H.

Detector Package Assessment for a 2081 Barrel Dieaway chamber, Private Communication, Doc. Ref. PWMWG/P79.

3. Armitage, B.H.

Lead Slowing Down Spectrometer: Initial Examination of Applicability to the Quality Checking of Intermediate and High Level Waste. AERER13048, Feb 1989.

4. Armitage, B.H. and Cogbill, M.J.

Dual Active Neutron Interrogation of Fissile Material in Waste Drums

Proceedings of the $11^{\text {th }}$ ESARDA Symposium on Safeguards and Nuclear Materials

Management,

Luxembourg, 30 May - 1 June 1989, pp 515 - 518, EUR-12193-EN.

5. Armitage, B.H. and Cogbill, M.J.

Dual Active Neutron Interrogation of Fissile Material in Waste Drums.

Proceedings of the 11th ESARDA Symposium on Safeguards and Nuclear Material

Management, Luxembourg, 30 May - 1 June 1989, pp 515 -518. EUR 12193 EN.

6. Armitage, B.H. and A.C. Sherwood

Matrix Effects in the Neutron Die-Away Assay of Fissile Material in 2001 drums

Proceedings of the 9th ESARDA Symposium on Safeguards and Nuclear Material

Management, London, 12 - 14 May 1987, pp 401 - 410.

7. Armitage, B.H., Chard, P.M.J., Croft S., Lambert K.P., and Lloyd D.J.

A Scan a Source Matrix Correction Technique for Use with a Reconfigurable Passive

Neutron Plutonium Assay System, .Nuclear Instruments and Methods Research A 354, 1995, pp 52-526.

8. Arnone, G.J

PATRM/PCI design specification

Rev 1.028 June (2000)

Los Alamos National Laboratory report prepared under US DOE contract W-7405-ENG-36. 


\begin{tabular}{|l|l|r|}
\hline & Canberra Industries, ACK\# D0043 & 33296 B \\
\hline Created: 29-September-2000 & IBIS Project & Revision B \\
\hline Revised: 13-January-2003 & Preliminary Design Study & Page 145 of 156 \\
\hline
\end{tabular}

9. Arnone, G.J., Brunson, G.S., Coop, K.L.,

"A Pulse Arrival Time Recording Module for Analyzing Neutron Multiplicities", IEEE Nuclear Science Symposium, Orlando, Florida, 27-31 October 1992. Also Los Alamos National Laboratory report LA-UR-92-3460, 1992.

10. Becker, G.K., Mcllwain, M., and Connolly, M.

"Transuranic and Low-Level Boxed Waste Form Nondestructive Assay Technology Overview and Assessment", Idaho National Engineering and Environmental Laboratory Publication INEEL/EXT-99-00121, February 1999. Idaho National Engineering and Environmental Laboratory

11. Becker, G. K., Roney, T.J., Watts, C.L., and Bennion, J.S. Utility of Neural Networks in Nondestructive Waste Assay Measurement Methods Proceedings of the $4^{\text {th }}$ Nondestructive Assay and Nondestructive Examination Waste Characterization Conference, Salt Lake City, Utah, 24 - 26 October 1995, pp 161 - 187.

12. Bell, G.I. and Glasstone, S.

Nuclear Reactor Theory Van Nostrand Reinhold Company (N.Y., 1970)

13. Bergman, A.A., Isacoff, A. I., Murin, I.D., Shapiro, F.L., Shtranikh, . I.V., and Cazarnovsky, M.V.

A Neutron Spectrometer Based on Measuring the Slowing-Down Time of Neutrons in Lead, Peaceful Uses of Atomic Energy 4, 1955, pp 135 - 146.

14. Blackwood, L.G. , Harker, Y.D., and Meachum, T.R. Passive Active Neutron Radioassay Measurement Uncertainty for Aqueous Sludge Waste JNMM, Spring, 1999, pp $33-46$.

15. Block, R.C., Slovacek, R.E., Hoff ; R.W., and Nakagome, Y.

Technique for Fission Measurement of Highly Active and Weak Cross Section Actinides 50 Years with Nuclear Fission Vol. 1

J.W. Behrens and A.D. Carlson (Eds)

Publ. American Nuclear Society (USA, 1989) 354- 359

16. Briemeister, J.F., (Ed). "MCNPTM - A General Monte-Carlo Code for Neutron and Photon Transport". Los Alamos Laboratory Report LA-12625-M. Version 4B (March 1997)

17. Bronson F.C. "Q $\mathrm{Q}$ - A Very Low Quantitative and Qualitative Waste Assay and Release Certification System" Waste Management 1994, Tucson, Arizona, February 1990. 


\begin{tabular}{|l|l|r|}
\hline & Canberra Industries, ACK\# D0043 & 33296 B \\
\hline Created: 29-September-2000 & IBIS Project & Revision B \\
\hline Revised: 13-January-2003 & Preliminary Design Study & Page 146 of 156 \\
\hline
\end{tabular}

18. Bronson F. C. and Young B.M.

"Mathematical Calibration of Ge Detectors, and the Instruments That Use Them"

NDA/NDE Waste Characterization Conference, 1997

19. Bronson F.C., Young B.M., and Atraskevich V.

"ISOCS Mathematical Calibration Software for Germanium Gamma Spectroscopy Small and Large Objects", Proceedings of the 1997 American Nuclear Society Annual Meeting,

Orlando, Florida, 1-5 June 1997.

20. Bruggeman, M, Baeten, P., DeBoeck, W., and Carchon, R.

Neutron Coincidence Counting Based on Time Internal Analysis with One- and Two-

Dimensional Rossi-Alpha Distributions: An Application for Passive Neutron Waste Assay

Nuclear Instruments and Methods in Physics Research A382, 1996, pp511 - 518.

21. Brunson, G.S.

An Extension to the Feynman Method of Detecting Fission Neutrons

Nuclear Instruments and Methods 214, 1983, pp $341-348$.

22. Brunson, G.S., and Arnone, G.J.

Applications of a Versatile New Instrument Module

Proceedings of the 5th Nondestructive Assay and Nondestructive Examination Waste

Characterization Conference, Salt Lake City, Utah, 14-16 January 1997, pp 153-173.

Conf-970126

23. Brunson, G.S., and Nicholas, N.J.

"Shift-Register Neutron-Coincidence Counting and the Gray Barrel Problem,"

Los Alamos Report, October 1992, LA-12414-MS.

24. Caldwell, J. T., Hastings, R. D., Herrera, G. C., and Kunz, W. E., et al.

"The Los Alamos Second-Generation System for Passive and Active Neutron Assays of

Drum Size Containers," Los Alamos National Laboratory Report LA-10774-MS, September 1986.

25. Caldwell, J.T., Bieri, J.M., Embry, W.N., Kuckertz, T.H., Newell, M.R., Plettenberg, K.B., Peterson, T.A,. and West, R.E.

High Sensitivity Imaging Passive and Active Neutron (IPAN) Transuranic Assay System Proceedings of the $14^{\text {th }}$ Annual European Safeguards Research and Development Association (ESARDA) Meeting, Salamanca, Spain, 5 - 8 May, 1992, pp 257 -261. ESARDA 25

26. Canberra Industries, A Division of Packard BioSciences, Inc., 800 Research Parkway, Meridan Connecticut. 


\begin{tabular}{|l|l|r|}
\hline & Canberra Industries, ACK\# D0043 & 33296 B \\
\hline Created: 29-September-2000 & IBIS Project & Revision B \\
\hline Revised: 13-January-2003 & Preliminary Design Study & Page 147 of 156 \\
\hline
\end{tabular}

27. Chard, P.M.J.

Development of Active Neutron Interrogation Techniques for the Assay of Fissile Material in Drummed Waste. Ph.D. thesis, University of Birmingham, 1991.

28. Chard, P.M.J.

Initial Investigations of a Performance of a Modified DDA System at sub-mb levels, Private Communication, 5 November 1992.

29. Chard, P.M.J. and Croft S.

Self Shielding Factors for the Cf-shuffler Neutron Interrogation Technique Proceedings of the 17th ESARDA Symposium on Safeguards and Nuclear Material Management, Aachen, Germany, 9-11 May 1995, pp 189 -194. EUR 16290 EN.

30. Chard, P.M.J. and Croft S.

Self Shielding Factors for the Cf-Shuffler Neutron Interrogation Technique Proceedings of the 17th ESARDA Symposium on Safeguards and Nuclear Material Management, Aachen, Germany, 9-11 May 1995, pp 557-562. EUR 16290 EN.

31. Chard, P.M.J. and Croft, S.

A Database of ${ }^{240} \mathrm{Pu}_{\mathrm{effective}}$ and ${ }^{235} \mathrm{U}_{\text {effective }}$ Coefficients for Various Fertile and Fissile Isotopes. Proceedings of the 19th ESARDA Symposium on Safeguards and Nuclear Material Management, Montpellier, France, 13-15 May 1997, pp 389 - 396. EUR 17665 EN.

32. Chard, P.M.J. and Croft, S.

Preliminary Investigation of Active Neutron Coincidence Counting in Differential Die-Away Assay, Proceedings of the $7^{\text {th }}$ International Conference on Radioactive Waste Management and Environmental Remediation, Nagoya, Japan, 26 - 30 September 1999, ICEM '99 CD ROM ISBN 0-7918-1966-3, Session 32, Paper 3.

33. Chard, P.M.J, Croft, S., and Sharp, P.B. Accurate Matrix Compensation for Fissile Material Assay in Drummed Waste Using a High Sensitivity Differential Die-away Chamber, Proceedings $7^{\text {th }}$ Nondestructive Assay Waste Characterization Conference, Salt Lake City, Utah, 22-26 May 2000. 


\begin{tabular}{|l|l|r|}
\hline & Canberra Industries, ACK\# D0043 & 33296 B \\
\hline Created: 29-September-2000 & IBIS Project & Revision B \\
\hline Revised: 13-January-2003 & Preliminary Design Study & Page 148 of 156 \\
\hline
\end{tabular}

34. Computer I/O Corporation

World Wide Web site 'computerio.com' (2000).

35. Cogbill, M.J. and Swinhoe, MT

Self Shielding Factors for Neutron Energies up to $0.75 \mathrm{keV}$

United Kingdom Atomic Energy Authority Safeguards R\&D Project report SRDP -

R126(1985).

36. Coop, K.L.

A Combined Thermal/Epithermal Neutron Interrogation Device to Assay Fissile Materials in Large Containers

Proceedings of the $11^{\text {th }}$ ESARDA Symposium on Safeguards and Nuclear Material

Management, Luxembourg, 30 May - 1 June 1989, pp 201 - 206, EUR-12193-EN.

37. Coop, K.L.

Neutron Die-Away Methods for Criticality Safety Measurements of Fissile Waste

Las Alamos National Laboratory report LA-UR-89-2104

also: Nuclear Criticality Safety Division of the American Nuclear Society International

Topical Meeting on Safety Margins in Criticality Safety, San Francisco, California,

November 26-30,1989

38. Coop, K.L. and Hollas, C.L.

Epithermal Neutron Interrogation of Fissile Waste

Proceedings of the $37^{\text {th }}$ Annual Meeting of the Institute of Nuclear Materials Management, 1996 , pp $1263-1268$.

39. Crane, T. W.

"Detectability Limits and Precision for Shufflers," Los Alamos National Laboratory report LA-10158-MS, August 1984.

40. Croft, S., R. McElroy,D. Martancik,B. young, DB Syme, P.M.J. Chard, R.J. Estep and S. Melton

"IBIS - The Integrated Box Interrogation System",

Proceedings of the $7^{\text {th }}$ Nondestructive Assay Waste Characterization Conference

Salt Lake City, Utah, 23 - 25 May 2000.

Idaho National Engineering and Environmental Laboratory Report INEEL/EXT-2000-

0002)2000) 103-121.

41. Currie

L.A."Limits for Qualitative and Quantitative Determination", Analytical Chemistry Volume 40, pp 586-593, March 1968.

42. Czubek, J.A.

Pulsed Neutron Method for Uranium Well Logging

Geophysics 37, 1972, pp 160-173. 


$\mid$\begin{tabular}{|l|l|r|}
\hline & Canberra Industries, ACK\# D0043 & 33296 B \\
\hline Created: $29-S e p t e m b e r-2000$ & IBIS Project & Revision B \\
\hline Revised: 13-January-2003 & Preliminary Design Study & Page 149 of 156 \\
\hline
\end{tabular}

43. DOE/EM-0465 (Dec, 1998)

Innovative Technology Summary Report Prepared for the U.S. Department of Energy, Office of Environmental Management, Office of Science and Technology.

Nondestructive Waste Assay Using Combined Thermal Epithermal Neutron Interrogation

44. Drozdowicz, K., Igielski, A., Krynnicka-Drozdowicz, E., Soczynski, Z. and Woznicka, U. Thermal Neutron Diffusion Parameters for Plexiglas.

Nuclear Instruments and Methods 178, 1980, pp 513-516.

45. East, L.V. and Becker, G.K.

Experience Gained From Passive/Active Neutron (PAN) Assay Measurements on 12,600

TRU waste drums at the INEL

Proceedings of the Nondestructive Assay and Nondestructive Examination Waste

Characterization Conference, Pocatello, Idaho, 14-16 February 1994, pp 143-166

CONF - 940216

46. ENDF/B-6 . web site: http//t2.lanl.gov/data/map.html.

JEF - 2.2 . CD-ROM distributed by the OECD NEA Data Bank.

47. Estep, R.J.

"TGS_FIT 3.1 User's Manual" to be published.

48. Estep, R.J., Buenafe,C, and Melton, S.

"The CTEN_FIT User's Manual", Los Alamos Report NIS6-QAP-0018 (in revision), April, 2000.

49. Estep, R.J., Buenafe, C, Melton, S., and Miko D.

Tomographic Assay Techniques in the Combined Thermal/Epithermal Neutron (CTEN)

Method, Proceedings of the $40^{\text {th }}$ Annual Institute of Nuclear Materials Management Meeting, Phoenix, Arizona, 25-29 July 1999.

LLC, CD_ROM (1999)

50. Estep, R.J., and Cavender, J.

"The WIN_TGS Software Package for Tomographic Gamma Scanner Systems", Proceedings of the 35th INMM Meeting, Naples, FL, 17-20 July 1994.

51. Estep, R.J., Coop, K. L., Hollas, C., Melton, S, and Miko, D.

"Lump Correction and Identification in the Combined Thermal/Epithermal Neutron (CTEN) Method", Proceedings of the INMM 39th Annual Meeting, Naples Florida, 26-30 July 1998.

52. Estep, R.J., S. Melton and D. Miko

Methods for Reducing Bias in Combined Thermal/Epithermal Neutron (CTEN) Assays of Heterogeneous Waste 


\begin{tabular}{|l|l|r|}
\hline & Canberra Industries, ACK\# D0043 & 33296 B \\
\hline Created: 29-September-2000 & IBIS Project & Revision B \\
\hline Revised: 13-January-2003 & Preliminary Design Study & Page 150 of 156 \\
\hline
\end{tabular}

Proceedings of the $6^{\text {th }}$ Nondestructive Assay Waste Characterization Conference

Salt Lake City, Utah, 17 - 19 November 1998, pp 515 - 528. CONF-981105.

53. Estep, R.J., et al.

"User's Manual for WIN_CTEN 1.0", NIS6-QAP-0024 (in revision), April, 2000.

54. Giesler G.C., Henry S.A., JohnsonS.L., and Vehar D.W.

"Calibration and Characterization of a Low Level Waste Assay System", Waste Management 1994, Tucson, Arizona, February 1994, pp 2175-2179.

55. Glasstone, S. and Edlund, M.C.

"The Elements of Nuclear Reactor Theory", 6th printing.

D. Van Nostrand Company Inc. (N.Y., 1957).

56. Granada, J.R., Dawidowski, J., Mayer, R.E,. and Gillette, V.H.

Thermal Neutron Cross Section and Transport Properties of Polyethylene.

Nuclear Instruments and Methods in Physics Research A261 (1987) 573-578.

57. Guide Technology Incorporated

1630 Zanker Road, San Jose, CA 95112, USA

Application notes and technical literature. Ron Sigura Priv. Comm. 1998

58. Gunink, R.

"MGA: A Gamma-Ray Spectrum Analysis Code for Determining Plutonium Isotopic Abundances", UCRL-LR-103220, Vol. I (ISPO No. 317, Task No. A.161), April 3, 1990.

59. Gunink, R. and Ruhter, W.D.,

"MGA: A Gamma-ray Spectrum Analysis Code for Determining Plutonium Isotopic

Abundances", UCRL-LR-103220, Vol. 2 (ISPO No. 317, Task No. A.161), September 1, 1990. 


\begin{tabular}{|l|l|r|}
\hline & Canberra Industries, ACK\# D0043 & 33296 B \\
\hline Created: 29-September-2000 & IBIS Project & Revision B \\
\hline Revised: 13-January-2003 & Preliminary Design Study & Page 151 of 156 \\
\hline
\end{tabular}

60. Gunink, R., Ruhter, W.D., Miller, P., Goerten, J., Swinhoe, M.T., Wagner, H.G., Verplancke, J.M.A. Bickel M., and Abousahl, S.

"MGAU: A New Analysis Code for Measuring Uranium-235 Enrichments in Arbitrary Samples", Proceedings of the International Safeguards Symposium, Vienna, IAEA-SM333/88P, March 1994, pp 541-546

61. Hampson, H.F.

BNFL THORP Plant Radiometric Instrumentation Nuclear Energy 32, 1993, pp $15-22$.

62. Harker, Y.D., Blackwood, L.G. and Meachum, T.R. Uncertainty Analysis of the SWEPP Drum Assay System for Graphite Content Code 300 Proceedings of the $4^{\text {th }}$ Nondestructive Assay and Nondestructive Examination Waste Characterization Conference, Salt Lake City, Utah, 24 - 26 October 1995, pp 189-239. CONF-951091

63. J. Verplancke, D.Davidson, B. Gillespie, M. Koskelo, F. Bronson, R. Gunink, J.-L. Ma, J. Romeyer-Dherbey, S. Aboushal and M. Bickel, "Applying MGA for Waste Characterization", Presented at the WM95 conference, Feb26-Mar 2, 1995 Tucson, AZ.

64. Haruyama, M., Sugimoto, Y., Takase, M., Osugi . T. and Ara, K. R\&D of New Detection Method for Trace Amount of Fissionable Material in Waste Research Group for Reactor Physics, Department of Nuclear Energy System, Japan Atomic energy Research Institute, Tokai-mura, Naka-gun, Ibaraki-Ken 319-11, Japan. Note dated 6 Sept. 1999

65. Hollas, C. L., et al., "Matrix Effects Corrections in DDT Assays of ${ }^{239} \mathrm{Pu}$ with the CTEN Instrument," 5th Annual NDA and NDE Waste Characterization Conference, Salt Lake City, January, 1997.

66. Hollas, C. L, Arnone, G.J., Brunson G.S., and Coop, K.L.

Determination of fission neutron transmission through waste matrix material using neutron signal correlation from active assay of $239 \mathrm{Pu}$ Proceedings of the 37th Annual Meeting Institute of Nuclear Materials Management, Naples, Florida, 28 Jul - 1 Aug 1996, pp 1269 - 1274 Los Alamos National Laboratory report LA-UR-96-2574

67. Hollas, C. L, Arnone, G.J., Brunson G.S., Coop K.L., and Melton, S.G. Matrix effects corrections in DDT assay of 239Pu with the CTEN instrument Proceedings of the 5th Nondestructive Assay and Nondestructive Examination Waste Characterization Conference, Salt Lake City, Utah, 14-16 January 1997, pp 175 - 182 Conf-970126 


\begin{tabular}{|l|l|r|}
\hline & Canberra Industries, ACK\# D0043 & 33296 B \\
\hline Created: 29-September-2000 & IBIS Project & Revision B \\
\hline Revised: 13-January-2003 & Preliminary Design Study & Page 152 of 156 \\
\hline
\end{tabular}

68. Koppel, J

"Time Dependent Neutron Spectra", Nuclear Science and Engineering, Volume 8, pp 157$163,1960$.

69. H. Krinninger and E. Ruppert

Operational Experience with the Automatic Lead Spectrometer Facility for Nuclear

Safeguards, Trans. American Nuclear Society 15, 1972, pp 683 - 684.

70. Krinninger, H., Wiesner S., and Faber, C.

Pulsed Neutron Method for Nondestructive and Simultaneous Determination of the ${ }^{235} \mathrm{U}$ and

${ }^{239} \mathrm{Pu}$ Contents of Irradiated and Non-irradiated Reactor Fuel Elements

Nuclear Instruments and Methods. 73, 1969, pp $13-33$.

71. Kunz, W. E., Atencio, J. D. and Caldwell, J. T.

"A 1-nCi/g Sensitivity Transuranic Waste Assay System Using Pulsed Neutron Interrogation", $21^{\text {st }}$ Annual Meeting Proceedings of the Nuclear Materials Management, Volume 11, July 1980.

72. Kunz, W. E., Atencio, J. D. Bernard, W., Herrera, G.C., Pratt, J.C. and Caldwell, J.T.

"A 1-mg-Sensitivity Fissile Assay System"

Proceedings of the $3^{\text {rd }}$ ESARDA Symposium on Safeguards and Nuclear Materials

Management, Karlsruhe, F.R. Germany 6-8 May 1981, pp $119-122$

ESARDA Report 13

73. Lestone, J.P.

Subroutines to Simulate Fission Neutrons for Monte Carlo Transport Codes

Los Alamos National Laboratory Report LA-UR-99-5444, 1999.

74. Lillie, R.A., Santoro, R.T., and Alsmiller, Jr., R.G.

Calculation of Neutron Die-Away Times in a Large-Vehicle Portal Monitor

Nuclear Technology 52, 1981, pp 313 - 318.

75. Littler, D.J. and Raffle, J.F.

An Introduction to Reactor Physics. 2nd Ed., Pergamon Press (London, 1957)

76. Lucero R.F., and Caldwell, J.T.

Measurement Results for Two Automated IPAN Drum Assay Systems

Proceedings of the $37^{\text {th }}$ Annual Institute of Nuclear Materials Management Meeting, 28 July

$-1^{\text {st }}$ Aug 1996, Naples, Florida, USA pp 1304-1310

77. Ma, J.L., Mariani, A., Martin-Deidier, L., Pasquali , R. , and Romeyer-Dherbey, J.

Development of advanced device for low level waste assay based on $14 \mathrm{MeV}$ neutron interrogation

Proceedings of the $35^{\text {th }}$ INMM Annual Meeting , 17 - 20 July 1994, Naples, Florida, USA 


\begin{tabular}{|l|l|r|}
\hline & Canberra Industries, ACK\# D0043 & 33296 B \\
\hline Created: 29-September-2000 & IBIS Project & Revision B \\
\hline Revised: 13-January-2003 & Preliminary Design Study & Page 153 of 156 \\
\hline
\end{tabular}

78. Melton, S.G.

"CWAM Created Waste Assay Monitor - System Redesign Package", April 15, 1996

79. Melton, S.G., Estep, R.J., and Hollas, C.L.

Implementation of Advanced Matrix Corrections for Active Interrogation of Waste Drums

Using the CTEN Instrument

Los Alamos National Laboratory report LA-UR-98-3250

Presented at the INMM Annual Meeting, 26 - 30 July 1998.

80. Melton, S.G., Estep, R.J., and Hollas, C.L.,

"Monte Carlo Simulation of Active and Passive Measurements in the CTEN Instrument", Proceedings of the $40^{\text {th }}$ Ann. INMM Meeting, Phoenix, Arizona, 1999.

81. Melton, S.G., Estep, R.J., Hollas, C.L., Arnone, G.J., Brunson, G.S., and Coop, K.L.

"Development of Advanced Matrix Correction Techniques for Active Interrogation of Waste Drums Using the CTEN Instrument", Proceedings INMM 38th Annual Meeting, Phoenix, Arizona, 20-24 July 1997.

82. Melton, S.G., Estep, R.J., and Peterson, E.

Calibration of the Crated Waste Assay Monitor for the Low-Level Waste Measurements for the Y-12 Plant, Proceedings of the $7^{\text {th }}$ Nondestructive Assay Waste Characterization Conference, Salt Lake City, Utah, 23 - 25 May 2000

Idaho National Engineering and Environmental Laboratory Report INEEL/EXT-2000-0002. (2000) 87-102.

83. Melton, S.G., Estep, R.J., and Peterson, E.

"The Differential Die-Away Technique (DDT) as Implemented in the Crated Waste Assay

Monitor (CWAM), Private Communication, 9 November 1999.

84. Nicholas, N. J., Coop, K. L., and Estep, R. J.

"Capabilities and Limitations of the Passive-Active DDT Neutron Waste Assay Device",

$33^{\text {rd }}$ Annual Meeting Proceedings of the Nuclear Material Management, July 1992, Volume 21, pp 200-210.

85. "National Transuranic Program Quality Assurance Program Plan", Section 9.0. Revision Interim Change, November 11, 1996.

86. Packer, T.W. and Swinhoe, M.T.

The Differential Die-Away Technique Applied to the Measurement of the Fissile Content of Drums of Cement Encapsulated Waste

UKAEA Harwell Laboratory Report AERE-R-13137, 1988.

87. Pál, L.I.

Statistical fluctuations of neutron multiplication

Proceedings of the $2^{\text {nd }}$ United Nations International Conference on the Peaceful Uses of 


\begin{tabular}{|l|l|r|}
\hline & Canberra Industries, ACK\# D0043 & 33296 B \\
\hline Created: 29-September-2000 & IBIS Project & Revision B \\
\hline Revised: 13-January-2003 & Preliminary Design Study & Page 154 of 156 \\
\hline
\end{tabular}

Atomic Energy, Geneva, 1 - 13 Sept 1958, Volume 16, pp 687 - 696.

Nuclear Data and Reactor Theory. United Nations, Geneva

88. Pázit, I. and Yamane, Y.

Theory of Neutron Fluctuations in Source-Drive Subcritical Systems

Nuclear Instruments and Methods. in Physics Research A403, 1998, pp 431 - 441.

89. Phillips A.

The PFE32 software was written by Alan Phillips, Lancaster University Computer Center.

Copies can be obtained from the web site $w w w$. winsite.com or www.simtel.net/simtel.net.

90. Reilly, D., Ensslin, N., Smith Jr., H., and Kreiner S.(eds)

Passive Nondestructive Assay of Nuclear Materials

NUREG/CR-5550 ISBN 0-16-032724-5 (1991)

Also Los Alamos National Laboratory report LA-UR-90-732, Chap 16, Principles of neutron coincidence counting, pp $457-491$, N. Ensslin.

91. Rinard, P.M., Coop, K.L., Nicholas, N.J. and Menlove, H.O.

Comparison of Shuffler and Differential Die-Away Technique Instruments for the Assay of Fissile Materials in 55-Gallon Waste Drums

NMM, July 1994, pp $28-38$.

92. Rinard P. M, Menlove, H. O., and et.al.

"The Nondestructive Assay of 55-gallon Drums Containing Uranium and Transuranic Waste Using Passive-Active Shuffler", Los Alamos National Laboratory report LA-12446-MS, November 1992.

93. Rotter, W.

Determination of Burn-up of Irradiated Fuel Elements by Pulsed Neutron Interrogation Annals of Nuclear Science and Engineering, 1974, pp $451-458$.

94. SAND77-0300 (Feb., 1977)

Prompt Neutron Uranium Borehole Logging with Prompt Fission Neutrons (PFN)

Neutron Tube Technology for PFN Logging Equipment

Proceedings of a seminar held Feb. 24, 1977, Airport Marina Hotel,

Albuquerque, New Mexico

Sandia National Laboratory, Albuquerque, N.M., Report

95. Sawan M.E. and Conn, R.W.

Neutron Pulses Slowing Down in Heavy Media Analysis with Applications of the Lead

Spectrometer, Nuclear Science and Engineering 54, 1974, pp 127 - 142.

96. Sawan M.E., Nagy M.E., and Bazan A.A. 


\begin{tabular}{|l|l|r|}
\hline & Canberra Industries, ACK\# D0043 & 33296 B \\
\hline Created: 29-September-2000 & IBIS Project & Revision B \\
\hline Revised: 13-January-2003 & Preliminary Design Study & Page 155 of 156 \\
\hline
\end{tabular}

Nondestructive Assay of Fuel Rods with Any Number of Fissile Isotopes Using the Lead Spectrometer

Atomkernenergie 39, 1981, pp 48 - 52.

97. Schrack R.A., Behrens J.W., Bowman C.D., and Carlson A.D.

Safeguards Reference Measurement System Utilizing Resonance Neutron Radiography

Measurement Technology for Safeguards and Material Control

T.R. Canada and B.S. Carpenter (Eds)

NBS Spec. Publ. 582, 1980, pp 86-92.

98. Smith, G.W. and Rice III, L.G.

Application of a $14 \mathrm{MeV}$ neutron source to the detection of special nuclear material diversion IEEE Trans on Nuclear Science NS-28, 1981, pp 1637 - 1639.

99. Solicitation DE-PS07-991D12812 - Boxed Waste Form NDA Development.

100. "Standardized Statistical Techniques for Radiological Measurements", Martin Marietta Energy Systems, Inc., position paper, September 1993.

101. Stegemann D.

"Application of Neutron Techniques in the Nondestructive Assay of Fissile Materials" Nuclear Technology, 13 April 1972, pp 59-64.

102. Stegemann, D. and Seufert, $H$.

Application of the Slowing Down Time Spectrometer for the Control of Fissionable Material, Trans. American Nuclear Society 11, 1968, pp 658-659.

103. Swinhoe, M.T.

The Differential Die-Away Technique Applied to the Measurement of the Fissile Content of Drums of Cement Encapsulated Waste UKAEA Harwell Laboratory Report AERE-R-12074 , 1986.

104. Tench, O.

Canberra Introduces the Broad Energy Germanium Detector, Canberra Chronicle, December 1997 , pp 5.

105. Vanterpool E.C., Slovacek R.E., Harris D.R., and Block R.C.

Fuel Assembly Assay be Neutron Interrogation in a Lead Slowing-Down-Time Spectrometer Nuclear Science and Engineering 110, 1992, pp 186- 194.

106. "Waste Acceptance Criteria for the Waste Isolation Pilot Plant, Revision 5" DOE/WIPP-069.

107. Young B.M, Gunink, R. and Ruhter, W.D.

Distributed by Canberra Industries, a Division of Packard BioScience , 800 Research Parkway, Meriden, Connecticut. 


\begin{tabular}{|l|l|r|}
\hline & Canberra Industries, ACK\# D0043 & 33296 B \\
\hline Created: 29-September-2000 & IBIS Project & Revision B \\
\hline Revised: 13-January-2003 & Preliminary Design Study & Page 156 of 156 \\
\hline
\end{tabular}

108. Young, B. M., et al.

NUCS: A Nonuniformity Correction Algorithm for SGS/IQ3 Gamma-Ray Scanner, Proceedings of the $6^{\text {th }}$ Annual Nondestructive Assay Waste Characterization Conference, Salt Lake City, Utah, 1998, pp 317.

109. Zakharchenko, V. F.

"The Effect of Transport on the Time Distribution of Slowed Neutrons and on Pulsed Neutron Logs," Bulletin of Academy of Science USSR, Geophysics Series, Number 6, pp 845-851, 1962. 